\title{
Technical Debt Management in the Context of Agile Methods in Software Development
}

\author{
Graziela Simone Tonin
}

\author{
THESIS PRESENTED \\ AT \\ INSTITUTE OF MATHEMATICS AND STATISTIC \\ OF THE \\ UNIVERSITY OF SÃO PAULO \\ $\mathrm{TO}$ \\ OBTAIN THE TITLE \\ $\mathrm{OF}$ \\ DOCTOR IN SCIENCE
}

\author{
Program: Computer Science \\ Advisor: Prof. Dr. Alfredo Goldman \\ Co-advisor: Prof. Ph.D. Carolyn Seaman
}

This research was supported by Fapesp - Brazil proc.2011/23470-7, CNPQ - Brazil proc. 555595/2010-2, and IBM proc.207731/2014-4

São Paulo, March, 2018 


\section{Technical Debt Management in the Context of Agile Methods in Software Development}

The original version of the Ph.D. thesis in Computer Science presented by the candidate Graziela Simone Tonin to a judging committee. 


\section{Technical Debt Management in the Context of Agile Methods in Software Development}

The original version of the Ph.D. thesis in Computer Science presented by the candidate Graziela Simone Tonin to a judging committee.

Doctoral Thesis Committee:

- Prof. Ph.D. Carolyn Seaman (Co-Advisor) - (UMBC - Department of Information Systems) - USP - University of São Paulo

- Prof. Ph.D. Damian Andrew Tamburri - POLIMI - Department of Electronics, Information, and Bioengineering

- Prof. Dr. Eduardo Guerra - INPE - Associated Laboratory for Computing and Applied Mathematics

- Prof. Dr. Guilherme Horta Travassos - UFRJ - Systems Engineering and Computer Science Program

- Prof. Ph.D. Xiaofeng Wang - UNIBZ - Faculty of Computer Science 


\section{Abstract}

TONIN, G. S. Technical Debt Management in the Context of Agile Methods in Software Development. Thesis (PhD) - Institute of Mathematics and Statistics, University São Paulo, São Paulo, 2018.

The technical debt field covers an critical problem of software engineering, and this is one of the reasons why this field has received significant attention in recent years. The technical debt metaphor helps developers to think about, and to monitor software quality. The metaphor refers to flaws in software (usually caused by shortcuts to save time) that may affect future maintenance and evolution. It was created by Cunningham to improve the quality of software delivery. Many times the technical debt items are unknown, unmonitored and therefore not managed, thus resulting in high maintenance costs throughout the software life-cycle.

We conducted an empirical study in an academic environment, during two offerings of a laboratory course on Extreme Programming (XP Lab) at University of São Paulo and in two Brazilian Software Companies (Company A and B). We analyzed thirteen teams, nine in the Academy and four in the Companies environment. The teams had a comprehensive lecture about technical debt and several ways to identify and manage technical debt were presented. We monitored the teams, performed interviews, did close observations and collected feedback.

The obtained results show that the awareness of technical debt influences team behavior. Team members report thinking and discussing more on software quality after becoming aware of technical debt in their projects. We identified some impacts on the teams and the projects after having considered technical debt. A conceptual model for technical debt management was created including ways of how identifying, monitoring, categorizing, measuring, prioritizing, and paying off the technical debt. A few approaches and techniques for the technical debt management, identification, monitoring, measure, and payment are also suggested.

Keywords: Technical Debt, Technical Debt Management, Technical Debt Awareness, Identifying Technical Debt, Monitoring Technical Debt, Technical Debt Impact, Software Quality. 


\section{Resumo}

TONIN, G. S. Gerenciamento de Dívida Técnica no Contexto de Desenvolvimento de Software Ágil. Thesis (Doctorate Degree) - Instituto de Matemática e Estatística, Universidade de São Paulo, São Paulo, 2018.

A metáfora de dívida técnica engloba um importante problema da engenharia de software e essa é uma das razões pelas quais este campo tem recebido uma grande atenção nos últimos anos. Essa metáfora auxilia os desenvolvedores de software a refletirem sobre e a monitorarem a qualidade de software. A metáfora se refere a falhas no software (geralmente causadas por atalhos para economizar tempo) que podem afetar a futura manutenção e evolução do mesmo. A metáfora foi criada por Cunningham com o objetivo de melhorar a qualidade das entregas de software. Muitas vezes as dívidas técnicas não são conhecidas, monitoradas e nem geridas, resultando em um alto custo de manutenção ao longo do ciclo de vida do software.

Logo, conduziu-se um estudo empírico na academia, durante duas ofertas da disciplina de Programação Extrema (XP Lab) na Universidade de São Paulo e em duas empresas Brasileiras de desenvolvimento de software (Empresa A e B). Foram analisados treze times, sendo nove na academia e quatro nas empresas. Os times tiveram uma apresentação sobre dívida técnica e foram apresentadas algumas sugestões de abordagens para gerir dívida técnica. Monitorou-se os times, foram realizadas entrevistas, observações fechadas e informações foram coletadas.

Os resultados mostraram que considerar dívida técnica influenciou o comportamento dos times. Eles reportaram que após considerar dívida técnica passaram a refletir e discutir mais a qualidade do software. Identificou-se alguns impactos nos times e nos projetos depois de considerarem dívida técnica. Um modelo conceitual para gestão de dívida técnica foi criado, incluindo formas, técnicas e abordagens de como identificar, monitorar, categorizar, medir, priorizar e pagar os itens de dívida técnica.

Palavras-chave: Dívida Técnica, Gestão de Dívida Técnica, Consciência de Dívida Técnica, Identificação de Dívida Técnica, Monitoramento de Dívida Técnica, Impacto de Dívida Técnica, Qualidade de Software. 


\section{Acknowledgments}

My sincere gratitude to everyone who, in any way, has been part of my life at this important moment. People who have supported and helped me along the way.

I would like to thank my advisor, Professor Alfredo Goldman, for his relentless support in all these years. For encouraging me and opening doors so that I could achieve my objective. Professor Alfredo, who is a brilliant scientist, has taught me so much more than science and technical debt, he has taught me what true advisor is like. He did everything in his power to help me win awards, like the IBM Fellowship, attend renowned scientific summits and never stop learning. Beyond it all, he was always concerned about my study-life balance. Professor, thank you so much for your support, tips, suggestions, advice, and proofreading. I am forever thankful to you.

I would also like to express my gratitude to my co-advisor, Professor Carolyn Seaman, for the unconditional support troughtout my $\mathrm{PhD}$, especially when we worked together at UMBC. She made me feel warm and at home even in negative temperatures, and despite being so far from home. I am grateful to you and your amazing family. Working with you every day is having the opportunity to be inspired, evolve and learn, while standing next to a brilliant mind and a big heart. Meeting you was one of the best aspects of my $\mathrm{PhD}$ and what an honor it was to work with you. Thank you so much for your support, tips, advice, suggestions, and proofreading. I will be forever grateful to you for teaching me and inspiring with so much dedication and love.

Dear advisor and co-advisor, you are not just excellent advisers, you inspire me. It is a privilege to have had you on my journey.

A big thanks to the Professors who accepted to contribute to this research as a member of the Thesis committee. Thank you for sharing your knowledge and experience at this very important moment in my life. Thank you so much, Professors PhD. Damian Andrew Tamburri, Dr. Eduardo Guerra, Dr. Guilherme Horta Travassos and PhD. Xiaofeng Wang. I truly admire your work.

A big thanks to the Federal University of Fronteira Sul and to the magnificent Dean Professor Jaime Giolo for authorizing my absence while I was working on my thesis. The heads of departments Rosane Binotto and Lísia Regina Ferreira for approving and encouraging the training and learning of our professors. A big thanks to the current and former coordinators of the Computer Science course for always collaborating to make it possible and providing a compatible schedule, and to all the colleagues who supported me, encouraged and worked harder and longer so that I could focus on my thesis. A special thank you to Professor Dr. Raquel Pegoraro, who gave me all support since I started to UFFS.

A big thanks to the colleagues and friends I made at USP, who gave me support, working hard, sharing their knowledge and being crucial partners on this journey.

A special thanks to the English Teacher and Proofreader, who gave me such essential support, working hard, so that I could delivery my best. My affection and gratitude. 
A special thanks to my parents, my true heroes, who, by example, taught me to never give up on my dreams, even at difficult times. Despite not having the opportunity to study, they always encouraged and supported me unconditionally in the pursuit of my dreams and never let me give up the knowledge. Today, if I have the opportunity to achieve the highest level of knowledge it is because of the many years you have worked hard on the farm so that I could have the privilege of dedicating to my studies. This achievement is a tribute to you. I love you! To my sisters who have always supported me and have been worried about my well-being to endure the hardships of life. Thank you so much! I love you both! To my brother, sister-in-law and the little nephew and niece who even though live so far away but are always rooting for my success. My little niece, this brilliant mind that inspires and motivates me to go beyond. Thank you all. Family, I love you all and without your support, none of this would have been possible.

My aunt Nair, uncle Jacir, godfathers Glaucia and Ivan, cousins Enriete, Everton and Tuilo, and all their lovely family who take care of me in important things in life that I often can not care. Thank you for being my daily support and making me strong to move forward. You were instrumental in making this achievement possible. My gratitude! I love you!

To my grandparents, those who support me and protect me from the skies, and especially to my grandmother Alfreda, and the grandfather Alcides, who in his 93 years gives us the privilege of learning so much by sharing his wisdom. GrandFa what a great example you are. I want you to know I always follow your advice and teachings - and as you say, "You know". I love you grandfather.

A big thank you to my cousins, uncles, aunts, for supporting me and making my days lighter. My gratitude. I love you!

To my friends, who sent me many messages, who support me, forgive my absence, and give me the most invigorating hugs: thank you, thank you and thank you! To those who are close and those who live far, thank you for your unconditional love and support, you are my example. You are the lightness, joy and strength that allow me to rise and move on. I love you!

A very special thank you to the love of my life, Michel Miotto Barbosa, this brilliant mind that inspires me. You are so genial and your heart is so human. You are incredible, is my mentor and guide, a true idol. There are no words to describe how fantastic you have been on this long journey. Michel always absolutely and unconditionally supports and encourages my dreams, either by sponsoring them or by turning the night by my side just to ensure I am well.Your support makes a world of difference. Michel is always worried - often more than myself - if I am well fed, if I am warm in the American winter, if I am fine, whether I need a 'mate', a coffee or a hug. Thank you for understanding and enduring my absence, the stressful days and for always being there. No matter where I am following my dreams, I know that somewhere in the world I have there is an incredible man that I can count on unconditionally. How could I not fall in love with you every day?! This achievement not only is dedicated to you, it has your fundamental participation, this is your achievement too, my love. You are the best! Thank you! I love you!

In particular, I dedicate this thesis to the memory of two dear friends, Tania Mara Ferla and Daniel Dalabona, I miss you guys. Two incredible humans now shining from the sky.

A special thank you to those who spend some time sharing their knowledge and discussing the models and results of this research, especially to Joseph Yoder, a bright mind.

To you all!!! My affection and gratitude! 


\section{Contents}

\begin{tabular}{ll}
\hline Acknowledgments & iii
\end{tabular}

\begin{tabular}{ll}
\hline List of Abbreviations & ix
\end{tabular}

\begin{tabular}{|l|l}
\hline List of Figures & $\mathrm{x}$ \\
\hline
\end{tabular}

List of Tables $\quad$ xiii

$\begin{array}{llr}1 & \text { Introduction } & 1\end{array}$

1.1 Research Context . . . . . . . . . . . . . . . . . . . . . . . . . 3

1.2 Research Questions . . . . . . . . . . . . . . . . . . . . . . . . 4

1.3 Research Design $\ldots \ldots \ldots \ldots \ldots \ldots$

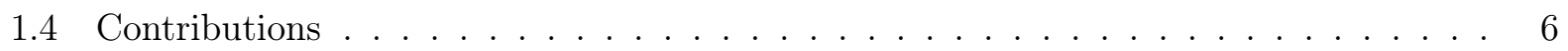

1.5 Thesis Structure $\ldots \ldots \ldots \ldots$

\begin{tabular}{|lll}
2 & Technical Debt & $\mathbf{8}$
\end{tabular}

2.1 The Technical Debt Metaphor . . . . . . . . . . . . . . . . . . . . . . . . 9

2.2 Technical Debt Characteristics $\ldots \ldots \ldots \ldots \ldots \ldots \ldots$

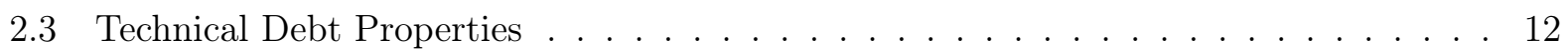

2.4 Incurring Technical Debt . . . . . . . . . . . . . . . . . . . . . . . . . . . . . . . . . . .

2.5 Impact of Technical Debt $\ldots \ldots \ldots \ldots \ldots \ldots \ldots \ldots$

2.6 Technical Debt Management $\ldots \ldots \ldots \ldots \ldots \ldots$

2.6 .1 What Is Not Technical Debt . . . . . . . . . . . . . . . . . . 27

2.6 .2 Technical Debt Prevention . . . . . . . . . . . . . . . . . . . . . . . . . 29

2.6 .3 Technical Debt Identification $\ldots \ldots \ldots \ldots \ldots$

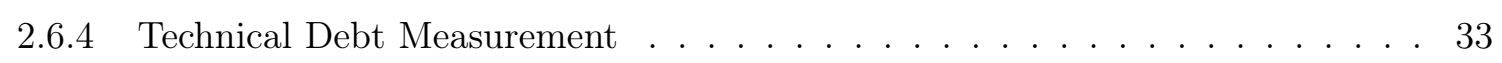

2.6 .5 Technical Debt Monitoring $\ldots \ldots \ldots \ldots \ldots . \ldots \ldots$

2.6 .6 Technical Debt Communication $\ldots \ldots \ldots \ldots \ldots \ldots$

2.6 .7 Tools to Support Technical Debt Management . . . . . . . . . . . . . . . 36

2.6 .8 Technical Debt Prioritization and Decision Making . . . . . . . . . . . . . . . . . . 37 2.6 .8 .1 Technical Debt Payment . . . . . . . . . . . . . . . 38

$2.7 \quad$ Chapter Summary $\ldots \ldots \ldots \ldots \ldots$

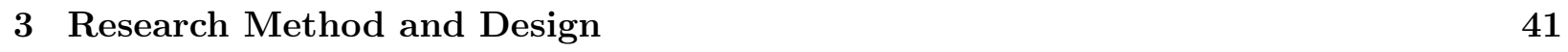

3.1 Research Method and Design . . . . . . . . . . . . . . . . . . . 41

$3.1 .1 \quad$ Case Study $\ldots \ldots \ldots \ldots \ldots$. . . . . . . . . . . . . . . . . . . . . . . . . . . . . . 41 
3.1 .2 Action Research $\ldots \ldots \ldots \ldots$. . . . . . . . . . . . . . . . 42

3.2 Methods and Techniques of Data Collection and Analysis _ . . . . . . . . . . . . 43

3.2 .1 Method of Data Analysis . . . . . . . . . . . . . . . . . 44

3.3 Research Steps Overview $\ldots \ldots \ldots \ldots$. . . . . . . . . . . . . . . . . . . . . . . . . . . . . . . . .

3.4 Chapter Summary $\ldots \ldots \ldots \ldots$. . . . . . . . . . . . . . . . . . . . . . . . . . . 48

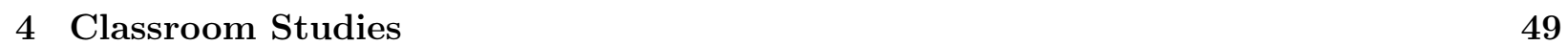

4.1 The Extreme Programming Course $\ldots \ldots \ldots$. . . . . . . . . . . . . . . . . . . 49

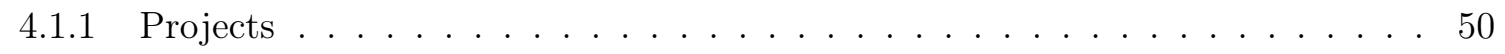

4.1 .2 The Informative Workspace $\ldots \ldots \ldots \ldots$. . . . . . . . . 50

4.2 Research Design $\ldots \ldots \ldots \ldots$. . . . . . . . . . . . . . . . . . . . . . . . . 53

4.2 .1 Technical Debt Identification $\ldots \ldots \ldots \ldots \ldots$

$4.2 .1 .1 \quad$ Extreme Programming Laboratory 2013 Offering . . . . . . . . . . . 53

$4.2 .1 .2 \quad$ Extreme Programming Laboratory 2014 Offering . . . . . . . . . . . 54

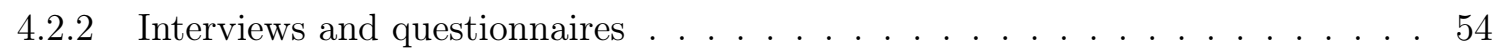

$4.2 .2 .1 \quad$ Extreme Programming Laboratory 2013 Offering . . . . . . . . . . . 54

$4.2 .2 .2 \quad$ Extreme Programming Laboratory 2014 Offering . . . . . . . . . . . 55

4.2 .2 .3 Data Analysis . . . . . . . . . . . . . . . 56

4.3 Results . . . . . . . . . . . . . . . . . . . . . . . . . . 56

$4.3 .1 \quad$ Extreme Programming Laboratory 2013 Offering $\ldots \ldots \ldots$. . . . . . . . 56

$4.3 .2 \quad$ Extreme Programming Laboratory 2014 Offering $\ldots \ldots \ldots \ldots$. . . . . . . . . 58

4.3 .3 Open Coding . . . . . . . . . . . . . . . . . . . . . 60

4.3 .4 Axial Coding . . . . . . . . . . . . . . . . . . . . 60

4.3 .5 Selective Coding . . . . . . . . . . . . . . . . . . . 62

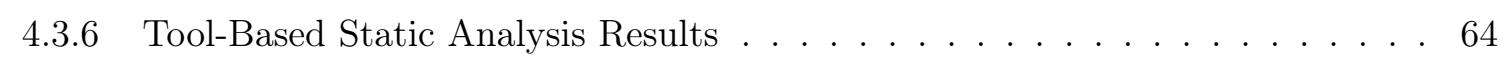

4.3 .7 Threats to the Validity $\ldots \ldots \ldots \ldots \ldots$

4.4 Chapter Summary $\ldots \ldots \ldots \ldots$

\begin{tabular}{|lll}
5 & Company A Studies & $\mathbf{6 8}$
\end{tabular}

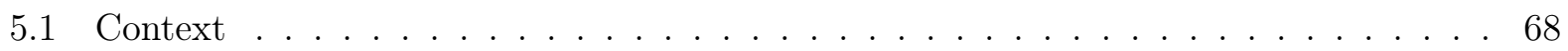

$5.1 .1 \quad$ Study $01 \ldots \ldots \ldots \ldots \ldots$

5.1 .1 .1 Research Design . . . . . . . . . . . . . . . . 70

5.1 .1 .2 Results . . . . . . . . . . . . . . . . . . . 71

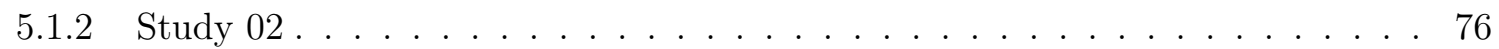

5.1 .2 .1 Research Design . . . . . . . . . . . . . . . 77

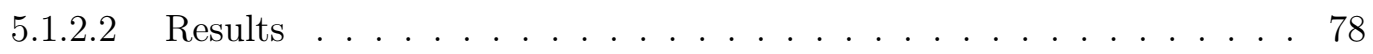

5.1.2.2.1 The Results of Data Analysis with Grounded Theory . . . 78

5.1 .2 .2 .1 .1 Open Coding . . . . . . . . . . . . 78

$5.1 .2 .2 .1 .2 \quad$ Axial Coding . . . . . . . . . . . . 78

$5.1 .2 .2 .1 .3 \quad$ Selective Coding . . . . . . . . . . . 80

5.1 .2 .2 .2 The Proposed Template . . . . . . . . . . . . . . . 80

5.1.2.2.3 The Conceptual Model for Technical Debt Management . . 82

5.1 .3 Threats to the Validity . . . . . . . . . . . . . . . 84

5.2 Chapter Summary $\ldots \ldots \ldots \ldots$. . . . . . . . . . . . . . . . . . . . . 84 
\begin{tabular}{llr}
\hline 6 & Company B Studies & 85
\end{tabular}

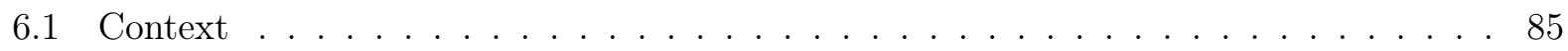

6.1 .1 Team A . . . . . . . . . . . . . . . . . . . . 86

6.1 .2 Team B . . . . . . . . . . . . . . . . . . . 86

6.1 .3 Research Design . . . . . . . . . . . . . . . . . . 86

6.1 .3 .1 Team A . . . . . . . . . . . . . . . . . . 86

$6.1 .3 .1 .1 \quad$ First Cycle $\ldots \ldots \ldots \ldots$. . . . . . . . . . . . 87

6.1 .3 .1 .2 Second Cycle $\ldots \ldots \ldots$. . . . . . . . . . . . 88

6.1 .3 .1 .3 Third Cycle. . . . . . . . . . . . . . . . . . . . . 89

6.1 .3 .2 Team B . . . . . . . . . . . . . . . . . . . . . 91

$6.1 .3 .2 .1 \quad$ First Cycle $\ldots \ldots \ldots \ldots$. . . . . . . . . . . . 91

6.1 .3 .2 .2 Second Cycle $\ldots \ldots \ldots$. . . . . . . . . . . . . 91

6.1 .3 .2 .3 Third Cycle $\ldots \ldots \ldots$. . . . . . . . . . . . . . . . . 93

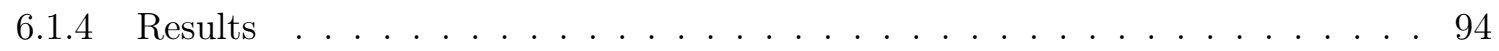

6.2 Chapter Summary $\ldots \ldots \ldots \ldots$

$\begin{array}{lll}7 & \text { Conceptual Model for Technical Debt Management } & 97\end{array}$

7.1 The Model . . . . . . . . . . . . . . . . . . . . . . . . . . . . . 97

7.1 .1 Technical Debt Identification $\ldots \ldots \ldots \ldots$. . . . . . . . . . . . . 98

7.1 .2 Technical Debt Management ．.. . . . . . . . . . . . . . . . . . . 101

$7.1 .2 .1 \quad$ Comparison with the Models . . . . . . . . . . . . . . . . 107

$7.1 .3 \quad$ Framework for Technical Debt Management . . . . . . . . . . . . . . . . . 107

$7.1 .4 \quad$ Framework to Manage and Reduce Technical Debt . . . . . . . . . . . . . . 108

7.1 .5 Conceptual Model of Technical Debt . . . . . . . . . . . . . . . . . . . 109

7.1 .6 Technical Debt Management Framework . . . . . . . . . . . . . . . . . . . 110

7.1 .7 Managing Architectural Technical Debt: A Unified Model . . . . . . . . . . . 112

7.1 .8 Technical Debt Management Cycle . . . . . . . . . . . . . . . . . . . . . . 113

7.1 .9 Summary of the Comparison of the Models $\ldots \ldots$. . . . . . . . . . . 114

7.1 .9 .1 Threats to the Validity . . . . . . . . . . . . 116

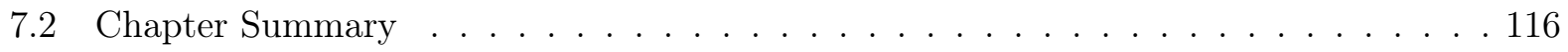

8 Conclusion 118

$8.1 \quad$ Summary of the Findings $\ldots \ldots \ldots \ldots \ldots$

8.2 Discussion of findings by each research question. . . . . . . . . . . . . . . . . 120

8.2.1 RQ1 (Company A): Which decisions may motivate a team to incur technical debt in agile software development projects? . . . . . . . . . . . . . . . . 121

8.2 .2 RQ2 \& RQ6 (Company A and B): What types of technical debt items did the agile software development team incur? . . . . . . . . . . . . . . . . . 121

8.2 .3 RQ3 (Company A): What is the impact on an agile software development team of the technical debt in their project? . . . . . . . . . . . . 121

8.2.4 RQ4 \& RQ7 (Company A and B): Which techniques and tools can agile software development teams use to identify, monitor and manage technical

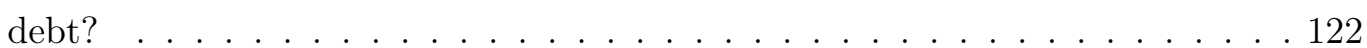


8.2.5 RQ5 \& RQ8 (Company A and B): What is the impact on the software development process when the agile software development team considers technical debt items? . . . . . . . . . . . . . . . . . . . . . . . . . . . . . . . . . . 122

8.2.6 RQ9 (XP Lab): What is the impact on the team when technical debt is explicitly considered?. . . . . . . . . . . . . . . . . . . . . . . . . . . . . . . . 122

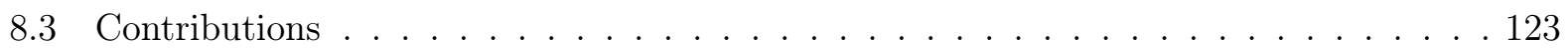

8.4 Future Works $\ldots \ldots \ldots \ldots$. . . . . . . . . . . . . . . . . . . . . . . . . 123

8.5 Chapter Summary $\ldots \ldots \ldots$. . . . . . . . . . . . . . . . . . . . . 124

\begin{tabular}{|lr}
\hline A Paper published at XP Conference, 2017 & 125
\end{tabular}

B Paper published at Brazilian Workshop of Agile Methods, 2014 142

\begin{tabular}{|ll|}
\hline C Short Paper published at Agile Conference, 2014 & 155
\end{tabular}

D Paper published at Brazilian Workshop of Agile Methods, 2012. 158

\begin{tabular}{|lll}
\hline E & First Interview applied in Extreme Programming Laboratory, 2013 Edition & 171
\end{tabular}

F $\quad$ First Questionnaire Applied in Extreme Programming Laboratory, 2014 Edition 74

G Second Questionnaire Applied in Extreme Programming Laboratory, 2014 Edi-

tion

H Questionnaire applied in the Company A 


\title{
List of Abbreviations
}

\author{
XP (eXtreme Programming) \\ XP Lab eXtreme Programming Laboratory \\ TD Technical Debt \\ IT Information Technology \\ PO Product Owner
}




\section{List of Figures}

1.1 Cost of Change . . . . . . . . . . . . . . . . . . . . . . . . 2

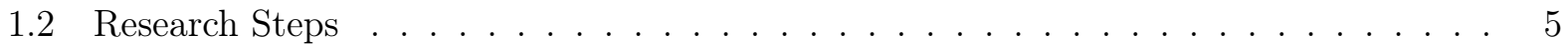

2.1 Technical Debt Search $\ldots \ldots \ldots \ldots \ldots$

2.2 Technical Debt Landscape $\quad \ldots \ldots \ldots \ldots$

2.3 Technical Debt Quadrant $\ldots \ldots \ldots \ldots \ldots$. . . . . . . . . . . . . . 11

2.4 Technical Conceptual Model $\ldots \ldots \ldots \ldots$. . . . . . . . . . . . . . . 12

2.5 Technical Debt Properties $\ldots \ldots \ldots \ldots \ldots \ldots$. . . . . . . . . . . . . . . 14

2.6 Technical Debt Cost $\ldots \ldots \ldots \ldots \ldots \ldots \ldots \ldots$

2.7 Technical Debt Management Cycle ～. . . . . . . . . . . . . . . . . . . . 20

2.8 Four Quadrant Model $\ldots \ldots \ldots \ldots$. . . . . . . . . . . . . . . . . . . . . . . . . 21

2.9 Framework for Technical Debt Management. . . . . . . . . . . . . . . . . . . . . 21

2.10 Activities for Technical Debt Management. [79]. . . . . . . . . . . . . . . . . . 22

2.11 Technical Debt Management Framework . . . . . . . . . . . . . . . . . . . . . . 23

2.12 Framework for the Elements for Technical Debt Management . . . . . . . . . . . . . 24

2.13 Framework to Manage and Reduce Technical Debt . . . . . . . . . . . . . . . . . . . 24

2.14 Technical Debt Management Framework . . . . . . . . . . . . . . . . . . . . 26

2.15 Technical Debt Management Framework . . . . . . . . . . . . . . . . . . . 27

2.16 Non-technical debt . . . . . . . . . . . . . . . . . . . . . . . . . . . . 28

2.17 Technical Debt Identification Decision Tree $\ldots \ldots \ldots \ldots$. . . . . . . . . . . . . 29

2.18 Examples of Technical Debt . . . . . . . . . . . . . . . . . . . . . 32

2.19 Cost of Technical Debt by Type . . . . . . . . . . . . . . . . . . . . . . 34

2.20 Ways to make the technical debt visible . . . . . . . . . . . . . . . . . 36

2.21 List of Technical Debt Priorities . . . . . . . . . . . . . . . . . . . . . . . . 38

2.22 The organization of "right code" requirements in a chronological order. . . . . . . . . 39

3.1 Action Research Cycle . . . . . . . . . . . . . . . . . . . . . . . . . . . . . 43

3.2 Research Timeline . . . . . . . . . . . . . . . . . . . . . . . . . . . . . . . 46

3.3 Research Steps $\ldots \ldots \ldots \ldots \ldots \ldots$. . . . . . . . . . . . . . . . . . . . . . 47

4.1 Technical Debt Boards $\ldots \ldots \ldots \ldots \ldots \ldots$. . . . . . . . . . . . . . . 51

4.2 Card with the Information about the Technical Debt Item. . . . . . . . . . . . . 51

4.3 Technical Debt Item . . . . . . . . . . . . . . . . . . . . . . . . . . . 52

4.4 Steps of the XP Lab 2013 Offering $\ldots \ldots \ldots \ldots \ldots$

4.5 Steps of the XP Lab 2014 Offering $\ldots \ldots \ldots \ldots \ldots \ldots$ 
4.6 Technical Debt Concept $\ldots \ldots \ldots \ldots \ldots \ldots$

4.7 Percentage of People Who Considered Technical Debt in their Projects. . . . . . . . 57

4.8 Categories and Subcategories of the XP Lab 2013 and 2014 Offering . . . . . . . . . 61

4.9 XP Lab 2013 Edition $\ldots \ldots \ldots \ldots \ldots$

4.10 XP Lab 2014 Edition . . . . . . . . . . . . . . . . . . . . . . . . 65

5.1 Moment in which the Major Changes Occurred in the Cases Investigated. . . . . . . 70

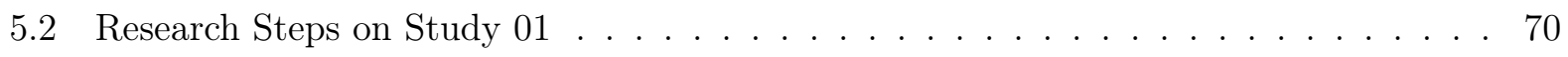

5.3 Steps to Identify Big Changes . . . . . . . . . . . . . . . . . . . . . . . . 71

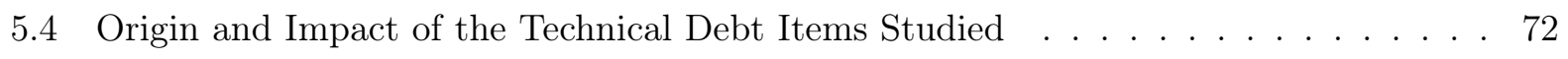

5.5 Effort Spent Due to the Incurrence of Technical Debt Items Per Project . . . . . . . 73

$5.6 \quad$ Case 01 - Upgrade of the jQuery Version . . . . . . . . . . . . . . . 73

5.7 Case 02 - Definition of The Persistence Form of Monitoring Events . . . . . . . . . . 74

5.8 Case 03 - Using MON as a Monitoring Agent in the Tool . . . . . . . . . . . . . . . 74

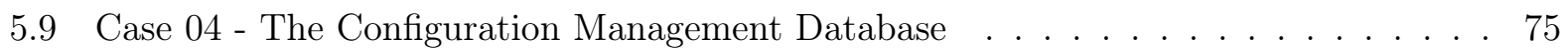

5.10 Technical Debt Categorization . . . . . . . . . . . . . . . . . 76

5.11 Research Steps on Study $02 . \quad \ldots \ldots \ldots$. . . . . . . . . . . . 77

5.12 Categories resulting from the open coding. . . . . . . . . . . . . . . . . . . 79

5.13 Categories Resulting from the Evaluation of the Conceptual Model for Technical

Debt Management . . . . . . . . . . . . . . . . . . . . 79

5.14 Relationships between Categories and Codes . . . . . . . . . . . . . . . . 80

5.15 Type of Story Technical Debt . . . . . . . . . . . . . . . . . . . 81

5.16 Type of Technical Debt Items $\ldots \ldots \ldots 2 \ldots$

5.17 Technical Debt Item . . . . . . . . . . . . . . . . . . . . 82

5.18 Board of Tasks with Technical Debt Items $\ldots \ldots \ldots$. . . . . . . . . . . 83

5.19 Main Results of Company A . . . . . . . . . . . . . . . . . 83

$6.1 \quad$ Action Research Cycles by Team. . . . . . . . . . . . . . . . . . . . . . 87

$6.2 \quad$ Graph Generated by the Pull Requests Analysis Tool . . . . . . . . . . . . . . . . . 89

6.3 Coverage of Unit Tests $\ldots \ldots \ldots \ldots \ldots \ldots \ldots \ldots$

6.4 Technical Debt Items Incurred Per Day $\ldots \ldots \ldots$. . . . . . . . . . . . . . . . 90

6.5 Board with the Technical Debt Items of Team B in Company B . . . . . . . . . . . . 92

6.6 Fields that should be Completed in the Technical Debt Form. . . . . . . . . . . . . 92

6.7 Technical Debt Items on the Scrum Board. . . . . . . . . . . . . . . . . . 94

6.8 Technical Debt Item in the JIRA Tool. . . . . . . . . . . . . . . . . . . . . . . . 94

6.9 Technical Debt Items Incurred and Paid. . . . . . . . . . . . . . . . . . . . 95

6.10 Amount of Technical Debt Items Inserted in the Software Versus Code per Team. . . 95

$7.1 \quad$ Conceptual model for Technical Debt Management. . . . . . . . . . . . . . . . . . . 98

7.2 Conceptual Model for Technical Debt Management . . . . . . . . . . . . . . . . . . . 99

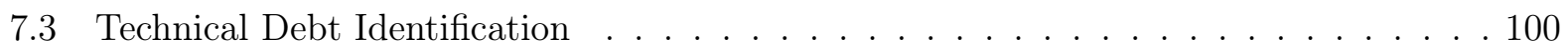

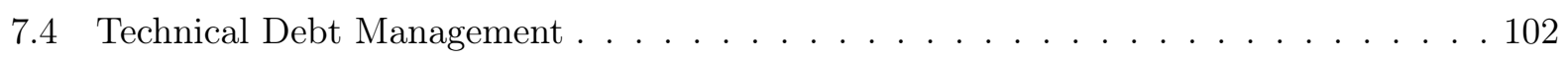

7.5 Conceptual Model for Technical Debt Management according to the Academic Stud-

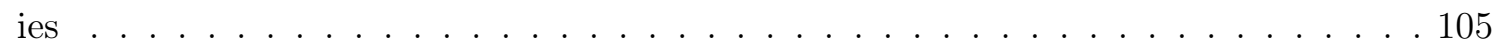


7.6 Conceptual Model for Technical Debt Management according to the Company A

Studies . . . . . . . . . . . . . . . . . . . 106

7.7 Framework to technical debt management. . . . . . . . . . . . . . . 107

7.8 Framework to Manage and Reduce Technical Debt . . . . . . . . . . . . . . . . 108

7.9 Conceptual Model of Technical Debt ～. . . . . . . . . . . . . . . . . . . . 110

7.10 Technical Debt Management Framework ． . . . . . . . . . . . . . . . . . 111

7.11 Technical Debt Management Framework . . . . . . . . . . . . . . . . . . . . . . . 113

7.12 Technical Debt Management Cycle ． . . . . . . . . . . . . . . . . . . . 113

7.13 Framework for the Elements for Technical Debt Management . . . . . . . . . . . . 114

8.1 Conceptual Model for Technical Debt Management . . . . . . . . . . . . . . . . . . . 119 


\section{List of Tables}

2.1 Technical Debt Identification Items [42]. . . . . . . . . . . . . . . . . . . 14

$4.1 \quad$ Extreme programming projects. . . . . . . . . . . . . . . . . 50

4.2 Extreme programming projects. . . . . . . . . . . . . . . 60 


\section{Chapter 1}

\section{Introduction}

Every day, decision-makers in software development companies face a situation in which they have to produce software with inadequate budget and time without decreasing quality. In these situations, team members' opinions are divided about where to invest such limited resources. On one hand, investments can be made in quality, e.g., by improving the quality properties of code, architecture and documentation, given that these are essential investments for the sustainability of the system. However, these investments usually do not generate benefits in the short term and are largely invisible to managers and customers. The other option is to invest, as quickly as possible, in the development of new features that are important to the final customers in order to maximize short-term gain. The rapid development of features, without a parallel investment in quality, leads to low-quality code, which impacts long-term system sustainability. Studies show that maintenance is the most expensive phase of the software lifecycle, responsible for up to $90 \%$ of the total cost and up to $60 \%$ of the total effort of a software system's life [56]. According to some estimates around 250 billion lines of code were under maintenance in 2000 91. In the US in 2002, the software maintenance market ranges from $\$ 1,250$ to $\$ 2,600,000$ in annual expenditures [99]. Devoting such huge resources to maintenance decreases the resources available to develop the increasingly sophisticated software needed to meet the market demands. Therefore, it is essential to find ways to make maintenance more cost-effective, not only because maintenance is so costly, but also because the demands of new development are so compelling. The work described in this thesis aims to investigate factors that can help in this matter.

In the OOPSLA conference in 1992 [20]), Cunningham creates the first definition of the metaphor:

"...Although immature code may work fine and be completely acceptable to the customer, excess quantities will make a program unmasterable, leading to extreme specialization of programmers and finally an inflexible product. Shipping first time code is like going into debt. A little debt speeds development so long as it is paid back promptly with a rewrite. Objects make the cost of this transaction tolerable. The danger occurs when the debt is not repaid. Every minute spent on not-quite-right code counts as interest on that debt. Entire engineering organizations can be brought to a stand-still under the debt load of an unconsolidated implementation, object-oriented or otherwise..." [20]

After that, Cunningham made a video [?], to clarify that he had created the metaphor to explain the refactoring that was being done at the company where he worked. Since other definitions have been created, but the definition that guided this research was the Cunningham [20].

The technical debt is an important problem in software engineering and this has received significant attention in recent years [83. Its importance and relevance are largely due to its connection with cost over time [55]. Technical debt is recognized as a critical problem in the software development industry [101]. A Gartner research report [37] estimated that the total technical debt value was $\$ 500$ billion in 2010 and about $\$ 1$ trillion in 2015. Also, Rubin [79] indicates that when technical debt increases, not only will more time be necessary to implement changes, but cost will also increase, as we can observe in Figure 1.1 . 
Figure 1.1: Cost of Change

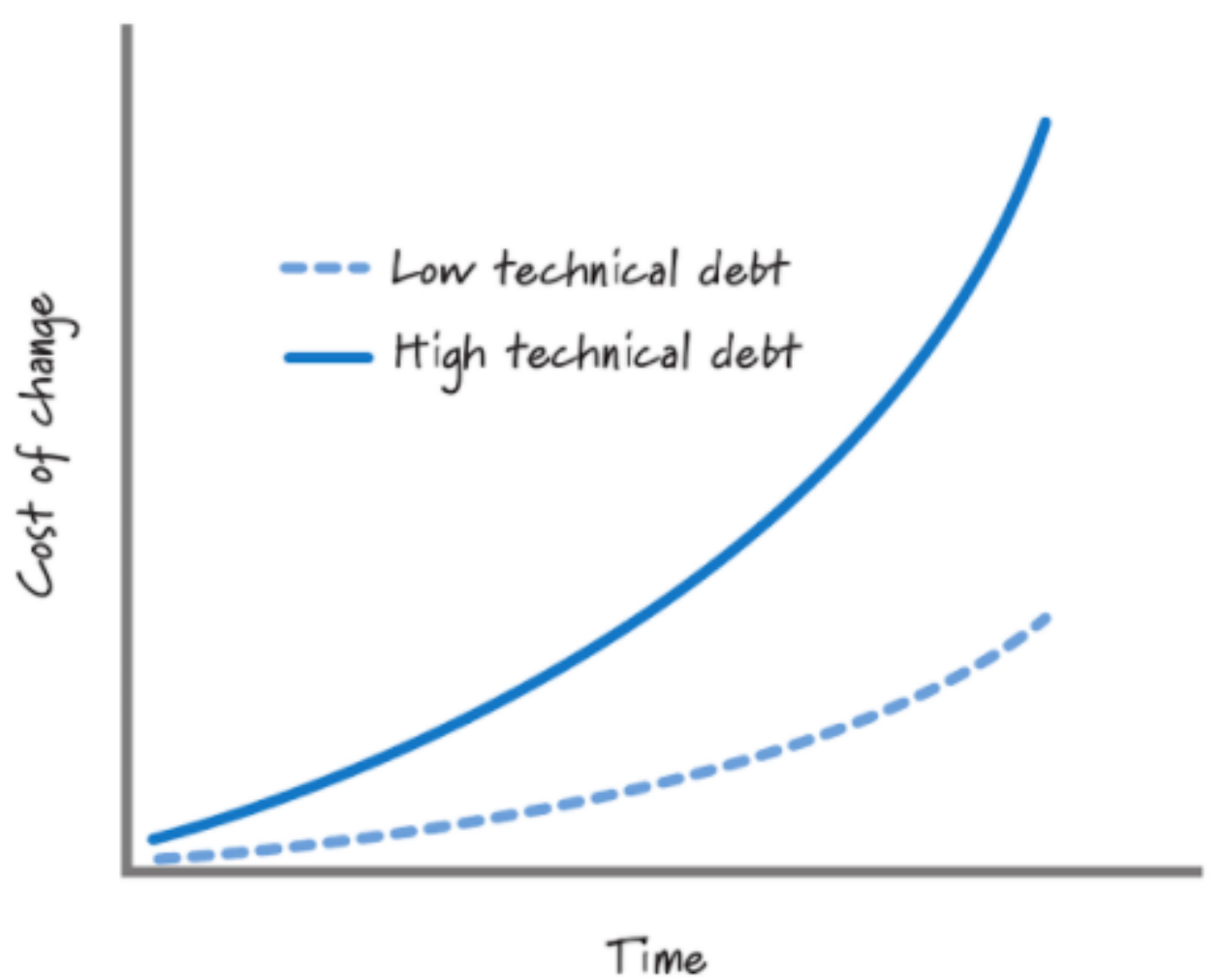

Source: [79].

For example, when technical debt is inserted due to a strategic decision, this could compromise the code quality as a consequence of the restrictions imposed on the process, such as time and resources. Also, developers often choose to compromise software quality to attend to other urgent demands in another dimension of the project, for instance, time-to-market. They incur a technical debt that needs to be paid in the future.

Technical debt [41] is a concept that makes an analogy with financial debt [55] [84]. In the analogy, when the technical debt is inserted it implies interest that may accrue in the form of extra time and effort when changes need to be made. These many technical debt items could be paid off through refactoring, or they could continue to incur interest. However, if there is no management, the technical debt could get out of control impacting the maintenance and evolution process due to the high effort that must be expended to pay them off eventually.

In contrast, the Agile Manifesto emphasizes that it is more important to adapt to changes throughout the software development process with continuous delivery $\left.\right|^{1}$ obtaining functional software. But often, there is an excessive preoccupation with continuous deliveries without due attention to quality and it may result in the incurrence of technical debt.

Because of the reasons related above, this research covers multiples case studies to explore whether and how technical debt is considered in agile team $2^{2}$. We aiming to identify the impact on the teams once they are aware of the technical debt, encourage attention to the concept, and suggest ways to identify, monitor and manage the technical debt. Finally, we formulate a conceptual model for technical debt management that can assist teams at the time of strategic decision-making.

\footnotetext{
$1 "$...Continuous Delivery is a software development discipline where you build software in such a way that the software can be released to production at any time..." 34

${ }^{2}$ We consider an agile team all the teams that work with agile methods and the teams that believe that they are agile.
} 


\subsection{Research Context}

In early 1967, an awareness of the importance and impact of software systems began to rise in the various activities of society. As a result of the many problems faced in software development, a general belief was created on what would be necessary to establish techniques/processes such as those already used in engineering to try to overcome the software crisis [62].

Later, engineers began to realize that the process model proposed in the Software Engineering Conference held in 1968, was very strict and inflexible to be followed in software development, where in many cases the requirements continue to change throughout the lifecycle of the project 62. So, in the late seventies, they started to consider other ways to improve this model. At that time, Lehman began to formulate the Laws of Software Evolution 62. These laws were created in the research made by Lehman to understand the process of change that was taking place in the IBM OS 360 and the results were confirmed in subsequent studies [53]. Lehman's laws, in general, describe features applicable to software development companies and dictates that some problems may be avoided during the software development cycle. Lehman also discovered that the software will always end up reaching a certain age when the decision of maintaining or discontinuing it is the result of the relationship between the cost of maintenance and the cost of developing new software [53].

When the technical debt metaphor emerged, one of its main goals was to help developers monitor the immature code of the software. These pieces of software may need to be corrected in the future due to the need of Software Evolution. The quality of the software is frequently compromised due to time restrictions, resources and knowledge imposed on the process.

Although research in this area has been growing, according to Zazworka et al. [112], this term has also been widely discussed in cyberspace, but often in a contradictory way. In this study, the authors developed a survey to analyze these discussions and identify issues of potential research interests in the area. Among the topics found are methods and techniques for managing and monitoring technical debt, team morale and motivation, and others. In another study Spinola et al. [93] investigated the technical debt folklore and as a result identified some areas that present research gaps in technical debt. Two important areas were directly connected with these studies. The first is to explore methods and techniques for tracking and managing technical debt. The second is the relationship between technical debt, team motivation and morale. Izurieta et al.[49] highlighted that some models summarizing the aspects connected to technical debt, but do not consider the activities for technical debt management. It should be noted that these studies highlighted gaps that directly link to the objectives of this study. Which has as main contribution the conceptual model for technical debt management.

According to Kruchten et al. [51], agile teams believe that they are immune to technical debt because they use an interactive development process. Therefore, he explains that in these teams, technical debt items could be incurred rapidly and massively, because often they develop and deliver very fast, with no time for good design or thinking in the long-term. It could result in incurring some technical debt such as a lack of rigor or systematic testing [51]. To deal with technical debt and avoid accumulating too much, Kruchten et al. [51] suggests: "The first step is awareness: identifying debt and its causes. The next step is to manage this debt explicitly, which involves listing debtrelated tasks in a common backlog during release and iteration planning, along with other "things to do". Nord et al. [67], also related that some agile approaches result in an implementation that accumulates debt 67. On the other hand, aiming to deliver functional software, focusing on what is important to the customer, the agile movement has made enormous progress in recent decades, significantly improving the software delivery and creating more satisfying work environments in organizations [45].

Therefore, this study aims to create a better understanding of the concept of technical debt in the context of agile teams and for this, we conducted some case studies. In particular, the case studies, performed both in an academic course and in agile companies, aim to determine whether technical debt has been considered in the process of software development. Based on these studies we have identified the technical debt impact and have created a conceptual model for technical debt 
management aiming to help teams consider technical debt in their projects.

\section{$1.2 \quad$ Research Questions}

The main objective of this research is to suggest an conceptua $\left.\right|^{3}$ model for technical debt management. Additionally, it explores the impact on agile teams once they were aware of the technical debt concept. The goal and questions that guided each study are described below.

In the Company A (fictitious name, for confidentiality issues), we did two studies. In the first study our objective was to investigate whether technical debt had been incurred. Also, we aimed at understanding the motivations and impact of the technical debt items. This study intends to answer the following questions:

- RQ1: Which decisions may motivate a team to incur technical debt in agile software development projects?

- RQ2: What types of technical debt items did the agile software development team incur?

- RQ3: What is the impact on an agile software development team of the technical debt in their project?

In the study at Company B (fictitious name, for confidentiality issues), our goal was to identify, monitor and manage technical debt items in an agile software development project team. The questions that guided this study were:

- RQ4: Which techniques and tools can agile software development teams use to identify, monitor and manage technical debt?

- RQ5: What is the impact on the software development process when the agile software development team considers technical debt items?

- RQ6: What types of technical debt items does the agile software development team?

In the second study, at Company A, our goal was to identify, monitor and manage technical debt items in an agile software development project team without interfering in the software development process. The questions that guided this study were:

- RQ7: Which techniques and tools can agile software development teams use to identify, monitor and manage technical debt?

- RQ8: What is the impact on the software development process when the agile software development team considers technical debt items?

Finally, in the fourth and fifth study at the Extreme Programming Laboratory course, 2013 and 2014 editions, the goal was to identify the impact on the agile software development team once they were aware of the technical debt concept. This study aimed at answering the following question:

- RQ9: What is the impact on the team when technical debt is explicitly considered?

\subsection{Research Design}

Five steps were developed aiming at finding answers to the research questions. In the table below we briefly describe each one. (This figure is described in detail in Chapter 3 .

In the first step, we did the literature review to identify studies about identification, monitoring, management, and impact of technical debt. We have also collected data related to forms, techniques, tools, and metrics for technical debt identification and monitoring.

\footnotetext{
${ }^{3}$ See more at https://plato.stanford.edu/entries/models-science/
} 
Figure 1.2: Research Steps

\begin{tabular}{|c|c|c|c|c|}
\hline & Study Name & Setting & $\begin{array}{c}\text { Research } \\
\text { Method }\end{array}$ & Main Objective \\
\hline Step 01 & $\begin{array}{l}\text { Literature } \\
\text { Review }\end{array}$ & Academic & $\begin{array}{l}\text { Literature } \\
\text { Review }\end{array}$ & $\begin{array}{l}\text { To identify studies on technical } \\
\text { debt }\end{array}$ \\
\hline \multirow{2}{*}{ Step 02} & $\begin{array}{l}\text { Company A - } \\
\text { First Study } \\
\end{array}$ & Company & Case Study & $\begin{array}{l}\text { To identify the impact of some } \\
\text { technical debt }\end{array}$ \\
\hline & Company B & Company & $\begin{array}{l}\text { Action } \\
\text { Research }\end{array}$ & $\begin{array}{l}\text { First Cycle - Start identifying } \\
\text { and monitoring technical debt }\end{array}$ \\
\hline \multirow{3}{*}{ Step 03} & Company B & Company & $\begin{array}{l}\text { Action } \\
\text { Research }\end{array}$ & $\begin{array}{l}\text { Second Cycle - To improve the } \\
\text { ways of identifying and } \\
\text { monitoring technical debt }\end{array}$ \\
\hline & $\begin{array}{l}\text { Company A - } \\
\text { Second Study }\end{array}$ & Company & Case Study & $\begin{array}{l}\text { Start identifying, monitoring } \\
\text { and management technical debt } \\
\text { without interfering in the } \\
\text { software development process } \\
\text { of the agile team }\end{array}$ \\
\hline & XP Lab 2013 & Academic & Case Study & $\begin{array}{l}\text { First analysis of the impact on } \\
\text { the team after considering } \\
\text { technical debt }\end{array}$ \\
\hline \multirow[b]{2}{*}{ Step 04} & Company B & Company & $\begin{array}{l}\text { Action } \\
\text { Research }\end{array}$ & $\begin{array}{l}\text { Third Cycle - Start to manage } \\
\text { technical debt }\end{array}$ \\
\hline & XP Lab 2014 & Academic & Case Study & $\begin{array}{l}\text { Second analysis of the impact } \\
\text { on the team after considering } \\
\text { technical debt }\end{array}$ \\
\hline \multirow{6}{*}{ Step 05} & PhD sandwich & Academic & - & Discussion of the data collected \\
\hline & Data Analysis & Academic & - & Analysis of the data \\
\hline & $\begin{array}{l}\text { Thesis Writing } \\
\text { in Portuguese }\end{array}$ & Academic & - & $\begin{array}{l}\text { Start writing the thesis in } \\
\text { Portuguese }\end{array}$ \\
\hline & $\begin{array}{l}\text { Thesis Writing } \\
\text { in English }\end{array}$ & Academic & - & $\begin{array}{l}\text { After the Dagstuh1 Seminar, } \\
\text { translate the thesis into English }\end{array}$ \\
\hline & Paper Writing & Academic & - & $\begin{array}{l}\text { Publish a paper at the XP } \\
\text { Conference }\end{array}$ \\
\hline & $\begin{array}{l}\text { Update the } \\
\text { Literature } \\
\text { Review }\end{array}$ & Academic & $\begin{array}{l}\text { Literature } \\
\text { Review }\end{array}$ & $\begin{array}{l}\text { Reading and analyzing recent } \\
\text { papers }\end{array}$ \\
\hline
\end{tabular}

In the second step, we carried out a case study with some software development teams in a Company A, a market leader in the technology sector. In this study, some technical debt impacts on the project were identified and analyzed in order to verify what was an impact on the project in a company that used the agile method Scrum. We sought to understand the importance of identifying technical debt. Furthermore, we intended to identify the reasons and motivations that resulted in incurring these technical debt items. In another software development company, Company B, we started the first action research cycle in order to identify and monitor technical debt. In this case, the goal was to use some techniques and forms found in the literature review to identify and monitor technical debt. The third step started once the agile software development teams in the Company A understood the relevance of managing technical debt. We decided to identify, monitor, and manage technical debt using the tools and the processes they had already used in software development. Then we created a preliminary model to help agile software development teams to manage technical debt. In addition, based on this preliminary model we started the second action research cycle in the Company $\mathrm{B}$, our focus was the identification, monitoring and management of technical debt. Moreover, we started an classroom study in an Extreme Programming Laboratory course, XP Lab 2013 (It is a course part of the Computer Science program of the Institute of Math- 
ematics, Statistics and Computer Science, at University of São Paulo) aimed at identifying whether considering technical debt, in fact, could result in some impact on an agile software development team.

In the fourth step, we did the third action research cycle in a Company $\mathrm{B}$, in order to create a model to identify, monitor and manage technical debt, based on the experience gained during the previous cycles. Furthermore, we replicated the classroom study at the Extreme Programming Laboratory course in the 2014 edition (XP Lab 2014).

Finally, in the fifth step, we focus on data analysis and refine the data analysis. Also, we did a Ph.D. sandwich at University of Maryland - Baltimore County, wrote the thesis, firstly in Portuguese and after in English. Moreover we update the literature review aimed to identify the recent studies in the technical debt management area. It is important to highlight that in the last years the studies in the area has considerably increased, so we focus on identify just the studies that were related to a conceptual model for technical debt management.

\subsection{Contributions}

This section presents the summary of the contributions of this research. As explained before, the main goal of this study is to suggest a model to identify, monitor, and manage technical debt as well as exploring its impact in agile teams once they are aware of the technical debt concept. Thus, the agile software development teams can use it to deal with technical debt in their projects. The main contributions of this research can be summarized as:

- We present the state of art in the technical debt management area and we did an analysis of several studies on technical debt identification, monitoring, payment, prevention, measuring, prioritization, etc. In Chapter 2 we have related several studies on technical debt management, identification, monitoring, payment, prevention. We also provide evidence of the empirical studies on technical debt in different agile development environments.

- A conceptual model for technical debt management. In this model we consider aspects of technical debt such as the reasons, motivations and the impact of technical debt on agile software development projects and teams. In addition, we show steps, approaches, techniques, and tools that the agile software development project teams could choose to identify, monitor, measure, prioritize and manage technical debt in their projects. Additionally, we did a comparative analysis of our conceptual model with similar models from the literature. Furthermore, the model with its current level of abstraction can be used as a guide for companies to define strategies for identification, monitoring, measurement and technical debt management.

- During our empirical studies, we always tried to let the developers use their current tools, adding whenever possible the technical debt awareness to their usual environment.

- The insights and results presented after the application of the model can help the teams to understand the challenges of dealing with technical debt.

\subsection{Thesis Structure}

In Chapter 2, the technical debt concept, properties and characteristics are presented. We also discuss incurring technical debt and technical debt impact. In addition, we relate to studies about technical debt management, including what is not technical debt. Furthermore, in that Chapter, we describe technical debt identification, prevention, measurement, prioritization, and monitoring. Chapter 3 explains the methods and steps of the research design. Chapter 4 presents what we did in the classroom study, the context, the research design, the results, and the discussion of the findings. Chapter 5 shows the details about the two studies we did in the first technology agile software company, including the context, the research design, the results, and the discussion of the 
findings. In Chapter 6 we explain the studied we conducted at the other agile software company, the context, the research design. Additionally, we present the final version of the conceptual model for technical debt management resulting from all the studies. Finally, in Chapter 8 we discuss the findings, comparing with the related work and answer the research questions. We also summarized the contributions and suggest future works. 


\section{Chapter 2}

\section{Technical Debt}

More than 25 years have passed since the technical debt metaphor was established in 1992 and since then the concept has become well known but not yet widely understood [36].

This metaphor is gaining strength and it is increasingly recognized as being of great use for technical communication and communication between engineers and executives [12, [84]. It can facilitate the discussion between technical and non-technical members of the team, also between customers and the teams [35, 93].

New academic studies are emerging [112], and the term has been widely discussed in cyberspace [55. In the last five years (2012-2017) the search volume for the term "technical debt" increased considerably, as we can see in Figure 2.1 .

Figure 2.1: Technical Debt Search

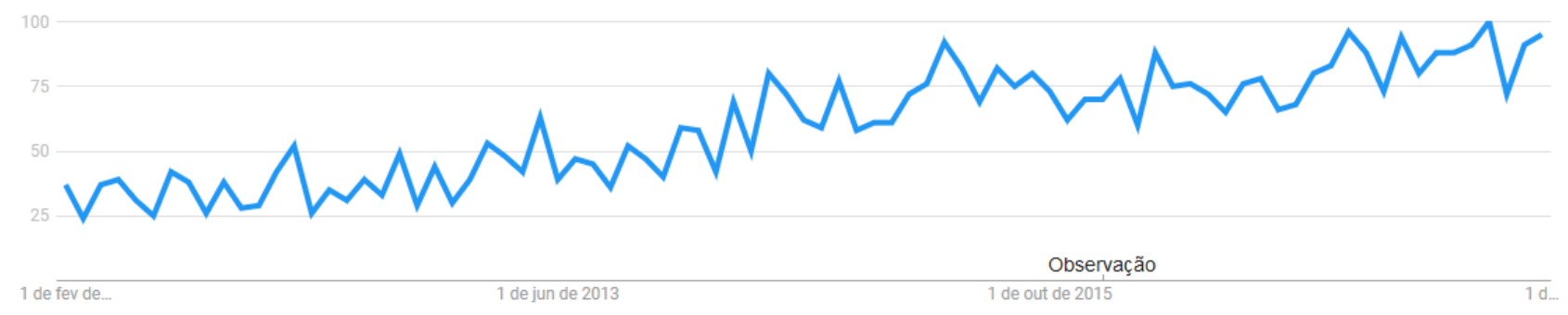

Source: Google Trends on Technical Debt

Despite all this growth and the discussions on the subject, the study of Zazworka [112] highlights that the term often was used in a contradictory way in cyberspace. Furthermore, in 2012 a study of Lim [57] pointed out that about $75 \%$ of professionals in the area were not familiar with the term.

In this chapter, we first give an overview of the technical debt metaphor and explain its properties and characteristics. We considered the studies reported in this chapter to explain the technical debt metaphor to the teams, and also they guided the discussions during the process of implementing technical debt management in the projects. We also used it to define which properties the teams could consider to identify the technical debt items. We discuss the implications of incurring technical debt and its impacts. Then, we give a detailed discussion of the current model on technical debt management and several works were used in this study. The first model on technical debt management developed by Seaman and Guo [87] guided our studies. Furthermore, throughout the chapter, we present the current works about technical debt management. In fact, when we started this study, we considered all the studies in technical debt management because there were not many studies on this subject. Since many studies appeared in the following years, we consider only studies directly connected with our study. We kept the studies previously used on different aspects of technical debt management. We also discussed some issues not strictly related to technical debt in order to 
pursue a more precise definition of technical debt and we give some examples of the different types of technical debt. In addition, we also discuss technical debt prevention. Additionally, we explain in more detail the related work on technical debt identification, monitoring and communication. Thus we relate some tools that could support technical debt management. Finally, we discuss approaches for technical debt prioritization and payment.

\subsection{The Technical Debt Metaphor}

Technical debt was first proposed as a metaphor by Cunningham [?]: one of its main goals was to help developers to monitor the immature code of their software. This is the main definition we followed during our research.

Cunningham in his report at the OOPSLA conference in 1992 [20]) presented the first definition of the metaphor:

"...Although immature code may work fine and be completely acceptable to the customer, excess quantities will make a program unmasterable, leading to extreme specialization of programmers and finally an inflexible product. Shipping first time code is like going into debt. A little debt speeds development so long as it is paid back promptly with a rewrite. Objects make the cost of this transaction tolerable. The danger occurs when the debt is not repaid. Every minute spent on not-quite-right code counts as interest on that debt. Entire engineering organizations can be brought to a stand-still under the debt load of an unconsolidated implementation, object-oriented or otherwise..." [20]

Although the technical debt metaphor was originally coined in reference to coding practices, its scope was later broadened to other parts of the software development lifecycle. In June 2010, in what is considered the first organized meeting of researchers in this area, the first Workshop on Managing Technical Debt was held at the Software Engineering Institute (SEI) in Pittsburgh, USA, with the objective to discuss technical debt and software management from a broader point of view. As a result, the metaphor has been extended to refer to any imperfect artifact in the software development lifecycle [12.

In addition to the definition created by Cunningham [20, other authors have created their versions to explain the meaning of the metaphor and to adapt it to different contexts.

According to Brown et al. [12, another way to understand technical debt is to characterize the difference between the current state of a software system and some "ideal" state, in which the system is perfectly optimized in a particular environment. This gap includes items that are typically tracked in a software project, such as known defects and features not implemented. But, it also includes less obvious and less visible aspects, such as architecture, code decay, and outdated documentation [12].

Theodoropoulos et al. [100] propose another definition of technical debt: "Technical debt is any gap within the technology infrastructure or its implementation which has a material impact on the required level of quality." [100]. He explained that "required level", will vary from one environment to another, depending on the situation and timing of each context.

Allman [1] highlights that technical debt is the gap between factors such as lack of time, cost of tools and good development practices. Every time you implement a quick fix to solve a problem but you do not adhere to good practices, you are probably contracting a debt.

McConnel created other definition of technical debt: "A design or construction approach that's expedient in the short term but that creates a technical context in which the same work will cost more later than it would cost to do now (including increased cost over time)." [61].

In the Third Workshop on Managing Technical Debt in 2012 a map of the "technical debt landscape" was created, Figure 2.2 [51]. According to Kruchten et al. [51], this approach helped to identify those kinds of potential technical debt which could not possibly be detected by tools, such as architectural debt or technological gaps. 
Figure 2.2: Technical Debt Landscape

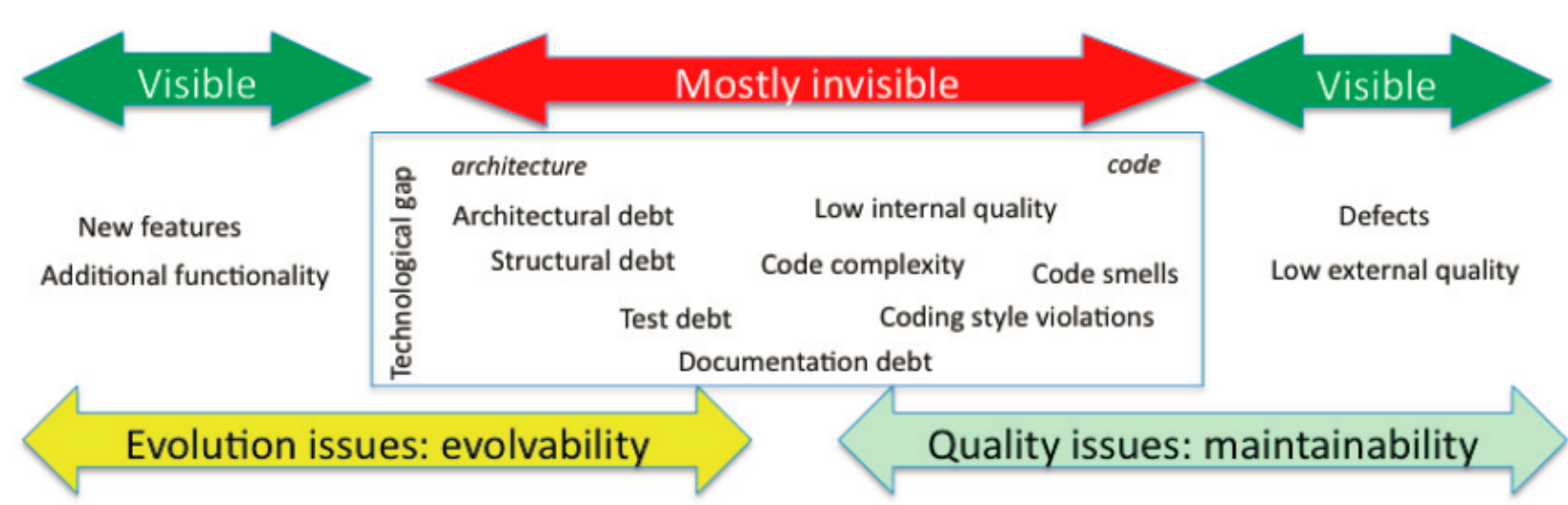

Source: 52

In this Figure 2.2 we can observe visible elements, such as new functionality to implement and defects to fix, and not so visible elements, such as code smells. On the left side, we are dealing with the challenges of evolution and on the right side, we are dealing with quality issues. The Figure 2.2 shows on the right side that the technical debt causes can be: negligence, lack of technical skills, inadequate process, non-systematic verification of quality, or incompetence. Kruchten et al highlight that we only deal with code analysis on the right side the box. On the left side, we tackle critical activities that could incur debt, such as gaps in technology and architecture [51]. Gaps in technology can lead to technical debt, even though a correct decision is made, as a result of the evolution of the context. In this case, technical debt is contracted due to technological obsolescence, change of environment, rapid commercial success, or the advent of new and better technologies. According to Kruchten et al. [51], in other words, it happens because of the invisible aspects of natural software aging and evolution. The architecture is part of the landscape because together with other activities such as documentation and test, it plays a relevant role in software development and can increase technical debt considerably. Kruchten et al. [51] proposed to limit the definition of technical debt to the invisible elements in Figure 2.2 (items in the rectangular box) including the invisible aspects of the software evolution and quality [51].

Brown et al. [11] created a way to improve people's understanding of what technical debt is. For this, a board game called "Hard Choice" has been developed, where players have to make choices that simulate design decisions. The objective of the game is to discuss technical debt and investments in a project in the business context. The game seeks to provide an understanding of how to assess software changes with respect to technical debt costs, both short and long-term, and how individual and collective strategies change throughout project evolution. This game is useful to help teams have a better understanding of what technical debt is in software development projects and to clarify to those involved in the project, what is and what is not technical debt.

In 2016, a group of technical debt researchers [4 met in Dagstuhl, Germany, to discuss technical debt in software engineering, in particular, to standardize the metaphor definition, set a research roadmap, etc. In this Workshop a definition of the technical debt metaphor was proposed:

"In software-intensive systems, technical debt is a collection of design or implementation constructs that are expedient in the short term, but set up a technical context that can make future changes more costly or impossible. Technical debt presents an actual or contingent liability whose impact is limited to internal system qualities, primarily maintainability and evolvability." [4]

In all definitions of technical debt the authors consider as a technical debt those immature items of software that are directly linked to the code. And the others items that may be a problem but 
are not directly connected with the code should not be considered a technical debt item. They also emphasizes that a debt can be used in a short-term as a strategy. And the problem is when the debt incurs in interest, more time the debt is not paid off, project interest rates can rise.

\subsection{Technical Debt Characteristics}

McConnell 60] characterizes technical debt into two types: unintentional and intentional. Unintentional, or involuntarily debt, which is a non-strategic debt, such as low quality work. Sometimes this debt can be created when the developers do not satisfactorily perform a rewrite of a platform that was already indebted and thus only introduced more debt in the code. Intentional debt, on the other hand, commonly occurs when an organization makes a conscious decision, for example, to deliver fast. A typical and convincing phrase is: "If we cannot get this release out on time, there will not be the next release." This leads to decisions like: "We do not have time to update these two databases, so let's code them now and synchronize them later" [60]. McConnel [60] divides intentional debt into two types:

- Short-Term Debt: usually incurred tactically or reactively, to solve an immediate problem. It is expected that this kind of debt will be paid off quickly.

- Long-Term Debt: is debt that is incurred strategically and proactively, for instance, investing in new capital equipment. This kind of debt can be carried for a few years longer.

Fowler [35] used a slightly different set of concepts to describe different types of technical debt. He defined two dimensions, thus creating four quadrants to classify technical debt [35], as can be seen in Figure 2.3 .

Figure 2.3: Technical Debt Quadrant

\begin{tabular}{l|c}
\multicolumn{1}{c|}{$\begin{array}{c}\text { Peckless } \\
\text { "We don't have time } \\
\text { for design" }\end{array}$} & $\begin{array}{c}\text { "We must ship now } \\
\text { and deal with } \\
\text { consequences" }\end{array}$ \\
Deliberate & \\
\hline Inadvertent & \\
"What's Layering?" & "Now we know how we \\
should have done it"
\end{tabular}

Source: [35]

Fowler explains that technical debt is Prudent and Deliberate when the team knows that they are incurring a debt, for instance, as a strategic decision like time to market. Reckless Deliberate technical debt is a mess, and happens when the team makes a conscious decision among poor choices that can result in high interest and/or a long-term to be paid [35]. Technical debt that is both Reckless and Inadvertent is that debt that is incurred negligently. It is considered high risk because developers assume this kind of debt without realizing it. It happens when, for example, debt 
is incurred due to some technical deficiency of the team 35. Prudent and Inadvertent technical debt is common for teams that developed the software in the best way they could at the time. But since they are continuously learning, they conclude that they could have made it better [35].

Finally, the technical debt conceptual model, Figure 2.4 creates a map to understand the relationships between technical debt and other software development concepts [4].

Figure 2.4: Technical Conceptual Model

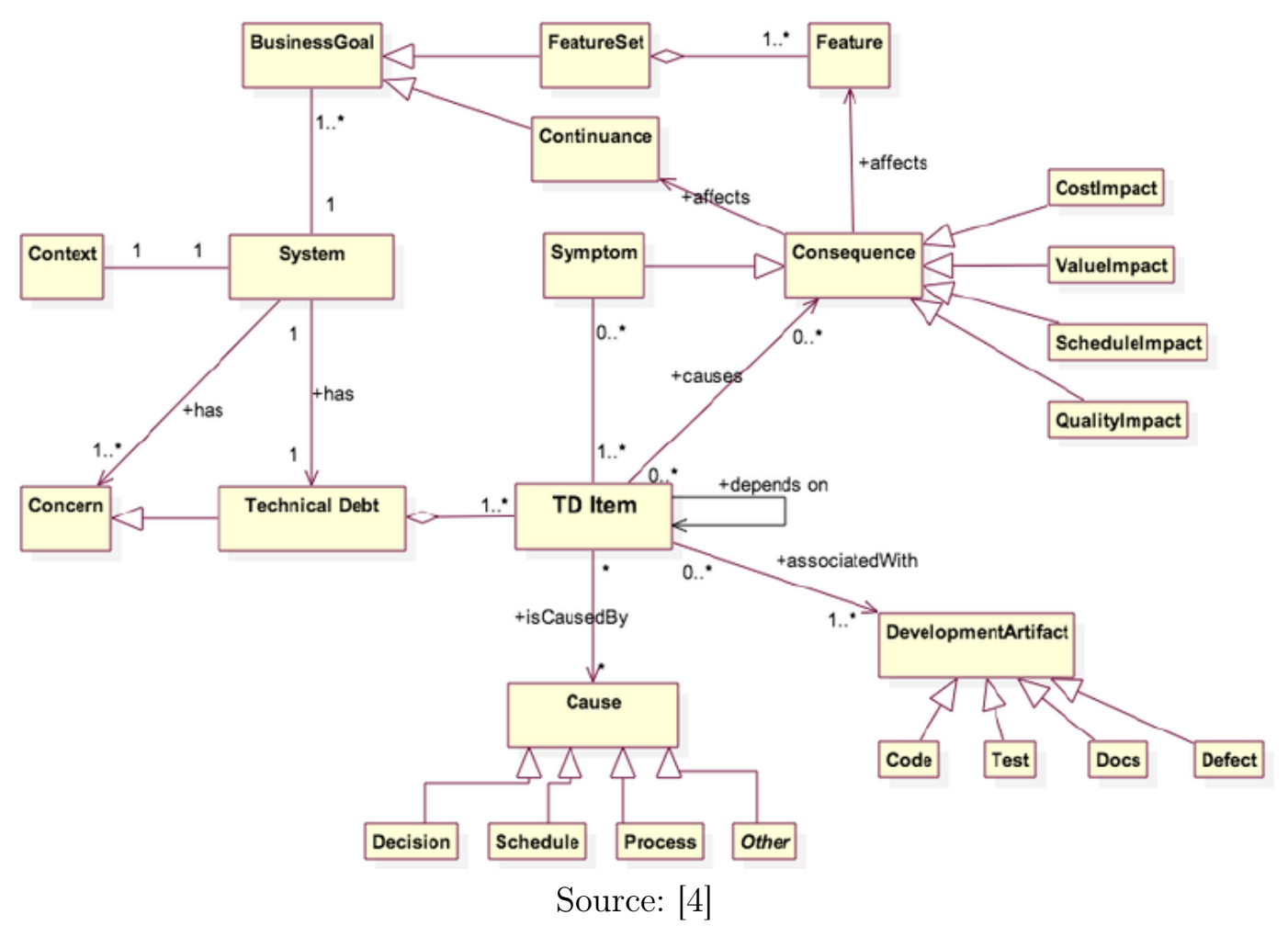

In this model, it is possible to see that technical debt is one of the many concerns related to a software-intensive system [4]. Technical debt items are directly connected to the development artifacts, such as code, test, documents, and defects. Contracting these technical debt items results in some consequences such as lower system quality, difficulties with keeping the schedule, increasing the cost of future changes. These consequences directly affect any software feature and also affect the business goal. These technical debt items generate symptoms that could help the team to identify them. The technical debt is contracted due to reasons such as a process, a decision, an action (or lack thereof), or an event. The causes that motivate the existence of technical debt existence can be: schedule pressure, unavailability of a key person, lack of information or other [4]. Avgeriou et al. 4] emphasize that this model does not include the activities to manage technical debt and the causes that may or may not be input to measurement and analysis.

The study of Izurieta et al. [49] discuss this technical debt conceptual model, Figure 2.4, and provide a brief history, summarizing current research, and showing the research subject challenges and gaps.

The technical debt characteristics helped the teams to understand the boundaries of the technical debt metaphor. It helps them to identify whether or not the problem they are facing is a technical debt. In addition, the analysis of the technical debt characteristics can show for the teams that technical debt items are not always intentional.

\subsection{Technical Debt Properties}

In the literature, there are a number of lists of properties that should be analyzed and used when one decides to consider technical debt. The technical debt properties were considered in our 
study especially when we created the template defining which technical debt information the teams would be collected.

Brown et al. 12 presented some of these properties:

- Visibility: significant problems arise when technical debt items are not visible. In many cases, the technical debt items are known by someone, for instance: "... I know that I broke the encapsulation to implement a feature before the deadline..." [12], but this information was not visible to the other team members that eventually will have to pay for it.

- Value: in the financial field, when technical debt is correctly managed could be used to aggregate and generate value, as a strategy. The value is the gap between the actual version of the system and the ideal delivery state to the customers [12].

- Present Value: the present value of the cost incurred as a result of the debt, including the time to impact and the uncertainty of impact, must be mapped to the overall cost-benefit analysis [12].

- Debt accretion: accumulating some technical debt could result in system depreciation, consequently impacting the code quality and can lead to an irreparable state [12].

- Environment: in software engineering projects, the debt is related to a given or assumed environment [12].

- Origin of debt: it is important to keep a distinction between the strategic debt from those contracted unconsciously, that occurs sometimes as a result of poor practices. It can also be caused by environmental changes in a way that creates an incompatibility that reduces the system value [12].

- Impact of debt: it is important to know the elements that need to be change to repay the debt [12.

Brown et al. 12 also defined some properties as technical debt measures:

- Principal: the estimated cost of eliminating debt [1].

- Interest probability: the probability there will be noticeable consequences of the debt.

- Interest amount: the cost that was added in the software project because the debt was contracted [12].

Falessi et al. [30] discussed the interest probability and emphasized that it is difficult to define because it depends on knowledge of the future events.

In another paper, Guo and Seaman 42 described a list of technical debt items, each of which is described by the properties in Table 2.1:

First, we have to attribute to all technical debt items an ID. After it is important to put the Date that the technical debt was identified or contracted and the Responsible person for it. The technical debt Type categorizes if it is a documentation debt, or design debt, or one of several other types. The Location of the debt points to its location in the code or another document. Also, it is important to add a brief Description of the technical debt, the estimated Principal, that is the value of the cost to fix it now. The Expected Interest Amount and Interest Standard Deviation are used to define the extra work the team will have until the item is paid. Finally, the team could fill in the Correlation with other items, if that item it is connected with other debt [42].

It is important to highlight that when the software development team starts to consider technical debt they can define measures for Principal, Expected Interest Amount and Interest Standard Deviation, based on their experience. When we started to identify the debts we followed these items suggested by Guo and Seaman [42]. 
Table 2.1: Technical Debt Identification Items [42].

\begin{tabular}{|l|l|}
\hline ID & 37 \\
\hline Date & $31 / 03 / 2007$ \\
\hline Responsible & João Developer \\
\hline Type & Design \\
\hline Location & X Component, Functionality Y \\
\hline Description & $\begin{array}{l}\text { The implementation of the Y function violates some de- } \\
\text { sign elements. }\end{array}$ \\
\hline Principal & 1.5 person-day \\
\hline Expected Interest Amount & 0.5 person-day. \\
\hline $\begin{array}{l}\text { Interest Standard Devia- } \\
\text { tion }\end{array}$ & 0.3 person-day. \\
\hline $\begin{array}{l}\text { Correlation with other } \\
\text { items }\end{array}$ & $\begin{array}{l}\text { Item 12: 0.9 (it is very likely that this item will incur } \\
\text { interest if debt item 12 needs extra work to complete, } \\
\text { and vice versa.). }\end{array}$ \\
\hline
\end{tabular}

Besides the properties related to the two studies above, Li et al. [55] added four new additional properties that are: name, context and propagation rules, and intentionality.

$\mathrm{Li}$ et al. [55] analyzes many studies (included the studies related above) and found the list of technical debt properties in Figure 2.5 [55]:

Figure 2.5: Technical Debt Properties

\begin{tabular}{ll}
\hline Field & Description \\
\hline ID & A unique identifier for a TD item \\
Location & The location of the identified TD item \\
Responsiblefauthor & The person who is responsible for repaying the TD item \\
Type & The TD type that this TD item is classified into, e.g., architectural TD \\
Description & General information on the TD item \\
Date/time & The date or time when the TD item is identified \\
Principal & The estimated cost of repaying the TD item \\
Interest amount & The estimated extra cost of tolerating the TD item \\
Interest probability & The probability that the interest of this TD item needs to be repaid \\
Interest standard deviation & The estimated difference between the estimated interest amount and the actual \\
& (future) interest amount \\
Correlations with other debt items & Relationships between this TD item and other TD items \\
Name & The name of a specific type of TD in a TD item (in S39, a TD item may include \\
& multiple TD types) \\
Context & A certain implementation context (e.g., programming language used) of a specific \\
Propagation rules & TD type in a TD item. \\
Intentionality & How this TD item impacts the related parts of the software system \\
& Intentionally or unintentionally incurred \\
\hline
\end{tabular}

\section{Source: [55]}

These properties may help software development teams to start considering technical debt and make it visible. Also, the understanding of the technical debt meaning and relevance is well established and categorized, as we can see in the Li et al. paper [55].

\subsection{Incurring Technical Debt}

Technical debt is incurred due to some reasons. The studies below gave some examples of these reasons. They are crucial in the process of identifying why some technical debt items have been incurred. In the cases we analyzed the historical data, the insights from this study help the team members to define each project and each moment of the project the teams could have been incurred 
in technical debt.

Technical debt can be incurred as an investment 11 such as, for strategic reasons 60. The primary motivation might be due to a belief that the cost of doing it now is less than the cost to do it in the future. McConnell gives several other reasons [60]:

- Time to Market: when the time to the market release is fundamental[60].

- Preservation of Startup Capital: when the company chose to delay the payment of the technical debt items for raising more money [60].

- Delay Development Expense: once a system is retired all the technical debt items of the system are retired with it. Thus at the end of lifecycle of the system, it becomes more and more difficult to justify the investments for paying technical debt [60].

In the Managing Software Debt book, Sterling [95] relates some well-known patterns that directly lead to in technical debt. He describes the following patterns:

- Schedule pressure: often times the team has to meet the expectations of management, due to many reasons, such as: scope creep, new tasks are added to the release without removing scope; third-party estimates, a technical leader or architect defines the estimates and the actual team has to comply with these estimates; change in team makeup, after the release started team members are changed, removed, included; artifact of the estimation process, estimation is a guess exact commitments are made based on it and late integration, when in the release cycle the integration happens late [95].

- Duplication: lists many reasons that causes duplication; lack of experience of part of team members, copy-and-paste programming, pressure to deliver and conformity to existing software's poor design [95].

- Get it "Right" the First Time: it can create a complex solutions with unused functionality that must also be maintained into the future [95].

Technical debt also can be incurred to accelerate development. Buschmann [13] described an example, "...the architects knew about the potential performance threat and intentionally incurred the debt to accelerate development..." [13. Codabux and Williams [15] emphasizes that technical debt can be incurred due to a technical decision, such as architecture decisions.

Lim et al. [57] also described some examples of the motivation to incur technical debt, such developing a working prototype to secure funding from investors and Meeting timeline to integrate with a partner product before release. Furthermore, in that study, they found that incurring technical debt is indirectly or directly related to the customer. One of the reasons is because when the customers do not know exactly what they want, the requirement implemented could not reflect what they needs. Another reason is uncertainty over the future as the coming directions of the market, it is too hard to know how the market will be after many years [57]. Besides that, the customer is not usually willing to wait days for a 'simple' planning.

However, the study of Spínola et al. [93] showed that many practitioners found many technical debt items unintentional [60], and can be contracted due to several reasons such as:

- Lack of Knowledge: when a junior programmer developed something not good enough [60].

- Tradition: when the team never changes the way they do something because it has always been done in a fixed way [30].

- Organizational constraints: the company has some rules that no one can change [30].

- Culture or personal feelings: when a developer never comments the code [30].

- Political issues: whether the team decides to use another tool, it will impact those members that use the current tool and they would not want to change [30. 
- Conflicting goals: when one department prefers one kind of technology, and another department prefers another, the department that has more power will decide, the decision may not be based in a technical analysis [30.

- Technological Gaps: it occurs when the team decides to use specific technologies and due to changes in the business, the technology becomes obsolete [51].

- Strong arguments of an Expert: in the Siebra et al. [89] study, they identify that in a particular case, a technical debt was contracted, because a team member that was expert in a specific technology had strong arguments to use a tool and no one argued against it, the choice resulted in an important debt [89].

In addition, in the Lim et al. [57] research, they identified (in that case) that technical debt was often inserted because of decisions made by high-level technical/non-technical managers. Furthermore, Martini et al. [59] did a study about understanding the causes of architectural technical debt. As a result he identified that technical debt was contracted due to the following reasons:

- Business factors: such as time pressure, market evolution and the uncertainty of use cases in the beginning. Also could be the features priority over product, split of the project budget and maintenance budget boosts the accumulation of debt. To summarize, decisions made to prioritize others business tasks.

- Design and architecture documentation: lack of specification results in misinterpretation by the developer's code.

- Reuse of Legacy/third party/open source: part of the other software that is included after in the definition of the architecture.

- Parallel development: when different teams work on the same project, or when a team works at the same time in distinct projects, it may imply in different patterns.

- Effects of Uncertainty: for instance, lack of awareness about the dependencies of the specific architectural technical debt with the others parts of the software.

- Non-completed Refactoring: if the team decides to do a refactoring to pay a debt and does not finish it, it may result in contracting another debt.

- Technology evolution: when technology becomes obsolete.

- Human factor: as reported in Siebra et al. [89], McConnell [60] and Falessi et al. [30, develop software is an individual task and it is directly related to factors such as decision due to inexperience, careless, ignorance, error-prone situations and so on [59].

To avoid incurring in some technical debt Rubin [79] suggests practices as: continuous integration, test driven development, automated testing, and refactoring.

These techniques can reduce the number of technical debt incurred, but will not solve and/or mitigate all the technical debt problems. So, it is important that teams analyze and consider the historical data of the project to identify the recurrent causes that lead to debt. Thus, it is possible to define techniques and metrics to mitigate a certain type of debt.

Therefore, most authors suggest that time (e.g., time to market, schedule pressure), human factors (e.g., culture or personal feelings) and business factors (e.g., market evolution) are one of the most prevalent factor for incurring technical debt. In the studies we conducted, the main reasons of the technical debt incurrence were business decisions and human factors as time, lack of knowledge and a mandatory decision of the business. 


\subsection{Impact of Technical Debt}

Sometimes technical debt can be necessary, but it is important to know that it may compromises and impact some parts of the project. Sterling [95] emphasizes that there is a cost of not addressing technical debt, the more time the developers ignore the technical debt, the cost of software maintenance increases [95]. According to Brown [11], technical debt creates important long-term problems, such as increased maintenance cost. Buschmann [13] gives an example of a debt contracted by the architect team to accelerate the development. In this case, the effects of the interest surprised them, resulting in business decrease and higher performance tuning costs [13].

According to Highsmith [44, technical debt can severely impact the development speed. In Figure 2.6, he explains that technical debt increases the cost of change over time, leading to software that becomes almost unmaintainable.

Figure 2.6: Technical Debt Cost

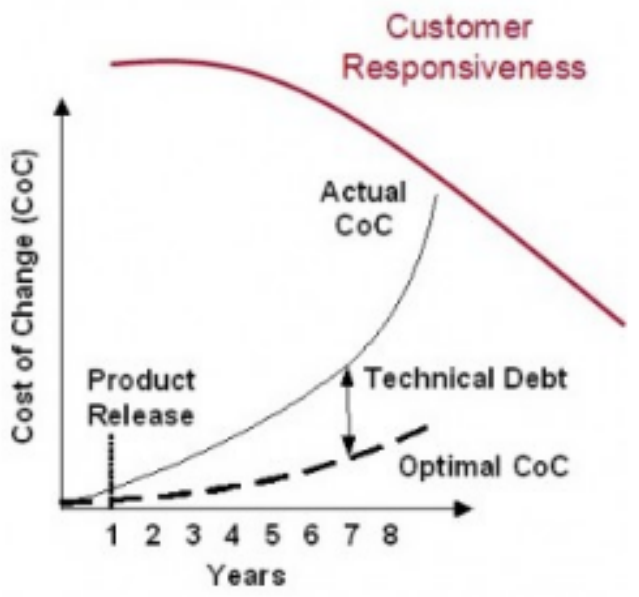

Source: 44]

Furthermore, it impacts the current value and the future value of the software by slowing down the delivery process. The first continuous line shows how software degrades over time and severely impacts customer responsiveness. The second dotted line, shorter-term (by iteration) scale, shows how technical debt can seriously affect development speed very quickly [44]. Then, it could affect the ROI of the project because it delays benefits and costs more to implement 44. If the technical debt increases, development speed decreases, customer satisfaction decreases, and predictability of the results decrease [44].

Other results of contracting a debt were presented in Siebra [89] study. They analyzed three scenarios where the technical debt items were contracted and related the impact on their projects. In this case, it was clear that ignoring technical debt can result in a high-cost [89]. According to Allman [1, in some areas, such as security, the impact of technical debt can be a disaster. Also, he listed the impact of technical debt on different areas:

- Customer: is one of the most badly affected by the technical debt. The company can lose business and customers when the software development teams cannot submit their work on time.

- Help Desk: they are affected because of the poor quality of the software, such as design interface, bad or no documentation, slow programs, etc. The fact is whether the customer has a problem he calls them to complain. And the help desk often has no contact with who developed the software, so they hear the complaint but often can not solve the problem.

- Operations: who have to maintain everything working, they have to correct bugs resulting from the technical debt. 
- Engineers: they are the people who write the code and who inserted the debt. Also, they have to maintain the code most of the time. The technical debt affects their lives directly because in a long-term will be difficult for them to produce a code with high level of quality.

- Marketing: "...people often have to take the brunt of customer displeasure..." [1, when the functionality of the software contains errors, they are the ones on the wrong side. Because they were the ones who sold the software commonly promising that it would work without errors. Moreover, when a customer requests a new feature the marketing staff are pressured to have it delivered soon.

- Management: if the management chooses to favor another sector, for example, marketing. To deliver the software quickly, it could motivate the team to incur a debt[1]. So, the management assumes the technical debt without understanding its cost. And the technical debt often results in some cost.

In most cases, debts have directly affected the code quality and it resulted in different impacts in the product and on business - such as customer dissatisfaction - and on the team itself that have to deal of high level of pressure. The debts identified in our study also impacted primarily on code quality, on business, the stress level of the team and the customer.

To Curtis et al. 22] technical debt can make software less efficient, less scalable, harder to improve or more penetrable by hackers. According to them, technical debt is the result of the poor structural quality and affects business as IT cost and business risk [22].

Lim et al. [57] said that incur technical debt result in increased cost of maintenance. If the system is unpredictable, it is hard to estimate the amount of time and effort required to make changes to the software [57]. Also, in this study, Lim related other examples of technical debt impact, such as; system instability, unexpected behavior, or what might be described as "fragility", bad product's performance and low reliability. Therefore it can create a poor customer perception and can make their customers angry or unhappy [57].

Furthermore, Crispin shows that in agile teams, if the teams do not manage technical debt, the code probably will not be well designed, well written, easy to maintain, or supported by automated tests [6]. Thus, when the team will maintain that code, they will spend much more time to understand what happened before. Also, if the code does not have enough test coverage, the team may spend a large amount of time doing the manual test. So, it leads to assume a high risk because it results in so many unknowns [6].

Hummo et al.[108] did a study at Finnish software company to understand the causes and effects of technical debt. So, they discovered that most of the technical debt items were a result of intentional decisions to reach deadlines. Also, they summarize some causes and effects of technical debt: intentional causes of technical debt -pressure to the development team, lack of time given for development, complexity of the source code, business decisions (lack of technical knowledge and communication challenges), unintentional causes - lack of coding standards and guides, junior coders, lack of knowledge about future changes, lack of documentation, short-term effects of technical debt - time-to-market benefit and increased customer satisfaction, long-term effects of technical debt - extra working hours, errors and bugs, customer dissatisfaction, the complexity of the source code.

\subsection{Technical Debt Management}

Since some technical debt is inevitable, projects have to deal with it and manage it. Each company has a different understanding of the usefulness of the technical debt items, and they can use several different strategies to work with technical debt. Some want to avoid taking any type of debt, others see debt as a useful tool. However, according to studies of McConnell [60], unlike the financial debt, technical debt is much less visible, and so people tend to ignore it more easily. The most frequent reason for developers avoiding the debt is the challenge of communicating the 
existence of the technical debt to business people and explaining what it entails and why it needs to be repaid in the future. McConnell [60] related that it is hard to convince business people to use investments to pay the technical debt items.

According to Brown et al. [12, there are several uncertainties about the management of technical debt. Neil et al. [27] emphasize that there is no explicit standard approach for managing technical debt, but, in many cases, there is some management of technical debt within existing processes [27. Most studies are focused on a technique and/or specific way or property for technical debt management. Other studies are focused on managing some types of technical debt. It is important to consider these studies when the teams will plan how they will deal with technical debt identification, measurement, monitoring and management seeking to use those that best fit the reality of each team and each company. Six studies have a model, or framework for technical debt management which consider all technical debt management activities. Because of that, we compared them to our model in Chapter 7.

According to Allman [1], managing, understanding and communicating technical debt can make a difference in the success of the project in the short and long-term.

Technical debt management involves deciding between a focus on software value versus on cost throughout the decisions made in the development cycle [67]. Technical debt is a balance between value and cost with the main objective of having a return on the investment. So it is important to consider that technical debt has a lifecycle. This lifecycle starts at the time the debt is incurred until the time a strategy is defined and implemented to pay or mitigate it and the time spent during this process. Nord [67] concluded that managing technical debt depends on knowing the impact on key requirements and architecture steps [67]. Li et al. [55] believe that it is necessary to manage the cost of technical debt versus the software value [55].

Schimd [84] emphasizes that we have to accept that technical debt free does not exist, then we have to consider and to manage it. He also suggests differentiating potential technical debt from the effective technical debt, and managing it [84].

According to Sterling [95], the first step to manage technical debt is to understand that technical debt exists and is detrimental to the software quality maintenance.

Dos Santos et al. 24] did a case study in an architecture team. They created a board to make the technical debt items visible in a high-level, in this board they used colors (white $=$ green, yellow $=$ light gray and red $=$ dark gray) to define the compliance level of each criterion of the technical debt items and defined some types of technical debt. Furthermore, they used graphics and tools to measure the technical debt level. They concluded that make technical debt visible improved the team's awareness about the technical debt items and it encouraged the teams to pay the debts. They used the Sonar tool to measure the technical debt items. They had a virtual board that they put on a big screen to show how many technical debt items each team incurred, also in this board they showed some quality metrics, for instance, test coverage. Additionally, they used the gamification technique to motivate teams to pay the debts continually. In our studies, we also used boards, TV screen and graphics to make the technical debt visible but we do not use colors to differentiate them. We also identify the impact on teams once they were aware of the technical debt, in Chapter 5 we described it in details.

Bill [21] suggests seven steps to manage technical debt in the Figure 7.12 .

The flow of technical debt management cycle starts with the IT business priorities to quality assurance [21]:

- Set strategic quality priorities, the IT Executives have to determine the quality priorities for each of the application services it offers for the business [21].

- Establish reduction targets and plans, application managers will need to translate the structural quality priorities into specific targets for their application [21].

- Measure Technical Debt, the structural quality flaws, can be managed in many moments of the software lifecycle, for instance, with static analysis tools [21]. 
Figure 2.7: Technical Debt Management Cycle

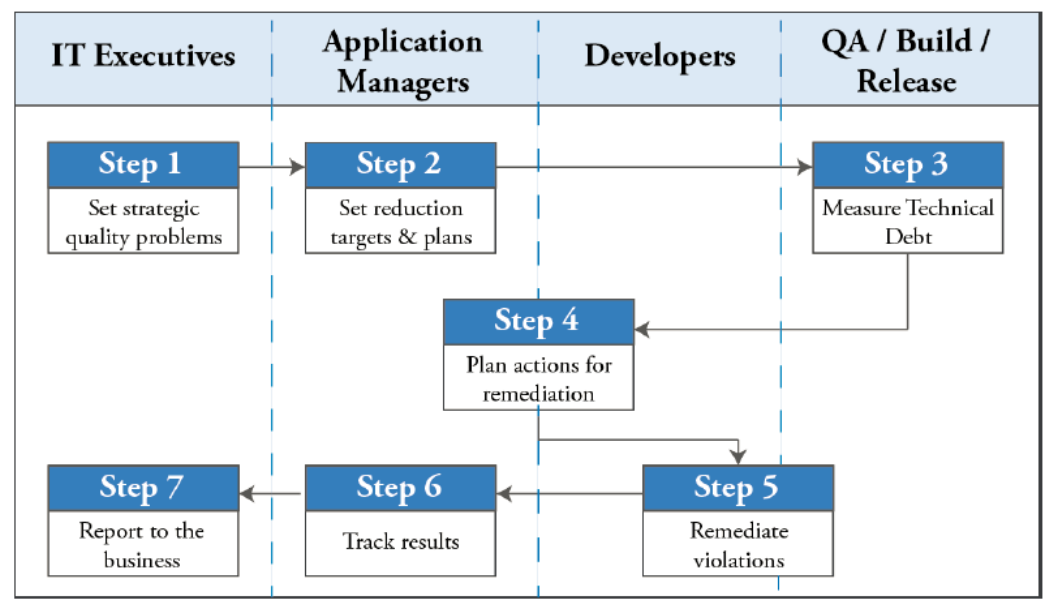

Source: [21]

- Plan remediation actions, in each release planning cycle or sprint, the application manager and development team can use the structural quality analysis from the previous release to prioritize a list of violations for paying some technical debt and achieving the main objectives of the quality [21].

- Remediate violations, the development team pays some debt as a part of their 'normal' development or maintenance activities, these remediation activities can be things such as refactoring [21].

- Track results, the application manager should compare the results of technical debt remediation effort to established targets and share them with the developers. Also, the application manager and IT executives can review the progress periodically [21].

- Report to the business, IT executives could report the status of technical debt to the business [21].

Bavani [6] talks on technical debt management in distributed agile teams. He emphasizes that the management of technical debt directly affects the economics of the software maintenance. According to him, the key to success in the current global economy is building and maintaining software under optimal costs [6]. To better recognize and understand the concept, and to help teams to identify the current state of technical debt, Bavani [6] creates a four quadrant model Figure 2.8.

The quadrant aware and aligned is where the team can see the differences between trivial code quality issues and technical debt. They could also recognize in retrospectives and reviews the technical debt contracted by accident [6]. The quadrant unaware and aligned shows that if teams are unaware of the context of the meaning of the term technical debt, they can consider trivial issues or technical tasks as technical debt. These teams have to improve the awareness of technical debt [6]. Aware and not aligned quadrant occurs when the teams are aware of technical debt but the teams do not have an organized approach for accumulating and managing technical debt [6]. And the unaware and not aligned quadrant is the highest level of risk because they accumulate debt and they do nothing with it [6]. This quadrant helps teams to identify what is the current state of the team on technical debt.

The study of Letouzey [54] suggests making visible the practices to manage technical debt, put it in tools and processes across the Company to produce benefits.

Seaman and Guo [87] suggest a technical debt management approach as can be seen in Figure 7.7.

According to Seaman and Guo [87], to be able to manage the technical debt items it is necessary to first identify them, make it visible and also to identify their causes. After being able to identify and register the debt, they suggest as a second step, to estimate the effort that would be necessary to pay the identified technical debt [87. 
Figure 2.8: Four Quadrant Model

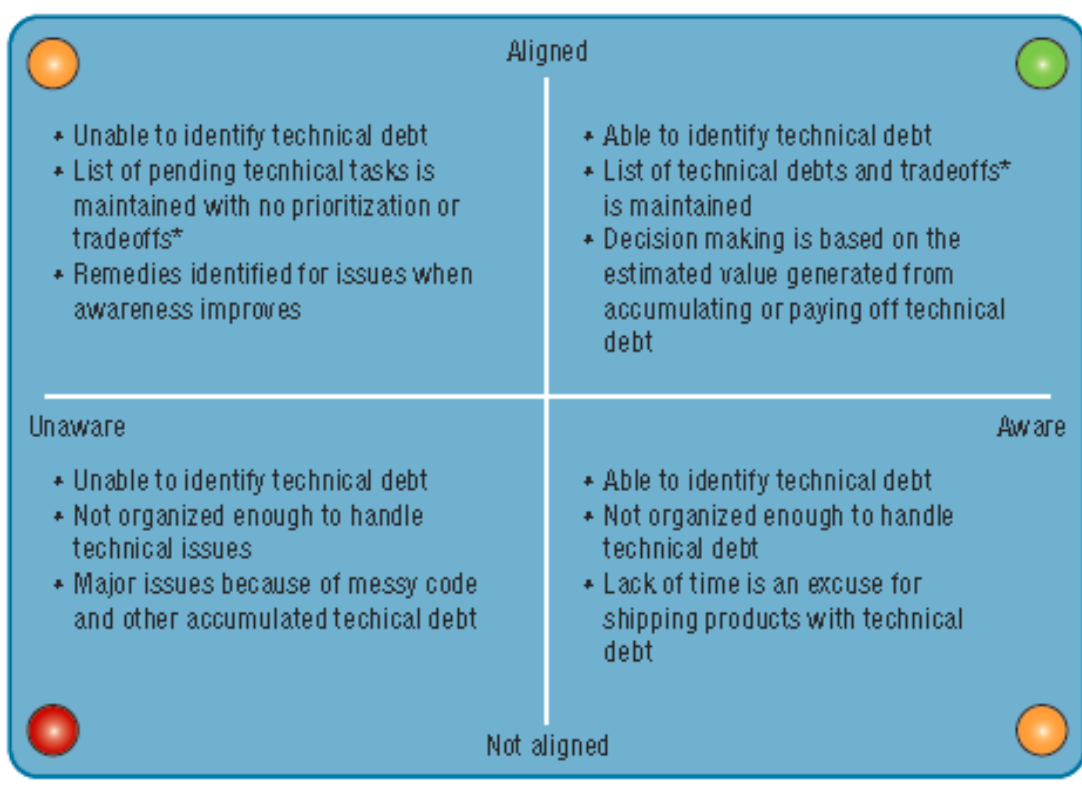

*Tradeoff can be an alternate approach to lessen the impact of technical issues or debt

Source: [6]

Figure 2.9: Framework for Technical Debt Management.

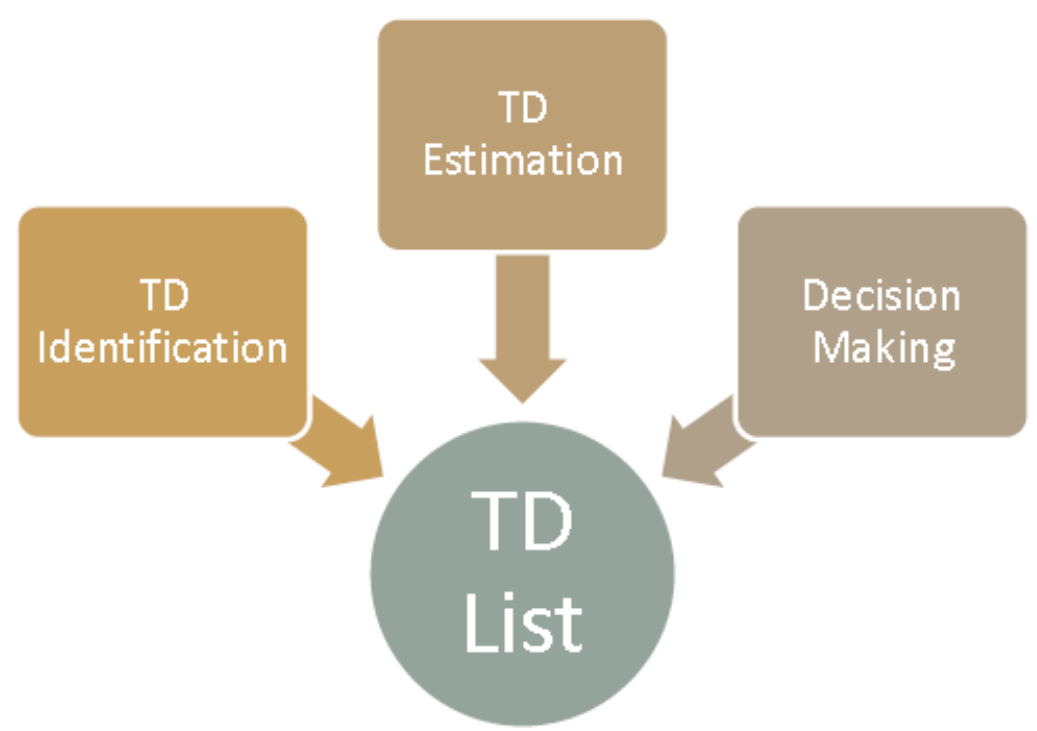

Source: [87]

Once the team has a technical debt list, they should monitor them over the project lifecycle. Thus, there is a constant need to make the decision to pay or keep them under monitoring. This decision is commonly based on the analysis of the technical debt impact on the product versus the cost of paying it in the future [87. This model was the first model created on technical debt management and we followed this model to start and plan our research.

In another study, Rubin [79] suggests a similar approach to technical debt management, as can be seen in Figure 2.10 .

The author points out that to manage technical debt items, it is necessary to manage the accumulated technical debt. The author makes the following analogy: "... if you keep accumulating the technical debt items it is as if you continually borrowed money to maintain your house. One day 
Figure 2.10: Activities for Technical Debt Management. [79].

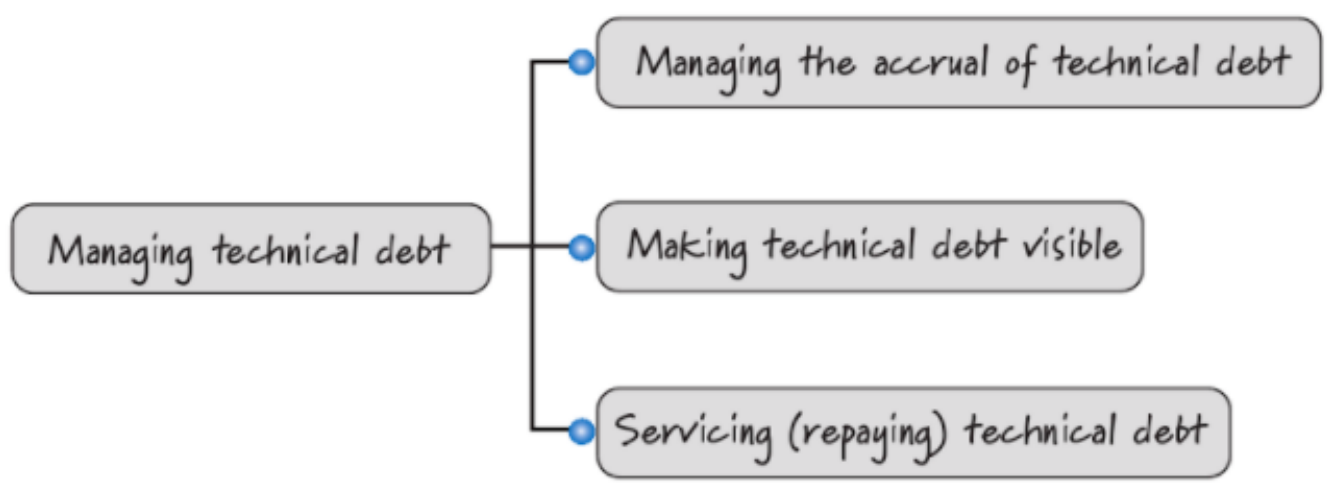

Source: [79]

you will have to stop and say, no more!...", because the consequence will be severe. In addition, to reduce the accumulation of debt over time, the author suggests using good programming practices and having a strong definition of done. Also, it is crucial to understand the economic properties that are involved with technical debt, what is the technical debt cost for the project [79.

The second activity suggested by Rubin [79] is making the technical debt items visible even on the business level. Because according to the author, it is often visible to developers where the technical debt items are in the product, but it is not visible for people at the business level. However, Rubin [79] highlights that technical project members commonly have tacit knowledge of what is the most terrible debt in the product. However, but does not mean that they know the location of the debt and that the debt are visible to the point of being discussed and analyzed.

Finally, Rubin [79] proposes in Figure 2.20 a third activity to perform technical debt management, aimed to help the maintenance or the debt repayment. He highlighted that not all the technical debt items must be paid, but it is necessary to treat technical debt items over time, incrementally [79]. The author [79] mentioned three moments when he recommends not paying off debt: when the product is at the end of this lifecycle, when it is already known that the product will have a short lifecycle and when it is only a prototype that can be discarded [77].

Holvitie [46] discuss the application of technical debt management for software implementations "...including entry points for knowledge discovery, network analysis for overcoming the maintenance challenges as well as the pursued outcomes...". He described the three objectives that were derived to overcome: creating a tool capable of capturing technical debt instances, a static model to automate maintenance for them and integration of the model with the tool to produce a fully functioning management suite. In this study, he used a DebtFlag tool and a statistics model to identify technical debt items and control the propagation of the debts.

Hummo et al.108] suggested some practices to reduce and manage technical debt items such as: refactoring, bug fixing, code reviews, coding standards.

Assunção et al. [23] conducted a study at the Brazilian Federal Administration aimed to manage technical debt. For it, they defined four stage: definition of violations, attribution of values to the pointers and measurement of non-conformance. They used the SQALE model to assess the technical debt items. So, based on the number of violations they calculate the amount of time it was necessary to solve the technical debt items.

Falessi and Voegele [29] emphasizes that without considering (validation and prioritization) the quality rules, it is not possible effectively manage technical debt. Thus, they suggested to have a context-specific validation of the quality rules, once according to them, the tools that provide a static analysis of the code have as a result a huge number of violation and it implies that the effort required for fixing these issues should be higher than the cost of the project.

Sandberg et al. 81] conducted a study at Ericsson Company in two different products, where 
each contained several million lines of code. This teams used many quality metrics to monitor the quality of the code and combine these metrics to create a new metric to manage the quality, for instance, they combine graphics that show how much modules changes and its dependencies. As a conclusion they suggested some principles to proactive manage the software quality, for instance, embrace the technical debt existence, understand the reasons for the technical debt appearance, among others.

Izurieta et al. [48] emphasizes that to manage technical debt it is necessary to create a taxonomy that defines technical debt at the modeling level during software specification. Including defining techniques for its detection, quantification, and removal.

Oliveira et al.[70, managing technical debt in some software projects that using Scrum. They used the Trello tool to create a technical debt backlog and the Vtiger and Jira (each of it in different companies) tools to register the technical debt items. They choose a radon number of technical debt items to estimate, the values were defined by pairs. They also defined a range of value to measure the debts - high, medium and low - for principal, interest amount and interest probability. They also define the equivalence between complexity points to efforts, in hours. The technical debt monitoring was done by the people who estimated the debt. The payment of the technical debt items was defined in the sprint planning meeting, where one team used the five steps of Seaman and Guo's [42] framework and the other team used the five steps suggested by the Lim et al. [57] framework.

In the Ramasubbu and Kemerer [76], they suggested - once the teams had defined the metrics to technical debt management - consider these metrics in three dimensions: customer satisfaction needs, extend of software reliability demanded by business and the probability of technology disruption in a firm's environment. According to the measure of this three dimensions they recommend eight scenarios to technical debt management, as we can see in Figure 2.11.

Figure 2.11: Technical Debt Management Framework

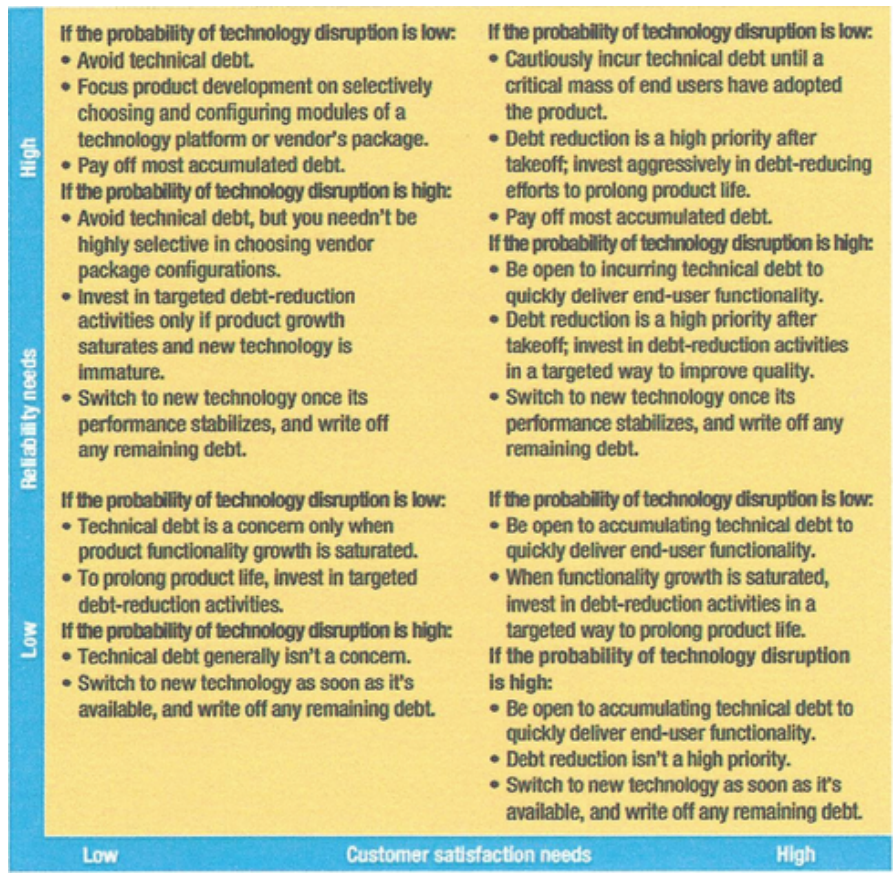

Source: [76]

These scenarios can help teams to identify how and at what level they are dealing with technical debt.

Sanchez et al. [31] conducted a systematic mapping to identify the techniques and methods for technical debt management, focus on architectural debt. They organized a framework listing the elements for technical debt management, as we can see in Figure 7.13 .

They divided the framework in core elements, according to them these elements represent "...con- 
Figure 2.12: Framework for the Elements for Technical Debt Management

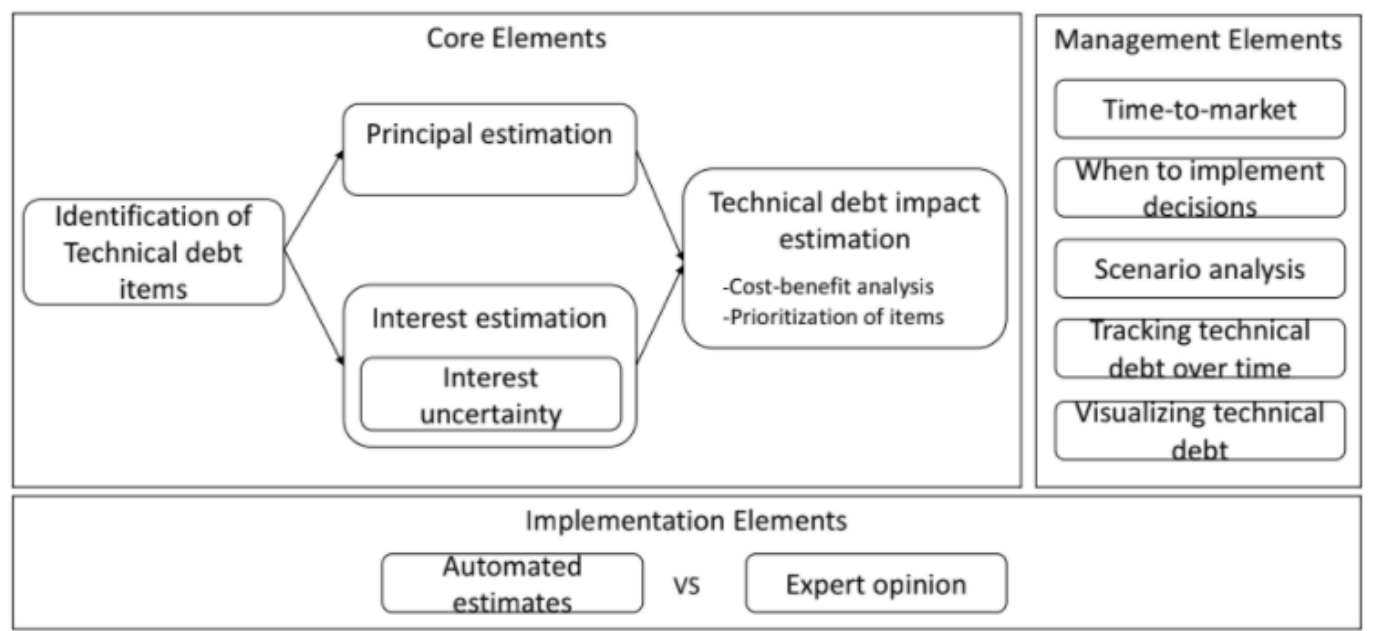

Source: [31]

crete requirements to achieve in order to be able to manage technical debt.". They divided the elements into three groups: implementation elements - focus on estimating the items of technical debt on the system (e.g., principal estimation)-, the management elements - to define what is necessary to identify the debts (e.g., visualizing technical debt) - and the implementation elements (e.g., expert opinion). They also listed the techniques and approaches to support technical debt management, such as tracking technical debt over time.

Harun et al. 43 created a framework to manage and reduce technical debt items based on the cost-benefit analysis (CoBeTDM), focus in the activities: the identification \& assessment, monitoring, quantification \& prioritization, and repayment, as we can see in Figure 7.8 .

Figure 2.13: Framework to Manage and Reduce Technical Debt

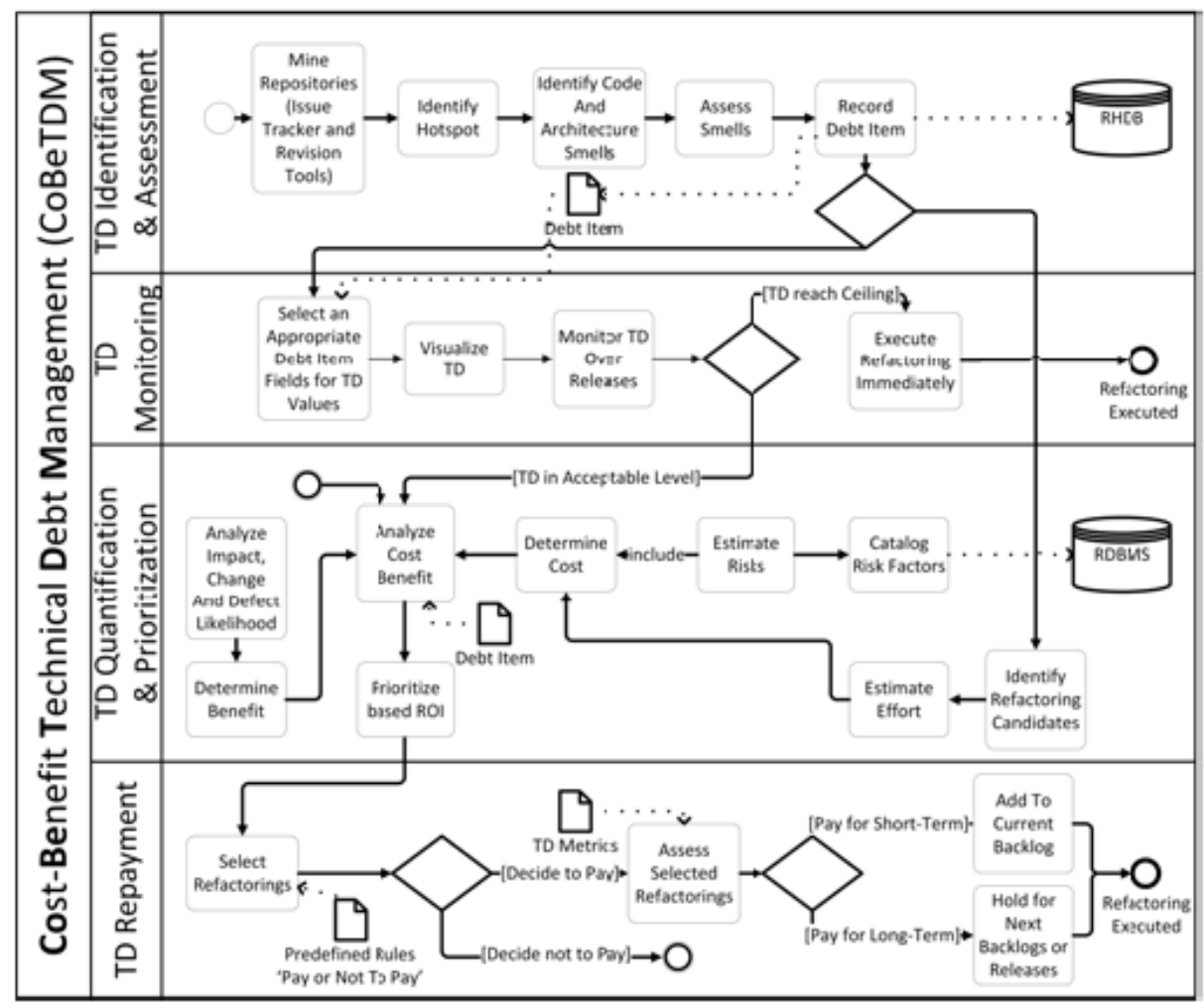

Source: [4] 
In each of theses phases they suggested considering some quality metrics combine with the process decisions. They divided the level into four parts, the first part is technical debt Identification Assessment where they suggested to the first mine the repositories (Issue tracker and revision tool), identifying code and architecture smells and finding frequent changes in the software artifacts, then record and save the data in the database. They used a specific form to collect the information on technical debt item. The second part in their model is Monitoring, where they select an appropriate debt item fields for technical debt values, the visualization of the debts, monitoring over releases and to make the decision whether the debt has to be paid - by refactoring - or the debt has an acceptable level and still be monitored. To visualize the debts they suggested a dedicated dashboard, to conduct the interview with the manager and to develop a risk mitigation strategy to be used if the technical debt reaches the ceiling. In the Quantification \& Prioritization part, they quantified the debt to define a cost-benefit analysis. After the analysis of the technical debt impact, they determine and analyze the benefit-cost (e.g., the less effort of refactoring), this cost is based on determining the cost, risk and estimate effort. As the cost is calculated, the debt is prioritized by the refactoring cost based on the ROI formula - ROI = (Saving Effort and Less Impact of Proposed Refactoring/Effort of Proposed Refactoring). The last part of their model is the repayment, where they have the items selected in the prioritize step, then according to the rules, they decided to pay off or not the debts. Once they choose to pay, the can choose to pay for short-term (Adding the items to current backlog) or pay for long-term (Holding the items for next backlog/releases, to pay off by refactoring.

The study of Huumo et al. [109] investigated eight software development teams to understand how they deal with technical debt. In this study, they discuss each technical debt management activity. They created a framework for technical debt management. In the framework they considered eight activities, for technical debt management: repayment, prevention, representation/documentation, identification, measurement, monitoring, communication, prioritization. For each of these activities they created a level: Practices/tools for activity, responsibility for activity, (Level 1) Unorganized, (Level 2) Received and (Level 3) Organized, as we can observe in Figure 7.10.

In the activity repayment, a team in an organized level make a continuous repayment and achieve the percentage predefined by the team. After, it is important to set as the responsible for these activities the development team and software architect(s), as a practice they recommend refactoring, redesigning and rewrite. In the prevention activity, they suggested that teams in the high level can follow mandatory prevention practices and continuous practices during development. As a practice they suggested coding standards, code reviews, and definition of done. In the representation/documentation activity, they listed that teams in the organized level have it as a mandatory practice. They suggested for it a technical debt backlog list, documentation practice and management tool (Jira, WIKI). In the identification activity, they related that the practitioners with organized level, continuously identify the debts during the development. As a practice, they suggested that the teams save some time for manual code inspection, and using code analysis tools(SonarQube, FindBugs and CheckStyle). To measure and monitor the debts according to them, an organized team continuous measure considering and analyzing data from different metrics such as quality performance including doing it by tools (Jira, Wiki, and SonarQube). Until this activity they recommend as a responsible for the activities the teams of development and architecture. For the activities measuring and monitoring they suggested the software architect(s) and the team manager as a responsible. For the communication activity, they proposed in an organized level, discussions/meetings on technical debt issues with all the stakeholders involved. The responsible for this activity includes development team, software architect(s) and team manager, as a practice they suggest discussion in meetings. Finally, in the prioritization activities, they recommend that a team in a high level, can follow a specific method or model and can use practices such as cost/benefit model, issue rating, to prioritize the debts. As the responsible, they suggested the software architect(s) and the team manager.

In the Vathsavayi and Systã [105] study, they apply a multi-objective genetic algorithm to help developers to define each technical debt it was important to pay, based on the estimation of the 
Figure 2.14: Technical Debt Management Framework

\begin{tabular}{|c|c|c|c|c|c|c|c|c|}
\hline $\begin{array}{l}\text { TDM } \\
\text { activity/TDM } \\
\text { levels }\end{array}$ & TD repayment & TD prevention & $\begin{array}{l}\text { TD } \\
\text { representation/ } \\
\text { documentation }\end{array}$ & TD identification & TD measurement & TD monitoring & $\begin{array}{l}\text { TD } \\
\text { communication }\end{array}$ & TD prioritization \\
\hline $\begin{array}{l}\text { Organized (Level } \\
\text { 3) }\end{array}$ & $\begin{array}{l}\text { Continuous } \\
\text { repayment with } \\
\text { monthly } \\
\text { assigned } \\
\text { percentage of } \\
\text { the development } \\
\text { tasks. }\end{array}$ & $\begin{array}{l}\text { Mandatory } \\
\text { prevention } \\
\text { practices used } \\
\text { by the team. } \\
\text { Continuous } \\
\text { practice during } \\
\text { development. }\end{array}$ & $\begin{array}{l}\text { Documentation } \\
\text { is a mandatory } \\
\text { practice in } \\
\text { development, } \\
\text { Issues are } \\
\text { documented in a } \\
\text { separate TD } \\
\text { backlog. }\end{array}$ & $\begin{array}{l}\text { Continuous } \\
\text { identification } \\
\text { conducted } \\
\text { manually and/or } \\
\text { with tools } \\
\text { during } \\
\text { development. }\end{array}$ & $\begin{array}{l}\text { Continuous } \\
\text { measurement } \\
\text { during } \\
\text { development. } \\
\text { Data analysis } \\
\text { (various data } \\
\text { used (e.g. } \\
\text { quality. } \\
\text { performance)). } \\
\text { Assisted with } \\
\text { tools. }\end{array}$ & $\begin{array}{l}\text { Continuous } \\
\text { monitoring } \\
\text { during } \\
\text { development } \\
\text { with various } \\
\text { data (e.g. quality. } \\
\text { performance). } \\
\text { Tools used to } \\
\text { support. }\end{array}$ & $\begin{array}{l}\text { Continuous } \\
\text { discus- } \\
\text { sions/meetings } \\
\text { about TD issues } \\
\text { with all the } \\
\text { necessary } \\
\text { stakeholders } \\
\text { involved. }\end{array}$ & $\begin{array}{l}\text { Prioritization } \\
\text { conducted } \\
\text { continuously } \\
\text { during } \\
\text { development, } \\
\text { Prioritization } \\
\text { follows a specific } \\
\text { method or } \\
\text { model. }\end{array}$ \\
\hline $\begin{array}{l}\text { Received (Level } \\
\text { 2) }\end{array}$ & $\begin{array}{l}\text { Repayment } \\
\text { during normal } \\
\text { development } \\
\text { tasks and } \\
\text { previously } \\
\text { identified } \\
\text { repayment tasks, } \\
\text { Repayment } \\
\text { conducted based } \\
\text { on current } \\
\text { needs. }\end{array}$ & $\begin{array}{l}\text { Optional } \\
\text { prevention } \\
\text { practices, Not } \\
\text { mandatory to } \\
\text { use, but } \\
\text { recommended, } \\
\text { Conducted based } \\
\text { on current time } \\
\text { constraints. }\end{array}$ & $\begin{array}{l}\text { Documentation } \\
\text { an optional } \\
\text { practice, but } \\
\text { recommended, } \\
\text { Issues } \\
\text { documented in a } \\
\text { general } \\
\text { development } \\
\text { backlog without } \\
\text { TD id. }\end{array}$ & $\begin{array}{l}\text { Identification } \\
\text { optional during } \\
\text { normal } \\
\text { development. } \\
\text { Conducted based } \\
\text { on current time } \\
\text { constraints. }\end{array}$ & $\begin{array}{l}\text { Measurement an } \\
\text { optional } \\
\text { practice, } \\
\text { Measurement } \\
\text { done with } \\
\text { simple data } \\
\text { (number of TD } \\
\text { issues) from } \\
\text { development, } \\
\text { and the data not } \\
\text { necessarily used } \\
\text { for other } \\
\text { activities. }\end{array}$ & $\begin{array}{l}\text { Monitoring } \\
\text { based on simple } \\
\text { data (number of } \\
\text { TD issues). } \\
\text { Conducted } \\
\text { occasionally. }\end{array}$ & $\begin{array}{l}\text { Discussions/ } \\
\text { meetings } \\
\text { organized only } \\
\text { with some } \\
\text { stakeholders, }\end{array}$ & $\begin{array}{l}\text { Prioritization } \\
\text { based on } \\
\text { hunches and } \\
\text { rough } \\
\text { estimations } \\
\text { based on } \\
\text { previous } \\
\text { experiences. } \\
\text { Prioritization } \\
\text { done in a simple } \\
\text { way without any } \\
\text { specific model. }\end{array}$ \\
\hline $\begin{array}{l}\text { Unorganized } \\
\text { (Level 1) }\end{array}$ & $\begin{array}{l}\text { Repayment not } \\
\text { conducted at all } \\
\text { or only when it } \\
\text { is not possible to } \\
\text { avoid the issue } \\
\text { any longer. }\end{array}$ & $\begin{array}{l}\text { Prevention not } \\
\text { assigned as part } \\
\text { of the } \\
\text { development } \\
\text { practices, } \\
\text { Conducted only } \\
\text { occasionally. }\end{array}$ & $\begin{array}{l}\text { Documentation } \\
\text { not part of } \\
\text { development. } \\
\text { Issues are left in } \\
\text { developers' own } \\
\text { minds and notes. }\end{array}$ & $\begin{array}{l}\text { Identification } \\
\text { practices not } \\
\text { assigned as part } \\
\text { of development. } \\
\text { Conducted only } \\
\text { when issues } \\
\text { occur. }\end{array}$ & $\begin{array}{l}\text { Measurement } \\
\text { not part of } \\
\text { development } \\
\text { practices. }\end{array}$ & $\begin{array}{l}\text { Monitoring not } \\
\text { part of } \\
\text { development } \\
\text { practices. }\end{array}$ & $\begin{array}{l}\text { TD not a topic in } \\
\text { discus- } \\
\text { sions/meetings } \\
\text { and of ten } \\
\text { handled only in } \\
\text { coffee table } \\
\text { discussions. }\end{array}$ & $\begin{array}{l}\text { Prioritization not } \\
\text { conducted, and } \\
\text { decisions done } \\
\text { without } \\
\text { reasoning or } \\
\text { discussions. }\end{array}$ \\
\hline $\begin{array}{l}\text { Responsibility } \\
\text { for activity }\end{array}$ & $\begin{array}{l}\text { Development } \\
\text { team, software } \\
\text { archirect(s) }\end{array}$ & $\begin{array}{l}\text { Development } \\
\text { team, software } \\
\text { architect(s) }\end{array}$ & $\begin{array}{l}\text { Development } \\
\text { team, software } \\
\text { architect(s) }\end{array}$ & $\begin{array}{l}\text { Development } \\
\text { team, software } \\
\text { architect(s) }\end{array}$ & $\begin{array}{l}\text { Software } \\
\text { archirect(s). } \\
\text { team manager }\end{array}$ & $\begin{array}{l}\text { Software } \\
\text { architect(s). } \\
\text { team manager }\end{array}$ & $\begin{array}{l}\text { Development } \\
\text { team, software } \\
\text { architect(s). } \\
\text { team manager }\end{array}$ & $\begin{array}{l}\text { Software } \\
\text { architect(s). } \\
\text { team manager }\end{array}$ \\
\hline $\begin{array}{l}\text { Practices / rools } \\
\text { for activity }\end{array}$ & $\begin{array}{l}\text { Refactoring. } \\
\text { redesigning. } \\
\text { rewriting }\end{array}$ & $\begin{array}{l}\text { Coding } \\
\text { standards, code } \\
\text { reviews, } \\
\text { Definition of } \\
\text { Done. }\end{array}$ & $\begin{array}{l}\text { Technical debt } \\
\text { backlog/list, } \\
\text { Documentation } \\
\text { practice, project } \\
\text { management } \\
\text { tool (JIRA, Wiki) }\end{array}$ & $\begin{array}{l}\text { Time reservation } \\
\text { for manual code } \\
\text { inspection, Use } \\
\text { of code analysis } \\
\text { tools } \\
\text { (SonarQube, } \\
\text { CheckStyle, } \\
\text { FindBugs). }\end{array}$ & $\begin{array}{l}\text { Data from } \\
\text { measurement } \\
\text { tools } \\
\text { (SonarQube) and } \\
\text { data from } \\
\text { project } \\
\text { management } \\
\text { tools (JIRA, } \\
\text { Wiki). }\end{array}$ & $\begin{array}{l}\text { Monitoring tools } \\
\text { (SonarQube). } \\
\text { Project } \\
\text { management } \\
\text { tools (JIRA, Wiki) } \\
\end{array}$ & $\begin{array}{l}\text { Specific TD } \\
\text { meetings, TD } \\
\text { included in } \\
\text { discussion } \\
\text { topics. }\end{array}$ & $\begin{array}{l}\text { Cost/Benefit } \\
\text { model, Issue } \\
\text { rating }\end{array}$ \\
\hline
\end{tabular}

Source: [109]

short-term value (i.e., benefit of focusing on the features) and future investment value (i.e., benefit of paying the debt items).

Chicote [14] suggested a visual thinking technique to make visible technical debt, he called the duct taped technical debt. It consists cutting a piece of duct taping every time the technical debt is identified. These pieces represent some part of the code and the size of the piece should correspond to how big is the debt. The idea is helping the startups to identify the number of debts and how the debts affect the development cycle once their early stage condition can result in the incurrence of many technical debt items.

Ramasubbu and Kemerer 75 integrated a model for technical debt management with the management process for software quality suggested in the project management body of knowledge (PMBOK). They divided this model into three steps: make technical debt visible, perform costbenefit analysis and control the debts.

Besker et al. 9] created a descriptive model focus on manage architectural technical debt (ATD), as we can observe in Figure 7.11. This study focuses on managing activities, approaches, and tools on technical debt in general and not especially on the architectural aspects of it.

This model has four main group: Importance of ATD (Architectural technical debt), ATD Checklist, ATD Impediments, ATD Management. In each of these groups, they listed the related 
Figure 2.15: Technical Debt Management Framework

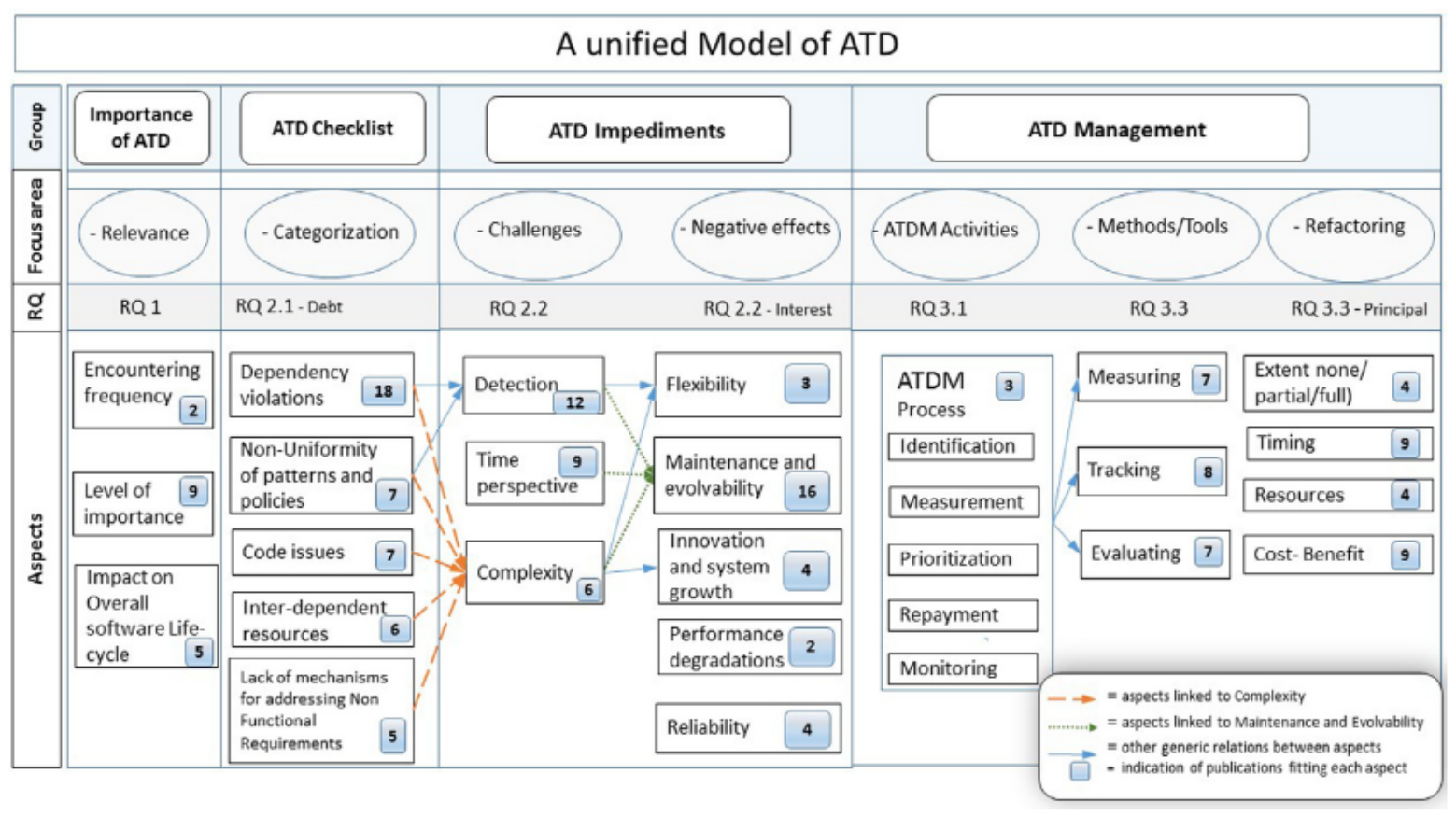

Source: 9

aspects and how they are connected. In ATD importance they listed aspects as an impact on overall software lifecycle. In ATD Checklist they list five aspects of technical debt categorization such as dependency violations. In ATD Impediments they divided into Challenges and Negative effects, for example as a challenge they list detection and as a negative effect of it, maintenance and evolvability. In the ATD Management, they divided into ATDM Activities, methods and refactoring. In this part, they cited Identification, Measurement, Prioritization, Repayment, and Monitoring. For this activities they suggested as a methods/tools, measuring, tracking and evaluating and so to do a refactoring they list four dimensions that have to be considered. In the ATD model, the focus is architectural technical debt and our model the focus is to manage all type of technical debt. They suggest using tools to management activities but also said that tools currently available could not identify all types of architectural technical debt. They emphasize that sometimes is a better option to pay the interest rather than do a refactoring in case of architectural technical debt.

Following the studies of Seaman et al. [88], Rubin [79], Holvitie [47] and Li et al. [55], we organize the next sections in, what is not technical debt, technical debt prevention, technical debt identification, technical debt measurement, technical debt prioritization and decision making, and technical debt monitoring, technical debt communication and tools to support technical debt management.

\subsubsection{What Is Not Technical Debt}

Several times when the teams started to discuss on technical debt, a doubt about what is or not technical debt always comes up. Because of that, it is crucial to consider the studies related below in the moment of the definition of the items that the team will consider as technical debt. These studies provide some suggestions and reflections on the items that may or may not be considered as technical debt.

According to Bavani [6], when teams are unaware of the meaning of the term technical debt, they can consider trivial issues or technical tasks as a part of technical debt item. This occurs because they cannot distinguish between a mess and a design flaw [6]. Furthermore, Curtis [22] relates that the teams have to distinguish technical debt from defects or failures. According to him, failures during test or operation may be symptoms of technical debt. He believes that most of the structural flaws creating technical debt have not caused test or operational failures [22]. 
In the technical debt landscape ( Figure 2.2), Kruchten et al. suggest that not all visible elements are technical debt, such as defects, low external quality, new features and additional functionality [52. In addition, in this study Kruchten et al. [52] emphasize that low external quality that is visible to the user in the form of defects, bugs, and so on, should not be considered as technical debt. Because, for instance, defects have a current impact on the value of the product and technical debt is only felt in the future in the form of additional cost [52]. He also described people debt as a non-technical debt [52. Also, the social debt [98] that occur when the project finished and the people who developed the software and sustaining are lost, affecting how to the new team will deal of the software to maintain and evolve. Although debts such as social debt may result in technical debt, they are not technical debt.

Furthermore, Sterling [95] wrote a book about software debt, and there he describes other kinds of software debt that were not technical debt:

- Quality debt, lack of ability to verify the functional and technical quality of software [95].

- Configuration management debt, when integration and release management become more risky, complex, and error-prone [95].

- Design debt, when the cost of maintain is greater than implementing the code from scratch [95.

- Platform experience debt, occurs when the availability of people to work on changing the software is becoming limited [95].

Li et al. [55] defined as a non-technical debt the items listed in the Figure 2.16. They also highlighted that some studies consider defects as a technical debt [55].

Figure 2.16: Non-technical debt

Non-TD

Defects
Unimplemented features or functionalities
Lack of supporting processes
Unfinished tasks in the development process
Trivial code quality issues
Low extenal quality

Source: 55

Bellomo et al. [8] created a tree to help developers to identify some kinds of technical debt, the purpose is to separate technical debt from other issues, see Figure 2.17.

The decision tree starts with the term, enough info: if the issue does not have enough information they tag it has a non-technical debt, so it has to take the exit D1. If the issue can be classified as a Executable and/or data: then they separate the technical debt items that are directly related to artifacts of the code development, for example, code, test. And they eliminate those kinds of activities related to the project management, for instance, documentation. In this step, they recommend keeping executable artifacts such as code, automated tests, build scripts, poorly structured database record content, duplicate records, etc. They suggest discarding non-executable artifacts such as process issues, policy issues, documentation, and requirements specifications [8]. The next step is classifying according to the Type: if the issue type is a defect, the next step is classifying the defect type, if it is incorrect functionality, for instance, a button does not work in the UI, lack of conformance to specification or an implementation mistake then take the exit D3 because it is not considered a technical debt, these are system errors [8]. The item can be classified with a defect type of design issue, if it limits the correct operation of the system and/or has implications for a quality attribute (e.g., availability, security, performance, modifiability). They suggest 
Figure 2.17: Technical Debt Identification Decision Tree

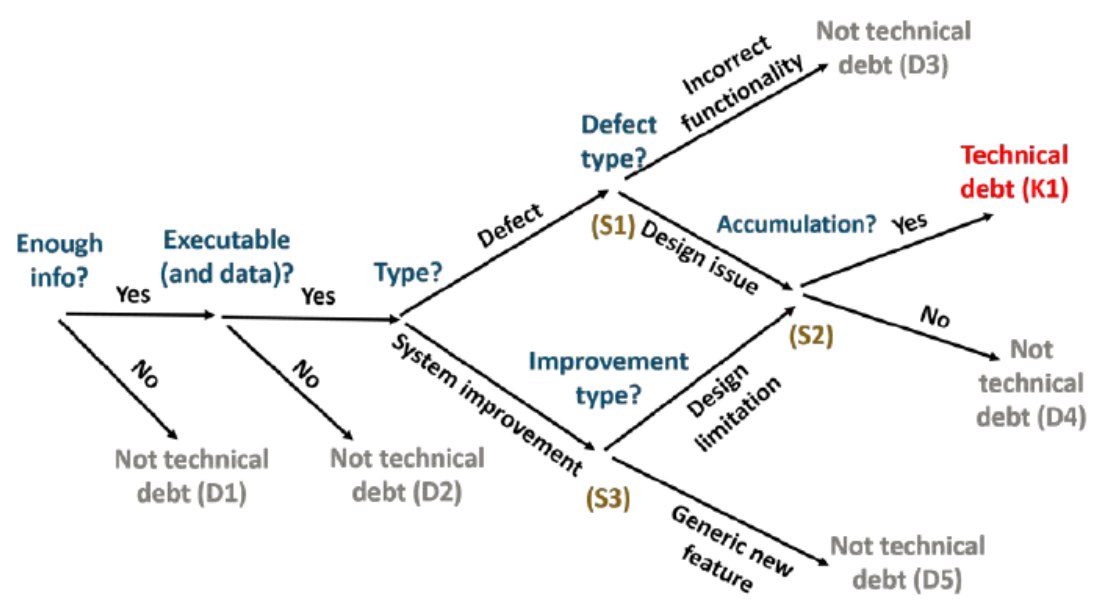

Source: [8]

also including cleanup activities such as removing duplicate code, non-standard binding (assumes duplicating "standard") different implementations, unused classes (dead code), etc. However, if the item type is classified as a System Improvement it is possible to choose between two options; the first is classifying the issue as a new feature and then is not a technical debt item. Or the second option is Design Limitation, to analyze those cases in which the issue is not a mistake or defect but a system improvement to refactor some design limitation, such as the inability to add a new feature and this type of issue is considered technical debt. The last step is about accumulation, evidence of accumulation can be a reoccurrence of the issue, increased time or effort to make implementation change, increased time to find the root cause of a problem. If the problem is not fixed and the effort to maintain a component or part of the system will grow or the cost to fix it later will be significantly greater than fixing it now then the item could be classified as an accumulation [8].

Once the team defines what is not a technical debt, they could start to identify them.

\subsubsection{Technical Debt Prevention}

Several studies have addressed ways to prevent technical debt before it is incurred, and have provided lists of both general and specific strategies that could be implemented in a development project or organization, including architecture decision making support [55], several Agile practices [15], and maintaining a list of bad programming practices [54], among other recommendations. However, the technical debt literature provides general agreement that it is not possible to prevent all technical debt, so the management of technical debt after it has been incurred (the subject of this dissertation) is crucial even if all the recommended prevention strategies are implemented.

According to Eisenberg [26], it costs less to prevent a technical debt than to fix it.

Once the team knows what kinds of technical debt they contracted, then they could define some strategies to prevent the recurrence of the technical debt. Li et al. [55] relates a list with some strategies:

- Development process improvement: to improve the development process to prevent the occurrences of certain types of technical debt [55].

- Architecture decision making support: to analyze potential technical debt caused by different architecture design options and then choose the option with less potential technical debt [55].

- Human factors analysis: to cultivate a culture that minimizes the unintentional technical debt caused by human factors [55]. 
- Life cycle cost planning: system designers can provide solid information related to life cycle costs and then it is possible to make an effective cost plan to minimize the technical debt [90].

Codabux [16] suggested a list of technical debt prevention activities, these activities are:

- Education and Training: if the team members are trained on the organization's development methodology, technology and tools, the probability of implementing the wrong solution can be reduced, also reducing subsequent in rework in design, coding and test phases [16].

- Pair Programming: this practice can reduce unclear, unreadable, duplicated code which is often the source of technical debt [16].

- Test-Driven Development: if the team uses it, they could reduce the risk because the developers better understand the software [16].

- Refactoring: because refactoring improves aspects of code and also productivity [16].

- Continuous Integration: because continuous integration makes the development faster and the team can release a new version at any moment they want. Also, according to them, it can encourage feedback between customers and the development team [16].

- Conformance to process and standards: these activities can contribute to high quality software, thereby reducing the number of defects [16].

- Tools: the tools can help monitor the technical debt contraction [16].

- Customer Feedback: it may help to clarify requirements [16].

- Others: they also suggest other best practices to use in technical debt prevention; review a part of the code base with the objective of identifying defects, have a team that focuses on reducing technical debt, the teams can be dedicated one iteration to reduce technical debt, the teams can plan task with low priority in case they are in trouble it could be dropped and reflective improvement, where the teams can reflect on how to improve their processes [16].

Letouzey [54] suggests that the team could have a list of bad coding practices that create a debt.

Once the teams identified the technical debt items, they can analyze the technical debt types and then define a list of activities, techniques and approaches to implement to mitigate the occurrence of these items.

\subsubsection{Technical Debt Identification}

According to Sterling [95], to be able to start managing technical debt it is important to identify the location, description, and potential cost of not addressing the debt and add it to the Product Backlog for prioritization against future work [95. He suggests that teams and customers should maintain only one (the same) list of work to be done [95].

Bavani [6] suggests that distributed agile teams should track technical debt, including user stories related to pay down the debt in future iterations. He also suggests the automated test to identify and monitor technical debt.

Eisenberg [26] used the Sonar tool to help them to identify some types of technical debt. They selected some metrics and related it to the technical debt list. The metrics were:

- Duplicate code: for them it is a type of technical debt because allows one error to be propagated in the code.

- Rules Compliance: if the code did not follow the standards will be harder to maintain it. 
- Comments-interface: undocumented interface makes the code harder to understand and it can result in an insertion of defects in the code.

- Comments-general: also because a lack of comments makes it hard to understand and maintain the code.

- Package inter-dependence: it would make the code harder to maintain.

- Method/Class complexity: high level of it can be a clue of poor code structure and poor design.

- Automated test coverage: a lack of testing makes it hard to rapidly modify the code and retest.

They also consider the metric manual test coverage, if the code has no automated test. In the Zazworka et al. [110] study they compare four approaches to identifying technical debt: modularity violations, grime buildup, code smells, and automatic static analysis (ASA). In this analysis they conclude that different approaches point to different classes, thus identifying different problems. Also, different approaches provide a view of the software quality at various angles.

After making technical debt visible, it is important to classify the technical debt items according to their type. For this, Guo and Seaman [41] suggest the following types of technical debt items:

- Testing Debt: happened when the team decides to skip tests or reviews in parts of the software, for a particular release or module [41].

- Defect Debt: each time a defect is found but not fixed in that release [41].

- Documentation Debt: when part of software is modified but the documentation is not updated [41.

- Design Debt, technical debt are added when the code has some deficiencies, such as poor programming practices or violations on the system architecture [41].

Li et al. [55], analyzed some studies and identified ten types of technical debt:

- Requirements: is that debt result in a lack between what was defined and implemented as a requirement and what should be implemented.

- Architectural: happen when some architectural decisions compromise some internal quality aspects, such as maintainability.

- Design: correspond to the short cuts that are taken in design.

- Code: refers to an immature and poorly code that violates code rules or/and codes practices.

- Test: refers to shortcuts taken in testing.

- Build: when flaws are made in the process of building, or in the build system.

- Documentation: refers to an incomplete, insufficient and outdated documentation in many parts of the software.

- Infrastructure: relates to a sub-optimal configuration things related to the development, like; process, technologies, tools, etc.

- Versioning: refers for instance to unnecessary code forks, which may imply in problems on source code versioning.

- Defect: refers to defects, bugs or failures found in software systems.

Li et al. [55] gives some examples of each type of technical debt, 2.18 . 
Figure 2.18: Examples of Technical Debt

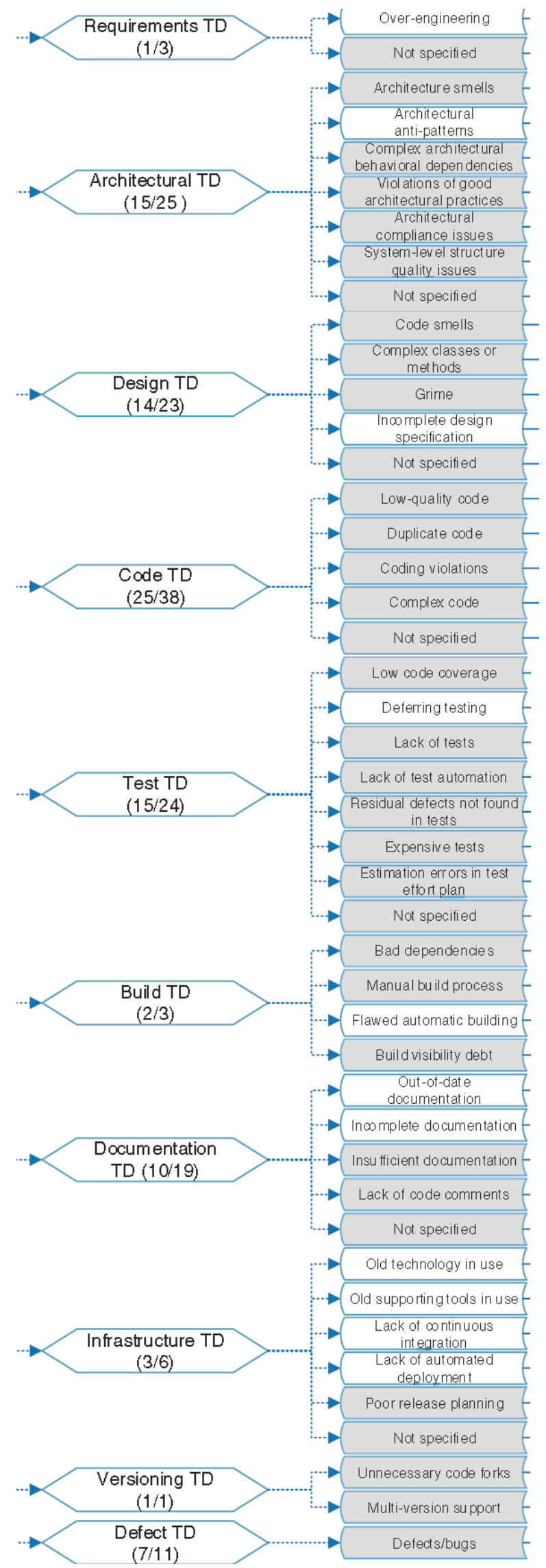

Source: 55]

The list of types of technical debt suggested by Guo and Seaman [41 was used in another 
study of Guo 42, as one of the information items collected at the time of registering a technical debt item. For this, Guo and Seaman [41] used the template given in Table 2.1. [42. Li et al. [55] analyzed 94 studies about technical debt and 54\% (51) of these studies have mentioned technical debt identification as a technical debt management activity. The approaches identified in the studies were categorized by $\mathrm{Li}$ in four types: code analysis ("Analyze source code to identify violations of coding rules, lack of tests; calculate software metrics based on source code to identify design or architecture issues."), dependence analysis ("Analyze dependencies between different types of software elements (e.g., components, modules)"), check list ("Check against a list of predefined scenarios where TD is incurred") and solution comparison ("Compare the actual solution with the optimal solution in some dimension, such as cost/benefit ratio. If the actual solution is not the optimal one, then TD is incurred") [55]. Most of the studies consider the first category code analysis.

Once the software development teams identify the technical debt items, they could define strategies to measure and monitor the technical debt list.

\subsubsection{Technical Debt Measurement}

The measurement of technical debt is difficult because each type of technical debt can be related to many techniques for quantitative characterization [12]. Brown et al. also indicates that it is necessary combine individual measures of technical debt into a good approach to allow a better decision making [12].

According to Guo and Seaman [42], different types of technical debt may require different forms of measures. In addition, we have to select compatible units to measure the different types of technical debt. They suggested using the maintainers experience to estimate the principal, expected interest amount, the interest deviation and the correlations with other debt. According to them, it can be adjusted using historical data or through other types of program analysis, also the defect, usage and change data [42. Guo and Seaman [41] suggests that to measure technical debt it is crucial to measure the following metrics: principal, interest amount and interest probability. He emphasizes that the details of these estimations depend on the granularity of the historical data available. In addition, he said that the interest amount might be very hard to estimate. Thus, he provides a formula to calculate the interest, which is: Interest Amount $\overline{\mathbf{W}} \mathbf{C}$

Where $\mathrm{C}$ is the average cost of the last modifications. The coefficient of $\mathrm{C}$ will be a weighting factor, W, based on the initial rough estimate (in terms of high, medium, or low) of the interest amount [41.

Eisenberg [26] to measure technical debt, they defined the cost associated to remedy each type of debt. In Figure 2.19, we can see the value they defined to each type of technical debt. After that they used Microsoft excel to calculate the cost of technical debt.

Curtis [22] created a function to estimate the cost of technical debt principal, with three variables, the number of should-fix violations in an application, the hours to fix each violation and the cost of the labor. He suggests that to estimate for example, the time to fix the violation could be available from historical effort data. The resulting equation with the three main variables is:

TDprincipal $=((\$$ high-severity violations) $x$ (percentage to be fixed) $x$ (average hours needed to fix) $x$ (U\$\$ per hour $))+((\$$ medium severity violations) $x$ (percentage to be fixed) $x$ (average hours needed to fix) $x$ (\$ per hour $))+((\$$ low-severity violations) $x$ (percentage to be fixed)x (average hours needed to fix) $x$ (\$ per hour)).

They have a specific platform which analyzes an entire application using more than 1,200 rules to detect violations of good architectural and coding practice. The report of this framework aggregates violation scores according to five quality characteristics; robustness, performance efficiency, security, transferability, and changeability [22].

Letouzey [54] suggests creating an estimation model to transform the non-compliance findings into the amount of technical debt. 
Figure 2.19: Cost of Technical Debt by Type

\begin{tabular}{|l|l|}
\hline \multicolumn{2}{|l|}{ Hours to perform, per occurrence: } \\
\hline Remove Duplicate Code Block & 2.0 \\
\hline Automate Uncovered Line/Branch & 0.2 \\
\hline $\begin{array}{l}\text { Manually Test Uncovered } \\
\text { Line/B ranch }\end{array}$ & 0.4 \\
\hline Remove Rule Violation & 0.1 \\
\hline Add Interface Commentary & 0.2 \\
\hline Add Comment Line & 0.1 \\
\hline Remove Package Interdependency & 4.0 \\
\hline Update/Split Complex Method & 0.5 \\
\hline Update/Split Complex File/Class & 8.0 \\
\hline
\end{tabular}

\begin{tabular}{|l|l|}
\hline Cost per labor hour & $\$ 60$ \\
\hline
\end{tabular}

Source: [26]

\subsubsection{Technical Debt Monitoring}

After the technical debt identification the teams should monitor the debts to decided if they have to pay off them or not. Basically most studies suggest using automated forms such as tools and manual forms like defining what level and kind of debt are acceptable for the project or organization.

Guo and Seaman [41] suggest five steps to monitor and make a decision about the technical debt. Initially one needs to identify all technical debt items related to a component that will be modified. Secondly, re-evaluate the estimates for these items based on the current plans. Third, they could do numeric estimates for all items with high interest probability and high interest amount. Then, for each item selected in step 3, they compare cost(principal) with the benefit(interest probability * interest amount) and eliminate any item for which the benefit does not outweigh the cost. Finally, for resulting items, the teams have to decide if the cost can be absorbed in the release. If so, then they can analyzed if any debt can be paid in that release, if not, they can justify the cost to the management and pay the debt [41]. In the second scenario, that it is about verifying if the technical debt is increasing or decreasing, Guo and Seaman [41] suggest using many metrics to analyze the trends, some metrics can be; (1) total numbers of technical debt items, (2) total numbers of highprincipal items, (3) total number of high-interest (probability and amount) items, (4) weighted total principal, which is calculated by adding up over entire list (set 3 points for high, 2 for medium, 1 for low) and (5) weighted total interest (add points for probability and amount) [41].

Another approach is suggested by McConnell [60] to track the rework, to know how much debt is accumulating. Brown recommends creating rules to define the acceptable limits of technical debt [12. In addition, the plugin of SONAR tool can be used to monitor coding rule violations and provide measures using debt heuristics [12. But it is hard to simplify it in simple numbers since it is a complex decision process [12]. Because of this, the management and development environment have to be integrated with monitoring in an appropriate way [12.

Sterling [95] suggests strategically placed runtime exceptions, that can force the developers to think more from the functionality they are working on and what is not involved in that feature. He said that when a developer is currently working they many times find a technical debt. But probably they will not fix it right then because they are working in a different direction, but it will increase the cost of maintenance. Thus, he suggests to create runtime exceptions into the code. This results in some actions where feedback is generated in the development environment instead of in documentation and reports, i.e. at least one test is broken. Also the exception message could provide 
information about the technical debt to facilitate when the developer will deal with it. In addition, it can provide the location of the technical debt. Furthermore, he suggests keeping only one list of work and put the technical debt on the product backlog, where the team and the customer could figure out how the technical debt items can be prioritized 95.

According to Letouzey [54], it is important to define what level and kind of debt are acceptable for the project or organization. Then, monitor the technical debt items over time, frequently so that the team can be able to react fast [54]. When the teams use the SQALE method, it is important that the team define standardized indicators and dashboards and automate as much as possible all the measurement process, training the stakeholders, produce daily reports and define a time to review and maintain the models [54].

\subsubsection{Technical Debt Communication}

With the technical debt vocabulary it is possible to communicate about software quality problems with other people that are affected by the technical debt items. For clarify it is interesting to use a financial vocabulary [60]. Some approaches that can be used are [60]:

- Discuss debt in terms of money rather than in terms of features.

- Continuously discuss the technical debt, instead of just talking about it once.

- Some debt are the result of good business decisions. Others are a result of bad practices or communication, be sure that you taking on the right kind of debt [60].

- Use the budget to service technical debt but, it is important to differentiate the maintenance that keeps the system running and that which extends the capabilities of the system. [60]

The study of Zazworka et al. [111] used the template, Table 2.1 created by Guo [42] to collected information about technical debt items, but he adds one category called Intentional, for this item which indicates if the debt was incurred intentionally or not [111].

In the $\mathrm{Li}$ et al. [55] paper, they suggest a similar template, see Figure 2.5, but with more three items: Context the context of the implementation, for example a programming language and Propagation rules is the impact of the technical debt item in the other parts of the software and Intentionality is whether the technical debt is incurred intentionality or unintentionality [55] [47.

According to McConnell [60], the problem is that the accumulation of the technical debt does not happen visibly, then, he suggests some approaches to identify technical debt:

- Maintain a debt list within the defect tracking system, he suggests that any unresolved debt more than 90 days old have to be treated as a critical debt [60].

- Maintain the debt list as a part of a Scrum backlog, the team could consider each technical debt as a story of the backlog [60]. Furthermore, he proposes to have a schedule to frequently monitor the technical debt items.

Rubin [79] suggests some ways of keeping the technical debt visible, see Figure 2.20 .

As we can observe in the Figure 2.20, in the first approach the author suggests putting the list of technical debt items in that system the team uses to manage their tasks or issues. In the second approach, Rubin [79] proposed that the technical debt items can be a part of the project backlog and to be considered as a new task. He emphasizes that this approach is used when the main objective is to motivate the customer to prioritize them to be paid in the next sprint. Thus, the technical debt item can be estimated and inserted in the schedule.

The last approach to provide technical debt visibility, proposed by Rubin [79], it is to have an exclusive technical debt backlog, it could provide a view of all technical debt items. The author highlights that one simple way to make the technical debt list visible is to put a technical debt board in the team informative workspace.

The studies conducted in this work used TV Screens, graphics from the tools where the technical debt items were registered, discuss in the meetings and boards to communicate on technical debt. 
Figure 2.20: Ways to make the technical debt visible

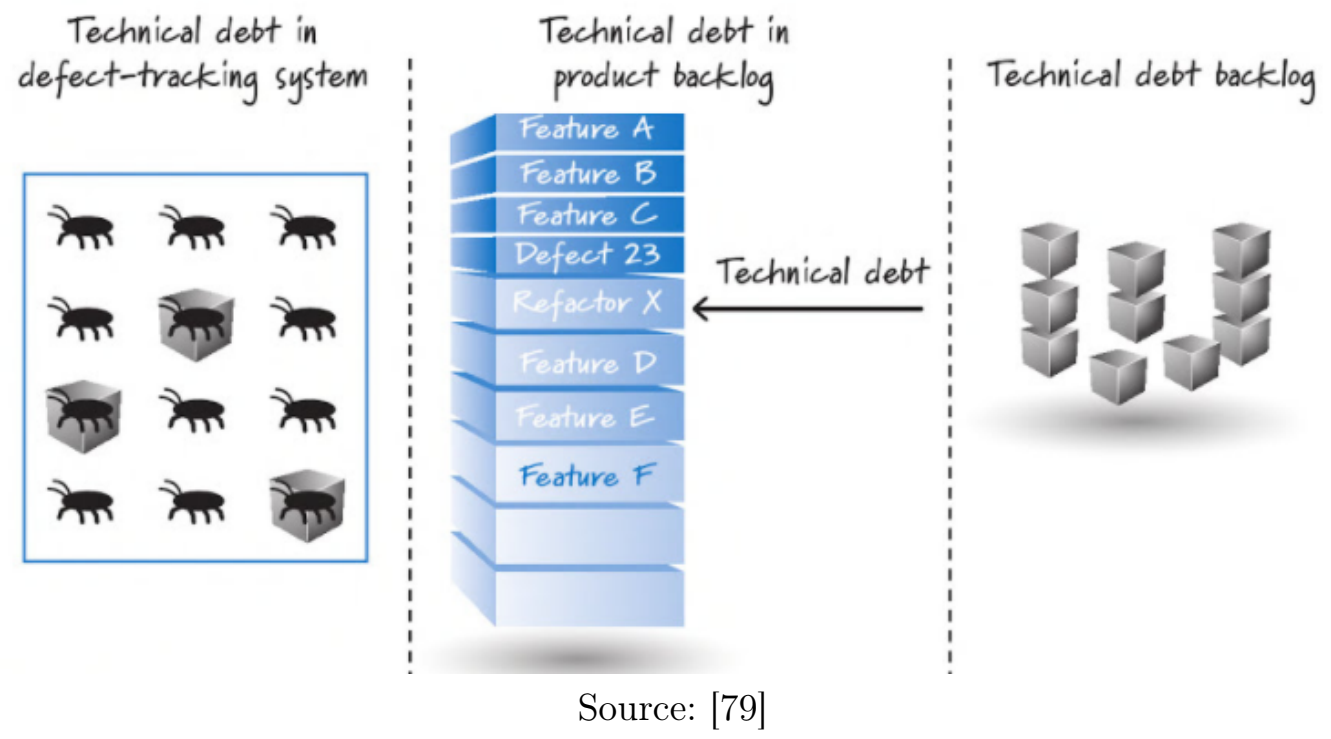

\subsubsection{Tools to Support Technical Debt Management}

In the Eisenberg [26] research, they used the Sonar tool to identify technical debt, but only considered some metrics that were provided in the tool reports, such as; duplicate code, automated test coverage. They started this study just considering the number provided by the technical debt plugin, but once it was so huge, they did not believe that that number reflects the true cost of the technical debt, so they started to consider the metrics related above.

In the Holvitie and Leppanew [47] study, a tool called DebtFlag was created, consisting of a plugin for the Eclipse Integrated Development Environment(IDE) and a separate web application. With the plugin it is possible to capture technical debt items, tracking its propagation and supporting the micromanagement approach. The web application provides a dynamic presentation of the technical debt list [47. The DebtFlag was designed to support technical debt management in two different forms, project and organization level management. This framework is dependent on the technical debt list. Also, the technical debt types are associated with it and the information about their propagation [47]. They define the technical debt interest according to the technical debt propagation. First, the team could create the technical debt types, and then the tool will identify this kind of debt. After that, the teams can see how many technical debt items each element (e.g., method, function) has and how this technical debt propagates in the code [47].

In the Ernest [28] study, they used the RE-KOMBINE tool to manage requirements and to identify requirements problems such as technical debt items based on the notion of optimal solutions. According to them the RE-KOMBINE, can help to make the decision about how much requirements analysis was enough.

According to Li et al. [55], some tools can support technical debt management. He created a list of these tools and categorized them according to what the tools support in relation to the technical debt identification, measurement, prioritization, monitoring, prevention, communication, repayment and representation/documentation. They also categorized tools according to the artifacts the tools support to analyze, such as code. Also, which type of technical debt it is possible to identify with the tool. This study is a recent study with a large list of tools that could help software development teams to manage technical debt. Most of these tools are related to static code analysis metrics. However, the tools, Sonar Qube, DebtFlag and the technical debt Evaluation SQALE plugin for Sonar Qube are tools that specifically deal with technical debt items.

Sanchez et al. [32] created the TEDMA tool. The tool can be used for technical debt monitoring over the software evolution. This tool analyzes the source code repositories considering changes in files over the software evolution. Thus, the tool creates a graph database with the role analysis of 
the software evolution. This tool has available some data analyzers that are PMD and Findbugs. Also the tool had directly implemented the analyzes of other metrics, for instance, the probability of change and expected size of change as are defined in.

\subsubsection{Technical Debt Prioritization and Decision Making}

According to Guo and Seaman [41, the goal of identifying and measuring technical debt is to facilitate decision making. They listed two scenarios in which technical debt information can be used in making a decision; the first is part of release planning and the second is ongoing monitoring of technical debt over time, independent of the release cycle [41].

When a team decides to contract a debt or not, they commonly have to decide between two ways, one is faster and other results in better quality [60]. If the team chooses the faster way, in most cases, they will pay more to do it, because the cost is the sum of the time they used to implement fast, plus the time they used to correct it, plus the cost resulting for making this decision [60]. The other variable that is important to reflect is how slowly the team will continuously develop because of the existing debt [60]. Another thing to consider is if there is a better solution as fast as the fast option but that does not affect the rest of system [60].

In the Seaman et al. [88] study they discuss some types of decision making to apply in a technical debt data aiming to manage technical debt.

Einsenberg [26] defined a list of metrics to identify technical debt, then they make a rank of each technical debt is more critical linked with each kind of metrics were more critical in a specific moment of the project, according to a set of characteristics of the software development. These characteristics were:

- Development Methodology: test automation, minimization of complexity and package may be more important when employing agile methodologies.

- Failure Criticality: it could result in thresholds that are more stringent across the board, with particular emphasis on test coverage.

- Security Requirements: secure code requirements need stricter rules, then it is important to emphasize things like test coverage and rules compliance.

- Life cycle Phase: in the moment of the life cycle it is a development or maintenance or legacy code can influence the priorities.

- Planned Reuse: if the team intend to reuse some parts of the code in the future, can be priority preventing duplication and package tangle.

- Requirements: if it is some contractual obligations, for example, rules compliance, it has to be a priority.

- Project Status: for example, if the defects average is high, it may influence priorities [26]

After that, they did a board with green, yellow and red status, to identify what technical debt items were more important than other. This rigorous was defined by the program characteristics and priorities. An example of this can be seen in the Figure 2.21.

A SIG/TUViT's software quality assessment method was used to calculate technical debt in the Nugroho et al. 68 study. According to them, this method was developed as a layered model for measuring and rating the technical quality of a software system in terms of the quality characteristics of ISO/IEC 9126 [68]. In the first step, the code is analyzed to collect information such as; code duplication, cyclomatic complexity, dependency counts, parameter counts, lines of code. After that, metrics are organized into ratings for properties at the level of the entire software product, such as volume, duplication and unit complexity. Then, a rating was made using the values in the interval between 0.5 and 5.5. The aim of this scale is to facilitate communication and comparison of quality results at the level of entire software products. Likewise, they define a mapping of risk profiles 
Figure 2.21: List of Technical Debt Priorities

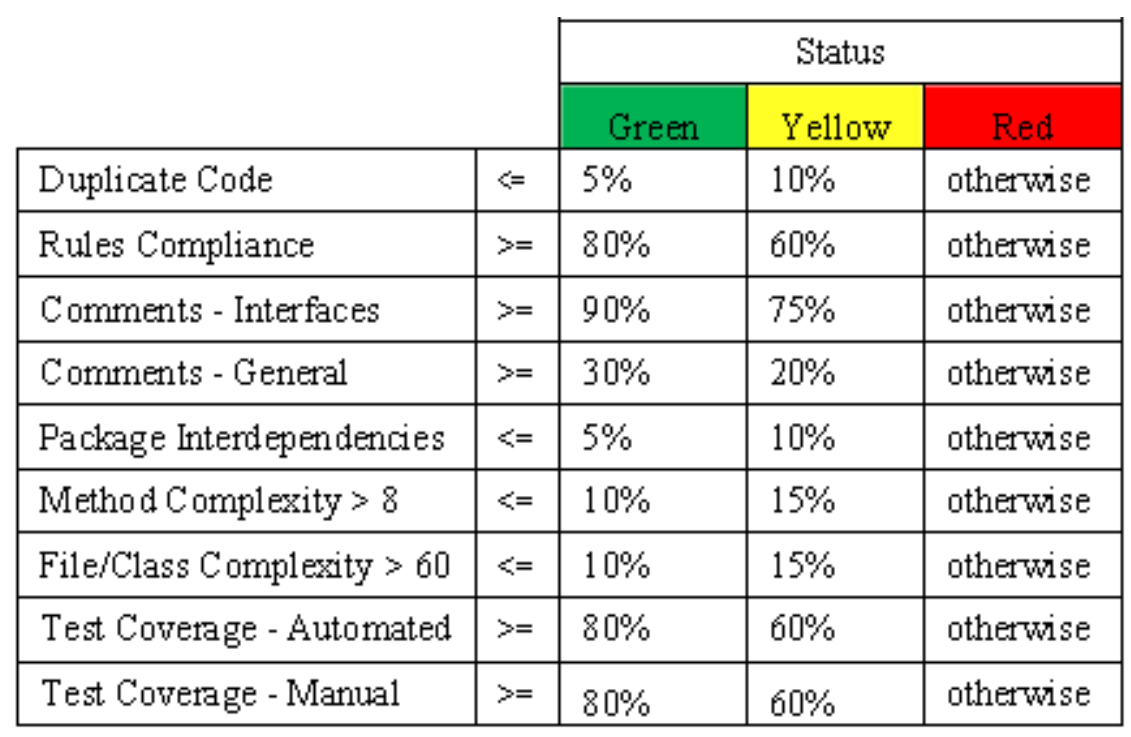

Source: [26]

to quality ratings. They used the Software Analysis Toolkit (SAT) of SIG for the extraction of measurement values from source code. In the end, all sub-characteristic ratings are averaged to provide the overall maintainability rating [68].

The SQALE method was created by Letouzey [54] to measure and to manage technical debt. This method is based on nine principles. First, the Company or team has to create a Quality Model, that is the list of nonfunctional requirements that are considered "right" code. This quality model is used as a reference to calculate the technical debt. The SQALE method also requires that the teams develop an estimated technical debt model, for this they could associate each requirement with a remediation function. These requirement can be things about maintainability - There is no commented out block of instruction, testability - all files have unit testing with at least $70 \%$ code coverage, reliability, changeability, architectural and others [54]. Therefore, the technical debt will be the sum of remediation costs for all noncompliance [54. After that, according to him, the SQALE method defines additional indexes and indicators to analyze and understand the debt, as we can see in Figure2.22, [54].

In this case the characteristic testability is the first characteristic that the team needs and each requirement is connected to a characteristic. Therefore, they provide a graphic with the distribution of the technical debt items for each of the selected characteristics [54]. The method also requires associating the requirements to a non-associate function. It is used to quantify all costs of the delivery of one or more non-conformities [54]. Then, the non-remediation function estimates the penalty the business will have to accept as compensation for accepting violations. SQALE also has an index to sum all the non-remediation costs associated with a given scope, they call it the business impact index 54 .

\subsubsection{Technical Debt Payment}

Once the teams monitor, measure and manage technical debt, in a specific moment they have to decide whether pay of the debt or not. However, the Allman [1] study emphasizes that technical debt is almost never paid off in its entirety. According to him, most systems, during their lifecycle, have items to fix or to do. And the cost of technical debt will be paid by refactoring or code rewrite [1]. If the interest is less than the cost to pay back the technical debt, the debt does not have be paid [1]. According to Guo and Seaman [42, the teams have to decide if the technical debt item continues to be a benefit or if it is better to it pay now. For this, they need to determine what the net benefit of the item is, calculating the principal minus the expected interest amount. Also, 
Figure 2.22: The organization of "right code" requirements in a chronological order.

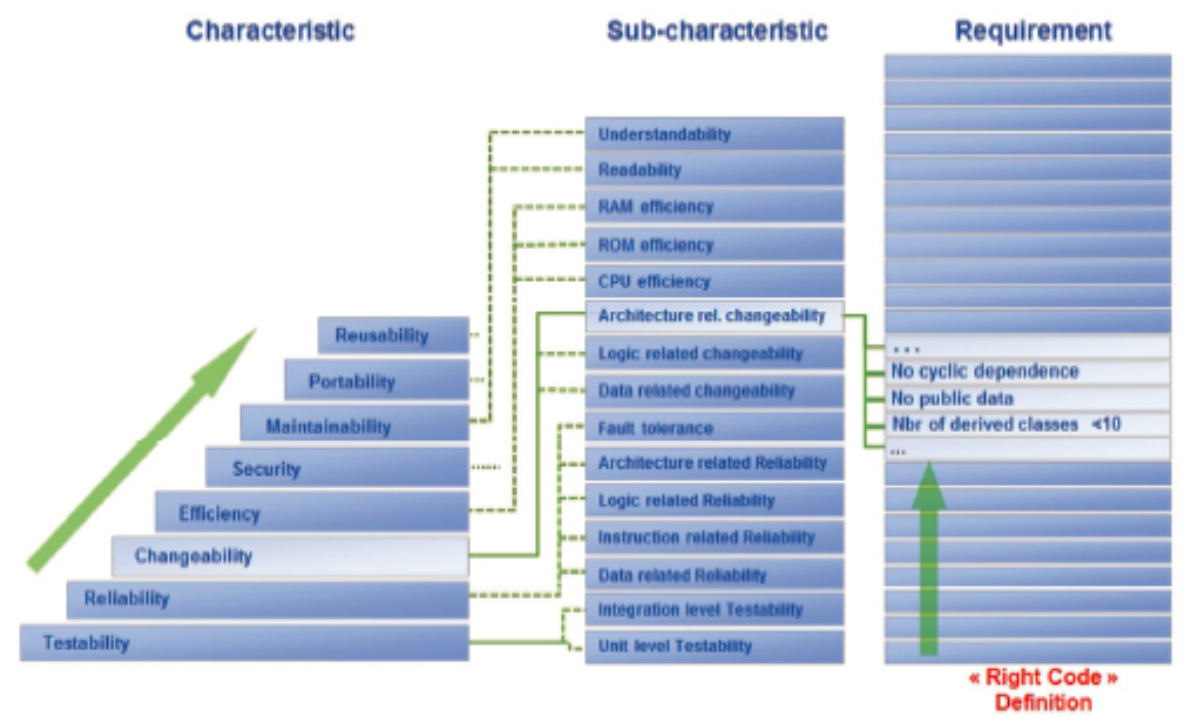

Source: [54]

according to him, the teams have to balance the expected net benefit with the risk of the item not producing a benefit (for instance, if the model affected will not be modified), this measure is the interest standard deviation [42]. According to Sterling [95], while the technical debt is not paid, more code will surround and interact with the debt, thus it will be more difficult to remove the debt and the cost of maintenance increases. He suggests paying off technical debt immediately because if the team decides to pay later, then commonly this later never arrives.

Allman [1] suggests that the strategic part of the technical debt items have to be paid promptly; the farther it goes, the more difficult to change, because people go to other companies, aggregate new compromises, move to other projects, etc [1].

The more the amount of technical debt the more the software progress will slow down [60. The study of McConnel [60] indicates several reasons to pay technical debt. The software development team can be able to deploy new release faster. Also, they could add some functionality in specific areas, that at that moment is impossible because of the debt. If they add a lot of debt in a specific moment it can be more visible to the customer and to result in a many errors in the system [60.

A key factor to pay off technical debt is related to the amount of un-intentional technical debt are contracted by the team; the less they contracted this kind of debt, the more they can use technical debt as a strategy [60. McConnell [60] proposes that after the debt has been incurred, in the first iteration, teams should be devoted to pay it. They also highlighted that the decision of pay off technical debt also depends on the kind of system the team develops and the impact to deploy a new release [60].

In Holvitie and Leppanew's [47] work they concluded that some factors and criteria that can be used to make the decision to pay or not pay a technical debt is heavily influenced by the accumulation of technical debt in the deferred defect form. Because of this, they created a list of some criteria that can help the teams to make the decision;

- Severity: the severity explores the capabilities that are affected by the defect that will concern the customer [47].

- Existence of a workaround: an alternative solution, can make it possible to defer the fix to a later version [47].

- Urgency of fix required by customer: sometimes the customer can request that a particular defect has to be fixed urgently, then it has to predate the other tasks [47. 
- Effort to implement the fix: the developer estimates the effort and time required to implement and test the defect, the test team estimates the effort and time to validate the fix 47]

- Risk of the proposed fix: the developer considers the affected software components, functions and classes to evaluate the risk of the solution [47].

- Scope of testing required: the team considers the impacted requirements and functions to determine the scope of selected test cases and whether system regression needs to be run on the resulting fix. They have to consider also the impact of the fix to nonfunctional requirements in such areas as performance, scalability, usability, and maintainability [47].

Letouzey [54 suggests having a technical debt list and then monitoring and repaying it. If the debt breaks the established limits, the team has to fix them to return to acceptable limits. To make this decision the team can analyze variables, such as deadline, budget and impact. He also suggests analyzing the debt in correlation with other variables such as business value or quality perceived by users.

In our studies the teams used tools, such as SonarQube, and manual ways to monitor technical debt, for instance, discussing the list of technical debt during the Sprint Planning meetings.

\subsection{Chapter Summary}

This chapter described the technical debt genesis and the main concepts of this area. We explained some studies about the technical debt metaphor, main characteristics and the technical debt properties, based on a literature review. These studies are essential to software development teams understand the technical debt metaphor, so they can start to identify the debts. We discussed some risks that the teams assume when they incur debt, as well as the impact of contracting it. Furthermore, we show the models of technical debt management found in the literature review. These models will be compared with our model in Chapter 7 . We also give some examples of non-technical debt, it can help the teams to define what they will consider as technical debt in their projects. Moreover, we explain about technical debt prevention and we describe the important aspects of this area. In addition, we discuss techniques, tools, and forms to identify and monitor technical debt. We present several studies relating some approaches for technical debt measurement, prioritization and decision-making. Some tools that teams can use to identify and monitor technical debt are described. Challenges in each of these phases of technical debt management are discussed in the previous sections. 


\section{Chapter 3}

\section{Research Method and Design}

This chapter presents the research methods and the techniques of data collection and analysis that were used to conduct the research. However how these methods were applied will be reported in Chapters 4, 5 and 6, which describe the methodology and results of each study. In addition, this chapter explains the steps in the overall design of this research.

\subsection{Research Method and Design}

In this study, we conduct a qualitative research. According to Merriam [64, "...qualitative research attempts to understand and make sense of phenomena from the participant's perspective. The researcher can approach the phenomenon from an interpretive, critical, or postmodern stance. All qualitative research is characterized by the search for meaning and understanding...". This is directly connected with the purpose of this study and with the characteristics of the cases studied. Given that the main goal of this research was to create a model to identify, monitor, and manage technical debt as well as to explore the impact on the agile teams once they become aware of the technical debt concept. As the second characteristic of qualitative research, Merriam also highlights [63] the fact that the researcher is the primary instrument for data collection and data analysis. In each study, the planning, the definition of which data would be collected, and the final result of the data analysis were discussed with both the advisor and the co-advisor. The studies in the Extreme Programming Laboratory and at Company A were analyzed by two other researchers as well.

The strategies we applied in this research were case studies and action research. The qualitative research process is inductive [63] and the researcher collects and analyzes data to build concepts, hypotheses or theory. Therefore, the data was collected through interviews, observations, document analysis, tool reports, and questionnaires. The data analysis was done qualitatively [50], using the coding steps of the Grounded Theory approach.

It is explained below in which case each method was applied, as well as the motivations that led us to choose such methods.

\subsubsection{Case Study}

According to Yin [107], a case study is an empirical investigation that analyzes a phenomenon in a real context. Robson and Runeson [78, 80] affirmed that this type of study is used to discover what is happening and to explore new ideas.

Aligned with the main objective of this study we also carried out an exploratory case study. Our goal was to understand the phenomena the interpretation of their context [33].

According to Yin [107], it is important to define five components in the case study design : the study's questions, the study proposition, the unit of analysis, the logic linking the data to the propositions, and the criteria for interpreting the findings.

- The study question: defining a research question is the most crucial step of the study [107]. According to Yin [107], the choice of the type of method depends on the type of question to 
be answered, and according to the author, there are 5 types: "Who", "What", "Where", "How", and "Why". This study sought to answer the "What" and "How" questions.

- The study proposition: the proposition is something that would be analyzed in the study scope. But in an exploratory study, we can state a purpose rather than a proposition [107].

- The unit of analysis: we have to select a case to be studied. Easterbrook et al. [25] shows that one of the most crucial steps in the case study is the selection of cases. According to Merriam [63], it is important to select cases that are more relevant to the study proposition. A simple case study can be enough, but a multiple case design could result in greater validity [63]. According to Merriam [63], the unit of analysis might be a project, a team, a company, an individual developer, etc. We select as the unit of analysis a team, since our questions focused on understanding how teams could manage technical debt in their projects and what was its impact.

- The logic linking the data to the propositions and The criteria for interpreting the findings: these two components are related to the data analysis steps. One way to do this is to relate pieces of information from the same case to the purpose [107]. In this study, we did it by creating codes, following the coding steps of Grounded Theory and relating them to the research questions.

Different types of data source were collected following the [63] recommendation so it could offer rich insights into the case.

We can analyze the validity of the case study considering four criteria [107:

- Construct validity: defining appropriate operational measures for data collection. For instance, using multiple sources of evidence, establishing a chain of evidence, having key information, and reviewing draft case study report.

- Internal validity: For instance, doing pattern-matching, do explanation-building, and do timeseries analysis. According to Yin [107], it is a concern only for explanatory or causal case studies. It occurs if the research concludes that there is a causal relationship between $\mathrm{X}$ and $\mathrm{Y}$ without considering that some third factor $\mathrm{Z}$ also has some influence on it. For example, using replication.

- External validity: establishing the domain for generalization.

- Reliability: showing that the operation of the study can be repeated with the same results such as data collection procedures [107]. For example, by developing a case studies database and using case study protocol.

The case study method was applied in the analysis of the cases studied in Company A (the fictitious name created due to confidentiality issues) and also in the studies we did within the classroom environment. In these cases, semi-structured interviews, participant observation, and questionnaires were used for data collection, all of them composed of open and closed questions. We discuss the validity threats in each case in the next chapters.

Merriam [63] emphasizes that the major weakness of case studies is the bias the researcher can input in the interpretation of the data collection and analysis. Hence, it is crucial to develop an explicit framework to select the cases and the data collection [63].

\subsubsection{Action Research}

Action research is a type of empirically based social research, where researchers and participants are involved cooperatively, seeking to implement certain actions to solve certain problems [58].

According to Baskerville [5], action research enables us to better understand complex social processes by inserting changes in these processes and observing the effects of such changes. The 
action researcher is concerned creating organizational change and simultaneously on studying the process [5]. The main objective is to intervene in the study situation in order to improve something there [25]. The action research cycle is divided into five steps, as we can see in Figure 3.1.

Figure 3.1: Action Research Cycle

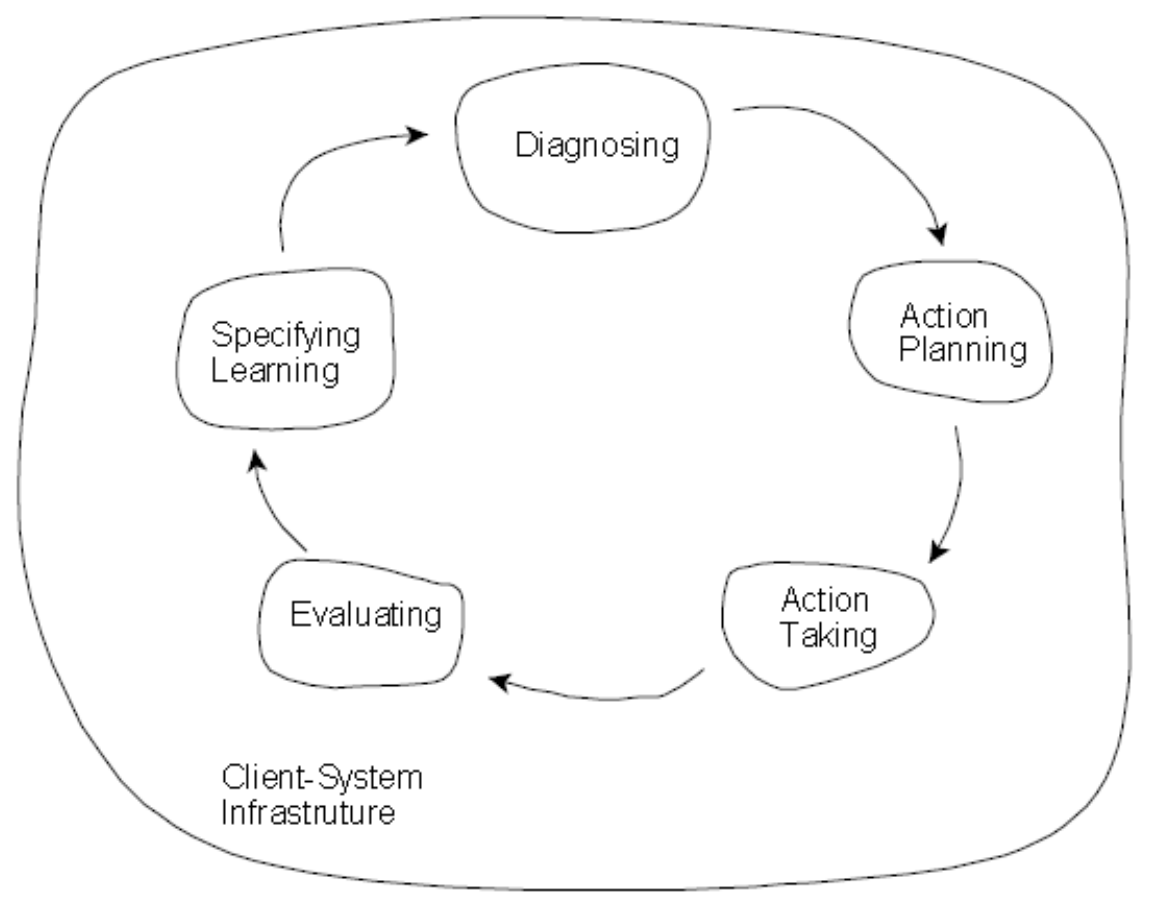

Source: Baskerville [5].

The Client-System Infrastructure constitutes the research environment [5]. The activity of Diagnosing stage corresponds to the identification of the main problems that are the underlying causes of the organization's motivation to implement the changes. Diagnosing involves selfinterpretation of the complex organizational problem [5]. Once the problems have been identified, researcher and professionals collaborate with the Action Planing, this stage defines the changes that will be implemented to reach a certain objective (relieve or improve the problem that was identified in step one). Action Taking is the third stage, when researchers and practitioners implement the planned action. If necessary, they can carry out interventions so that the actions are implemented. Evaluating is performed after the plan is implemented, by analyzing the outcomes and determining whether the theoretical effects of the implemented actions have contributed to solving the problem. If not, the plan should be adjusted for a next iteration. If so, a critical evaluation should be done to identify whether the implemented actions were the only motivating causes of success. And in the last stage, Specifying Learning, the evaluation is carried out in order to identify if the acquired knowledge motivated the implementation of some organizational changes. The knowledge acquired in this cycle should direct adjustments for new interventions in the next steps of action research [5].

The action research method was applied in the study carried out in Company B (the fictitious name created due to confidentiality issues).

\subsection{Methods and Techniques of Data Collection and Analysis}

When we conduct studies on real-world issues, there is a compromise between the level of control and the degree of realism. Given that the real situation is often complex and not deterministic, it is difficult to understand what is happening, particularly for exploratory purposes [25].

The data collected in the research may be qualitative or quantitative. Quantitative data involve 
numbers, while qualitative ones involve words, descriptions, photos, diagrams, etc. The quantitative analysis is done through statistics and qualitative analysis is done through categorization and classification [86]. Another important factor to increase the precision, validity, and reliability of empirical research is to use data triangulation, which means to analyze the object studied from different angles, using different data sources, thus providing a better view of the case studied and contributing to increasing the degree of reliability of the findings [25].

Three different types of triangulation can be applied [94]:

- Data Triangulation: using more than one data source or collecting data at different times [94].

- Methodological Triangulation: combining different types of data collection methods, e.g. qualitative and quantitative methods [94].

- Triangulation and Theory: using alternative theories or points of view [94].

In this research we used data triangulation when the data was collected using the following techniques [86]:

- Interview: is a qualitative data collection technique. It can be used to achieve a variety of objectives: to collect historical information from the interviewees' memory; to collect opinions or impressions about certain events; to understand certain terminologies in a specific field. Herein, semi-structured interviews were conducted, which are those formed by open and closed questions. The interviews were mainly used to gather opinions on forms, impact, and relevance in the identification, monitoring, and management of technical debt and to identify the impact on the team once they were aware of the technical debt.

- Questionnaire with open and specific questions: were used to identify the perception of the team regarding the object of study according to factors such as forms, impact, and relevance in the identification, monitoring, and management of technical debt. It was also used to identify the impact on the team once they were aware of technical debt.

- Documents: data were collected from documents, emails, reports from code quality analysis tools, project management tools, and graphs designed specifically to monitor technical debt metrics.

- Participant Observation: we also used the direct observation approach, which according to Yin [107], is the one where the participant visits the study site and collects evidence about the context and the phenomenon being studied. According to Seaman et al. [86] "...Although the name is misleading, participant observation does not necessarily imply that the observer is engaged in the activity being observed, only that the observer is visibly present and is collecting data with the knowledge of those being observed...".

\subsubsection{Method of Data Analysis}

The steps of the Grounded Theory approach were followed for data analysis. The Grounded Theory approach was created by Glaser and Strauss [38] in the social sciences with the aim of constructing theory through an inductive and systematic analysis of data collected [38]. Ergo, learning must emerge from the data rather than from an existing theoretical view. An important feature of this approach is the method of constant comparative analysis, where the researcher analyzes the data several times [38]. To reduce bias, it is recommended that more than one researcher analyze the data. The Grounded Theory consists of three basic elements: concepts, categories and properties. There are two distinct approaches to apply Grounded Theory. One is defended by Strauss and Corbin and the other by Glaser. In this work, we are following the approach suggested by Strauss [96]. This approach is based on the idea of coding, which is the process of analyzing data and transforming small parts into conceptual codes, then grouping them into categories until they emerge in a single final category that represents them, aiming at creating a theory [18]. This technique is divided into three parts [97, 96, 19]: 
- Open coding: it is an interpretive process, where the collected data are analyzed line by line, events/actions/iterations are broken down and compared with similar or different codes previously created, thus creating conceptual codes. Codes with similar concepts are grouped into categories and subcategories [18, 19, 97, 96].

- Axial coding: the categories and subcategories are related and once again these relations are analyzed and tested in comparison to the data [18, 19, 97, 96].

- Selective coding: in selective coding, every process is refined by identifying a core category of the theory. This category may be an existing category that has emerged for this or may be a new category that expresses the essence and/or represents the other categories [18, 19, 97, 96].

It is important to highlight that we have used the Grounded Theory approach not with the objective of creating a theory, but with the objective of following greater scientific rigor in the analysis and interpretation of the results.

In Figure 3.2, we can see the research timeline. We started the research in 2012 and finished the data analysis in 2016. In Figure 3.2 we show when and where each study was carried out and what questions guided that study. 
Figure 3.2: Research Timeline

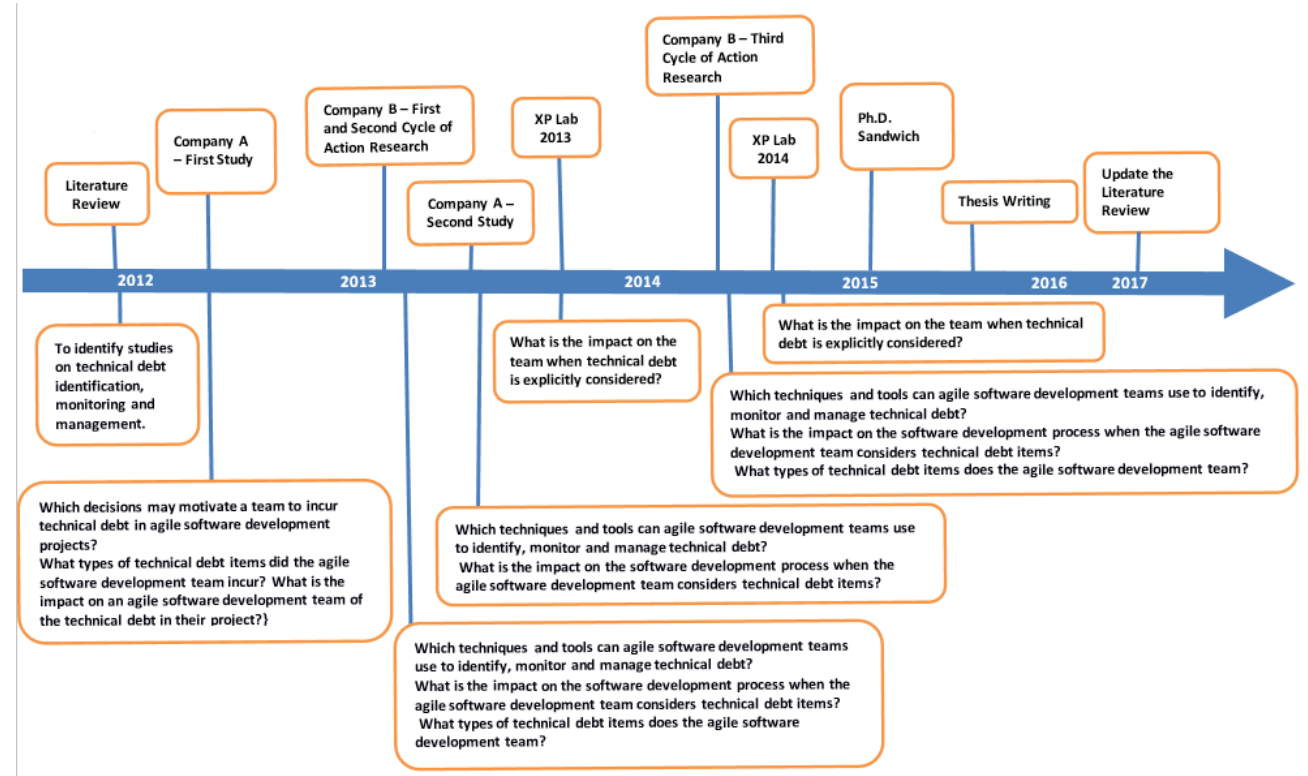

In the next section, we describe each step of the research related to Figure 3.2 .

\subsection{Research Steps Overview}

This research was developed in five main steps, as can be seen in Figure 3.3 .

The main goal in Step 01 was to select studies related to the identification, monitoring, and management of technical debt. At the same time, the Ph.D. proposal was written. Since there there were not many studies on the subject, in 2012, it was decided to consider all the works in the area of technical debt. However, in the last five years (2013, 2014, 2015, 2016, 2017 and 2018) many works were published in the area and therefore, only those directly related to this research came to be considered.

In Step 02, we started to contact an Information Technology Company, which for this study we will call Company A. In the first meeting, the research study was presented to the R\&D director, who suggested some projects to be investigated. Then, a study was conducted in four company projects. Firstly, we did a team diagnosis trying to identify whether the team members responsible for the projects were familiar with the concept of technical debt. After this diagnosis, a conversation and explanation about technical debt were conducted with the teams. The matter was subsequently discussed in order to verify if the teams believed that the projects in which they worked presented any technical debt items. Next, based on the results found in Tonin et al. and Gomes et al. [102, 40] master's research, we sought to identify moments in time when major changes in the projects were requested and to verify if these changes resulted in the occurrence technical debt items. After that, we identified some technical debt items and investigated if they originated from strategic decisions. A model was created to show possible causes for the incurrence of these technical debt items. Furthermore, we began an action research study in a Software Development Company, Company B. The goal was to suggest ways to identify, monitor and manage technical debt. We used the techniques and forms identified in the literature review up to that point, including those that had been tested in previous case studies performed. Firstly, we explain the study to the company manager. After that, we made a presentation on technical debt to two of the company's software development teams. Along with the team members, we planned how to start the first cycle of action research. In this cycle, the aim was to start identifying and monitoring technical debt.

In Step 03 a study was carried out with students of the Extreme Programming Laboratory course (2013 edition) of the Institute of Mathematics, Statistics and Computer Science of University of São Paulo. In this study, a conversation was held with the students to discuss the concept of technical 
Figure 3.3: Research Steps

\begin{tabular}{|c|c|c|c|c|}
\hline & Study Name & Setting & $\begin{array}{c}\text { Research } \\
\text { Method } \\
\end{array}$ & Main Objective \\
\hline Step 01 & $\begin{array}{l}\text { Literature } \\
\text { Review }\end{array}$ & Academic & $\begin{array}{l}\text { Literature } \\
\text { Review }\end{array}$ & $\begin{array}{l}\text { To identify studies on technical } \\
\text { debt }\end{array}$ \\
\hline \multirow{2}{*}{ Step 02} & $\begin{array}{l}\text { Company A - } \\
\text { First Study }\end{array}$ & Company & Case Study & $\begin{array}{l}\text { To identify the impact of some } \\
\text { technical debt }\end{array}$ \\
\hline & Company B & Company & $\begin{array}{l}\text { Action } \\
\text { Research }\end{array}$ & $\begin{array}{l}\text { First Cycle - Start identifying } \\
\text { and monitoring technical debt }\end{array}$ \\
\hline \multirow{3}{*}{ Step 03} & Company B & Company & $\begin{array}{l}\text { Action } \\
\text { Research }\end{array}$ & $\begin{array}{l}\text { Second Cycle - To improve the } \\
\text { ways of identifying and } \\
\text { monitoring technical debt }\end{array}$ \\
\hline & $\begin{array}{l}\text { Company A - } \\
\text { Second Study }\end{array}$ & Company & Case Study & $\begin{array}{l}\text { Start identifying, monitoring } \\
\text { and management technical debt } \\
\text { without interfering in the } \\
\text { software development process } \\
\text { of the agile team }\end{array}$ \\
\hline & XP Lab 2013 & Academic & Case Study & $\begin{array}{l}\text { First analysis of the impact on } \\
\text { the team after considering } \\
\text { technical debt }\end{array}$ \\
\hline \multirow[b]{2}{*}{ Step 04} & Company B & Company & $\begin{array}{l}\text { Action } \\
\text { Research }\end{array}$ & $\begin{array}{l}\text { Third Cycle - Start to manage } \\
\text { technical debt }\end{array}$ \\
\hline & XP Lab 2014 & Academic & Case Study & $\begin{array}{l}\text { Second analysis of the impact } \\
\text { on the team after considering } \\
\text { technical debt }\end{array}$ \\
\hline \multirow{6}{*}{ Step 05} & PhD sandwich & Academic & - & Discussion of the data collected \\
\hline & Data Analysis & Academic & - & Analysis of the data \\
\hline & $\begin{array}{l}\text { Thesis Writing } \\
\text { in Portuguese }\end{array}$ & Academic & - & $\begin{array}{l}\text { Start writing the thesis in } \\
\text { Portuguese }\end{array}$ \\
\hline & $\begin{array}{l}\text { Thesis Writing } \\
\text { in English }\end{array}$ & Academic & - & $\begin{array}{l}\text { After the Dagstuhl Seminar, } \\
\text { translate the thesis into English }\end{array}$ \\
\hline & Paper Writing & Academic & - & $\begin{array}{l}\text { Publish a paper at the } \mathrm{XP} \\
\text { Conference }\end{array}$ \\
\hline & $\begin{array}{l}\text { Update the } \\
\text { Literature } \\
\text { Review }\end{array}$ & Academic & $\begin{array}{l}\text { Literature } \\
\text { Review }\end{array}$ & $\begin{array}{l}\text { Reading and analyzing recent } \\
\text { papers }\end{array}$ \\
\hline
\end{tabular}

debt. After this conversation, forms of identification and monitoring of technical debt items were suggested and the teams started using them. At the end of the study and according to the team members' perception, it was sought to identify impacts on the team and the project after considering technical debt. To do this, we used the tools already used by the teams in their projects.

Also as part of Step 03, another study was carried out at Company A with the objective of suggesting a prototype model for the identification, monitoring, and management of technical debt, trying not to cause big interference in the team process, despite using its tools. This study is also part of a master's degree dissertation [73], although the study strategies, the study interviews, and the analysis of the data were done by both the researcher of this thesis and the researcher of the master's degree dissertation.

We also started the second cycle of action research at Company B. The aim was to improve the ways to identify and monitor technical debt items.

In Step 04, another study was carried out at the Extreme Programming Laboratory course (2014 edition). In this study, the goal was to identify the impact of considering technical debt on both the team and project, according to the perception of the team members.

The third cycle of action research was then carried out at Company B. The main objectives were to improve the previously used ways to identify and monitor technical debt and to start managing technical debt. The studies in this company were finalized because some key people for the research left the Company.

In Step 05, we did a Ph.D. sandwich in the University of Maryland Baltimore County(UMBC) with the professor Ph.D. Carolyn Seaman. During this time, we discussed the analysis of the data. During this step, we also finished the analysis of the data, wrote the thesis, and submitted some papers. In addition, we updated the literature review and found several new studies. 
In the next chapters, we present in detail how each study was conducted and what were the results.

\subsection{Chapter Summary}

This research is composed of a variety of studies that use different methods and are in different contexts in order to triangulate the findings. This chapter gives an overview of the research approach and how the different studies are conducted fit together. Also, in this chapter is described, in general, the techniques we used to collect and analyze data. It also presents the main reasons that led us to choose such methods and techniques. Finally, we explain the research steps. Details of the research methodologies of each study are provided in chapters 4,5 and 6 . 


\section{Chapter 4}

\section{Classroom Studies}

Two studies were carried out in the Extreme Programming Laboratory Course at the University of São Paulo, one in the 2013 offering of the course and the other in the 2014 offering. We will discuss below how the studies were developed in each case, as well as the applied research design and the results obtained.

In this chapter, we discuss the context of the studies, present the approaches we used to conduct the research and how we applied them. We explain the main findings and summarize the results of our research. Then, we discuss the threats to validity.

\subsection{The Extreme Programming Course}

The Extreme Programming Laboratory (XP Lab) Course 39] is offered annually for undergraduate and graduate students in the Computer Science department in the Institute of Mathematics, Statistics and Computer Science at the University of São Paulo. The motivation is to provide the students an opportunity to learn agile software development methods on real projects [82]. Customers submit proposals of possible projects to be developed during the course. The selected projects are developed by the students following the principles and values of the agile method Extreme Programming (XP). Extreme Programming emphasizes teamwork - managers, customers and developers are all equal partners in a collaborative team. The main values of Extreme Programming are communication, simplicity, feedback, respect, and courage [7]. The objective of our research is to characterize the impact on the team when technical debt items are visible, based on team members' perceptions. This study aims to answer the following research question (RQ):

- RQ9: What is the impact on the team when technical debt is explicitly considered?

The study was applied in two offerings of XP Lab. Four teams (with five or six students each) were followed in the 2013 offering and five teams (with six students each) in the 2014 offering. We conducted the study and collected data through questionnaires and interviews, and analyzed the source code of the projects with Sonar Qube and Code Climate.

XP Lab students count on the support of meta-coaches who are experts in agile methods. They provide agile mentoring for all the teams with the professor's help. Each team also has a coach who is a student who has more experience in agile methods - there were cases where a team had two coaches, since more than one student had expressed the willingness to take on this role. The teams develop real projects with on-site customers. The teams have to follow some agile practices, such as; pair programming, automated tests, continuous improvement, continuous integration, etc. In both studies, the teams worked in pairs and in threes, and the groupings changed many times during the course, sometimes according to the tasks they needed to develop. The course requires a minimum attendance of at least 8 hours per week (four hours in the laboratory and four hours of extra classes) and there is a lunch meeting once a week to encourage the students' presence in the lab and to allow the students an opportunity to share their experiences. On some weeks, there are 
short presentations about any difficulties that the teams are facing, where a specialist explains and discusses specific topics. A complete description of the course settings can be found in 82 .

The course provided physical and technological infrastructure for learning XP in a context very similar to the business context 82 . The teams were divided into two laboratories where each team had their own physical environment and collaborative workspace [69]. The teams used the Planning Poker technique to estimate the tasks and if they found appropriate, they could hold their retrospective meetings in other environments rather than inside the laboratory where they worked.

In the first class, the teacher presented the objectives of the course, structure, programming, evaluation criteria, and available projects. Each student chose three projects that they were interested in participating in. At this stage, the coaches were also chosen [82]. In the second and third day of the class, the XP method was explained to students who would act on the team as developers.

Separately, meta-coaches taught coaches how to run agile projects with XP. Next, the students were introduced to their respective workspace environment, projects, customers, and coaches [82].

\subsubsection{Projects}

In Table 4.1. we briefly describe each of the projects involved in our study.

Table 4.1: Extreme programming projects.

\begin{tabular}{|c|c|}
\hline Project & Description \\
\hline Arquigrafia & $\begin{array}{l}\text { Arquigrafia is a nonprofit public collaborative environ- } \\
\text { ment dedicated to the dissemination of architectural im- } \\
\text { ages, with particular attention to Brazilian architecture } \\
{[3] \text {. }}\end{array}$ \\
\hline Games-VidaGeek & $\begin{array}{l}\text { A platform for games that supports the teaching of pro- } \\
\text { gramming (with games for Scala, Java, Html, CSS, SQL, } \\
\text { and other languages under current production) [106]. }\end{array}$ \\
\hline TikTak & $\begin{array}{l}\text { A project focused on collecting feedback data from users } \\
\text { and displaying it in a web dashboard [74]. }\end{array}$ \\
\hline Mezuro & A framework for monitoring source code metrics [65]. \\
\hline Monitoring system & $\begin{array}{l}\text { An online system where students can apply to be an as- } \\
\text { sistant teacher of regular courses [66]. }\end{array}$ \\
\hline System specialist in sport & $\begin{array}{l}\text { An application to enable the use of metabolic mathemati- } \\
\text { cal models for researches working with physiological data } \\
\text { [92]. }\end{array}$ \\
\hline Social networking startups & $\begin{array}{l}\text { A social network for Startup companies with the goal of } \\
\text { creating a community of highly connected and committed } \\
\text { entrepreneurs [74]. }\end{array}$ \\
\hline Family tree & $\begin{array}{l}\text { A genealogy community where each individual can create } \\
\text { a family tree - from time to time, the system attempts to } \\
\text { "link" the trees [74]. }\end{array}$ \\
\hline CoGroo & Portuguese grammar corrector used by LibreOffice [17]. \\
\hline
\end{tabular}

\subsubsection{The Informative Workspace}

Each team had its private informative workspace [71, [69] where they physically displayed technical debt items. In the XP Lab 2013 offering, all teams had a technical debt board (Figure 4.1). In the XP Lab 2014 offering, each team decided by themselves how to manage the technical debt items in their informative workspaces. Some teams decided to have the technical debt board and other teams kept the technical debt items list on a Kanban board.

Boards. In the technical debt board on the left, (Figure 4.1), a team placed the technical debt items that were incurred and/or identified. On the top of the board, there is a supply of blank 
cards called 'Fichas'. These cards were used to document the technical debt items. Figure 4.1 shows another team's boards, on the right, where they kept the list of technical debt items that were incurred and identified. On the right side it is possible to observe that they have a reserve of blank cards.
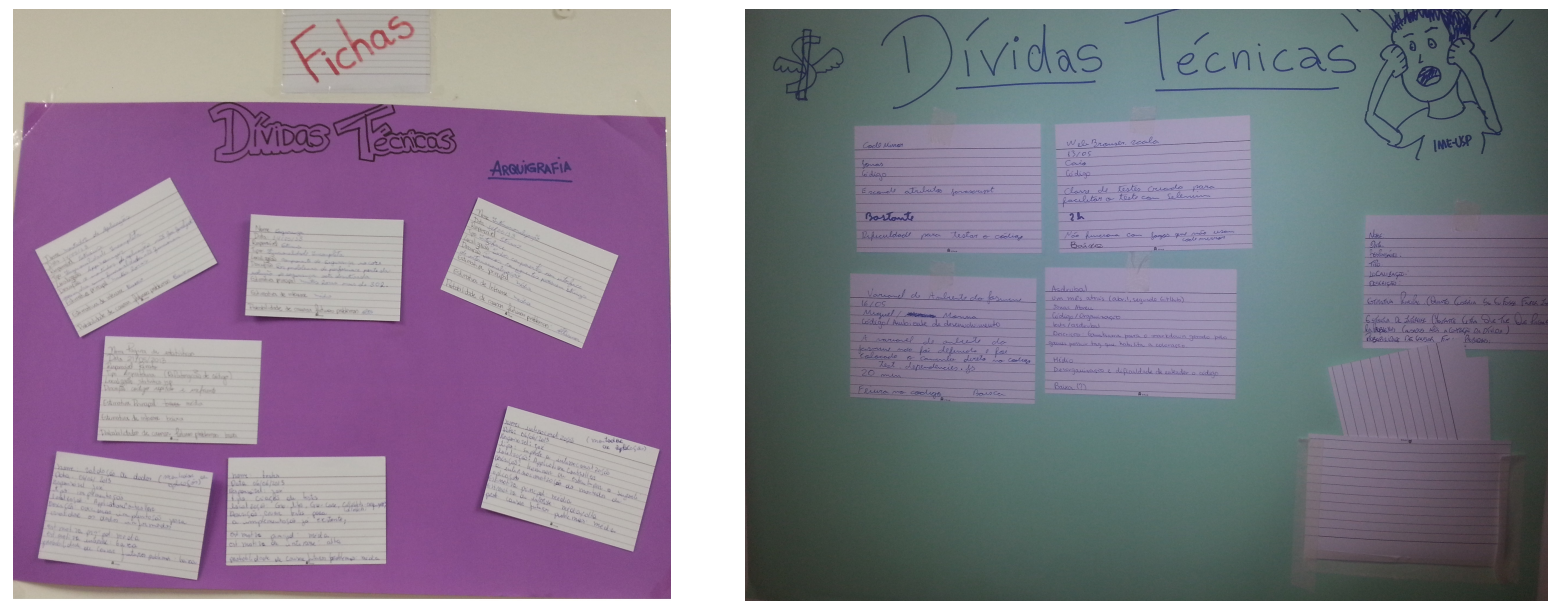

Figure 4.1: Technical Debt Boards

Cards. Each technical debt item was written on a card, which had to be filled out with the information about the technical debt item. The card structure was based on the template developed in the Guo and Seaman study [42. A model of the card can be seen in Figure 4.2.

Figure 4.2: Card with the Information about the Technical Debt Item.

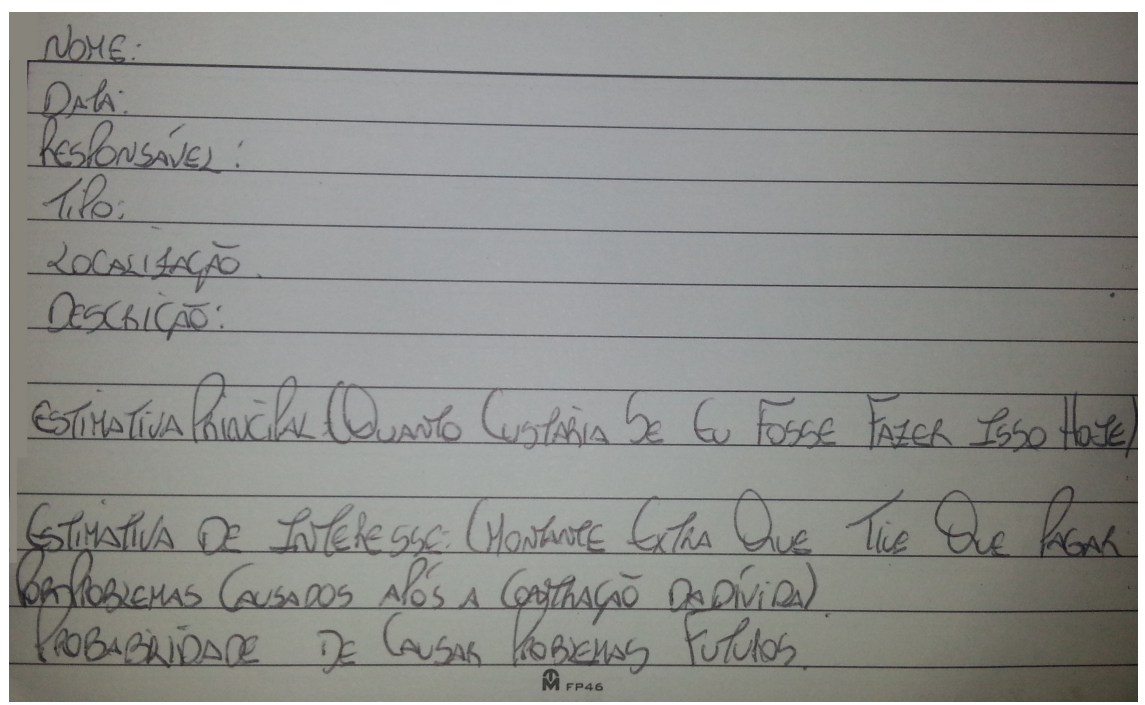

Source: 41]

As can be seen in Figure 4.2, a card should be complete for each technical debt item found, preferably filling all the fields below:

- Name: the name of the technical debt item;

- Date: date when the technical debt item was found or incurred;

- Responsible: person who found or incurred the technical debt item;

- Type: whether it was an architecture technical debt item, or documentation, bank, test, ...etc.;

- Location: place where the technical debt item is located - component, function, method, ...etc.; 
- Description: a brief description of the technical debt item;

- Estimated Principal: how long it would take to complete this task;

- Estimated Interest Amount: the extra work the team will have until the item is not be paid;

- Probability of Causing Future Problems: if the person believed that technical debt item would imply any problems, then he/she would classify the measure as low, medium or high.

If a doubt arose when the team member started to fill out the card, the person could either discuss it with the researcher or a team member, or even leave it blank.

In Figure 4.3 , we can see an example of technical debt item about duplicate code.

Figure 4.3: Technical Debt Item

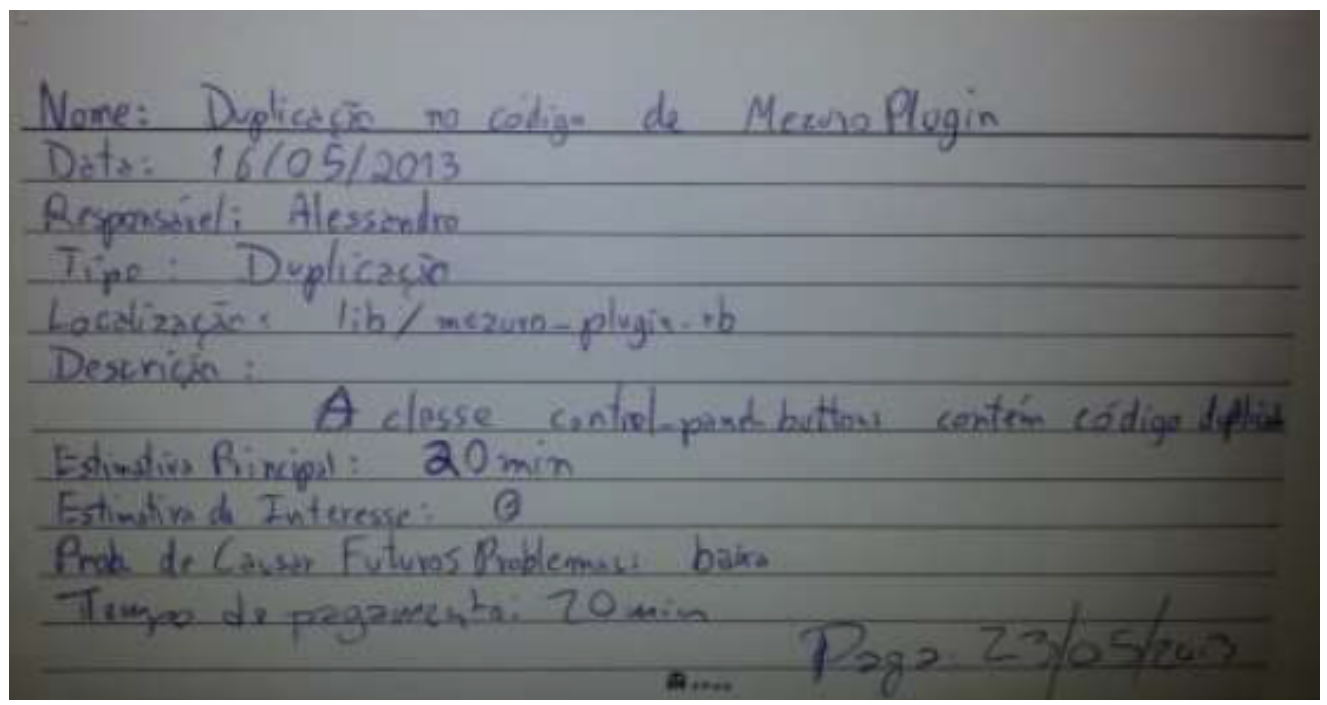

Source: Mezuro Team.

In this case the Name of technical debt item was Duplicate code in the Mezuro plugin, the Date (when the item was identified) was 05/16/2013, the Responsible (the person that incurred or found the technical debt item in this case was Alessandro, the Type was duplication (could be test, documentation, design, etc.), the Location (which part of the code the items was related to) was in lib/mezuro-puglin-rb. The Description (a brief description of the technical debt item) the class control-panel-buttons has duplicated code. Twenty minutes was marked as Estimated Principal, i.e. the amount of time the team expected to spend now if they implemented that task in the correct way - if they did not know how much time, then they could use a scale of high if they would probably spend a large amount of time on it, medium if not much time was necessary or low if they could probably solve it quickly. The Estimated Interest Amount should be filled out when they had pay the technical debt item. The Probability of it becoming a future problem (i.e. the interest probability) in this case was low. In this example, they also added in the card the Date when they paid the technical debt item, 05/23/2013, and how long it took them to pay off the item which was also twenty minutes.

These boards represent some of the boards used in the team's informative workspace. Some teams used a specific board to manage technical debt, as shown in Figure 4.1 while other teams used the Kanban board and put the technical debt items together with the tasks of the sprint. Notwithstanding, some teams also placed the list of technical debt items in the tool used to manage the project.

Tools. The teams had two code quality analysis tools available to use in their projects (they could also use other tools), one of which generated a specific technical debt index. As they were not required to use them, they did not necessarily consider them and their results. The code quality analysis tools were: 
- Code Climate: a tool for quality analysis of code repositories (https://codeclimate.com/).

- Sonar Qube: a code quality analysis platform that has a plugin that identifies technical debt items (http://www.sonarqube.org/ ).

\subsection{Research Design}

Data for this study were collected and analyzed through interviews and questionnaires. Before data collection, the teams spent some time identifying technical debt items in their projects. Below, we provide a description of how the technical debt items were identified and then we describe our data collection and analysis methods.

\subsubsection{Technical Debt Identification}

In the two offerings of the XP Lab, we followed slightly different steps to help the teams to identify technical debt.

\subsubsection{Extreme Programming Laboratory 2013 Offering}

In this study, we did the following steps shown in Figure 4.4 .

Figure 4.4: Steps of the XP Lab 2013 Offering
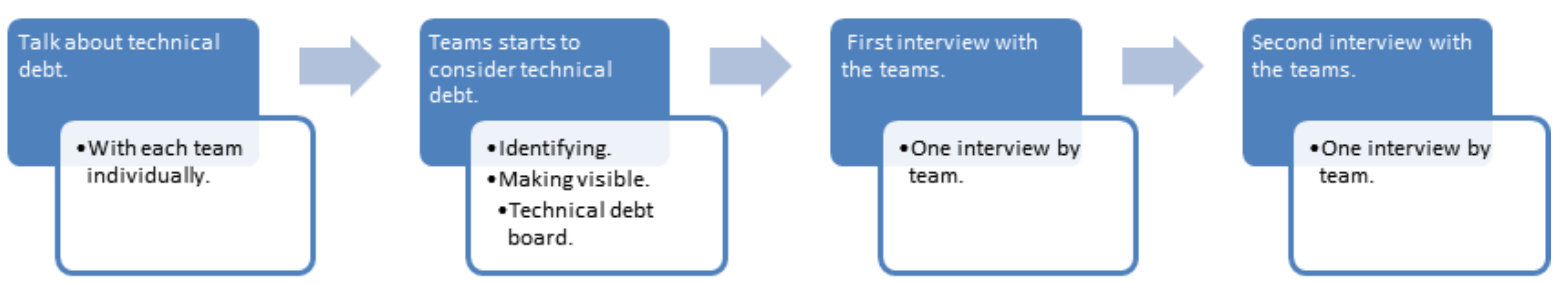

This course lasts about four months in the semester. In the first month, students begin to define their teams, choose their projects and make the first contributions to the source code. During this period, nothing was said about technical debt.

We gave a presentation on technical debt four weeks after the students had started working on their projects - as some students were familiar with the term - but others were not. The researcher presented the concept of technical debt in an informal conversation of approximately 40 minutes. After the presentation, we had a discussion and we encouraged the students to talk about their views on technical debt. We also talked about some concrete examples they had in their projects. The discussion lasted for about one hour and after that, each team had to prepare a technical debt board, where they had to document their technical debt items (Figure 4.3 shows an example of a technical debt item). Each technical debt item was written on a card on which they had to fill out a list of topics, after that it was then pinned to the board. The card structure was based on the template developed in the Guo and Seaman [42] study's. Each team had a board with a set of cards representing technical debt items. Every team that identified or incurred a debt put the information on the board. Given this first step, it was announced that the researcher would be available during 
the entire period of the course, as well as at other times to solve doubts and help the team with any difficulty.

\subsubsection{Extreme Programming Laboratory 2014 Offering}

This study was applied following the steps shown in Figure 4.5.

Figure 4.5: Steps of the XP Lab 2014 Offering
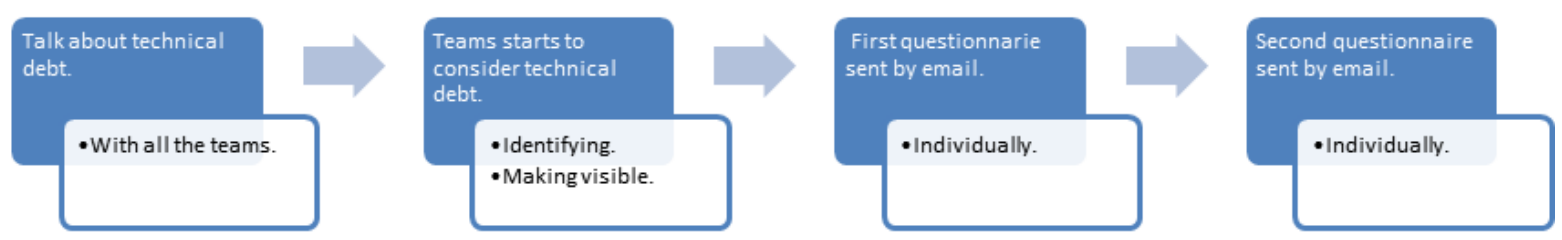

In the XP Lab 2014 offering, we made a presentation on technical debt for all the students fo the course (30 students) together for 30 minutes. It took place about two months after the course had begun. After the presentation, we discussed the subject for another 20 minutes, during this time the students could ask any questions they had about technical debt. Since the beginning of the course, the students had the code quality tools available, SonarQube and Code Climate (they could also use other tools). We did not impose the use of either boards or tools to identify technical debt. Instead, we showed them the meanings of technical debt items and some examples in each project. We also presented examples of a technical debt board and the categories they could use to identify technical debt items. Then each team decided whether they would monitor technical debt on their project.

\subsubsection{Interviews and questionnaires}

Data collection was done differently in the two XP Lab offerings. In both cases, similar data were collected both at the beginning and at the end of the course. We defined different approaches -interviews and questionnaires- to collected the data in both offering of the course due to data triangulation, aimed to reduce the bias. Once during interviews the team member's can not feel comfortable giving a real opinion on the subject. Given that, in the second offering of the XP Lab course, we collected the data by questionnaires.

\subsubsection{Extreme Programming Laboratory 2013 Offering}

Eight weeks after the teams started to identify technical debt items and fill the boards with cards, we carried out a face-to-face interview with pairs of students of each team. The pairs were commonly formed by members who were doing paired programming in that sprint and all pairs from each team were interviewed (As can be observed from Appendix A). For those team members that were working alone at that moment, the interview was conducted individually. The purpose of the interview was to verify the influence of technical debt visibility on the team. Also, it was our intention to validate whether everyone had the same understanding of the technical debt metaphor. In addition, we wanted to collect information about the team's vision on the process of identifying 
the technical debts according to criteria such as negotiation with customers, relevance, time spent, possible interference in the coding process and/or the development process. Asking questions was essential for validating the form of registration and identification in relation to factors such as unnecessary items, complexity and suggestions for improvements.

The interview was composed of twenty open-ended and multiple-choice questions separated into the following topic: 1 .

- The concept of technical debt;

- Were there any changes in the software development process?

- Negotiation with clients;

- About the experience of identifying technical debt;

- What is the relevance of identifying technical debt?

- What is the impact on software quality?

- Do the teams pay off technical debt?

- Will the teams pay off some technical debt?

Four weeks after the first interview, at the end of the course, we did a last open-format interview and we performed it for each team. In that last interview, each team was invited to talk about the experience of making technical debt explicit. Each interview took about twenty minutes. The aim was to collect the team's vision on the entire process of technical debt identification, as well as the impact on the team. During the interview, the researcher left the team members free to present their opinions and whenever she found it pertinent she made interventions aimed at collecting information about the criteria analyzed in the first interview. However, this time allowed each team member to freely include or improve any new criteria or item in their conclusions/observations.

\subsubsection{Extreme Programming Laboratory 2014 Offering}

In this offering of the course, we decided to apply a questionnaire to assess what each team member thought about technical debt (the questionnaire was answered individually by the students; this approach was taken as an attempt to decrease possible bias). The students were given the questionnaire one week before the class received a talk about technical debt. We sent a link to the questionnaire by email and then the students had one week to answer it. The questionnaire was composed of seventeen open-ended and multiple-choice questions, separated into the following topics:

- Software quality;

- What does the team do about quality?

- Familiarity with technical debt;

- Do you know about and use the technical debt concept?

- How is technical debt used in the project?

- Are you using the Sonar Qube or the Code Climate report?

- Are you using a technical debt list?

\footnotetext{
${ }^{1}$ It is possible to access all the questions in the following link https://goo.gl/BMmrKH

${ }^{2}$ It is possible to access all the questions in the following link https://goo.gl/i3EL16
} 
- Have you paid any technical debt item?

- Is there any evidence that having the technical debt items visible has any influence on the team?

- Did you identify any technical debt item that was not identified by the tools?

- Are you going to consider technical debt in future projects?

- Do you think the technical debt concept is relevant?

The same questionnaire was applied a second time at the end of the course. The aim was to see if there was any change in the team members' behavior.

\subsubsection{Data Analysis}

For the data analysis, we used coding techniques from the Grounded Theory approach [19], [97. Grounded Theory methods are aimed at building or discovering a theory. In this approach, the data analysis proceeds in three interdependent steps: open coding, axial coding, and selective coding. In the open coding step, the researcher interprets the data to identify patterns and define codes, "... event/action/interaction, are compared against others for similarities and differences; they are also conceptually labeled [...] conceptually similar ones are grouped together to form categories and their subcategories..." [19. In axial coding, "categories are related to their subcategories, and these relationships tested against the data..." [19]. Then in selective coding, "all categories are unified around a central 'core' category and categories that need further explanation are filled-in with descriptive details..." [19].

For data analysis, we used the $\mathrm{NVivd}^{3}$ tool, which is widely used for analysis of qualitative data. In this case, the goal was not to use Grounded Theory to develop a new theory but only use its coding steps to answer our research questions.

We also analyzed the source code of the projects with the SonarQube and Code Climate tools to try to identify relationships between team's beliefs and the reports from these tools.

\subsection{Results}

In this section, we describe our findings first separated by each offering of the course, 2013 and 2014, to discuss specific items of each interview and questionnaire. Then the results of the two offerings are organized by the coding steps. As the main question in both offerings of the XP Lab was the same and the results obtained were similar, we analyzed the results of both offerings together.

\subsubsection{Extreme Programming Laboratory 2013 Offering}

At the beginning of the first interview, team members were asked about their understanding of the concept of technical debt, Figure 4.6 .

To the team members, the concept of technical debt was directly related to issues such as: a system failure that needed to be corrected, a 'gambiarra that had to be refactored in the future, a problem in the code that had to be improved as in; "[...] it is a problem that you decide to leave aside and solve later and that can bring us some damage, is not it?! With the deadline, but it does not appear to be the best moment to deal with it...", or a pending in the code that needed to be solved.

\footnotetext{
${ }^{3}$ http://www.qsrinternational.com/support/downloads/nvivo-9

${ }^{4} \mathrm{~A}$ brazilian expression. It basically means to use improvised methods/ solutions to solve a problem.
} 
Figure 4.6: Technical Debt Concept

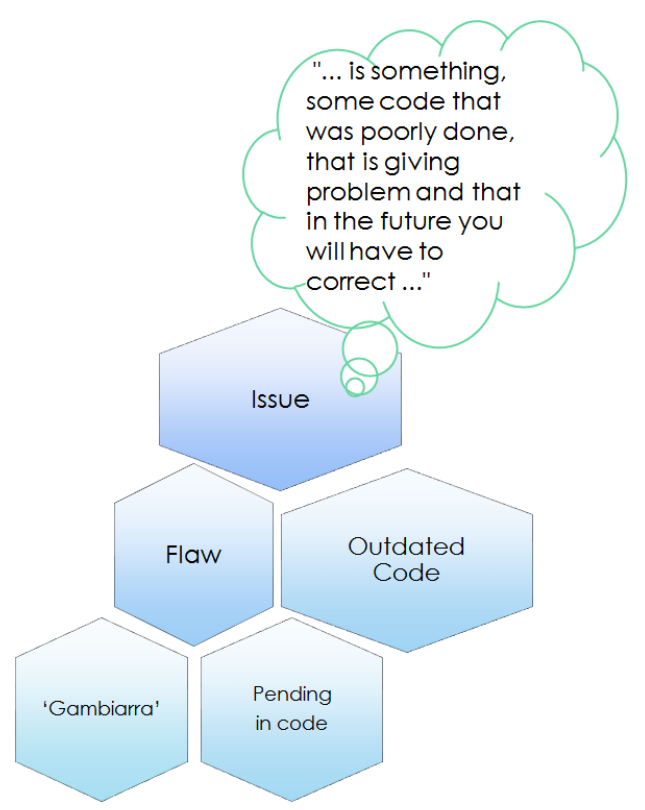

When we asked them if before the talk about technical debt the team already considered the concept of technical debt, most of them indicated that it was not considered, as can be observed from Figure 4.7

Figure 4.7: Percentage of People Who Considered Technical Debt in their Projects.

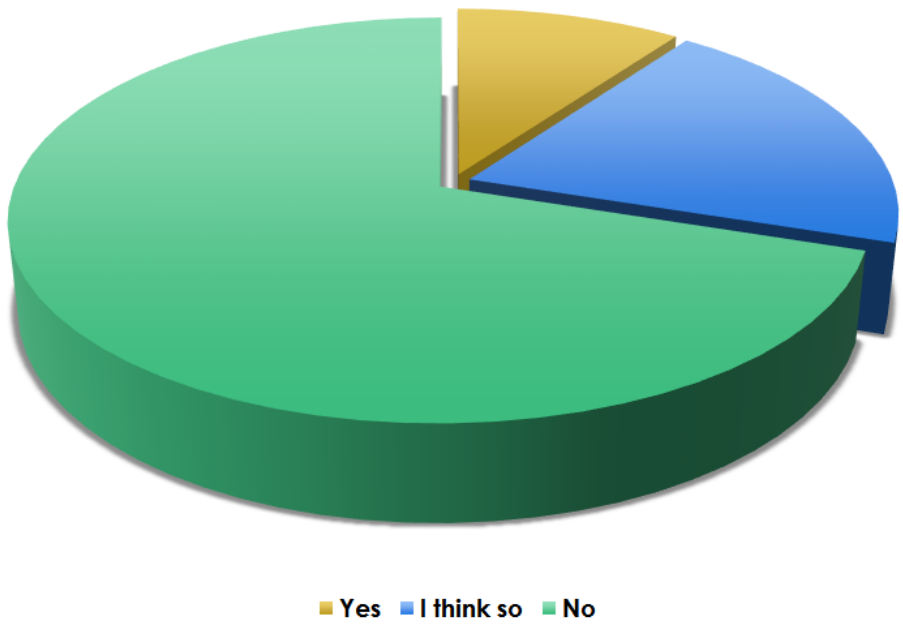

It may also be noted that a small part of the members considered the concept of technical debt, for instance: "[...] it is something that you always have in mind...". It is that even when you are writing a test and there are some parts of the code that you know that it is [...], it is very complicated to test that part [...] I did not call it technical debt, I did not have all this thinking, but I already had this idea of 'Ahh, if I make it visible here it will bother me and at some point I can solve it' [...] ". A small portion believed that they actually considered the technical debt concept, for instance, "I think so, I think this is natural in fact ... " and "Yes, we try[...] what we cannot do, we do not remember all of it because it is not documented $[\ldots]$ ". And everyone said that in most cases they either forgot about it or it did not matter, "Normally when you incur a debt, you say '[...] I need to go back' and forget about it, you go on ...".

When the team members were questioned about the card structure - properties, fields, infor- 
mation - to register the technical debt item Figure 4.2, they highlighted it as a positive point the fact that they did not need to spend much time to identify and register them. However, some of them mentioned that it was only necessary to insert in the card information such as: identification, type and location. In addition, they thought it was complex and a bit confusing to define the estimates, "I think estimates are always difficult. We made the plan [...] we still have disagreements about estimates, considerable disagreement [..] ". As a matter of fact, some developers pointed out that they considered very interesting the idea of using a technical debt board such as in Figure 4.1. Thus, they felt uncomfortable when they saw that they were accumulating technical debt items. They were encouraged to focus on paying some of the technical debt items. It was too disturbing for them to work on the project and see that it was fraught with problems. If the health of another team's project was visibly better, it was even more upsetting.

However, it should be emphasized that over time they may become accustomed to the board and ignore it. They also related that in the past, they consciously incurred technical debt items many times, but usually those items were forgotten. After taking technical debt into account, they would come across the technical debt items at any moment and they could also track them. It is encouraging for them to perform the refactoring at any given moment, "Track and [...] do lots of refactoring, you will feel somehow forced to solve it...".

\subsubsection{Extreme Programming Laboratory 2014 Offering}

In the first questionnaire we obtained 14 responses and in the second one, we had 11 respondents. In the analysis of the data, it was identified that the great majority of respondents affirmed that they were familiar with the technical debt concept. However, only two teams in fact, considered taking into account and listing the items both in the tool they used to manage the issues and on the task monitoring board. It was also found, that the other team members who said that they knew the technical debt concept did not actually consider it, but understood that they had some points in the project that should be improved and that they acknowledged these as technical debt items. One respondent said, "[...] in our case, we have too many working hours (overtime) to be done, which may constitute an important part of our technical debt.". Other answers indicated that many of them still might not have quite understood the concept: "No. Our project has not got any technical debt items yet." and "No. Very difficult, abstract to manage...", whereas, for example, this project had no test. It is also possible that they had not spent too much time to identify the technical debt items in order to prioritize other activities, "No, because the group is focused on customer delivery. ".

When analyzing the answers to this second questionnaire, it can be verified that the teams have come to consider the available tools. One team added another code analysis tool called EclEmmahttp://eclemma.org. Another team used the JUnit https://junit.org/junit5/framework. and other team used the framework RSpec http://eclemma.org.

However, the vast majority only used this tool to verify test coverage. Some of them mentioned that they also considered the tool to monitor complexity and duplication. It was observed that new items that focused on the quality of the project started to be considered. The teams began refactoring, testing, automated testing, programming in a standardized way, and running the code review. Furthermore, some team members mentioned that they were consulting and talking more to the customer. It should be noted that this improvement probably happened due to the influence of the good practices suggested by the Extreme Programming methodology, which are encouraged throughout the project. In addition, throughout the project, they have some items by which they are evaluated, doing automated tests was one of them. As they are encouraged to do at least one refactoring day, that may justify the quote about refactoring.

The technical debt items pointed out by the tools were considered relevant, mainly because they can hinder the understanding and the evolution of the project, as can be observed from 
the quote: "It is certainly relevant, because, in a way, it indicates how far the code is from the ideal as it allows the code to be extended in the future without major difficulties (avoid the snowball effect and the difficult interpretation of the code).". Since they started to identify the technical debt items it was possible to define actions to improve the quality of the software, "The identification of technical debt items allows us to have a better idea of what should be done to improve the quality of our software. "

Another factor worth mentioning is that all the respondents indicated that the project had many other technical debt items that were not found by the tools. Regarding the tools, they pointed out, "... but they are not enough. They point to logical flaws in our code, but they do not cover every case.". The team members reported some examples of technical debt cases which were not identified by tools, for instance patterns in the nomenclature of variables and functions, "[...] code formatting and clarity of nomenclature of variables and functions.", difficulty in adapting a technology, to achieve a certain goal, "[...] it was impossible to use selenium to create acceptance tests for them ..."; pending between database models, problems defining data types such as "... leave the variable department as string or as an entity in the data bases ..."; security flaws and user interface problems, "[...] non-practical UIs".

According to the interviewees, the fact of having shown the technical debt items encouraged the team to consider them and made the team embrace some attitudes such as:

- Refactoring: the team started to do more refactoring in the project code. They also had more moments to discuss whether they would pay them "[...] increase frequency of refactoring";

- Improvement in the code documentation: the documentation started to address the relation of some of the problems of the code such as location, brief description about, etc.;

- Discussion before incurring technical debt: before deciding whether to incur a debt the team discussed the subject with the group, "Try to avoid creating them by asking for group opinions";

- They made an effort to develop better in the first time;

- Developing with more care/attention: while developing the code, they were worried about whether they were incurring a technical debt item;

- Discussing/thinking more about the real meaning of the requirement;

- Establishing some criteria to avoid incurring many technical debt items;

- Improving priority setting;

- Encouraging the team to resolve failures;

- Using refactoring day to pay off some technical debt items;

- Debating about the technical debt items in the iteration planning, "We remember them and take them into account in planning the iterations";

- Evidencing technical debt items when those were identified, by inserting them into the technical debt board, "We put everything we find out of debt on the board so that it would be paid";

- Increasing the concern about the quality of the code: they began to do more tests, "Try to increase the number of tests where it was necessary." and "Worry about quality once others in the group can see the quality of your code".

When the respondents were asked if they would work with this concept in other projects, all respondents said yes. Their reasons were:

- Improvement in communication as can be observed from the following report: "Yes. Although intuitively avoiding technical debt items before knowing the term, a better understanding and definition have brought benefits to the team's communication"; 
- Possible to see the impact on the future of the project: "... because we feel like this can be a problem for the future of the project ...";

- Knowledge of what needs to be improved in the software: "Identifying technical debt items allows us to have a better idea of what should be done to improve the quality of our software ...";

- Code and project quality control: "... as for the quality of the developed system ...", "I will consider therefore, that I can control the quality of the software ...";

- Learning over time: "... because this way, it is possible to improve our quality as programmers and how we evaluate the code of others developers...";

- Communication, were concerned to make the new features clear to the other team members";

- The code quality is seen more closely.

In the next sections, we describe the results of Grounded Theory analysis, organized by coding steps. We analyzed the results of both offerings together.

\subsubsection{Open Coding}

In this step, the data analysis was conducted by reading the transcripts of the interviews and the answers to the questionnaires. We applied the coding process to this material, line by line. In this phase, we discovered the open codes. In Table 4.2, we list three code samples 5 .

Table 4.2: Extreme programming projects.

\begin{tabular}{|l|l|}
\hline Open Codes & What They Talked About \\
\hline $\begin{array}{l}\text { Changed attitude of the } \\
\text { teams }\end{array}$ & $\begin{array}{l}\text { The teams discussed more the tasks they have to do before } \\
\text { incurring a technical debt item and they thought more } \\
\text { before taking the decisions. }[3]\end{array}$ \\
\hline Communication & $\begin{array}{l}\text { After the identification of technical debt items, the teams } \\
\text { had more discussions. }\end{array}$ \\
\hline Maintainability & $\begin{array}{l}\text { The identification of technical debt items helps the teams } \\
\text { to know that there will be some changes in the software } \\
\text { in the future. }\end{array}$ \\
\hline
\end{tabular}

\subsubsection{Axial Coding}

During axial coding, the open codes were reassembled in new ways to form categories. The goal was to create a higher abstraction level of meaning. Thus, codes were grouped to form subcategories, and in turn, they were organized into categories. This process was highly iterative, with codes and categories forming and re-forming as more data were incorporated into the evolving understanding [19].

In Figure 4.8, it is possible to observe the list of categories and subcategories resulting from axial coding analysis. The first level is the resulting main category, which merged from the subcategories of the second level. The subcategories resulted from the codes emerged in the third and fourth levels.

When we make a list of technical debt items, one of the most important influences is the attitude of the team (team behavior), "... registers by not forgetting, there was a change in the attitude of the team..." and "... increased people's concern regarding the technical debt...". The team had less 'untouchable' expert professionals, and behaved more as a team, rather

\footnotetext{
${ }^{5}$ It is possible to access all the codes in the following link https://goo.gl/vyTCwg
} 
than a group of individuals. They talked more about the technical debt items "... we discussed about these debts. Otherwise, the project would not have advanced..." and really thought about the necessity of incurring it. It helped them to have the same understanding of the concept of technical debt because they discussed it (technical debt concept). In addition, if the team members were not sure whether to incur a technical debt item, the team member would debate with another team member to help him/her make this decision, thus improving the team's communication. Team members started talking more to each other and because of it, they knew what part of the project was being modified and the problems of the software, "It was easier to remember that we have to fix things, debts...". Furthermore, if the team's communication was good, they were more comfortable to share their difficulties with each other. After the team started, to identify technical debt, developers discussed their decisions rather than just did something and moved on. Now that all the software problems were clearer to the team members, "usually only the person thought or knew about it (...), now with the technical debt, it becomes clearer as well...". They began to argue among themselves, before incurring a debt, "As evidenced here, we even got everyone talking about the debts instead of just attempting a quick fix and moving on."

They started to think more about the necessity to incur some technical debt items. Several times they concluded that it was not required. A team member says: "From the moment I started to think about that item, which was debt, I asked myself what was the current cost compared to the future cost. Because if the cost of doing it now is lower, then it's better to fix it now...". Another student says: "... we think twice before making a technical debt.".

When technical debt items were visible, the team had more control over whether they would pay off the technical debt item, whether they would incur more technical debt items, or whether they would incur and pay later. Additionally, it facilitated planning to repay the technical debt items, "We have analyzed some of the debts, now we will plan what we might kill, kill some of those debts, then it becomes easier to make this analysis...". Therefore, if they incurred a technical debt item, they would make it visible, monitor it, and sometimes they would look back at this technical debt item. This way, they always thought about continuous improvement. Thus, the team could define a strategy to pay down some of the technical debt items to improve the code quality.

Figure 4.8: Categories and Subcategories of the XP Lab 2013 and 2014 Offering

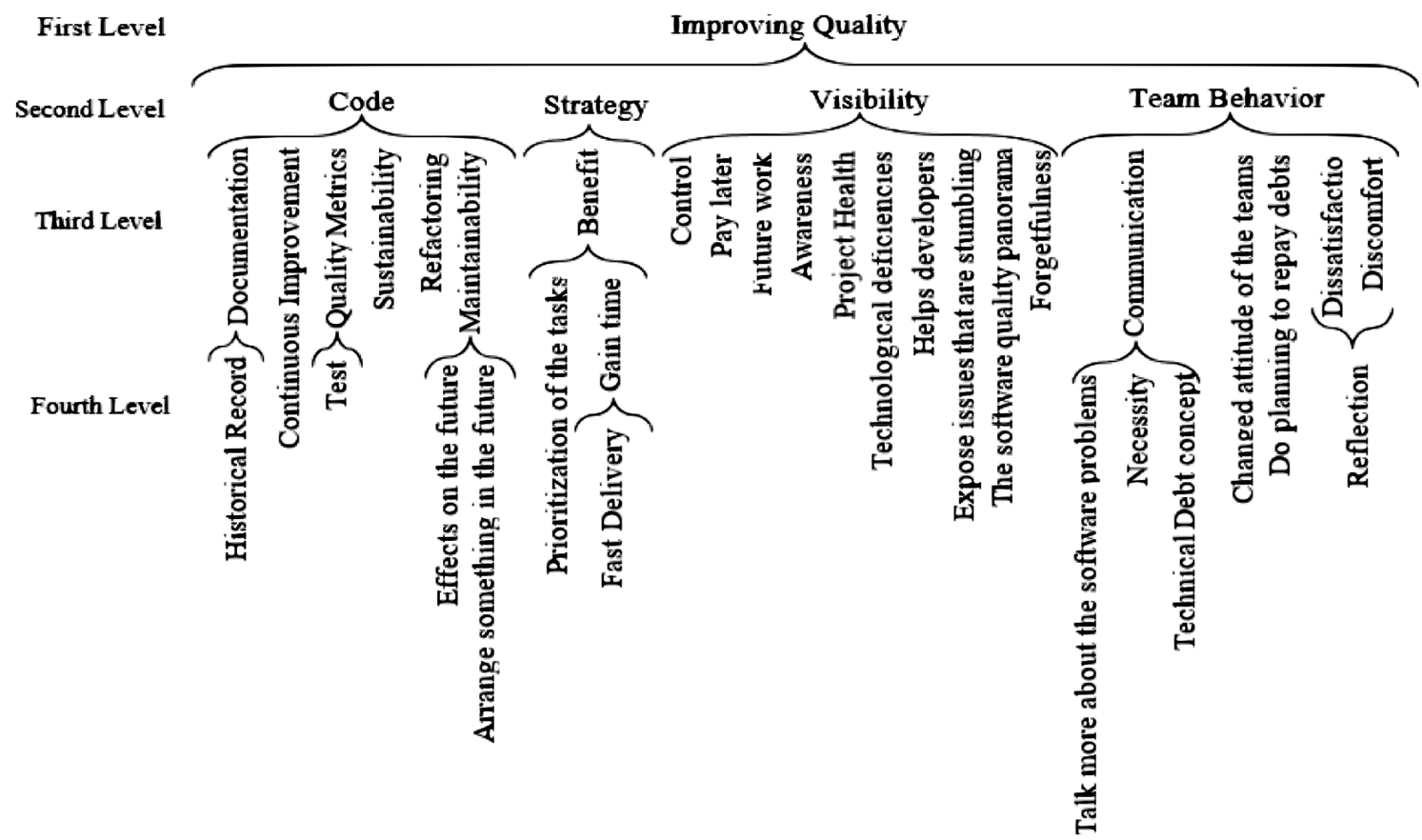


On the other hand, if a team member incurred a technical debt item and never paid it, the project would probably lose quality. Nevertheless, they might incur technical debt to prioritize other tasks or as a business decision to deliver fast and then that decision would have been made strategically.

The technical debt item list presented indications on whether the code quality was improving and helped them to understand the current development state. It also indicated if they would have a lot of future work and future refactoring to do in that code. Indeed, if the software had technical debt items, the team would probably need to perform some refactoring and it directly affected the sustainability of the project. Furthermore, the team could do an analysis of the technical debt items and they could be define a software's quality metrics to help them monitor the software quality, for instance, test. They could also identify technological deficiencies and define possible directions to improve their technical skills so as to not repeat the same mistakes, in order to mitigate the occurrence of those recurrent technical debt items that were recurrent.

The teams used the technical debt item list as documentation providing a historical record of the immature parts of the project. That said, each team knew that some parts of the project should be improved, as it was possible to see if there was a technical debt item to pay off. This documentation helped the teams to maintain the code and it reflected on the health of the project. Likewise, it affected the teams by sometimes causing dissatisfaction with the quality of the software. Many team members saw that it was very uncomfortable to arrive at work and see that the health of the project was not so good. If the source codes health of the other teams were good, it was even more uncomfortable. Therefore, this process of the team having discussions and presenting dissatisfaction impacted on the following reflection: when a team member decided to incur a technical debt item, thought about it and discussed it, it demonstrated that he/she had a good understanding of the problem that he/she had to solve. This often resulted in the non-insertion of a technical debt item in the code, since the developer only lacked the understanding of what should be done.

Considering technical debt implied generating a culture focused on quality and it affected factors related to project continuity. It has an influence on the cost and the viability of maintainability and evolution of the project. A developer said that, if they did not have the technical debt items visible it was too difficult to identify the software quality landscape, "It was difficult for you to identify the whole landscape...". That way, if in the future the team needed to make some changes in the legacy code, they already knew what were the issues. It provided a general awareness to the team about the problems of the software. It also might help a new team member that does not know the code to have a notion of the code quality and the location of the code problems.

\subsubsection{Selective Coding}

Selective coding constituted the third stage of data analysis, with the objective to refine and integrate categories and unveil a category deemed as central, encompassing all the others. The full potential of abstraction was employed to incorporate the full scope of the investigated and coded data [19], [96].

In our case, the objective was not to generate a theory, but rather to identify the main categories. The aim was to describe the impact of technical debt awareness.

The categories Strategy, Team Behavior, Code, and Visibility represent the main influences on teams due to making technical debt items explicit. Each of these categories captures part of our results, although none of them describes the phenomenon entirely. For this reason, another abstract category is required, a conceptual idea on which all categories are included. As such, we concluded that the resulting core category might be a perceived notion on "Improving Quality". 
All teams progressed towards creating a culture of quality of the code, team, and project. This arose primarily because each team member started to think more about the need to incur technical debt items. Many times they decided that incurring technical debt was not necessary in a given situation. When a team member was not sure about the necessity or not to incur the debt, they spoke with other members to make a decision. This way, the team improved their communication and it became clearer what each member was doing. Consequently, it was easier to understand the objective of the project. Making technical debt explicit had a direct implication on the team behavior. Most of the team members said that after they started to identify technical debt, communicated more effectively, thought more about the real need to incur debt or not, discussed more about code quality, refactored some parts of the code more frequently, as well as they were aware of the code problems were and where each team member was working at any time on the project. Equally important, when communication among the team was good, people felt more comfortable to expose and discuss their problems with the team. The team then became more an actual group working together, rather than a group of working separately on different parts of the system.

In some situations, incurring some technical debt items was a Strategy to gain time, due to the time to market. In effect, if there was a list of technical debt items items, in the future it might be possible to correct them by refactoring. The list of technical debt items was used as documentation, which enabled the historical record of the technical debt items list that the code contained. When the need to change a particular part of the system appeared, it was possible to verify if it had some technical debt item and if this debt would affect such functionality. In that case, the team members had the option of paying it off or not. The documentation helped in the visibility of the project's health. If the software had any technical debt, it probably would have more deficiencies.

Finally, once a team had the technical debt items list, they automatically became Aware of the technical debt items of the project, that impacted on the software quality. As a result, the team could think carefully before possibly incurring another technical debt item. The team could decide when and where they would improve the software quality.

The teams had similar views on the importance and benefits of making technical debt explicit. A significant finding is that the teams considered it very helpful because they could see the whole landscape of the software quality (they knew which part of the software had immature code). They also emphasized that it was very useful to have a board that showed daily information on the codes health. Before becoming aware of technical debt, the team members reported that they sometimes incurred technical debt but never remembered to go back and correct it. In contrast, after considering technical debt, they thought about the necessity of incurring technical debt and often decided against it. Also, they could see the technical debt list and so they did not forget about the technical debt items that needed to be addressed. They discussed more about how to implement the tasks and they talked more about the problems of the software because their list of technical debt items was visible. This process of thinking about incurring or not TD, discussing it and reviewing the TD during the project can create a culture focused on improving the software quality.

In addition, in this study we explored some ways of identifying and monitoring technical debt. Our subjects found that keeping a technical debt board was very useful for documenting technical debt, making it visible, and adjusting the technical debt board and their behavior accordingly. By using the technical debt board, they are always aware of the list of deficiencies so they have a constant reminder of how to organize their work in order to improve the software. As a complementary aid, they may use tools to help them to identify and monitor the technical debt occurrence. However, it is important to highlight that tool reports provide a static analysis of the software quality and some technical debt items cannot be identified using static metrics. The results of this study could motivate teams to consider technical debt further and to help developers convince leaders and directors, the decision makers, to start 
considering technical debt. These approaches used by the XP Lab teams such as boards, cards and tools can help teams in companies to deal with technical debt. In addition, they could define the list of technical debt items that are crucial to the project but hard to identify with the tools. Ultimately, over time they can define a strategy to deal with the technical debt over time.

\subsubsection{Tool-Based Static Analysis Results}

In the two editions of XP Lab, we used the Code Climate and Sonar Qube tools to analyze the code of the projects. We considered the Code Climate metric, grade point average (GPA) and in the Sonar Qube, the following metrics: lines of code, code smells, duplication rate, technical debt rate, duplicated blocks, bugs and vulnerability.

In the XP Lab 2013 offering, we analyzed one project using the Code Climate tool (that supported the language used in the project, in this case, Ruby). Further, we analyzed three projects with the Sonar Qube tool.

In XP Lab 2013 edition, as we can see in Figure 4.9, during the Sonar Qube analysis of the following projects: Game VidaGeek, Tiktak and Arquigrafia we did not identify large variations in the measured metrics when comparing the two different versions of each project (before and after the teams considered technical debt). In the Game VidaGeek and Tiktak projects the number of code smells, bugs, vulnerability and lines of code increased. However, in both projects the percents of duplication decreased. In the Arquigrafia project, the technical debt rate and the number of bugs decrease. In fact, the changes were not significant in all projects and we can not assume that these changes occurred because the teams considered technical debt.

Figure 4.9: XP Lab 2013 Edition

\begin{tabular}{|c|c|c|c|c|c|}
\hline XP Lab Edition & Project & Tool & Metric & First Version & Last Version \\
\hline \multirow{22}{*}{2013} & Mezuro & Code Climate & Grate Point Average & 1.73 & 1.73 \\
\hline & Game VidaGeek & Sonar Qube & Lines of Code & 3.800 & 4.000 \\
\hline & Game VidaGeek & Sonar Qube & Code Smells & 844 & 850 \\
\hline & Game VidaGeek & Sonar Qube & Duplications & 1 & 0 \\
\hline & Game VidaGeek & Sonar Qube & Debt & 12 & 12 \\
\hline & Game VidaGeek & Sonar Qube & Duplicated Blocks & 2 & 0 \\
\hline & Game VidaGeek & Sonar Qube & Bugs & 397 & 403 \\
\hline & Game VidaGeek & Sonar Qube & Vulnerability & 2 & 8 \\
\hline & TikTak & Sonar Qube & Lines of Code & 1.700 & 1.900 \\
\hline & TikTak & Sonar Qube & Code Smells & 145 & 178 \\
\hline & TikTak & Sonar Qube & Duplications & 40.3 & 35.3 \\
\hline & TikTak & Sonar Qube & Debt & 3 & 3 \\
\hline & TikTak & Sonar Qube & Duplicated Blocks & 20 & 20 \\
\hline & TikTak & Sonar Qube & Bugs & 16 & 22 \\
\hline & TikTak & Sonar Qube & Vulnerability & 20 & 27 \\
\hline & Arquigrafia & Sonar Qube & Lines of Code & 24.000 & 28.000 \\
\hline & Arquigrafia & Sonar Qube & Code Smells & 1.900 & 1.900 \\
\hline & Arquigrafia & Sonar Qube & Duplications & 50.9 & 51 \\
\hline & Arquigrafia & Sonar Qube & Debt & 41 & 40 \\
\hline & Arquigrafia & Sonar Qube & Duplicated Blocks & 21 & 21 \\
\hline & Arquigrafia & Sonar Qube & Bugs & 662 & 661 \\
\hline & Arquigrafia & Sonar Qube & Vulnerability & 50.9 & 51 \\
\hline
\end{tabular}

In the Mezuro project, the GPA before and after the team started to consider technical debt there was no change.

\footnotetext{
${ }^{6}$ This is the metric resultant from the Code Climate tool analysis (https://docs.codeclimate.com/docs/ code-climate-glossary\#gpa)
} 
The fact that some metrics have not changed can also be due to the short period of time during which the team developed the code after they had started considering technical debt.

In the XP Lab 2014 offering, we analyzed two projects with the Code Climate tool and three projects with the Sonar Qube, as we can observe in Figure 4.10.

Figure 4.10: XP Lab 2014 Edition

\begin{tabular}{|c|c|c|c|c|c|}
\hline XP Lab Edition & Project & Tool & Metric & First Version & Last Version \\
\hline \multirow{23}{*}{2014} & Mezuro & Code Climate & Grate Point Average & 3.78 & 3.75 \\
\hline & Monitoria & Code Climate & Grate Point Average & 3.42 & 3.42 \\
\hline & Specialist in Sport & Sonar Qube & Lines of Code & 13.000 & 32.000 \\
\hline & Specialist in Sport & Sonar Qube & Code Smells & 1.200 & 2.000 \\
\hline & Specialist in Sport & Sonar Qube & Duplications & 0.8 & 19 \\
\hline & Specialist in Sport & Sonar Qube & Debt & 23 & 43 \\
\hline & Specialist in Sport & Sonar Qube & Duplicated Blocks & 8 & 225 \\
\hline & Specialist in Sport & Sonar Qube & Bugs & 412 & 838 \\
\hline & Specialist in Sport & Sonar Qube & Vulnerability & 8 & 12 \\
\hline & FamilyTree & Sonar Qube & Lines of Code & 2.500 & 3.000 \\
\hline & FamilyTree & Sonar Qube & Code Smells & 303 & 527 \\
\hline & FamilyTree & Sonar Qube & Duplications & 0 & 2.3 \\
\hline & FamilyTree & Sonar Qube & Debt & 5 & 8 \\
\hline & FamilyTree & Sonar Qube & Duplicated Blocks & 0 & 8 \\
\hline & FamilyTree & Sonar Qube & Bugs & 12 & 18 \\
\hline & FamilyTree & Sonar Qube & Vulnerability & 3 & 4 \\
\hline & StartUp Social Network & Sonar Qube & Lines of Code & 140.000 & 142.000 \\
\hline & StartUp Social Network & Sonar Qube & Code Smells & 10.000 & 10.000 \\
\hline & StartUp Social Network & Sonar Qube & Duplications & 12.9 & 8.6 \\
\hline & StartUp Social Network & Sonar Qube & Debt & 217 & 224 \\
\hline & StartUp Social Network & Sonar Qube & Duplicated Blocks & 2.700 & 2.500 \\
\hline & StartUp Social Network & Sonar Qube & Bugs & 3.400 & 3.600 \\
\hline & StartUp Social Network & Sonar Qube & Vulnerability & 50 & 56 \\
\hline
\end{tabular}

The result of the analysis of the Mezuro's code was 3.78 GPA and after considering technical debt the GPA decreased to 3.75, This project is a specific case because the students started to consider technical debt in the XP Lab 2013 offering and still considered technical debt during the 2014 offering. Considering it, whether we compare the first analysis of the Mezuro code to the second analyze (1.75 to 3.75 ) we can observe a significant increase of the code quality. But, it is important to note that most of the team members did the course for the second time, which may influenced the improvement in the code quality.

In the Monitoria project we cannot identify any change.

In the Specialist in Sport all the metrics increased significantly, indicating a lower level of quality. In this project it may have occurred because the customer had required many new features to the team before the course ended. We can observe that the lines of code increased from 13.000 to 32.000 .

The analysis of the project FamilyTree reported that all the indexes increased, which can also represent a deterioration in the quality level. The analysis of the Startup Social Networking showed that the indexes, number of bugs, the vulnerabilities and the technical debt rate increased. In addition, two indexes decreased, the rate of duplication and the duplicated blocks.

It is important to highlight that the students reported that most of technical debt items were not identified by the tools, which could explain the modest size of the changes in these metrics. For instance, one team was using Handsontable 7 for data entry and they had a problem with the validation of the data. Neither the presence of this type of technical debt item or the impact of paying it off, could be identified with Sonar Qube or Code Climate reports. In general, many types of technical debt items (e.g. those related to business rules) cannot be identified through static metrics. 
Other characteristic of this study that may have influenced the small changes in the analysis of the reports, is that most of these projects were developed only for a few months, and did not have major changes in the code. In the long-term, it would probably be possible to see more significant differences comparing the software quality by the analysis of the tool reports. Also, new tools to manage technical debt have been developed, it could be interesting to compare the analysis of these tools with others approaches to manage software quality. Comparing metrics that can identify other types of technical debt items that can not be identified by static metrics. Another study that can be conducting is to analyze the influence of consider technical in others aspects of the team that indirectly could affect the software quality, such as the team communication during the design of the software architecture or the increase of the productivity of the team due to the low complexity of the code.

\subsubsection{Threats to the Validity}

In this study, we took some actions to mitigate possible biases. We explain them in the following points:

- Construct validity (credibility): We used multiple data collection approaches in order to reduce possible bias. When planning the interviews and the questionnaire we discussed the best way to formulate the questions. As a pilot test we did a first interview and questionnaire with one member of the group as a pilot test. Based on this test, we reviewed the questions. We did not include these data in the final analysis. One important issue to highlight is that, during the interviews and questionnaires, the students might not have understood correctly the central meaning of the questions. Because of this, in the interviews, if the student did not understand the question, the interviewer explained it to them. The researcher was available throughout both studies if the students had any questions.

- External Validity: This study can be replicated in other academic courses, as well as in companies. In both cases, the study can be separated into two parts and can be applied in these situations: one within teams that do not consider technical debt yet, to verify whether the awareness of technical debt influences any aspect in the team such as communication. Furthermore, this study can be analyzed with teams that already consider technical debt and its identification, monitoring and management in order to investigate whether it is possible to identify some changes in the team behavior and in the software quality. If they have a historical record, we could also use tools to measure the software quality. Finally, to carry out this study it is not necessary to makes it significant changes in the team's environment, which makes feasible to replicate the study in companies.

- Internal Validity: We analyzed the data separately, first the interview transcriptions, then the questionnaire responses, and lastly we compared and merged the findings that were relevant and presented plenty of evidence in the results of both studies. In case of doubt about a specific point, we went back to the data and re-analyzed them. After that, the advisor and co-advisor read the results and if they indicated some points to be re-analyzed, the researcher would re-analyze the data. We did analysis and re-analysis many times until we were sure of the conclusions.

- Reliability: To interpret the data we followed the coding techniques from the Grounded Theory steps. Although the data analysis was made by a single researcher, however the results of the analysis were discussed by the two researchers and with the advisor and co-advisor and every time a doubt emerged, we re-analyzed the data. Also, this study is a result of two years' data analysis and, where the researcher has compared the data many times. The preliminary results were presented and discussed at a seminar ${ }^{8}$ attended by

${ }^{8}$ http://www.dagstuhl.de/de/programm/kalender/semhp/?semnr $=16162$ 
top researchers in this field. It is important to observe that when we infer that awareness of technical debt could impact software quality, we are describing the quality perceived by the team.

- Objectivity: The results show the information derived from the emerged data, codes and categories, related to the data quotes.

\subsection{Chapter Summary}

This chapter has discussed how the research was executed describing how we have conducted the case study in a two offering of the XP Lab, a classroom study, applied the interviews, questionnaires, and observations. Furthermore, we presented impacts on the team's behaviors once they started to consider technical debt. These impacts involve an improvement in the team communication. In the team member's opinion it also helps to improve the software quality. We also related others impacts on the teams due to being aware of technical debt. Also, we described and presented some approaches to help teams to identify, make visible and manage technical debt, such as technical debt board and card. Finally, we showed the analyzes of the code with the tools, SonarQube and Code Climate. 


\section{Chapter 5}

\section{Company A Studies}

Three studies were conducted at two different software development companies, operating in different business areas in the Information Technology (IT) market, Company A and Company $\mathrm{B}$,(fictitious name for confidentiality issues). In this chapter, we will discuss the two studies we conducted in Company A.

Below we described how the study was developed in each case, as well as the research design was applied and the results obtained.

\subsection{Context}

Company A is a large company in the IT market, which offers several online products. The company was founded in 1996 as an internet service provider focusing on content and dial-up services. Thenceforward it has diversified its range of products and services and evolved along with the technologies and practices adopted in the internet market [72].

In mid-2006, structural changes were made regarding how to manage the business and the products, which from that point on would be divided into business units. Given this new organizational form of the company and the launch of several products, it was observed that the software specification and development method became inefficient. The process was not adherent to the constant changes of products and continuous evolution, nor to the constant changes of requirements. The company scenario was distance from the business area, research and development teams(R\&D), which made it difficult to correctly specify the systems and thus generated a lot of effort from those involved in discussing the requirements. Another point is that the product development work lasted for months, without the business area seeing the result of the product. In view of the Company's situation and after several analyses it was concluded that Scrum [85] would be the best scenario for software development at the moment, causing some development teams to begin migrating to Scrum [103]. Since 2006 the company used Scrum, and thus, in 2012, they believed that they had a high degree of maturity in agile methods [103].

The study was conducted in the Research \& Development Department(R\&D) which is responsible for the design, development, and maintenance of all products and services provided by the company [73]. The company's development team consists of more than 150 developers divided into approximately 30 development teams, all of which use Scrum [73].

Two studies were carried out in this company, in different periods of time. The first, that we will here call Study 01, was carried out in 2012 and aimed at investigating whether technical debt had been incurred. We were also interested in understanding the motivations and impact of the technical debt items. In the second study, which we will call Study 02, our goal was to identify, monitor, and manage technical debt items in an agile software development project team without interfering in the software development process.

In the next sections, we will contextualize the step by step followed in the research process. We also describe how we analyzed the data and what were the results achieved in each study separately. 


\subsubsection{Study 01}

It was identified that, though the company had notion of the concept of technical debt, they did not consider it in practice at the moment of decision making and did not use it in project management. So, with the objective of collecting possible technical debt items, some milestones of changes in the project were defined through interviews with the team members. For each such milestone, we tried to identify if technical debt items were incurred in those moments. The identified technical debt items were analyzed in greater detail in order to understand the reasons that caused them and their impacts. This analysis resulted in a model that can be observed in Figure 5.4

In this study our goal was to answer the following questions:

- RQ1: Which decisions may motivate a team to incur technical debt in agile software development projects?

- RQ2: What types of technical debt items did the agile software development team incur?

- RQ3: What is the impact on an agile software development team of the technical debt in their project?

In order to answer these questions, we identify four cases where technical debt items were incurred. The four cases are contextualized below:

- Case 01 - Definition of the persistence form of monitoring events: the monitoring system developed was based on the Nagios framework 1 as a back-end platform. However, Nagios had architectural constraints that limited the scalability of the tool. There was a need to monitor a scale of thousands of devices and at these values, it was not possible to use only the back-end of the tool. As a way to optimize the Nagios performance, the developers opted to only persist the status changes of the monitoring and not the metrics collected by the monitoring agents.

- Case 02 - The development team made the CMDB (Configuration Management Database) mandatory for inserting information in the monitoring tool:: the development of the monitoring and automation tool was carried out in parallel with the implementation of ITIL (Information Technology Infrastructure Library) in the company [10]. A tool for problem management, changes, and configuration management (CMDB) was implemented. The monitoring tool needs a whole set of configuration components (CI) and relationships. These CIs are servers, racks, switches, ports of switches, routers, among others. The way to feed the tool was exclusively via CMDB integration

- Case 03 - Upgrade of the jQuery version: jQuery ${ }^{2}$ is a JavaScript cross-browser library developed to simplify client-side scripts that interact with HTML. jQuery is the most popular of the JavaScript libraries used in the world2. JQuery version 1.1 was used and with the evolution in the development and implementation of new features, several bugs and limitations were found in the library to support all the requirements. The interface technical team did some analyses and decided that by migrating to version 1.3 of jQuery the majority of the bugs would be fixed. In addition, it would be possible to implement new features to improve the system.

- Case 4 - Using MON 3 as a monitoring agent in the tool: MON is a component that works as an agent for monitoring availability of services and sending alerts exclusively on servers. The decision to use this agent was taken because the main deliverable of the project concerned the monitoring of servers and services with which the agent is compatible. It was a ready component (from the Open Source project), easy to maintain and configure, with a small learning curve for developing new monitoring plugins. 
Figure 5.1: Moment in which the Major Changes Occurred in the Cases Investigated.

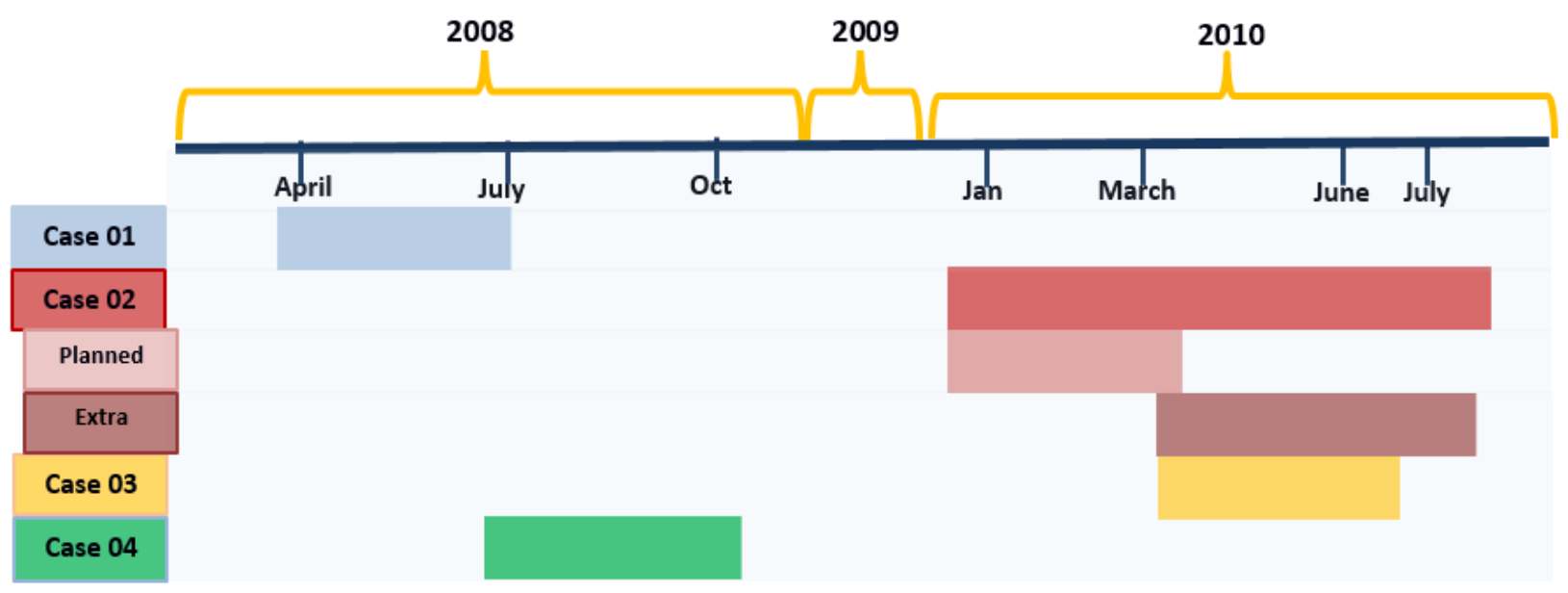

Source: [103]

In Figure 5.1 we can see the timeline showing when the major changes in each case occurred.

The major changes that were analyzed occurred between April 2008 and July 2010. The study was conducted at the company in 2012. In the next section, we describe the research steps, data analysis, and the results we found in the study 01.

\subsubsection{Research Design}

We followed six main steps in this study, as we can see in Figure 5.2 .

Figure 5.2: Research Steps on Study 01
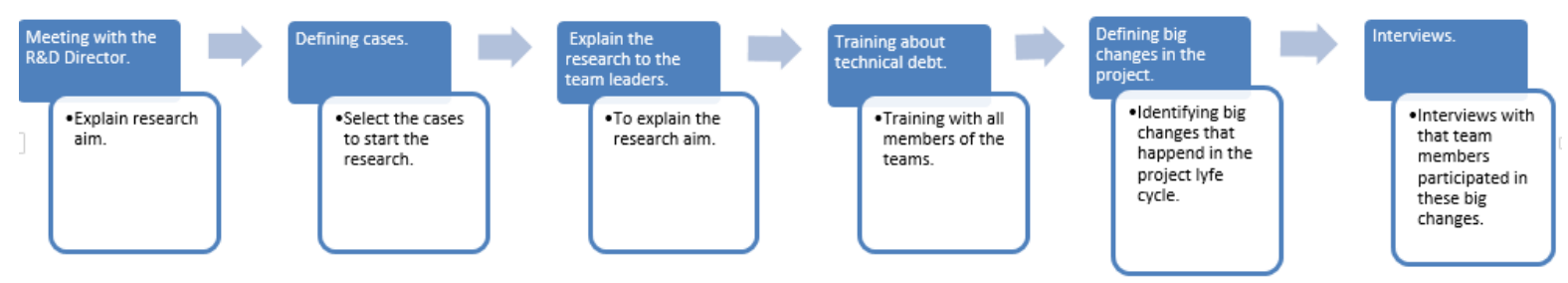

At first, a meeting was held with the company's R\&D director. In this first meeting we presented what we intended to investigate, as well as a brief discussion on the concept of technical debt. The $\mathrm{R} \& \mathrm{D}$ director and the two researchers interested in the study participated in this meeting. One of the researches was the Product Owner of the studied projects, but at the time was allocated to another sector of the same company. After the R\&D director became interested in the study, he analyzed some projects and provided us with 4 cases to be studied from one project. Subsequently we did an interview with the team member that had participated of the project from the beginning. We encouraged him to reflect on moments of major changes in the project, as recommended in the work of Tonin [102. Then, we defined milestones for major changes in the life-cycle of the identified cases, where some technical debt items could have been incurred. In order to do that we used the design suggestions in the Tonin study [102] as we can see in Figure 5.3.

\footnotetext{
${ }^{1}$ https://www.nagios.org/

2 http://trends.builtwith.com/javascript/jQuery

3 https://mon.wiki.kernel.org/
} 
Figure 5.3: Steps to Identify Big Changes

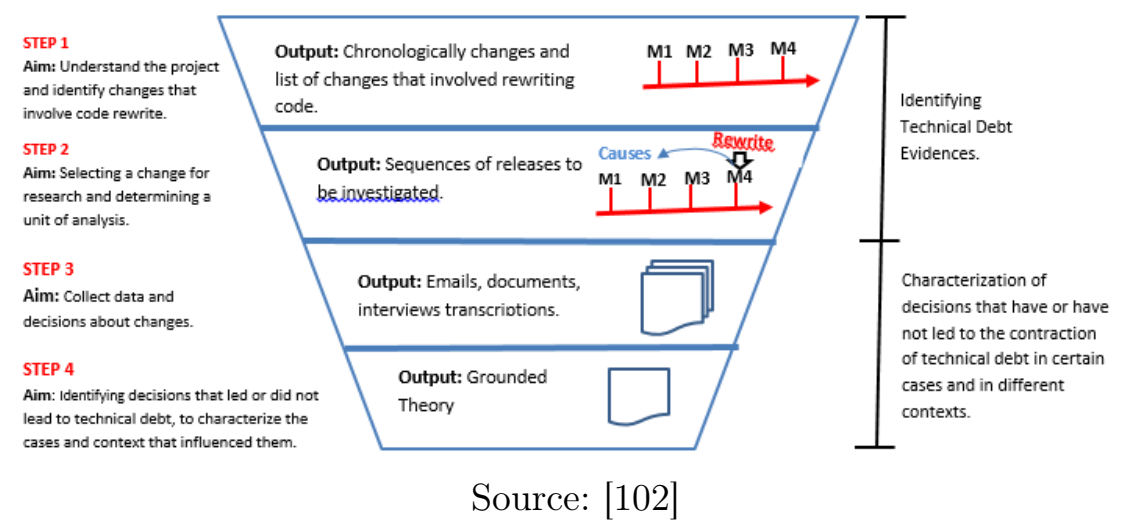

First we understood the projects and identified the big changes that involve code rewrite. Then, we organized these milestones in a chronological timeline and defined the releases that would be analyzed. After that, we proceeded to analyze each milestone and collect data through interviews, aiming to find evidence that showed how at that moment a technical debt item was probably incurred. After highlighting some milestones in the life-cycle of the project, individual interviews were conducted once with five team member's, of whom three were on the project from the outset. Three other people considered key at the time of decision making, no longer participated in the project and could not be interviewed. The focus of the interview was to identify the existence or not of the technical debt items in those moments of great changes, as well as possible motivations, strategic decisions that may have originated and their impact. We conduct a semi-structured interview following the Merriam recommendations [64], with open and closed questions. For it, we divided the interview into three steps: first, we explained the reasons for the research, second, we talked about some specifics moments we identified in the previous interview, and third, we asked the questions previously defined in a script. The questions had three main objectives:

- Characterize the role of the interviewee at that particular moment in the project. Identify what the interviewee remembered about that moment in the project.

- Specific questions about decisions made, problems faced and impacts generated, at that moment and throughout the evolution of the system in the interviewed.

- Some questions were added during the interviews if the researcher felt it necessary to make some information clear.

Data from the interviews were transcribed and analyzed using the steps of the Grounded Theory approach and we used the Nvivd ${ }^{4}$ tool for the qualitative data analysis.

This study was carried out by the researcher of this thesis and accompanied by the other researcher who assisted in some analysis of the data and review of the conclusions, as well as in the application of the interviews. In addition, the models were created by the researcher of this thesis and later discussed with the advisor and co-advisor and the other researcher.

As a result we create a model with the reasons of technical debt incurrence and impact, it is described in the next section.

\subsubsection{Results}

Figure 5.4 shows the model that presents the common categories and characteristics identified in each case studied.

In this model, it is possible to observe that all the technical debt items incurred were originated by a need for change, whether arising from system performance problems or from constraints of

${ }^{4}$ http://www.qsrinternational.com/products_nvivo.aspx 
Figure 5.4: Origin and Impact of the Technical Debt Items Studied

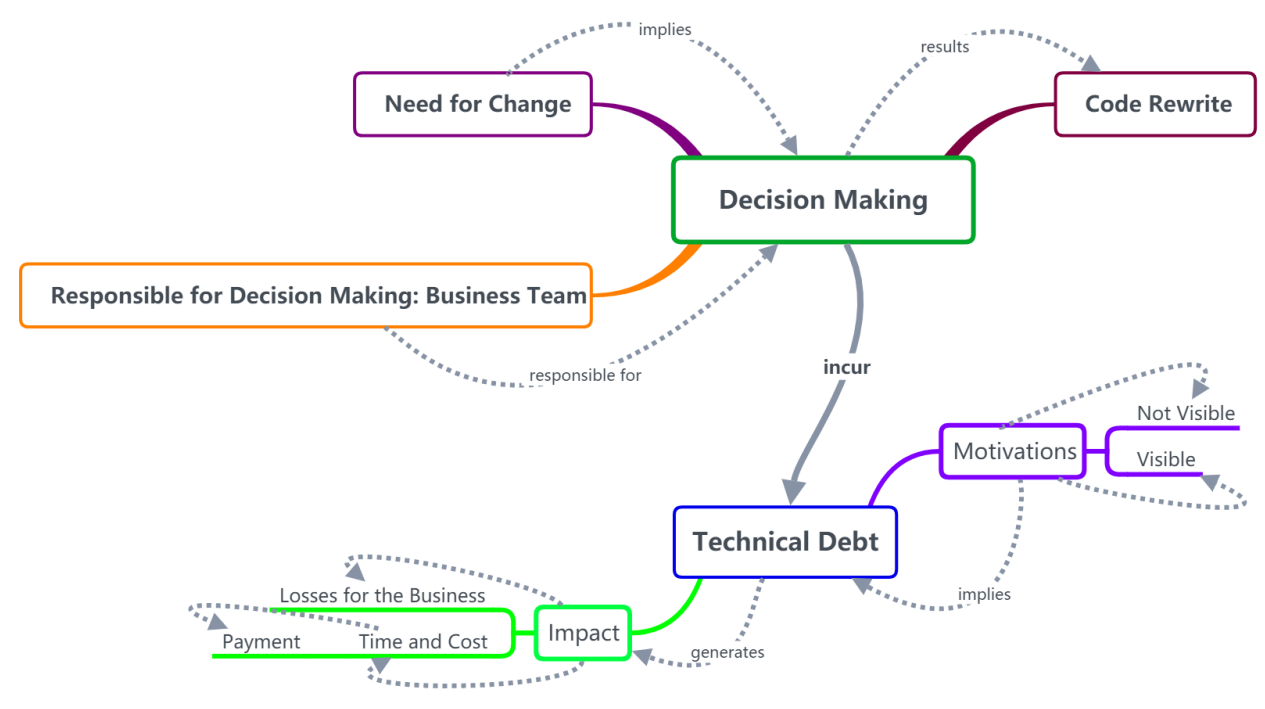

Source: [103]

the business. Often the business needed to adapt the way the tool/technology works to continue 'evolving'. After this need for change arises, the project team did not investigated several possible solutions to find the best one to solve that problem. And usually a member of the team with the best arguments and/or dominate a given technology, convinced decision-makers to follow a certain way. Normally, the final decision to implement a change came from a decision-maker (managers, directors, customer). These debts were incurred without the perception of most of the people involved in the project. As a result, it was necessary to rewrite code doing a refactoring to pay off the technical debt item at any given moment in the project, even if unconsciously. The motivation that originated the technical debt items may not often be visible to all involved, but the impacts commonly were. And the consequences of having them incurred in the cases studied negatively impacted the business, affecting time and cost. It also it results in negative impact for the business e.g can not attend the market evolution. In addition, in the Figure 5.5 it is possible to observe that in the cases studied, the technical debt items delayed the delivery of the project. The case Upgrade of the jQuery version was impacted in more than 120 days, and in the other three cases also the technical debt items impacted the project in over a month.

This model was used to categorize the cases studied. The result of the first case can be observed in Figure 5.6 .

Through the collected evidence, we identified that at the moment of decision-making of the Case 1 was incurred a technical debt that was categorized as follow:

- Decision-Making: the team did the upgrade of the jQuery Version.

- Influences on this Decision: team member with great technical knowledge convinced everyone that it would be quick to do and the gain would be great.

- Motivation:

1. Visible: could fix some bugs and gain in performance.

2. Non-Visible: technicians convinced that this was the best way to do it. First, they made an analysis based only on the benchmark document published on the tool's website; then, they decided to change the architecture of the entire front-end of the project, but they did not make it clear to the other team members nor to the customer.

- Impact: 
Figure 5.5: Effort Spent Due to the Incurrence of Technical Debt Items Per Project

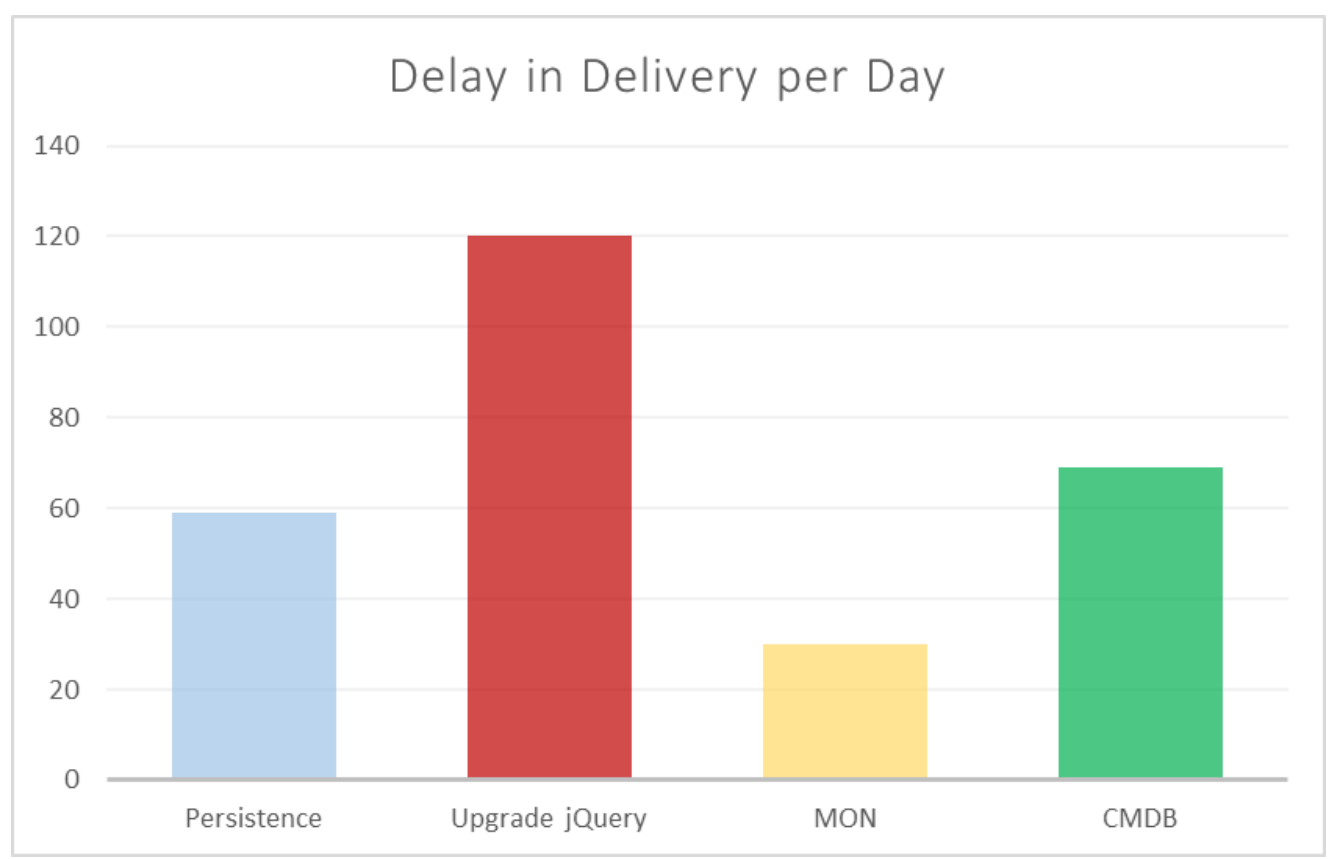

Source: [103]

Figure 5.6: Case 01 - Upgrade of the jQuery Version

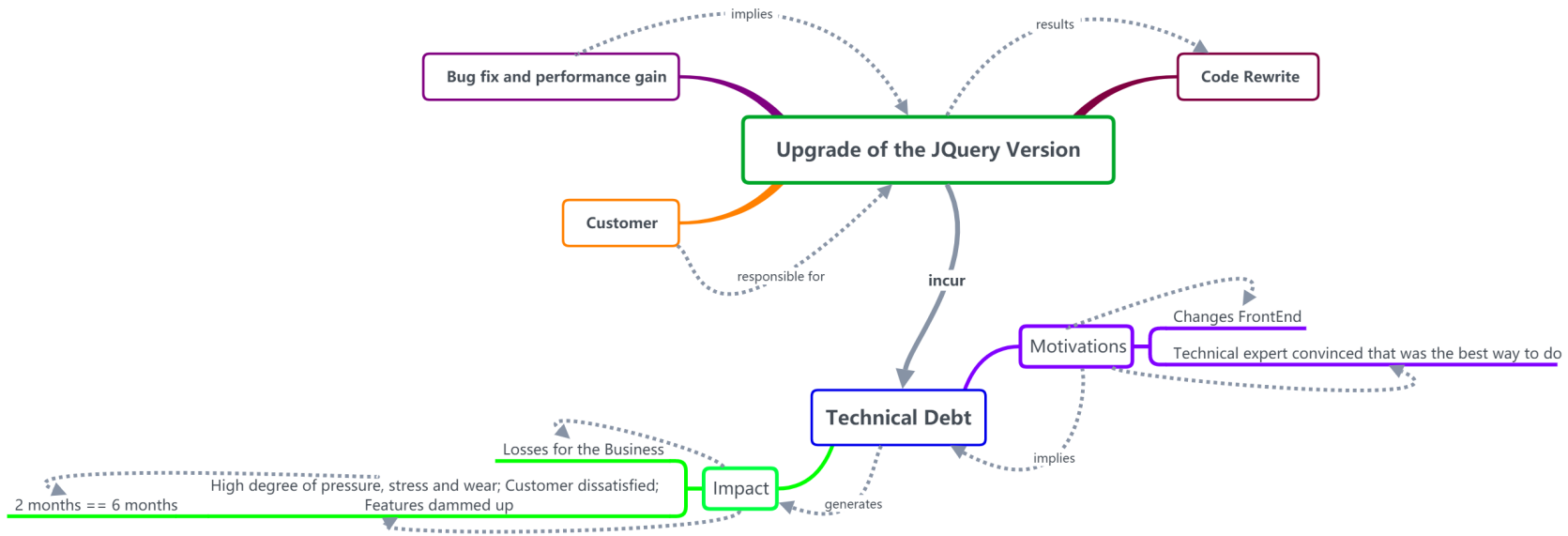

Source: [103]

1. Direct: the initial planned estimate was 2 months, but took more than 6 months; team under a high degree of pressure, stress and fatigue; customer demanding results.

2. Indirect: many other new features were developed and could not be placed in the production environment since they did not have a functional front-end due to the upgrade; customer dissatisfied.

The result of the second case can be observed in Figure 5.7

In Case 02, we identified that at the moment of the decision-making a technical debt was incurred.

- Decision-Making: definition of the persistence form of monitoring events in the database.

- Influences on this decision: team member points out as the best solution at the moment, since he had knowledge of such technology. 
Figure 5.7: Case 02 - Definition of The Persistence Form of Monitoring Events

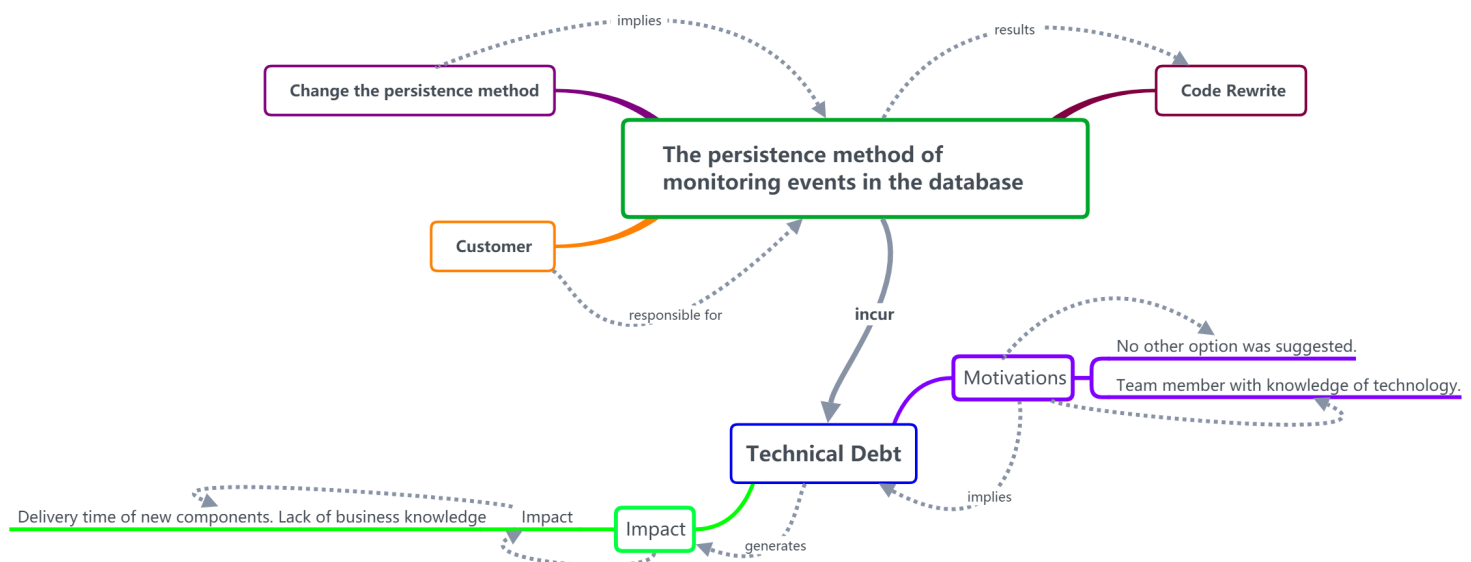

Source: [103]

- Motivation:

1. Visible: need to change how they did the persistence of monitoring events.

2. Non-Visible: only the professional who suggested using the tool knew it. Little or no search was performed to find another alternative.

- Impact:

1. Direct: delivery time of new components took longer than expected, since it was necessary to adapt the implemented technology.

2. Indirect: the lack of business strategy and vision on the future objectives of the business, such as: new customers. It may have influenced the team to make mistakes when choosing a certain technology, thus implying the need to change in the short term and limiting possible solutions that could be offered to the customer.

The result of the third case can be observed in Figure 5.8 .

Figure 5.8: Case 03 - Using MON as a Monitoring Agent in the Tool

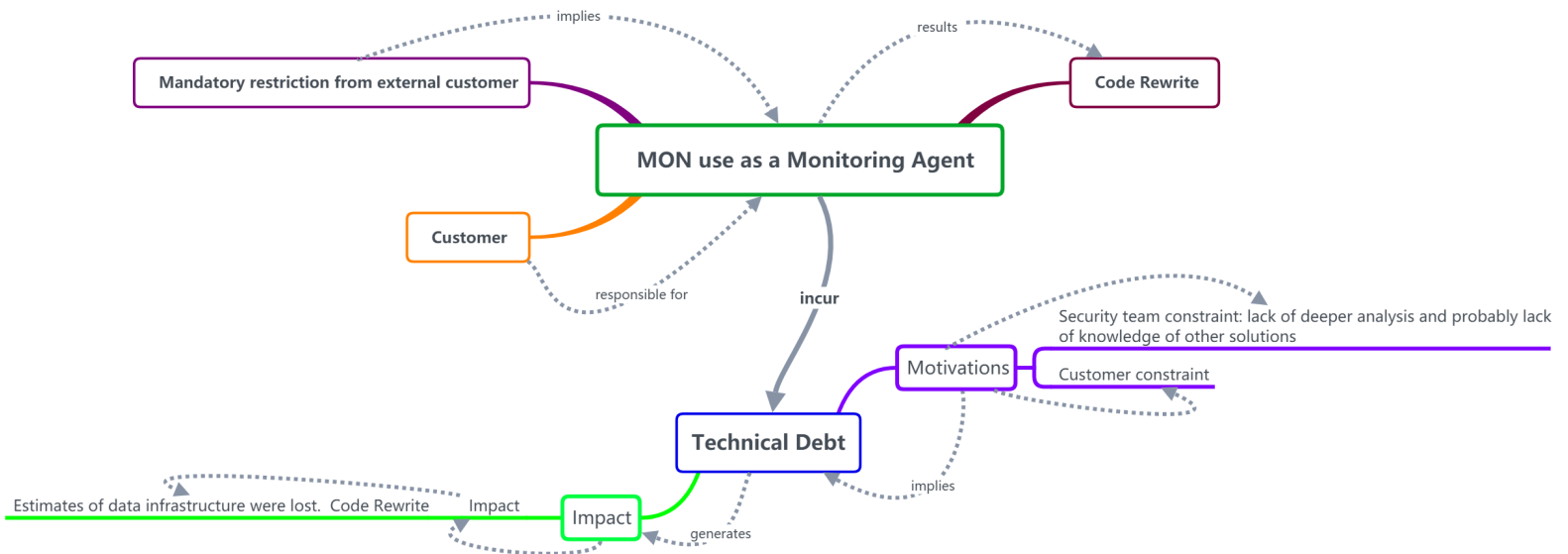

Source: [103]

We identified that at the moment of the decision making, a technical debt was incurred, which was categorized as follows: 
- Decision-Making: using $\mathrm{MON}^{5}$ as the monitoring agent in the tool.

- Influences of this decision: external customer, placed as a mandatory restriction did not use another alternative considered, which in this case was SNMP ${ }^{6}$.

- Motivation:

1. Visible: restriction imposed by the security team.

2. Non-Visible: nobody disagreed, probably because, on the part of the project team, they lacked technical knowledge to assert such an alternative. (Lack of a more in-depth analysis that took into account what competitive products were already available in the market).

- Impacts:

1. Direct: code rewriting, so new features had to adapt to the existing solution. However, at a given moment a new solution had to be acquired to solve the problem.

2. Indirect: many estimates of infrastructure data have been lost.

The result of the fourth case can be observed in Figure 5.9

Figure 5.9: Case 04 - The Configuration Management Database

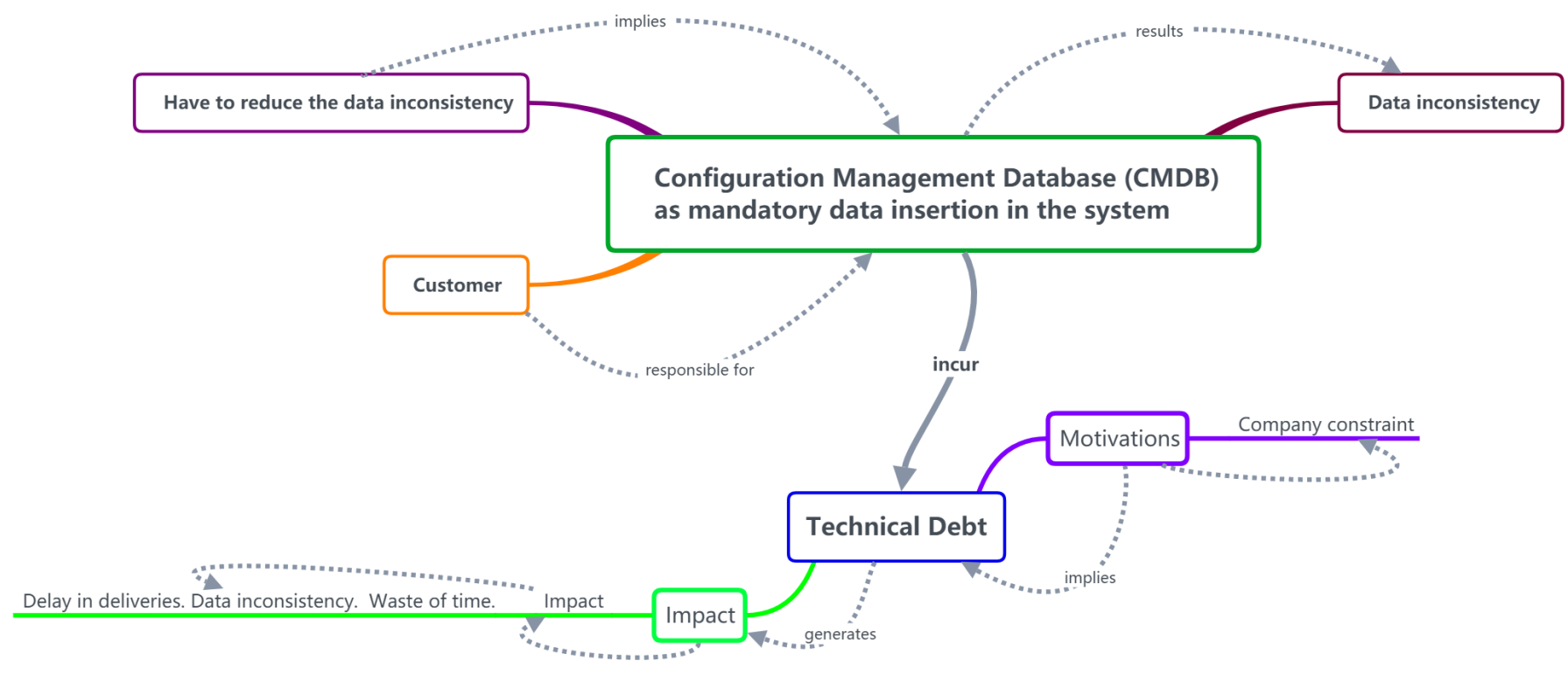

Source: [103]

We identified that at the moment of the decision making, a technical debt was incurred and categorized as follow:

- Decision-making: making the Configuration Management Database(CMDB) mandatory as the only way to insert information into the tool.

- Influences of this decision: mandatory of the Company's directors.

- Motivation: data scattered in various places and often inconsistent.

${ }^{5}$ https://mon.wiki.kernel.org/

${ }^{6} \mathrm{http}: / /$ www.snmplink.org/ 
- Impact:

1. Direct: many features were delayed because they depended on data coming from this system and these were not updated.

2. Indirect: sometimes what was pointed out as a bug of the system was in fact only inconsistency in the data coming from the CMDB and it took some time to reach a conclusion and/or prove this to the reporter of the bug.

This model reflects only impacts and motivations of the case studies and therefore can not be generalized, but can be easily applied and tested in other cases.

Additionally, we summarized the results of the cases and categorized them according to Cunningham properties, as we can observe in Figure 5.10, [20].

Figure 5.10: Technical Debt Categorization

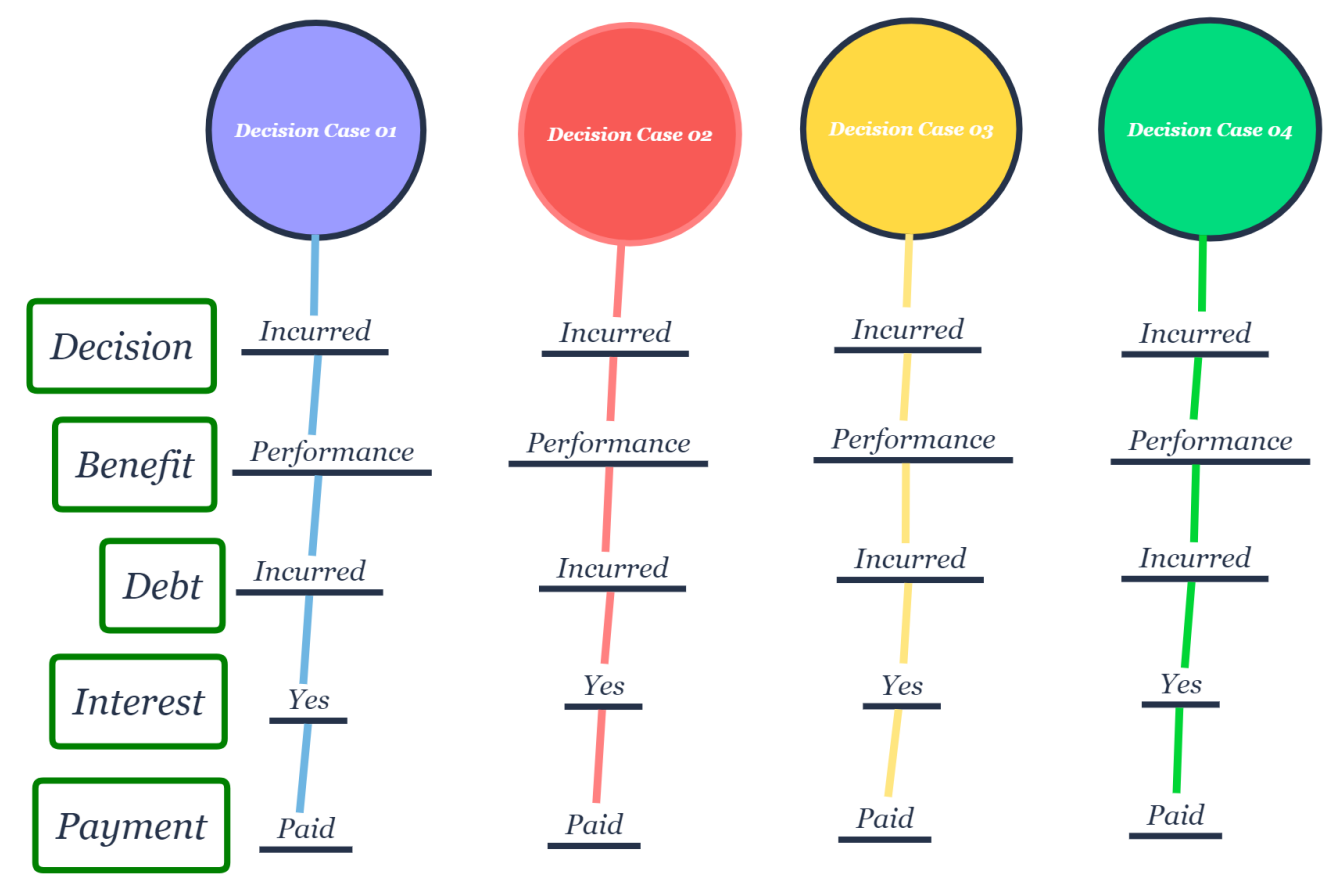

Source: [103]

In Figure 5.10 can be observed that all the decision-making incurred technical debt. . These decisions were made in order to improve the performance of some parts of the software and because of it, the technical debt resulted in interest, which had to be paid in the form of code rewriting, mainly impacting on the cost and time of the project.

In this study, we verified that it was important to identify and monitor the company's technical debt items because, over time, they could have a significant impact on the project. Considering this information, a second study was planned in the same company. This study will be explained below.

\subsubsection{Study 02}

The second study carried out in this company - we will call it Study 02 - targeted at suggesting an approach for technical debt identification and monitoring and verifying its effect, adapting to the management and development process followed by the team. The questions that guide this study were:

- RQ7: Which techniques and tools can agile software development teams use to identify, monitor and manage technical debt? 
- RQ8: What is the impact on the software development process once the agile software development team considers technical debt items?

Aiming to answer these questions, three projects were studied:

- Monitoring Project and Automation: this system works as a platform that is integrated with all the products developed in the company. Its main characteristics are the graphical monitoring of servers, automation of server delivery, automation of delivery, maintenance of software components in production, and access control of the companys production environment.

- Host Platform Design: this system consists of a sales server and provisioning platform for servers on the internet, in which the customer requests the equipment through a portal and the machine is provisioned and delivered based on customer requirements.

- Domain platform design: this system is responsible for all portal domains, sale and control. It is a very critical system that interacts with almost all system platforms in the company.

The steps we took to develop this study are described below.

\subsubsection{Research Design}

The following steps were conducted during this study, as we can see in Figure 5.11

Figure 5.11: Research Steps on Study 02.

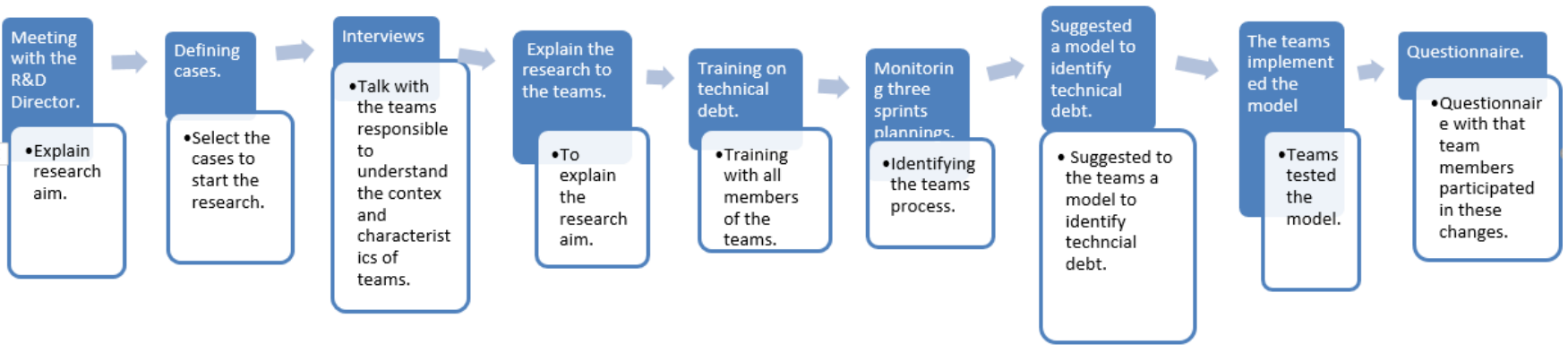

In this study, a meeting to explain the research was held with the RD director, who suggested three teams be studied. Therefore, the person responsible for each team was interviewed. In this interview, the aim was to understand the team and the project context. The interview had a semistructured format, with open and closed questions.

After the interview, two meetings were held with each team. The first meeting was held to explain the context of the research and at the second meeting a training on technical debt was conducted. After that, three sprint planning meetings of each team were followed to identify the work process of the teams, which tools were used to manage the sprints, and the development process. The sprint planning meeting was chosen because it is a planning meeting in which all participants in the project are assembled to discuss the next steps of the development process (members, scrum master, and product owner) [73. At these meetings, data were collected through notes and audio. The audios were transcribed and the data was analyzed. Through the analysis of the data collected in these meetings and based on the technical debt framework created by Seaman [87, a preliminary model of identification, monitoring, and management of technical debt was created and proposed. This template was presented to the team as a suggestion to be inserted in the team process.

The model was explained to the team members, who considered it interesting and decided to adopt it. 
After some sprints, in which the teams already used the model, one of the researchers made a questionnaire to identify the impact of the model according to the team's view. For this, the interview was based on questions like (the complete list is available in Appendix C):

- Does the identification and management of technical debt have any effect on software quality?

- What are the effects on product development of identifying and managing technical debt?

- Could you list the strengths and weaknesses of technical debt management through Jira?

- What is the impact of the technical debt management model on the team development process?

The steps of the Grounded Theory approach were used in the data analysis of this study.

It is important to highlight that the second study in Company A is part of a Master Degree Dissertation, hence it having been conducted by two researchers. The researcher of this thesis participated during the entire process. Most of the time, the data collection was done by only the other researcher, since he worked in the same company where the study was performed and the researcher of this thesis lived in another city. However, the researcher of this thesis participated in the planning of the study, the first meeting with the $R \& D$ director, and the technical debt meeting with the teams, helped the other researcher prepare the technical debt training, and participated in the two sprint planning meetings with some teams. In each meeting, both researchers discussed the next steps and actions to be taken, as well as the technical debt template. The first model of the interview was created by the researcher of the dissertation, and was later revised and adjusted by the researcher of this thesis. In addition, the diagrams resulting from the data analysis were created by the researcher of this thesis and re-analyzed by the other researcher.

\subsubsection{Results}

The purpose of this study was to identify the way of work of three company development teams, to suggest an approach for the technical debt identification and monitoring, and to verify its impact, adapting to the management and development process followed by the team. We first created and suggested a template so that teams would start considering technical debt, while bearing in mind the templates, tools and the process already used by the teams. Then we suggested a template of technical debt identification, monitoring and management. This model was further evaluated by questionnaire.

5.1.2.2.1 The Results of Data Analysis with Grounded Theory The data collected were analyzed according to the Grounded Theory technique. For data analysis, we used the NVivd 7 tool, which is widely used for analysis of qualitative data. In this case, the goal was not to use Grounded Theory to develop a theory but to simply use its steps to answer the research questions. The questionnaire was sent to 19 team members of the three teams studied, out of which 14 answered.

5.1.2.2.1.1 Open Coding In this first step, the data was interpreted to identify patterns and define codes, ". . . event/action/interaction, are compared against others for similarities and differences; they are also conceptually labeled (...) conceptually similar ones are grouped together to form categories and their subcategories. .." [19].

The codes resulting from the open coding were, Figure 5.12

5.1.2.2.1.2 Axial Coding In axial coding ". . . categories are related to their subcategories, and these relationships tested against the data. .." [19].

The resulting categories were as follows, Figure 5.13 .

\footnotetext{
7http://www.qsrinternational.com/support/downloads/nvivo-9
} 
Figure 5.12: Categories resulting from the open coding.

\begin{tabular}{|c|c|}
\hline Codes & What They Talk About \\
\hline Technical Debt Management & $\begin{array}{l}\text { "...the technical debt identification and } \\
\text { management helped the team members to identify } \\
\text { parts of softwa re to improve that may facilitate the } \\
\text { maintenance and evolution of software ..." }\end{array}$ \\
\hline Technical Debt Visibility & $\begin{array}{l}\text { "...easy visualization of the type technical debt } \\
\text { stories ..." }\end{array}$ \\
\hline Story Management & $\begin{array}{l}\text { "... separated and dedicated stories for the } \\
\text { treatment of technical debt items ..." }\end{array}$ \\
\hline Planning & $\begin{array}{l}\text { "...the technical debt prioritization and } \\
\text { management...being an important input to help in } \\
\text { prioritizing the backlog ..." }\end{array}$ \\
\hline P.O Negotiation & $\begin{array}{l}\text { "... became clearer to the team and to the P.O what } \\
\text { are improvement stories and what are technical debt } \\
\text { stories ..." }\end{array}$ \\
\hline Technical Debt Payment & $\begin{array}{l}\text { "...some hours of the team are destined to pay the } \\
\text { technical debt items..." }\end{array}$ \\
\hline Awareness & $\begin{array}{l}\text { "..the team shows to the P.O the technical debt } \\
\text { items..." }\end{array}$ \\
\hline Documentation & “...easy access to the stories' documentation..." \\
\hline Quality Improvement & $\begin{array}{l}\text { "...it is possible to make explicit problems for the } \\
\text { software and to identify critical points in system } \\
\text { quality in a supported way..." }\end{array}$ \\
\hline Technical Debt Cost & $\begin{array}{l}\text { "...technical debt stories were prioritized according } \\
\text { to cost in conjunction with regular stories..." }\end{array}$ \\
\hline Refactoring & $\begin{array}{l}\text { "...hel ping to prioritize and correct problems, which } \\
\text { optimizes to have a better system quality..." }\end{array}$ \\
\hline Time & $\begin{array}{l}\text { "...always trying to pay technical debt items as } \\
\text { quickly as possible..." }\end{array}$ \\
\hline Technical Debt Categorization & $\begin{array}{l}\text { "... At the time of the creation of the story it is } \\
\text { possible to easily categorize it as a technical debt, } \\
\text { with only a few fields filled ...". }\end{array}$ \\
\hline
\end{tabular}

\section{Source: 72}

Figure 5.13: Categories Resulting from the Evaluation of the Conceptual Model for Technical Debt Management

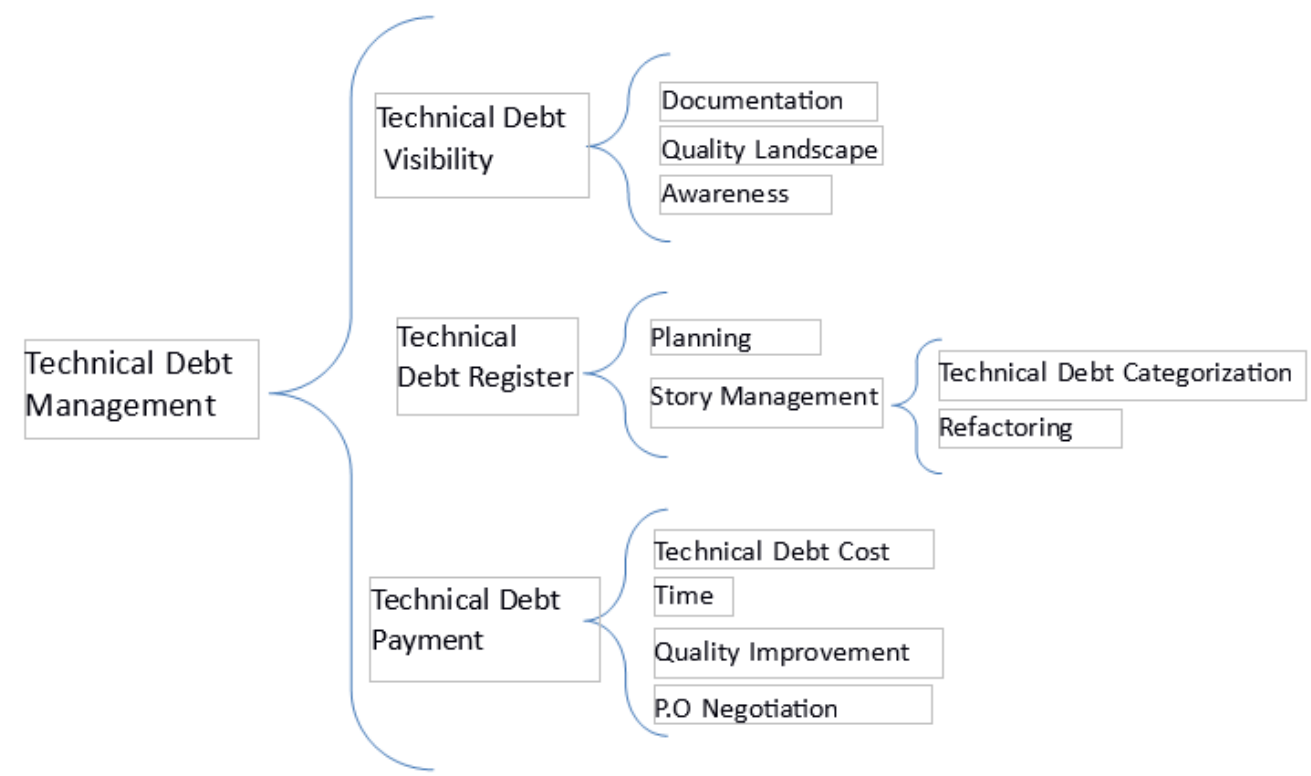

Source: Adapted from [72]

It was identified in the data analysis that for all teams it was evident that implementing the conceptual model for technical debt management made the teams become aware of the existing technical debt items in the software, since the identification and registration of the same generated greater visibility, "... now the team exposes to the P.O the technical debt items that exist in the software and these stories are executed by the team along with the stories of improvement and evolution of the product ...", [72. This visibility of the technical debt items provides a quality 
landscape, so the teams could see the number of the technical debt items they had inserted on the code. In addition, the technical debt list it is a complementary documentation for the software disabilities. Also, once they identify and register technical debt they could plan whether they will pay or not the technical debt items. Further, the model facilitated the technical debt story management, supporting the team to identify the technical debt cost. This way, they could categorize it according to the technical debt types predefined by the team, estimate and plan the technical debt payment [72 by refactoring. Being aware of the technical debt items and keeping them visible can help at the time of negotiation with P.O so that they can prioritize some items to be paid in the sprint. Then, they could set aside time to do the refactoring of that part of the code affected by the technical debt.

In addition, it provides the team with one more metric to improve the software quality, "... It is possible to make explicit problem of the software and identify critical points in the quality of the system in a supported way, helping to prioritize and correct problems, which optimizes the search for better quality in the system ...", [72].

5.1.2.2.1.3 Selective Coding In selective coding "... all categories are unified around a central 'core' category and categories that need further explanation are filled-in with descriptive details..." [19].

We also analyze the resulting categories and their relationship with the codes, as we can see in the Figure 5.14 .

Figure 5.14: Relationships between Categories and Codes

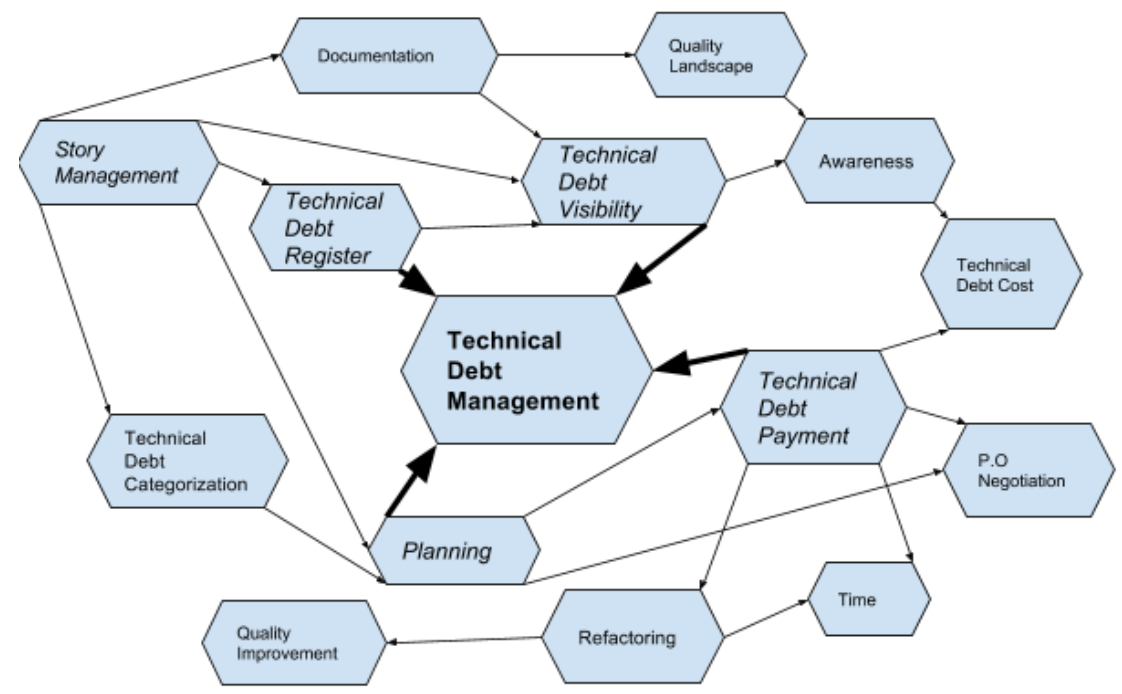

Source: Adapted from [72]

It is possible to observe that the technical debt register, visibility, payment and planning impact the technical debt management. So, the first version of the model for technical debt management helped the teams to manage technical debt items. The resulting main category is technical debt management. With this first version of the model for technical debt management, it is possible to control the technical debt items in a simple and easy way, using the same tools already known to the team and maintaining the same process.

5.1.2.2.2 The Proposed Template According to the studies of Seaman [87, in order to manage technical debt, it is necessary to identify it, monitor and estimate before one can begin to take the first steps in the technical debt management. We suggested some steps and ways of doing it.

This proposal suggested some modifications in the template of the task management tool already used by the team - in this case, it was the Jira 8 tool. Also, we suggested some changes in the form

$\sqrt[8]{\text { https://www.atlassian.com/software/jira }}$ 
they used to manage the sprints in order to identify and control technical debt in the story panel created and controlled by the teams 73 . Regarding the registration of technical debt items, some minor changes were made in the parameterization of the model already in use to register the stories [73.

In the identification of technical debt phase, a template to register the technical debt items in the tool of project management was adapted through a model already used for the stories register. It can be observed in Figures 5.15 and Figure 5.16 .

Figure 5.15: Type of Story Technical Debt

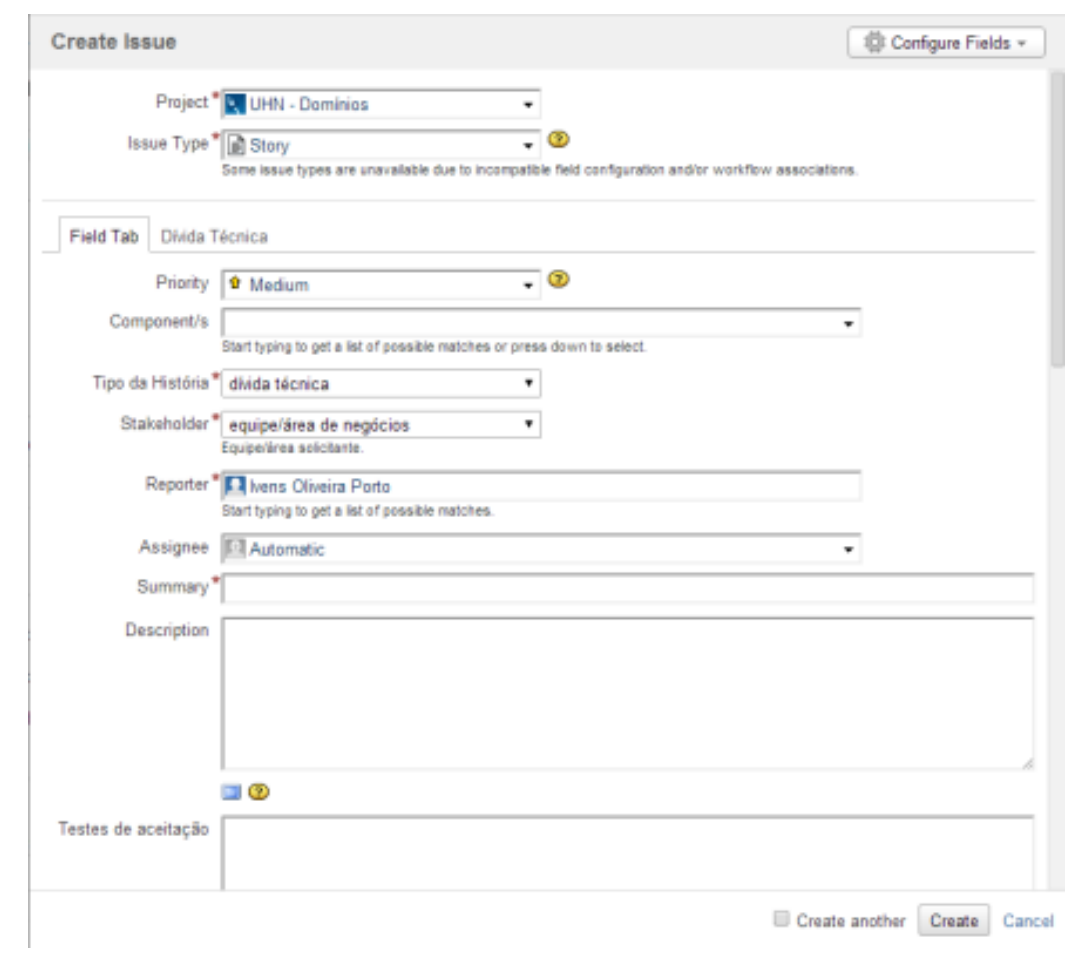

Source: 73

The story register has a field called History Type, where all stories recorded in the tool can be categorized into the following types: improvement, software defect and adjustment, (These categories were defined with researchers and team members, commonly suggested by team members). A technical debt type was added as can be seen in Figure 5.15 .

In addition to the story type,the option to select the type of technical debt being registered was created. Initially, the following types were created: architecture, defects, and test, as can be seen in Figure 5.16 (These types were defined with researchers and team members.).

Furthermore, int the technical debt form, as we can see in the Figure 5.17, we created a field to add the value of the technical debt interest, and the probability of the technical debt resulting in interest. The field interest could be filled with the amount of the points the team used to correct or maintain the technical debt item, and the probability could be filled with the measure: low, medium and high. Also they could fill in the following items: name, description, responsible for register the item, priority, related tests, stakeholder and who assigned to pay the debt.

Moreover, the technical debt items identified were estimated at the sprint planning meetings or in the estimation meeting. These were estimated and prioritized by the Product Owner (P.O.), too. The participants of the meeting discussed the technical debt item criticality and the impact on the project. If they believed that the item was relevant, it would be then added to the sprint backlog to be paid [73].

The technical debt items were visible in the team planning board along with the stories, as can be seen in Figure 5.18 . 
Figure 5.16: Type of Technical Debt Items

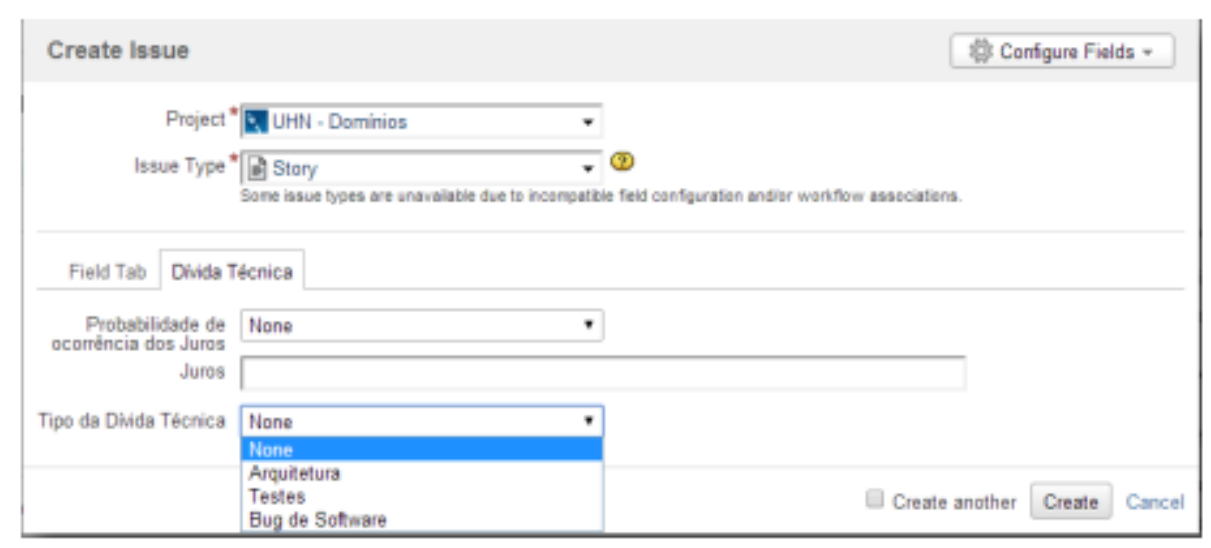

Source: [72]

Figure 5.17: Technical Debt Item

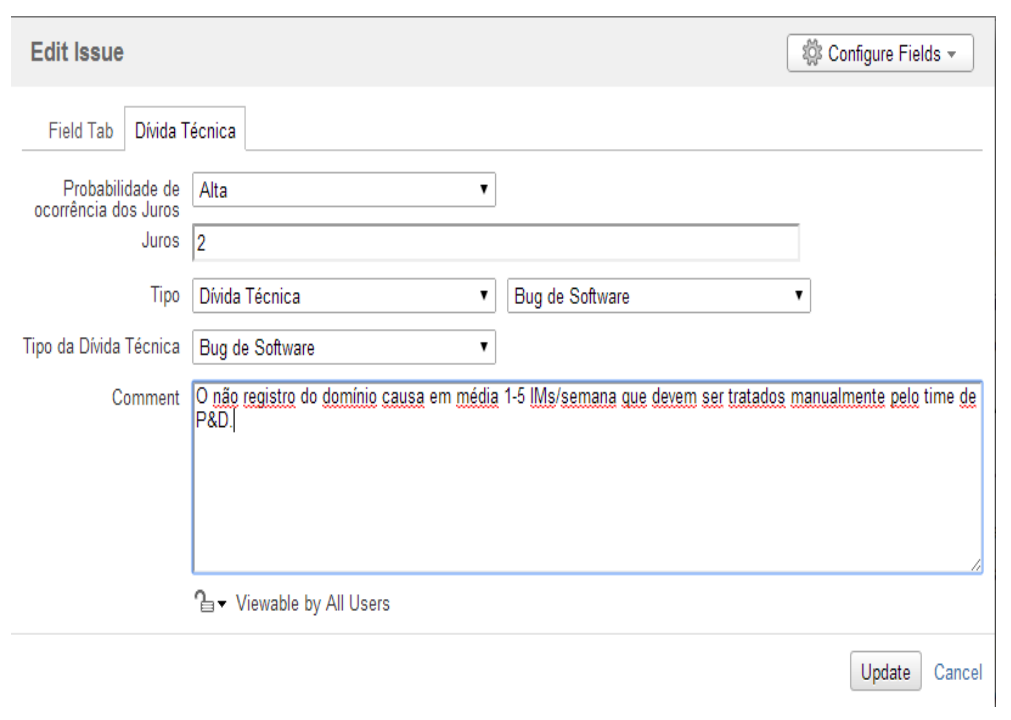

Source: [72]

5.1.2.2.3 The Conceptual Model for Technical Debt Management As a main results of the studies in Company A we created a conceptual model, as we can see in Figure 5.19

In this conceptual model, it is possible to observe that when the teams decide to contract a technical debt item it is important to monitor the impact, the causes and also start managing it. All technical debt items are caused as a result of motivations such as: business decision, technology update, need for change, system performance, etc. Identifying this motivation helps the teams to mitigate and to plan how to deal with new technical debt items. Furthermore, these technical debt items result in some impacts on the project, as most of them incur time and cost, and can directly affect the business. Therefore, once technical debt is incurred it is necessary to manage it. For this, the team needs to define a template for technical debt identification. In addition, they have to select ways to maintain the technical debt list visible, for which they can use: boards, tools such as Jira, and meetings such as sprint planning. To decide about payment, they have to measure this list of technical debt items. This decision can be supported by discussing it with the team members in a specific meeting, such as sprint planning, when the team can analyze the context and the demand of that sprint, then decide if it is necessary to pay any technical debt item. The payment of the technical debt items will involve refactoring in the software code.

This model is the first step to create a robust model for technical debt management. In this Company A, the findings of the first study provide a landscape about where the debts can be 
Figure 5.18: Board of Tasks with Technical Debt Items

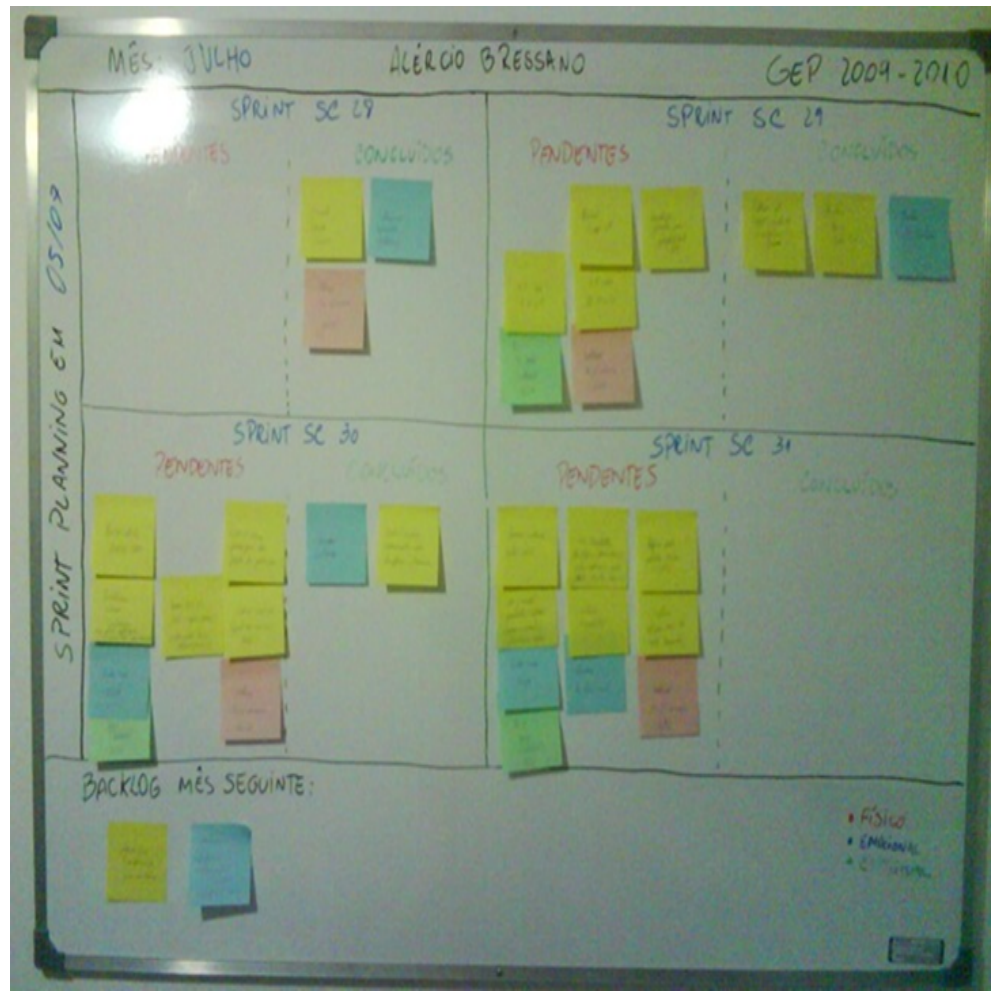

Source: 73

Figure 5.19: Main Results of Company A

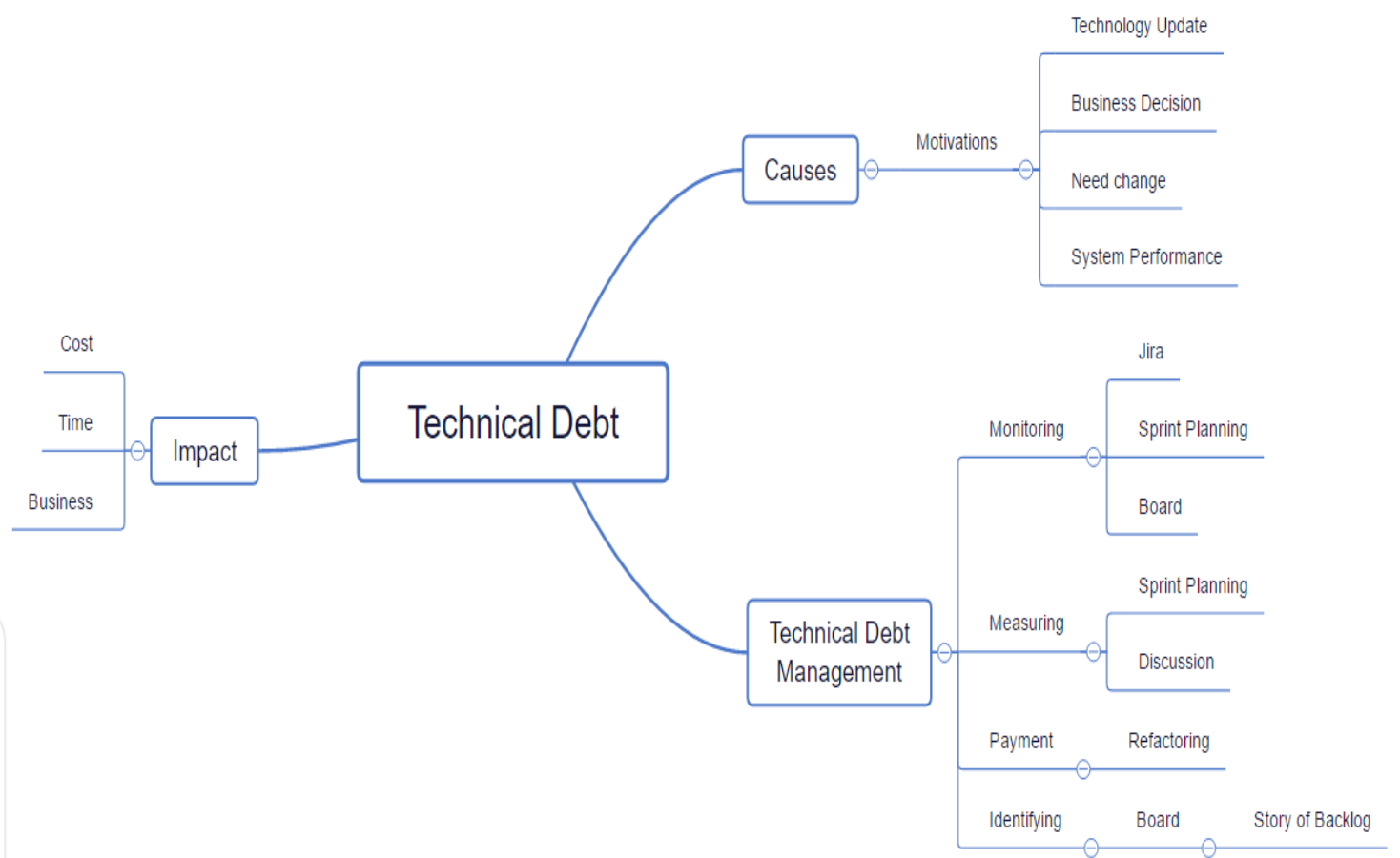

originated - which type of decision - also help the teams understand that consider technical debt is important to manage the software quality and complexity. Once they show significant impacts on the project, team and business, directly resulting in waste time, money, and affecting the relationship with to the customers. As a result of this first study, the teams understood the relevance of making the technical debt visible and manage them. So, they decided to do it, following some approaches suggested in our model. In conclusion, they tested the model resulting in the first version of the 
conceptual model for technical debt management. This first version of the conceptual model for technical debt management will be complemented in the next chapter with the findings of the Classroom and Companies studies. Then, the final conceptual model for technical debt management will be present, discussed and compared with the models found in the literature review.

\subsubsection{Threats to the Validity}

In this study, we took some actions to mitigate the bias. We describe these actions in the following points:

- Bias due to data analysis and interpretation: data were interpreted following the Grounded Theory steps. We analyzed the data separately, first the interviews transcript, followed by the answers of the questionnaires. We then compared and merged the findings that were relevant and offered plenty of evidence to the results of both studies. If we had any doubt about a specific point we went back to the data and re-analyzed them. After that, the results were read by the advisor and co-advisor and if they indicated some points to be re-analyzed, the researcher re-analyzed them. We did analysis and re-analysis many times until we were sure of the conclusions. We recommend that these studies be replicated in other cases and the results be compared before they can be generalized.

- Bias due to the context: in addition, it should be emphasized that the studies conducted in companies are aimed at solving the problems of the company and are the best option for those very specific cases. Therefore, the studies cannot be generalized either. We sought to develop them with the highest possible scientific rigor and we also sought to report the studies and the motivations of each decision in details so that they can be replicated and tested in other cases.

- Bias due to the software quality improvement: this study is premised on the assumption that once the teams started to consider technical debt, the software quality improved. This conclusion is based on the analysis of the data from team members' testimonials. Nonetheless, in the company studies we did not compare these beliefs with the analysis of the code quality.

\subsection{Chapter Summary}

This chapter has presented the studies we did in Company A, where we conducted two studies in different teams. We explain how we applied the method to the case study in Company A. First, we present the findings of the study 01 at Company A. In this study, we present the model to help teams identify causes, motivations, reasons and impact of the technical debt items. Then, we demonstrate how we did the second study. For data analysis, we used the steps of the Grounded Theory approach. We proposed some approaches for teams to use in technical debt management. We have recorded the technical debt items in the Scrum board, technical debt board and in the Jira tool. We use to monitor the debts the tools: SonarQube and Code climate. As a result, we show the first version of the conceptual model for technical debt management and the resulting analysis of this model according to the teams' perception. This model can be a simple guide to the teams that want to start identify and manage technical debt. 


\section{Chapter 6}

\section{Company B Studies}

Three action research cycles were conducted with two teams in this technology company, Company B (fictional name for confidentiality issues). Our goal was to identify, monitor and manage technical debt items in an agile software development project team.

\subsection{Context}

Company B develops products and services for financial institutions. The company in general has solid experience in the financial IT market, both domestic and international, and a highly experienced team in this area, which is a big advantage. The company's products are focused on risk management, qualified custody, back-office management systems, treasury, and payment systems [104].

The company has approximately 250 professionals from: Finance, Mathematics and Information Technology. About 200 professionals work in software development. Company B has more than 50 customers and seeks excellence in its solutions by using the best practices of innovation in the financial and technological markets [104].

In 2006, the company started using the agile Scrum framework in some teams. After realizing that this methodology is in line with the company's values of adding customer value, quality, technical excellence, people appreciation and organizational culture, Scrum has become the software development methodology of the company. When the study took place there were more than seven teams using Scrum. However, their level of adoption differed from team to team, according to their context and characteristics, such as level of involvement with the customer, team size, product domain, etc. There was an effort to follow the principles of the Agile Manifesto as a way to properly implement Scrum [104]. The studies were carried out in different environments with a great diversity of professional profiles. Whereas some undergraduate and graduate students were already allocated to the company, many of them lacked experience in the market.

The company's products were developed on the basis of the framework called Jmine, developed by their software developers to leverage the company products. Immature code, such as coupled code, high complexity, duplicate code and missing tests are the common problems they identify in the submitted pull requestshttp://help.collab.net/index.jsp?topic=/teamforge163/faq/pullrequest. html. Jmine is used by the products developed in the company and developed by the company team's. There is a team responsible for analyzing and applying such changes in the Jmine framework. Several teams sending changes concomitantly to the Jmine, and some merge inserted technical debt in the code. The framework is frequently improved by all developers of the company by submitting pull requests. Consequently, many technical debt items were incorporated to the framework throughout the products development [104].

In this company, there were at least two teams worried about how to deal with the technical debt items in their projects. Frequently they asked: "How can we identify technical debt items?", "How do we manage the technical debt items?", "How will we pay and prevent technical debt items?". With this in mind, the questions that guided this study were: 
- RQ4: Which techniques and tools can agile software development teams use to identify, monitor and manage technical debt?

- RQ5: What is the impact on the software development process when the agile software development teams considers technical debt items?

- RQ6: What types of technical debt items do the agile software development teams incur?

For the purpose of this study, we shall name the teams Team A and Team B. The teams had different concerns about technical debt and some common needs, such as visibility and monitoring of technical debt items [104. A description of each team is presented below.

\subsubsection{Team A}

Team A was responsible for managing the company base framework (Jmine) by analyzing issues submitted on the pull request, request that affect code quality [104]. The team A was composed by three developers. They are responsible for controlling the software quality of the company framework.

\subsubsection{Team B}

Team B was responsible for some of the company's products. They commonly worked under a tight schedule and a high delivery rate (product versions). The team often prioritized delivery features over code quality, resulting in poor code quality, lack of automated testing, and general dissatisfaction with product quality. In addition, this team did not have a well-defined development process, not only because of the lack of time but also because of political and cultural problems (lack of interest in the process, especially lack of knowledge of the real value of an agile process), [104.

\subsubsection{Research Design}

In the cases studied in Company B, the main objective was to create a technical debt management model. To begin the studies in the company, some meetings were held first with managers and then with technical leaders. In these meetings, the research objectives and the technical debt metaphor were presented.

Soon after, the company made its work environment available for the research and meetings with a technical leader were held in order to identify which projects could be studied. Once two projects were identified, meetings were held with the members of both teams. One of the teams was in charge of the treasury project and the other team was responsible for the quality of the base framework for all other projects, Jmine.

In this company, we use the action research method and three cycles of action research were performed, as can be seen in Figure 6.1.

In the first cycle, the objective was to discuss the concept of technical debt, as well as to introduce the first forms of technical debt identification. In the second cycle, the objective was to improve the forms of identification, to observe more types of technical debt items and then to start monitoring them. In the third cycle, it was possible to have a pattern of identification and to include more ways and tools to monitor technical debt items. As a result, according to the team's perception, considering technical debt has not only brought some benefits but as also made some problems emerge, such as those related to the team's motivation.

In the subsections below we will show how each cycle was conducted in each team.

\subsubsection{Team A}

In the Jmine team, three cycles were executed and the process that we followed is detailed below: 
Figure 6.1: Action Research Cycles by Team.

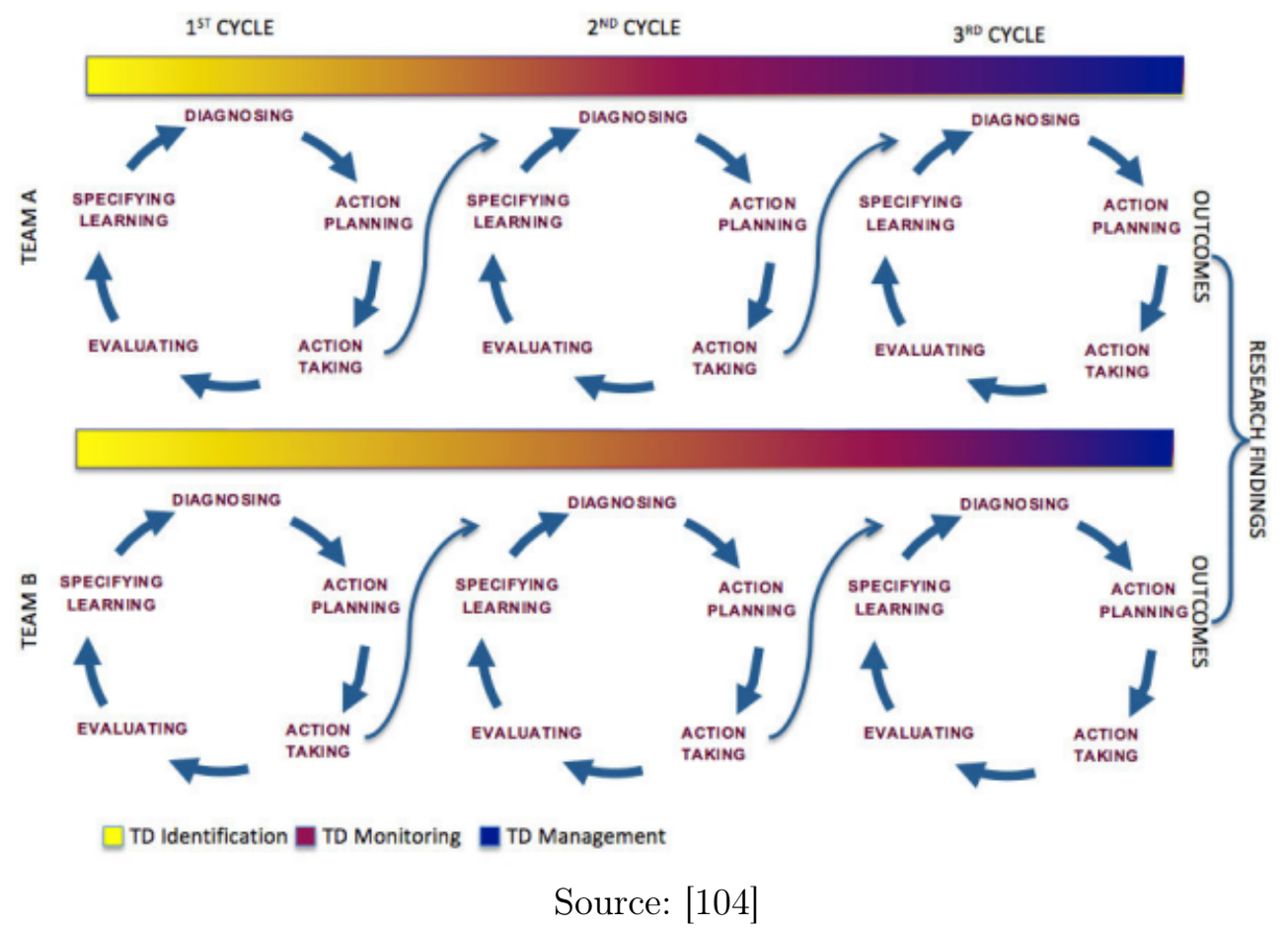

We conducted three cycle of the Action Research method, in this method the participants and researchers are involved cooperatively, seeking to implement certain actions to solve certain problems [58], we follow the five steps of action research method [5].

6.1.3.1.1 First Cycle In the first cycle, the objective was to start activities aimed at identifying the technical debt items.

A. Diagnosis: this team was aware that several technical debt items had been entered into the Jmine in each pull request performed by other teams. However, the team did not know what types of technical debt items were inserted, the severity and the location of them. The team's first concern of the team was to provide visibility of the technical debt items in the framework. So, the team began to study tools that could help to achieve this goal. Another concern was the large number of merges coming from other branches managed by other teams. There were lots of issues with urgent pull-request required, with no tests and/or bad design that needed to be visible to the team.

B. Action Plan: first, the members of this team held a meeting with the leaders of the teams making pull requests in Jmine. At this meeting it was decided that these members would discuss with their teams a plan that would be followed every time they made a commit. In addition, Team A decided start to study the SonarQube platform, which is a platform to manage quality and has a specific plugin to measure technical debt. Additionally, they planned to insert a new field in the project management tool -Jira - to registered the technical debt items. Another goal at that time was to disseminate the concept of technical debt with all Team A members.

C. Plan Application: a new field was created in the Jira tool that they used to manage the project. The field was called technical debt. After a first analysis of the SonarQube platform, the team concluded that it seemed to meet the demand. With the tool was possible to evaluate important factors such as: test coverage, complexity, possible bugs, architecture and design. One member of the Jmine team was choose for doing a more detailed analysis of SonarQube. The tool was installed on the member's computer so it could be tested. 
D. Evaluation: After testing the SonarQube platform, a team member presented the technical debt items indicated by the platform in the daily meetings. And, in a team retrospective meeting the team discussed and approved the installation of the platform on the production server. A plugin of the tool was installed on the Jenkinshttps://jenkins.io// server to trigger a parsing in the code after the daily building.

E. Learning/Evolution: the first landscape of the technical debt items provided by SonarQube helped Team A to identify which teams inserted technical debt items into the main code most often. One member of the team said: "... This allowed us to visualize which teams were inserting more technical debt items into the Jmine...". This visibility allowed them to identify the location of the technical debt items and so the team could define points of attention, prioritize technical debt items by severity and present the report to the responsible team as a way to raise awareness of the importance of the concept of technical debt. The SonarQube platform allowed them to anticipate potential problems.

6.1.3.1.2 Second Cycle The objective of this cycle was to focus on monitoring technical debt items.

A. Diagnostics: Team A had the need to monitor and be able to track technical debt items. For this particular case, it was not possible to track them with the SonarQube platform. Much of the pull request code had low quality. Many technical debt items were inserted in the Jmine code because they could not identify the problems before the pull requests. So it was necessary to find ways and approaches to monitor the technical debt items and anticipate problematic pull requests.

B. Action Plan: After the team analyzed some pull-analysis tools, Team A considered that there was no tool that could provide the support that they needed. They then started to develop a plugin to parse the pull request and automated the pull request parsing process. The team decided to create a report for the TV screen (They show the graphics of the projects in TV monitors in the development room), indicating which team had inserted more technical debt items into the Jmine code.

C. Plan Application: The project management tool was configured with a trigger, a parameter in the Jenkins tool that ran the pull requests analysis tool every time a pull request was submitted. This plugin was developed by the team responsible for building, analyzing and measuring the number of changes caused by pull requests that influence code quality. This plugin used other code quality analysis tools: Checkstyle 4http://checkstyle.sourceforge.net/, Coveragehttps:// cobertura.github.io/cobertura/, and PMDhttp://pmd.sourceforge.net/pmd-4.3.0/integrations. html, as can be seen in Figure 6.2. It is important to note that not all items resulting from the reports were technical debt items, so the reports went through the analysis of the quality team before adding such items as technical debt.

Team A began to register technical debt items and to identify teams that incurred them. The graphs that appear on the TV screen display information regarding the number of technical debt items incurred (per time, per product and per project). The graphs also contained the status of the builds.

A reliability metric for pull requests was created and it consisted of three values: low, medium, and high. When the reliability was low the pull requests was rejected and the requester was automatically notified. When reliability was medium a manual analysis was performed and if reliability was high the merge could be done automatically.

D. Evaluation: This automatic pull request analysis assisted not only Team A, but also all other teams that used the Jmine framework. Reports of the code quality and reliability level allowed Team A to spend their time helping other teams deal with their technical debt items. 
Figure 6.2: Graph Generated by the Pull Requests Analysis Tool

\begin{tabular}{|c|c|c|}
\hline \multirow[t]{2}{*}{ PMD report } & & \multirow[b]{2}{*}{ Problem } \\
\hline & Line & \\
\hline 1 /home/vinicius/workspace/dbmonitor/dbmonitor-base/src/main/java & 67 & These nested if statements could be combined \\
\hline 2 /home/vinicius/workspace/dbmonitor/dbmonitor-base/src/main/java & 139 & These nested if statements could be combined \\
\hline 3 /home/vinicius/workspace/dbmonitor/dbmonitor-base/src/main/java & 131 & Avoid empty catch blocks \\
\hline 4 /home/vinicius/workspace/dbmonitor/dbmonitor-base/src/main/java & 32 & Ensure you override both equals() and hashCode() \\
\hline 5 /home/vinicius/workspace/dbmonitor/dbmonitor-base/src/main/java & 40 & Ensure you override both equals() and hashCode() \\
\hline 6 /home/vinicius/workspace/dbmonitor/dbmonitor-base/src/main/java & 33 & Ensure you override both equals() and hashCode() \\
\hline 7 /home/vinicius/workspace/dbmonitor/dbmonitor-base/src/main/java & 56 & Avoid empty catch blocks \\
\hline 8 /home/vinicius/workspace/dbmonitor/dbmonitor-base/src/main/java & 119 & Avoid empty catch blocks \\
\hline 9 /home/vinicius/workspace/dbmonitor/dbmonitor-base/src/main/java & 98 & Avoid empty catch blocks \\
\hline 10 /home/vinicius/workspace/dbmonitor/dbmonitor-base/src/main/java & 26 & Avoid empty catch blocks \\
\hline 11 /home/vinicius/workspace/dbmonitor/dbmonitor-base/src/test/java & 107 & Avoid empty catch blocks \\
\hline $\begin{array}{l}12 \text { /home/vinicius/workspace/dbmonitor/dbmonitor-base/src/test/java, } \\
\text { /flow/ComparisonFlowManagerTest.java }\end{array}$ & 73 & Avoid empty catch blocks \\
\hline $\begin{array}{l}13 \text { /home/vinicius/workspace/dbmonitor/dbmonitor-base/src/test/java/ } \\
\text { /flow/ComparisonFlowManagerTest.java }\end{array}$ & 93 & Avoid empty catch blocks \\
\hline 14 /home/vinicius/workspace/dbmonitor/dbmonitor-base/src/test/java, & 51 & Useless parentheses. \\
\hline 15 /home/vinicius/workspace/dbmonitor/dbmonitor-base/src/test/java, & 54 & Useless parentheses. \\
\hline 16 /home/vinicius/workspace/dbmonitor/dbmonitor-base/src/test/java & 39 & Do not hard code the IP address \\
\hline 17 /home/vinicius/workspace/dbmonitor/dbmonitor-base/src/test/java, & 49 & Do not hard code the IP address \\
\hline 18 /home/vinicius/workspace/dbmonitor/dbmonitor-base/src/test/java, & 61 & Do not hard code the IP address \\
\hline $\begin{array}{l}19 \text { /home/vinicius/workspace/dbmonitor/dbmonitor-base/src/test/java, } \\
\text { /dao/ComparisonPhaseInstanceDAOTest.java }\end{array}$ & 50 & Useless parentheses. \\
\hline 20 /home/vinicius/workspace/dbmonitor/dbmonitor-base/src/test/java/ & 27 & Avoid empty catch blocks \\
\hline 21 /home/vinicius/workspace/dbmonitor/dbmonitor-base/src/test/java/ & 106 & Avoid empty catch blocks \\
\hline 22 /home/vinicius/workspace/dbmonitor/dbmonitor-base/src/test/java/ & 114 & Avoid empty catch blocks \\
\hline $\begin{array}{l}\text { /home/vinicius/workspace/dbmonitor/dbmonitor-comparison-posts } \\
\text { /HeuristicComparisonCriteria.java }\end{array}$ & 138 & Useless parentheses. \\
\hline 24 /home/vinicius/workspace/dbmonitor/dbmonitor-comparison-posts & 104 & Useless parentheses. \\
\hline
\end{tabular}

Source: [104]

E. Learning/Evolution: Anticipating and monitoring technical debt items was achieved by using the SonarQube tools, the project management tool, and the tool developed by the team itself. Looking at the data that appeared on the TV screen, Team A members could monitor critical points of the technical debt items and could take appropriate action, such as asking staff to pay them down. The data also provided guidance and training on the key issues they faced.

6.1.3.1.3 Third Cycle The objective of this cycle was to begin the technical debt management and payment, which were monitored during previous cycles.

A. Diagnostics: Team A was already able to identify and monitor some technical debt items. In this cycle, they were worried about systematizing the management and payment of the technical debt items. Some Team A members pointed out the need to worry about the quality of the Jmine code, thus raising the need to apply the techniques that were already used to monitor the quality to identifying technical debt items. Another concern raised was how to motivate other company teams to keep track of their technical debt items as a way to improve the quality of the code.

The SonarQube platform has a graphics panel with various code health information, where the quality of the code is observed under the analysis of numerous quality metrics. The problem is that considering all these metrics would be unfeasible and would probably demotivate the team members. Additionally, some metrics were not important to the team, which explains the need to select only certain metrics to be considered at first.

B. Action Plan: The Team A analyzed the context and sought to identify factors that should be monitored, such as recurring errors, for instance. Then, they selected which metrics would apply to such problems and they deliberated on these selections. And they reflected on how they could resolve the technical debt items identified by these metrics. 
In addition, the team planned to make three short presentations (lightning talks) on the three floors of the company. The objective of the presentations was to report their experience in identifying, monitoring and controlling technical debt, as well as dealing with technical debt and improving the code quality.

C. Plan Application: Debts items already registered were analyzed seeking recurring problems and the following measures to remedy them were taken: pair programming, code review and test-driven development (TDD). Other techniques were also implemented, such as considering paying some technical debt items in the team development process.

The code quality metrics selected to be monitored were unit test coverage (Figure 6.3) and technical debt items incurred per day (Figure 6.4).

Figure 6.3: Coverage of Unit Tests

$\begin{array}{ll}\text { Unit Tests Coverage } & \text { Unit test success } \\ \mathbf{4 6 . 2} \% & \mathbf{1 0 0 . 0 \%} \\ \mathbf{4 7 . 8 \%} \text { line coverage } & 0 \text { failures } \\ \mathbf{4 0 . 8 \%} \text { branch coverage } & 0 \text { errors } \\ & 3,622 \text { tests } \\ & 12: 22 \mathrm{~min}\end{array}$

Source: [104]

Figure 6.4: Technical Debt Items Incurred Per Day

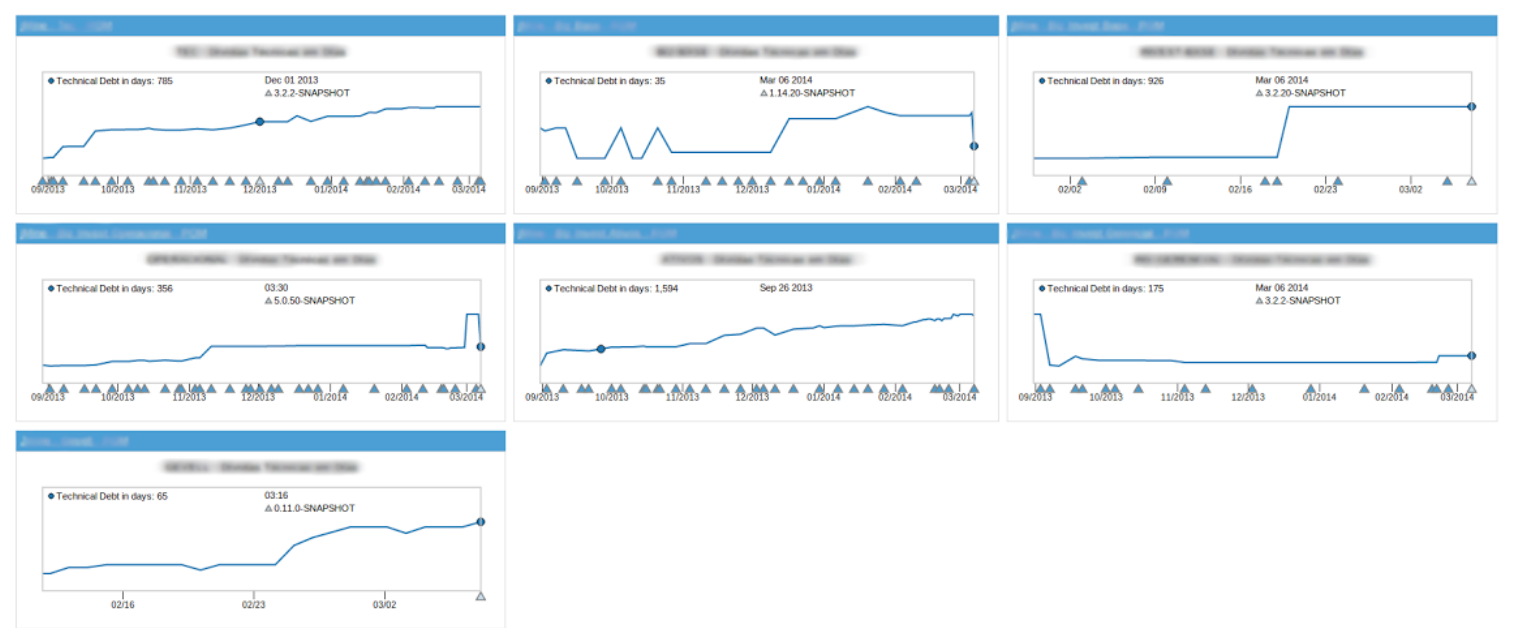

Source: [104]

After having begun to use such metrics, Team A felt that it had significantly improved the quality of the submitted pull requests. As the lightning talks were held, the team got a positive feedback from the other company's teams. They received suggestions to identify, create and document their own standards for dealing with technical debt within the company context.

D. Evaluation: Many teams in the company have abandoned the software development process and good programming practices claiming they were focused on delivering the product on time. Through technical debt analysis, it was possible to observe that these actions could be responsible for the existence of many technical debt items; ergo the tendency was this debt be kept forever in the code due to lack of recognition or recording.

E. Learning/Evolution: By analyzing pull requests automatically, the team was able to received a quick feedback on the health of the code and to persuade other teams to pay attention to the code quality. It also allowed Team A to identify technical and process deficiencies, such as inadequate code review and lack of knowledge of writing good unit tests. 


\subsubsection{Team B}

In this section we will describe how the three cycles of action research were conducted in Team B. Team B was that team responsible for some of the company's products. They commonly worked under a tight schedule and a high delivery rate (product versions).

6.1.3.2.1 First Cycle The purpose of the first cycle was to begin to identify technical debt items.

A. Diagnosis: In the first discussion on technical debt items, this team diagnosed that the produced code did not have the desired quality, lacked automated tests, and that time pressured led to many technical debt items. One member of the team said, "I remember when we were out of time and we were discussing what we should implement, and people believed that making any solution to fix the code would be faster than a definitive solution. Knew the importance of doing something better..."

B. Action Plan: In this team, two members always attended the meetings with Team A and so they knew the concept of technical debt very well. They were responsible for disseminating this concept with the other members of Team B. They also defined a standard pattern for identifying the technical debt items and the type of debt that would be identified.

C. Plan Application: The concept of technical debt began to be discussed individually among Team B members. It was also to focus on identifying a single type of technical debt items since they faced tight deadlines. Only the test debts were considered, given that they were a critical type of technical debt item for this project. This strategy helped the team to assimilate the concept of technical debt.

They started to put the technical debt items in the Scrum board, on which sticky notes identifying the debt items were pasted. The notes had the name of the item written on them and they were separated into "paid" and "outstanding". An example of a board can be observed in Figure 6.5 .

The team also adopted the same strategy in the project management tool including the following information: criticality, estimated time to implement it in hours, location in the source code, and the related task that generated the technical debt. There was a reminder on the board of the fields that should be filled at the moment of technical debt recording, as shown in Figure 6.6.

D. Evaluation: In some aspects, the team was benefited from mapping and taking care of technical debt items: there was an improvement in code quality and identification of some points of attention in the code.

E. Learning/Evolution: Through the list of technical debt items on the board it was possible to obtain visibility and preliminary evaluation of the code health. For this team, one of the most important benefits was that team members were aware of the quality of the code each time they would incur a technical debt item.

6.1.3.2.2 Second Cycle In this cycle, Team B continued to improve their process for identifying technical debt and began to use some technical debt monitoring metrics.

A. Diagnostics: Even with the chart and registration of technical debts, Team B believed that it was necessary to gather more information about each item to facilitate their payments.

B. Action Plan: Team B planned ways to show the real importance of identifying technical debt. They added items information, such as context and initial technical debt item analysis. Because of the difficulty in taking the time to pay the technical debt items, a team member was assigned 
Figure 6.5: Board with the Technical Debt Items of Team B in Company B

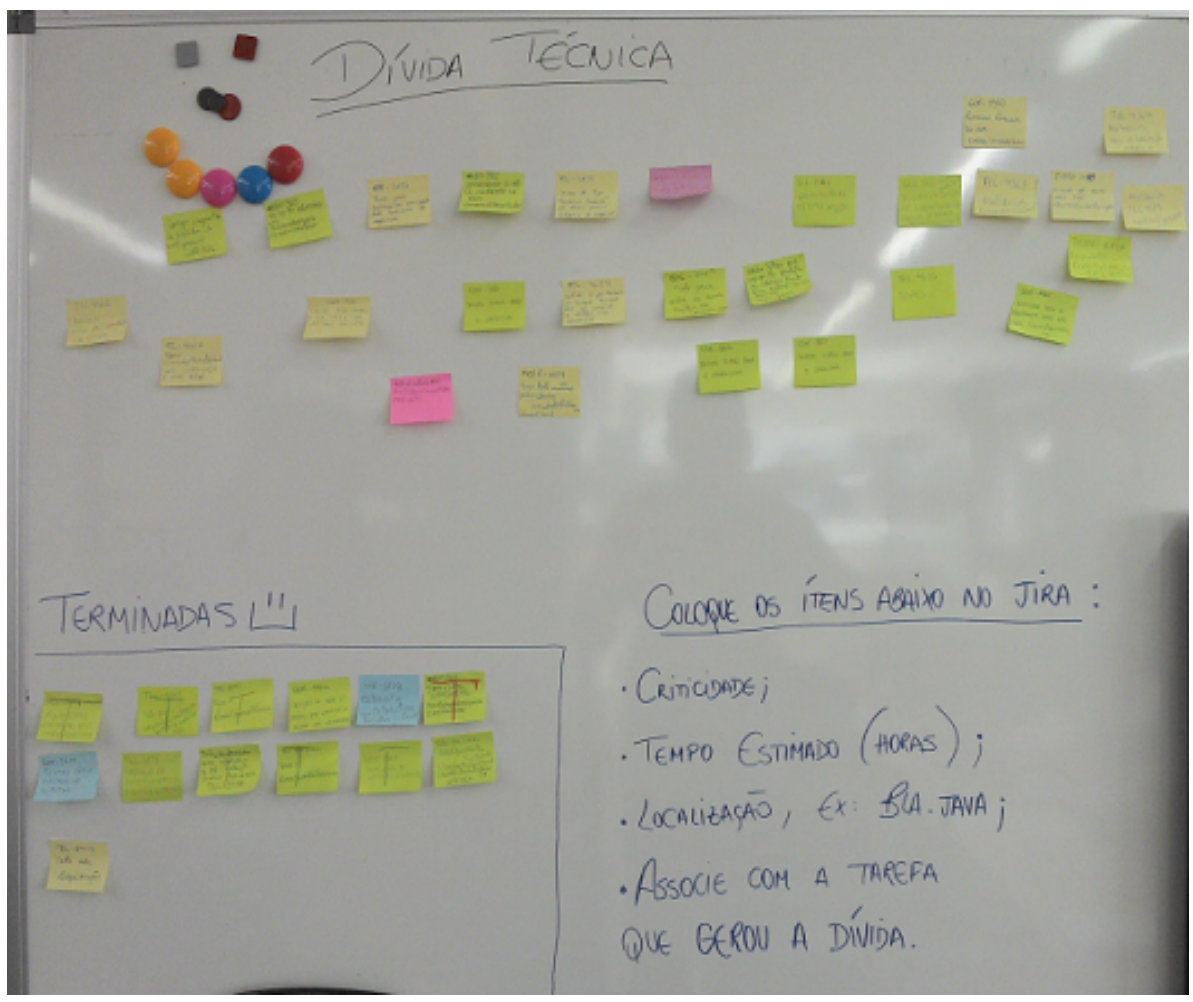

Source: [104]

Figure 6.6: Fields that should be Completed in the Technical Debt Form.

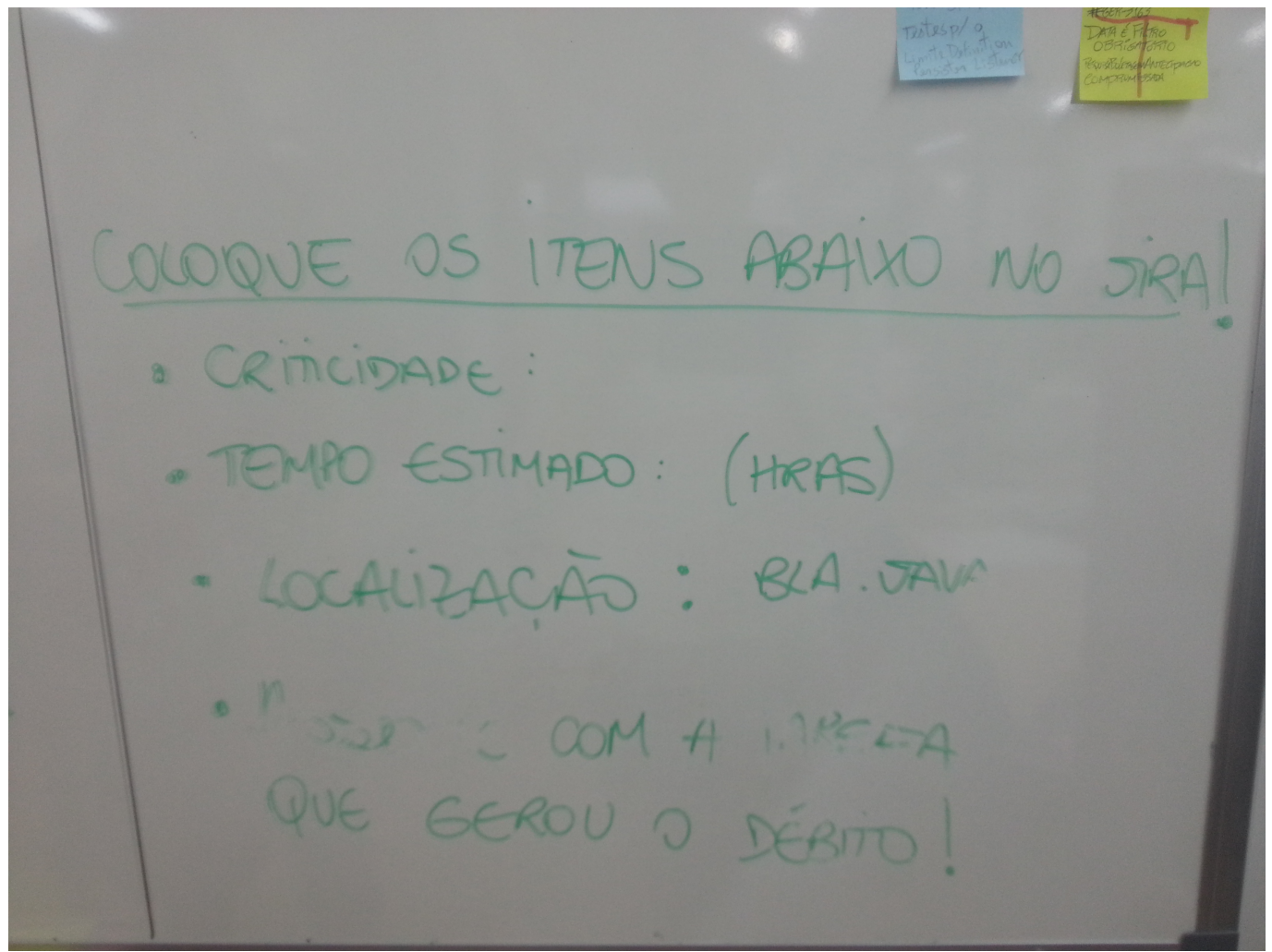

Source: [104]

to pay them. Although the team was aware this was not the best solution, they knew it could temporarily help them to start showing the value of monitoring technical debt for the team, 
managers and leaders.

C. Plan Application: At their planning meetings, Team B started discussing the time needed to pay the technical debt items. A team member was responsible for paying them.

D. Evaluation: In this cycle, Team B started to visualize some weaknesses of their application, which helped improve their awareness about the importance of the technical debt issue.

E. Learning/Evolution: Through the use of the chart and the form in the management tool, Team B improved their perception of the technical debt list of their project. This also helped them to revisit the technical debt items once they were paid. Assigning a team member to paying off technical debt items addressed the problem of how to integrate technical debt payment into their development process. However, the team was aware that this was a temporary solution, since it was not motivating for the developer to continue paying the technical debt items that were created by the other team members. This action could also generate a certain accommodation of the team, since there would always be someone to correct the technical debt items inserted by them.

6.1.3.2.3 Third Cycle In this cycle, the team's aim was to evolve the monitoring and payment methods of technical debt items.

A. Diagnosis: Team B decided to stop and analyze the technical debt items already recorded. However, they realized that over time the team members started to record fewer technical debt items and it became clear that they needed to motivate the teams to continue recording them. It was also necessary to start automated code analysis.

B. Action Plan: Team B planned to start using SonarQube to analyze the project code and select the first quality metrics to be considered. In addition, they planned a way to encourage all the team members to continue recording and star paying the technical debt items. The team intended to adopt pair programming techniques to improve the quality of the code and to encourage members to pay some technical debt items. Furthermore, the team planned to present their first results in a quick conversation on the other floors of the company along with Team A and the Research and Innovation team.

C. Plan Application: Some technical debt items were paid after pair programming was adopted. The team began analyzing reports from SonarQube and the management tool to find recurring problems.

D. Evaluation: In this cycle, it became clear to Team B members that incurred technical debt was not necessarily bad, although it was a big challenge not to forget to pay it off.

E. Learning/Evolution: Team B in this cycle was focused on making clear to all of the team members the importance of raising awareness about technical debt items. Some technical debt items were paid with the effort of a single member of the team, even though the team was always aware that this was not the best solution. In contrast, by monitoring the technical debt items in a collaborative way through tools and periodic pair programming, they realized that the team's behavior had improved. Team members, managers and leaders have come to be more supportive of the idea of considering technical debt in the upcoming iterations. 


\subsubsection{Results}

In this study, the action research method was applied and three cycles were performed in two of the company's teams.

The teams used the Jira tool and a Scrum board to identify technical debt items. They also used the SonarQube tool to monitor the debts. They separated a specific part of the Scrum board to put the technical debt items, as we can see in Figure 6.7

Figure 6.7: Technical Debt Items on the Scrum Board.

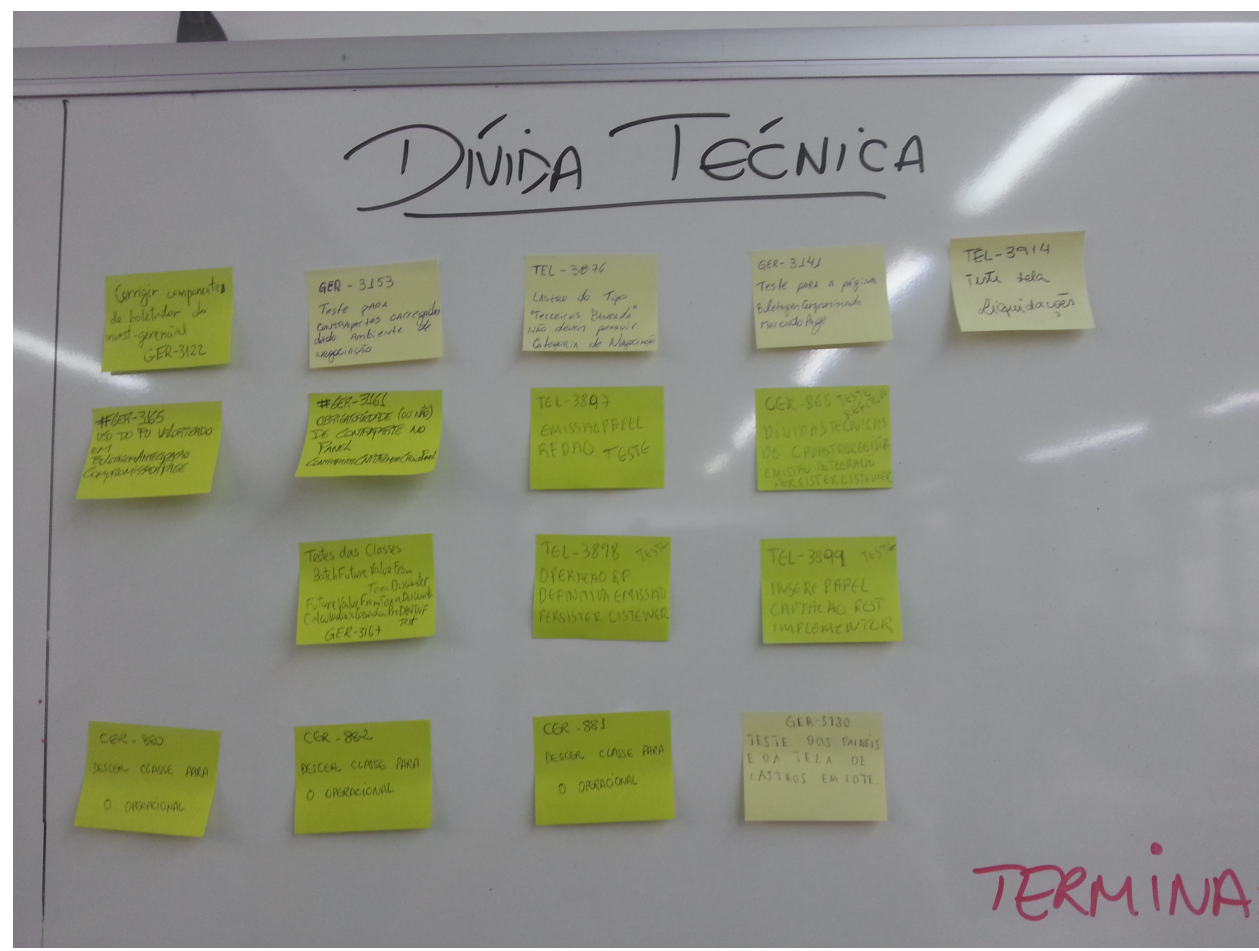

Source: [104.

Each sticky note had a technical debt Jira ID and a brief description of it.

Furthermore, in the Jira form the team member could fill out the technical debt criticality, estimated principal, location, priority and connect the technical debt item with the task that originated it. In Figure 6.8 we can see an example of the technical debt item in the Jira tool.

Figure 6.8: Technical Debt Item in the JIRA Tool.

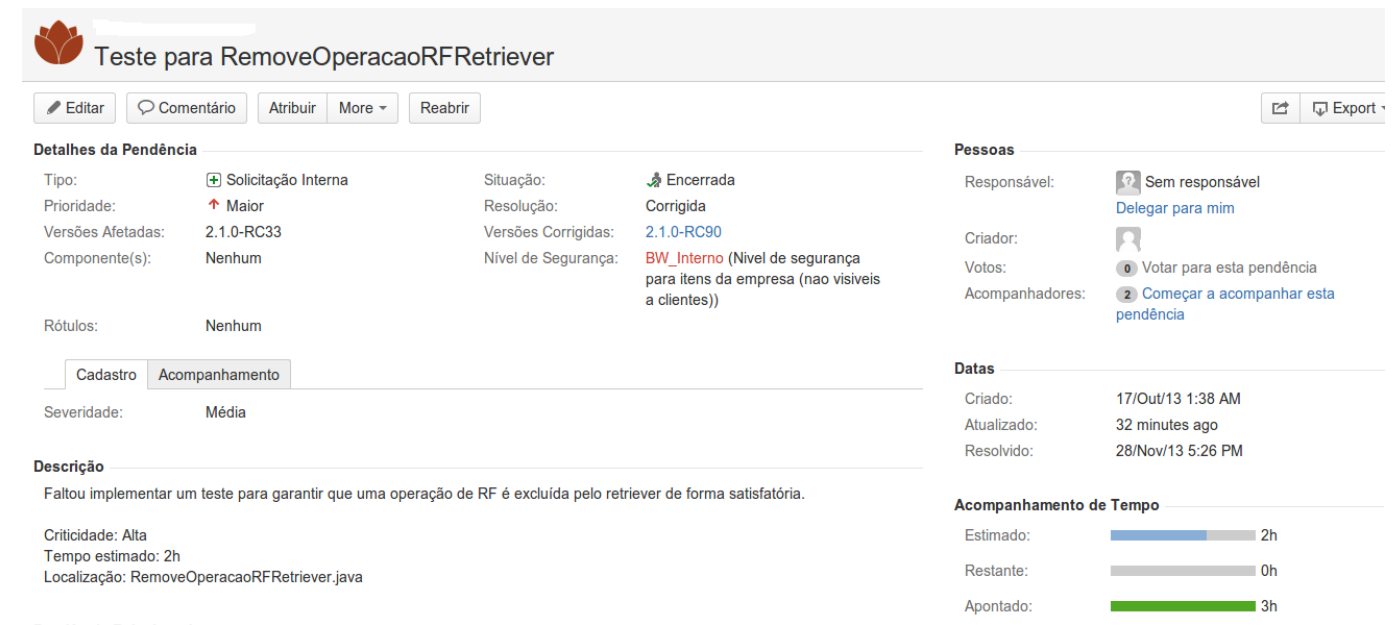

Source: [104]. 
In this case, the technical debt item was about a lack of test, the criticality was high], the principal estimated was 2 hours and the priority was major. They also related it with the software version where they identified the technical debt item and the software version in which they paid the technical debt item. Once this technical debt item was paid, they filled in the information about how much time they spent to pay the item, which was 3 hours. They also put the information about who paid the technical debt item, although due to confidentiality we have omitted that information. Finally, we can see the date the item was identified and the date the technical debt item was paid.

The teams also used the reports provided by the Jira and SonarQube tools to monitor the technical debt items. The reports included a list of technical debt items incurred and paid, Figure 6.9.

Figure 6.9: Technical Debt Items Incurred and Paid.

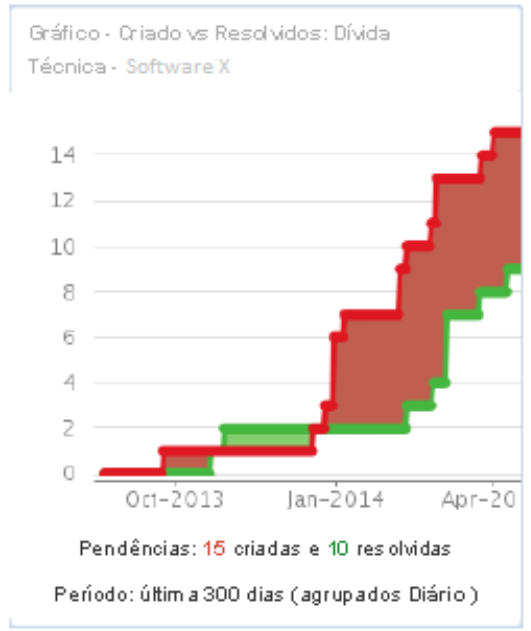

Source: [104].

The red line is the total number of all technical debt items, and the green line represents the technical debt items that were paid. In this case, they incurred 15 technical debt items and paid 10 items.

The team also used a graphic on the TV screen, as we can see in Figure 6.10.

Figure 6.10: Amount of Technical Debt Items Inserted in the Software Versus Code per Team.

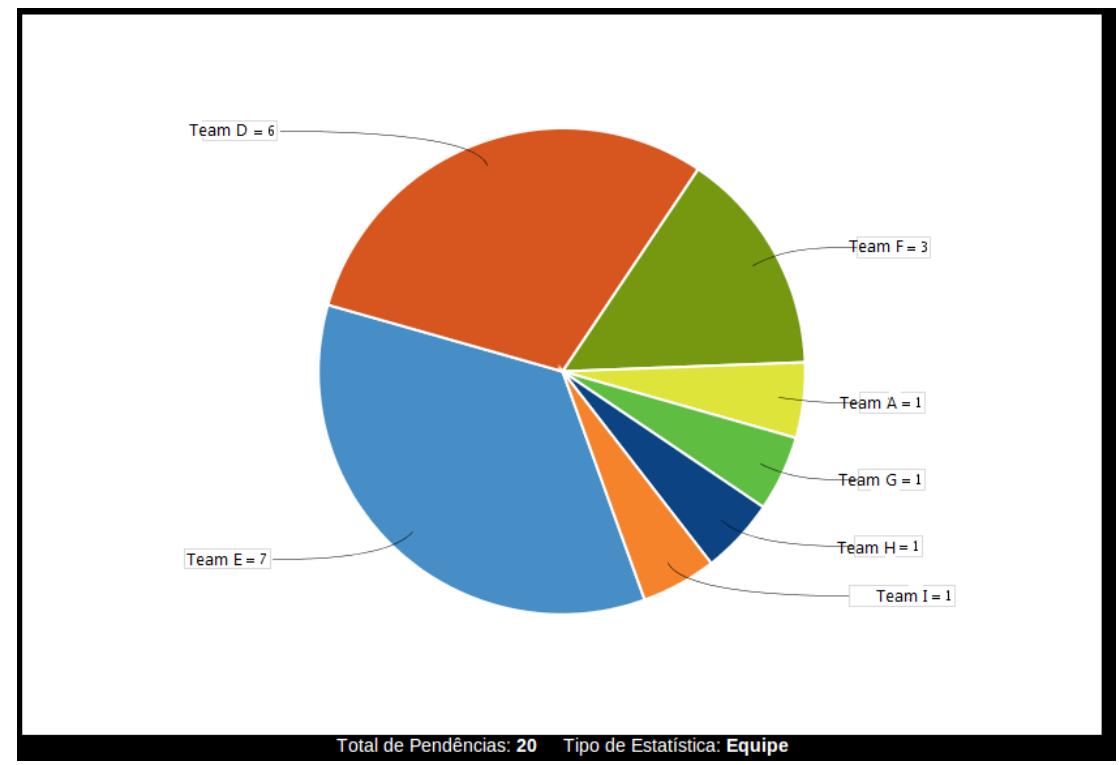

Source: [104]. 
This graph shows the number of technical debt items that were inserted by the team in he code of Jmine.

Regardless of the form of monitoring, a meeting would be held to discuss whether a technical debt item should be paid or not. If it was decided not to pay the technical debt item, it must continue to be monitored. If they chose to pay it, it must be added to the project's backlog and could be paid during sprint development, or they could plan to pay it on a day reserved to improve the quality of the project, such as a refactoring day.

As a result, we created a conceptual model for technical debt management that we explain in the next chapter.

\subsection{Chapter Summary}

This chapter presented the studies we did in Company B, where we conducted a study in two different agile teams. First, we discussed the three action research cycles we performed in two agile teams. In each cycle, we showed the challenges the team faced and the actions they took to improve how they dealt with technical debt items. These teams used a Scrum board and the Jira tool to registered the debts. In addition, they used the tools SonarQube, PMD and they created a plugin to monitor the debts. TV screen and charts were used to make the items visible. Based on these studies and on the academic studies we create a conceptual model for technical debt management aimed to help teams to consider technical debt. 


\section{Chapter 7}

\section{Conceptual Model for Technical Debt Management}

This chapter compares the last version of the conceptual model for technical debt management with the models we found in the literature. Its important to emphasize that we only compare with those models that directly consider all the activities of technical debt management or it were considered a conceptual model. We discuss each analysis in the next sections.

\subsection{The Model}

The conceptual model for technical debt management, which we can see in Figure 7.1. includes the findings of the studies we did in Companies A and B and in the Academic Studies.

The central steps of the technical debt management process are based on the model of Seaman et al. [87. This is the third version of the model, and it was created after we had finished the studies. In this version, we have an abstract and yet clear vision of the main areas the teams must consider in order to manage technical debt. The studies showed that instead of just following the main activities to manage technical debt, the root causes of the debts could be originated by issues that are not directly connected to the code or the project, for example, a business decision. Thereby, we cannot ignore them and to mitigate the recurrence of this type of debt the teams have to take actions to solve or mitigate the root cause. They can solve the problem and focus on prioritizing other essential aspects of the software, such as the software evolution or other types of debts. While they have to consider the activities for technical debt management in order to adequately manage the technical debt items, it is crucial to investigate the causes and whether the context influenced the incurrence of such debt into the project.

With that in mind, the model was improved and its fourth version was developed. This version is a detailed version of the previous conceptual model where in addition to the activities for technical debt management were added the techniques and artifacts to the whole process - from understanding the metaphor to making the decision of paying the technical debt items, as we can see in Figure 8.1 .

It can be observed that in order to manage technical debt in the cases studied, we identified four main areas that are important to consider and monitor when dealing with technical debt items: the technical debt impact, the technical debt causes, the context where the technical debt is incurred, and the steps of the process of technical debt management - identifying, measuring, monitoring, and payment. But also it is necessary to categorize and prioritize the technical debt items. Additionally, the model suggests one way for technical debt management considering important points for decision making. For this purpose, the model was divided into two main parts: in the first part, the focus is on technical debt identification, and in the second part, the core is composed of the activities to manage the identified technical debt items. 
Figure 7.1: Conceptual model for Technical Debt Management.

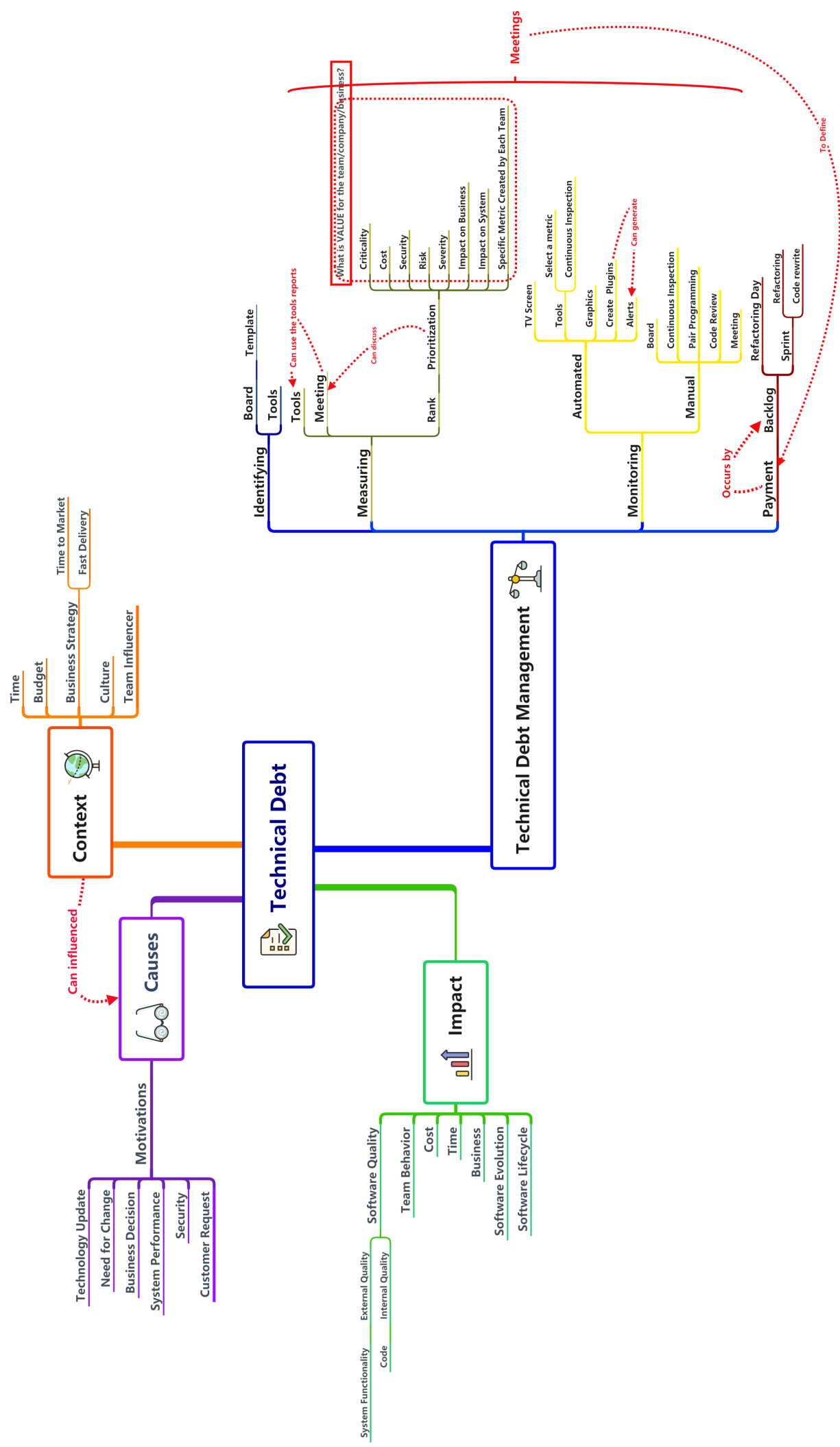

\subsubsection{Technical Debt Identification}

In the first part, as we can observe from Figure 7.3, the model starts with the Technical Debt Metaphor, where the management of the technical debt items depends on the team's understanding 
Figure 7.2: Conceptual Model for Technical Debt Management

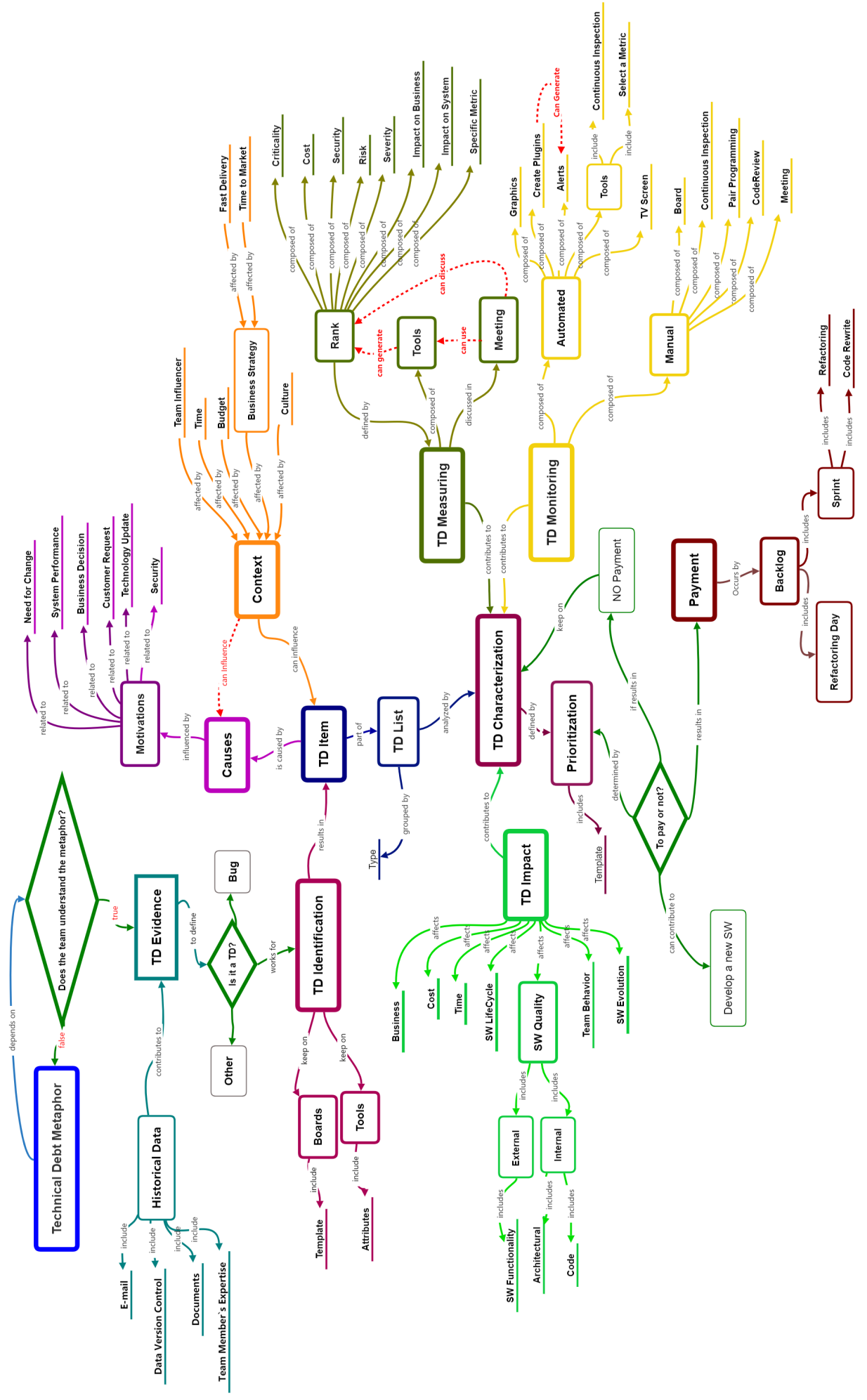

of the technical debt concept. One of the evidence for reaching this understanding is by making an analogy between the technical debt metaphor, and for instance, some examples of code that were implemented and were not good enough. Indeed, they have to understand the technical metaphor in order to define what is the most relevant technical debt for each project.

Once they comprehend the technical debt metaphor and they defined some examples of the technical debt in their project, they can create a template to collect evidence of the technical 
Figure 7.3: Technical Debt Identification

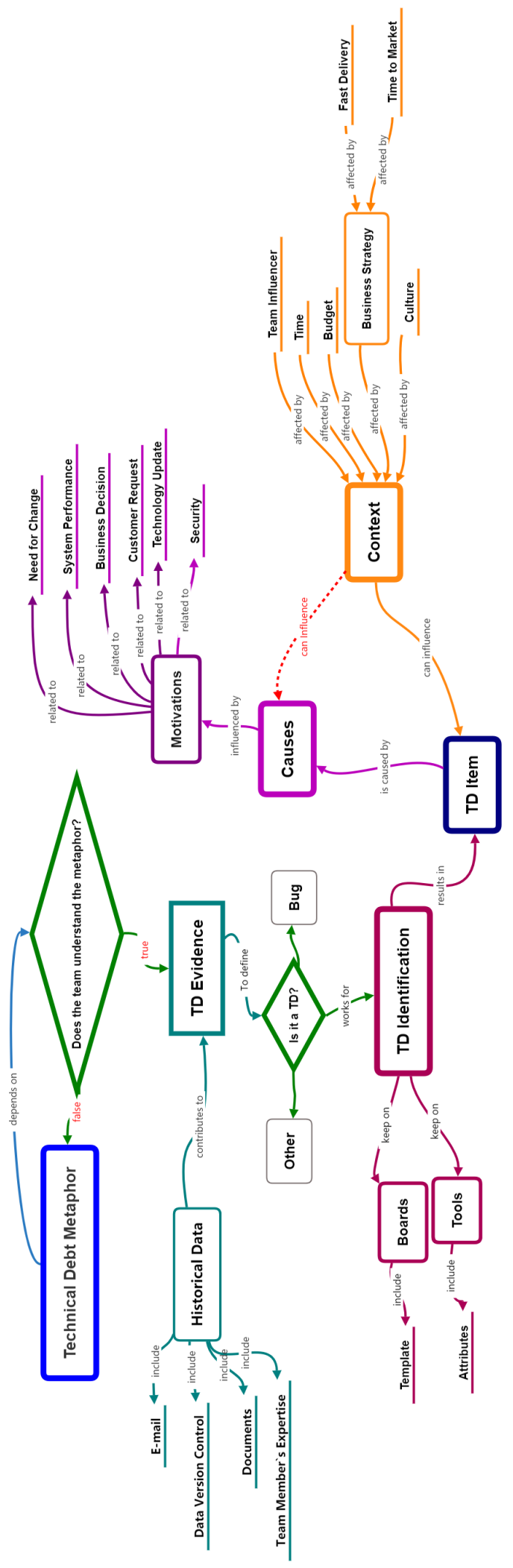

debt items (Technical Debt Evidence). It can be done by code analysis with tools for software quality monitoring and by analyzing the historical data - as the significant changes in the code, emails, documents, team member's expertise, data version control, etc. The tools could help them 
identifying evidence on the existence of technical debt on some parts of the code. However, when using the tools to identify technical debt they would often need a second human analysis to be able to determine accurately whether the item is in fact, evidence of technical debt.

In the studies at Company $\mathrm{A}$, we started to collect evidence of technical debt by talking to the team members about the software's immature code and identifying the past significant changes in the software. We also used the software tool's report to identify when and where it happened specifically..

Once the teams have identified evidence of technical debt, they can classify it as a Technical Debt Item, bug or other. Considering that they have found a technical debt item, the next step is to collect information about the item, such as a brief description, location, ID, type and tasks that are connected with this debt, among others. The teams can put the debt data on a board and/or in a tool. In the studies, we followed the template suggested by Guo studies [42] to collect the information on technical debt. We tested the template and putt all the data collected on a technical debt board. In the in-company studies, we decided to put just a few information of the item on the board, for example, ID and name, because we found that putting all the information not only takes more time, but it also leaves the board with lots of information which makes it difficult for the developers to focus on just how many technical debt items the project has and what exactly are the technical debt items. The remaining data we put in the Jira tool, which was already used in both Companies (A and B) to manage the tasks and bugs. In most of the Academy's teams, we had a specific board on which we could display the technical debt items. In the Companies studies, we put them on the Scrum board jointly with the other tasks.

These incurred technical debt items can be influenced by the context, for instance: time, budget, business strategy (e.g. time to market, fast delivery), the culture of the company, and because of a team influencer - a team member can convince others to adopt a particular technology because he/she considers it the best option. The context can influence the motivations that lead to technical debt incurrence. Many of the technical debt items we analyzed were originated in a business decision or because of the expertise of a team influencer. Some interviewees pointed out that factors such as company culture and time were the real motivations of the project to present that kind of technical debt. These motivations can be technology update, a business decision, a need for change, to improve the system performance, because of some security rules or security deficiencies, and also because of a customer request. The motivations are directly responsible for the causes of the technical debt.

As a result of the data collection on the technical debt item, the teams will have a resulting list of technical debt. This list must be categorized according to themeasure and the impact of the technical debt item. At this point, we consider the beginning of the second part of the conceptual model for technical debt management.

\subsubsection{Technical Debt Management}

In the second part of the conceptual model for technical debt management, as we can see in Figure 7.4. has the focuses on the activities of technical debt management.

After, the teams have the technical debt list they have to decide which technical debt will be their priority. For that, the teams have to do the technical debt Characterization. This characterization includes to Measure the technical debt items, defining metrics and analyzing their Impact. In order to measure technical debt items, we suggest three approaches: the first is still using the software quality tools to measure some types of technical debt items such as test. These tools can estimate the number of technical debt items and some tools even provide a specific value of the technical debt items in the code. The second suggestion is to discuss it during the team planning meetings - whether estimating meeting, prioritization meeting with the customer, or in the sprint planning meeting - where the teams could measured the items along with the planning of other activities. The third approach is to create a technical debt rank with the list of technical debt items that would allow the definition, by project, of which technical debt item is the most crucial to be paid in each sprint. This list of technical debt items can be prioritized by cost, criticality, impact on the system, impact on system's security, impact on business, risk and severity. The teams, can also 
Figure 7.4: Technical Debt Management

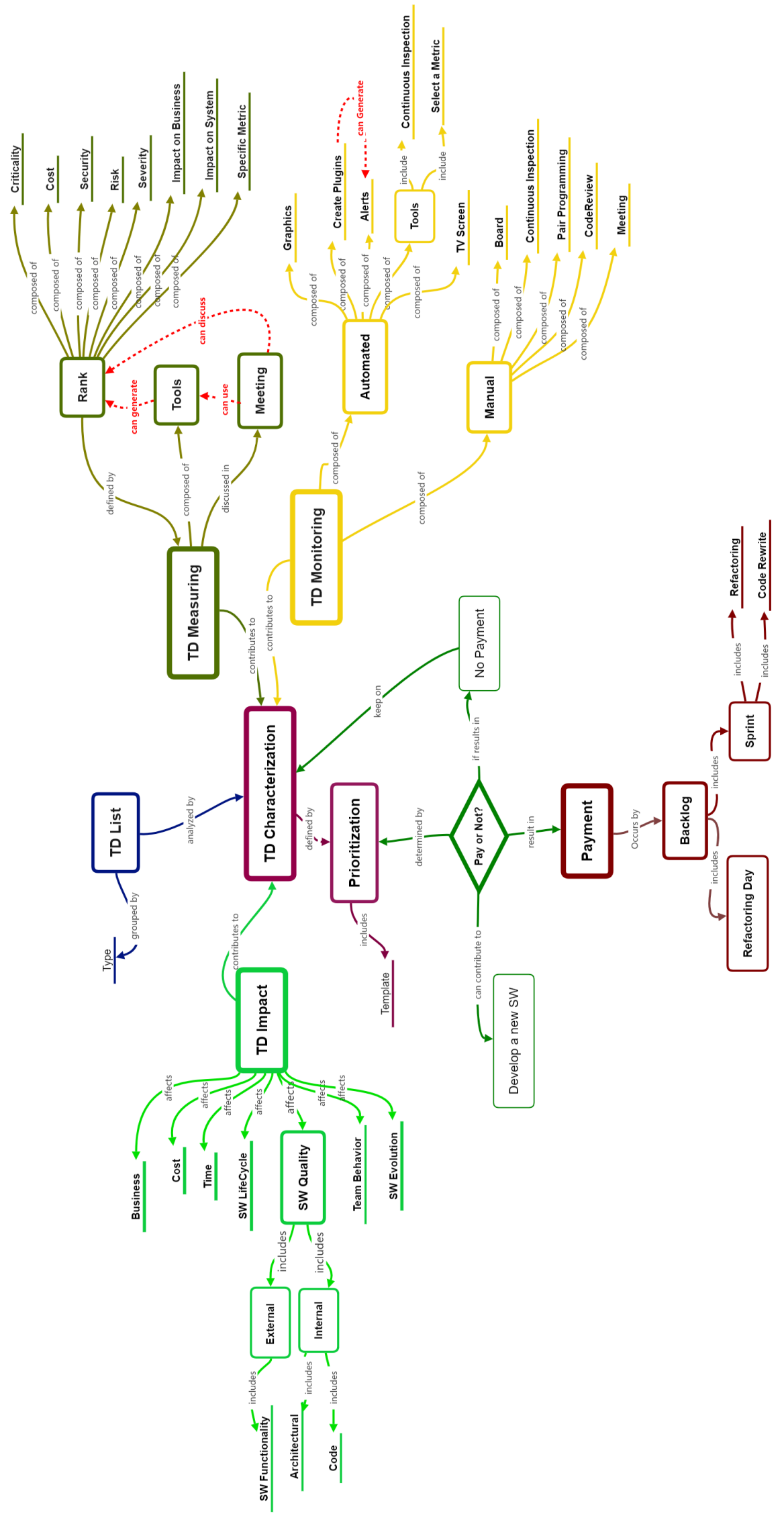


create a specific metric associated with the project, the team, and the customer goals.

Likewise, the teams can use the tools reports to help them organize the technical debt items rank. At this moment, it is essential that the team has defined what is 'Value' for the project, so they can determine what is crucial to prioritize. In the cases studied, too often the rank was created by organizing the technical debt list by type and setting a value for each of them. To generate the rank, they decided which type of technical debt was more critical, so they considered to pay down the technical debt according to a pre-defined list of the most critical types of technical debt items for that project. For instance, test coverage was an important metric for some projects and if some technical debt was about that, they considered paying it off. After the teams define the metrics, they must monitor the technical debt items in order to manage them. The teams can do it by the manual and automated approach. Manual depends on people, and it includes keeping the debts on a board, creating a technical debt list, doing pair programming, etc. In figures 5.18 and 6.5 , we have examples of how teams have structured their boards. In order to put the data about the technical debt item on the board, it is necessary to create a template with the items that should be listed on each technical debt card, as well as how and where these data will be inserted. This approach is simple and clear and it helps to make the team aware of the code health. Additionally, in each sprint planning meeting, the teams can analyze the list of the technical debt items and discuss it. The automated approach includes tools where data could be inserted to monitor the technical debt items. Once they have these data in a tool, they can automate the technical debt monitoring since it is easy to generate graphs and reports on the list of registered technical debt items. It is also possible to develop a plugin to monitor when technical debt is incurred and then set up an alert for each technical debt item found - it could be an email communicating it and/or the update of a real-time chart. Moreover, it is possible to configure it so that whenever a certain action occurs or at certain periods of time, it will monitor the desired events (code, builds).

The teams used a SonarQube tool to monitor the test coverage of the code. One of Company B's teams created a plugin to control the acceptable level of the quality of the merges in the framework of the system. There, TV screens were also used to show the graphs related to the technical debt items incurred by the team. An example can be seen in Figure 6.10. Furthermore, they can monitor the technical debt items using practices such as continuous inspections, pair review and pair programming. In the studies, at the first time we considered the tools report, we did not choose specific metrics to monitor the technical debt items but it resulted in an uncomfortable and unmotivated team due to many reports that never showed a significant improvement in the software quality. As they considered a lot of metrics, the team tried to pay down many technical items, and despite their efforts, the reports always showed that the software quality was poor. So the team had to define a set of metrics, reorganize and review them over time in order to consider only the relevant metrics for that project and monitor the technical debt items that were more relevant to them. The team defined the rules, metrics and approaches to categorize the technical debt list, thereby collecting the proper information that enabled them to define which technical debt they would prioritize to pay. As a result the list of technical debt items is recorded and its history can be analyzed. Since it is possible to make a historical analysis of the technical debt items, it also becomes possible to identify patterns that always result in technical debt items, such as teams knowledge deficiency in a certain technology. This way, the team can even define training on such technology to mitigate the occurrence of that problem.

Now that the team has collected information on the debts, they can define the technical debt Prioritization. According to this prioritization, they can decide whether to pay the debt. If they do not pay it, the technical debt item has to be categorized as unpaid technical debt so they can continue monitoring and analyzing it. Although some technical items need to be paid, not all of them do. So, if they did decide to Pay any, the team has to analyze the technical debt list and choose the technical debt item that it is crucial to payoff. They can consider the technical debt on the project backlog and either pay it or choose a specific day to do it, such as a refactoring day, or even set it as one more task of the sprint to be implemented by refactoring and sometimes by code rewriting. 
We used the model to make two examples of its application for technical debt management, following the data of the studies. In one the examples, the data are coming of the XP Lab 2014, and in the second example, the data are coming of the Company A data. We can see the examples in Figure 7.5 and Figure 7.6 .

In Figure 7.5, the team had a talk and discussed the technical debt management. Then, they chose to consider and monitor test coverage as technical debt. For which they used the SonarQube tool. When we asked whether they used a tool to monitor it, they answered: " Yes[..] SonarQube.". Although this tool provides a report of the many static code metrics, they considered only the test coverage.

The team had the technical debt item called test coverage listed in Scrum board. The level of test coverage was low because all team members decided not to prioritize test implementation due to the implementation of the new features(features requested by the customer). They justified this low level, because of the lack of time to do that. For the rank was considered the technical debt of test coverage type. They believed that its impacts the software evolution, so in their understanding, it has high criticality. A team member said: "Yes. Because the project will probably be continued by another team.". In each sprint planning meeting, they decided whether they would spend any time to implement tests. They considered the tools report to measure and monitor the technical debt items. A team member said: "We did not worry about it until we saw the sonar." other team member said, "Yes. Because they indicate elements that should receive more attention.". The team members also monitored the tests of the software during the codification following the pair programming practice. They also said that choosing to consider technical debt items would impact the software evolution. Another team member said: "Yes. It is an important factor to the survival of the project.". Additionally, they emphasize that it motivates them to increase the test coverage, "...increase the number of tests where necessary". Finally, they said that considering technical debt is a stimulating factor to solve the failures: "Yes, it is a stimulating factor for the team to resolve failures." Sometimes they would defined a task in the sprint to refactor the code and improve the test coverage.

In the second example, we followed the data collected in Company A, as we can see in Figure 7.6.

In these studies, the researchers talked about technical debt to some technical leaders in Company A. After the team members had understood the technical debt concept, they decided to start analyzing it on their projects. In order to identify whether the project had any technical debt items, the researchers and team members, decided to start analyzing the big changes occurred in the code. The team members suggested a software that, in their opinion, presented technical debt evidence. Four big changes were selected to be analyzed by historical data and interviews of the team members. They inspected documents and the registration of the version control, and an interview was conducted with the team members that worked on the project at the moment of that big changes occurred. As a result, we were able to identify the technical debt items incurred. Afterwards, we start to collect evidence that could lead us to the root cause of the technical debt incurrence. As a result, we found that the root causes were originated mainly because of the company culture to impose business decisions on projects and often because of a team influencer. We also analyzed the impact of these debts and the results showed significant impact on the project such as many men/hours invested in adapting the software due to business decisions, an outdated product compared to competitors, need to develop a new solution because it was impossible to maintain it due to problems of code performance and complexity, and the team's high level of stress. One project was delayed by 30 days and another, by 120 . Due to these results, the teams started to define and test strategies to start considering technical debt in their projects. They set a template to put the debts in a Scrum board, chose the attributes to put in the Jira tool - ID, name, type, and description, among others - defined tools to monitor and help them to identify the impacts, such as the Jira tool. The decision of paying or not the debts was made in the sprint planning meetings when the team and the Product Owner of the project reached a conclusion of which technical debt could be paid on that sprint and which debts they would keep on the list to continuously monitor, and made a decision based on the impact on the system. In some cases, they decided to implement a new 
Figure 7.5: Conceptual Model for Technical Debt Management according to the Academic Studies

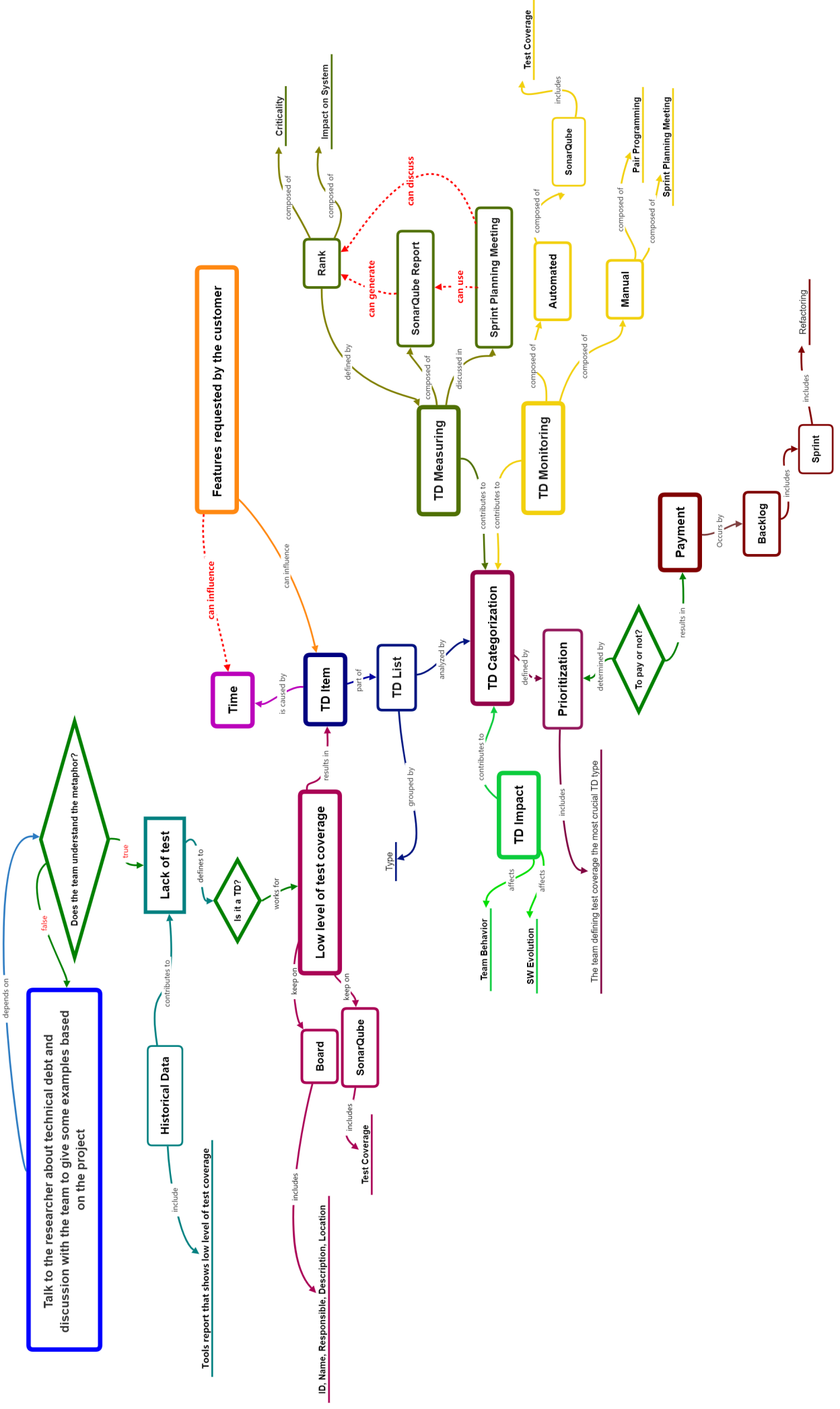

solution with new technology. The debts they choose to pay were inserted in the sprint backlog and paid by code refactoring or rewrite.

This model represents the understanding generated from the data analysis of all the studies we conducted in this research. It can help teams to thoroughly organize from how to identify technical 
Figure 7.6: Conceptual Model for Technical Debt Management according to the Company A Studies

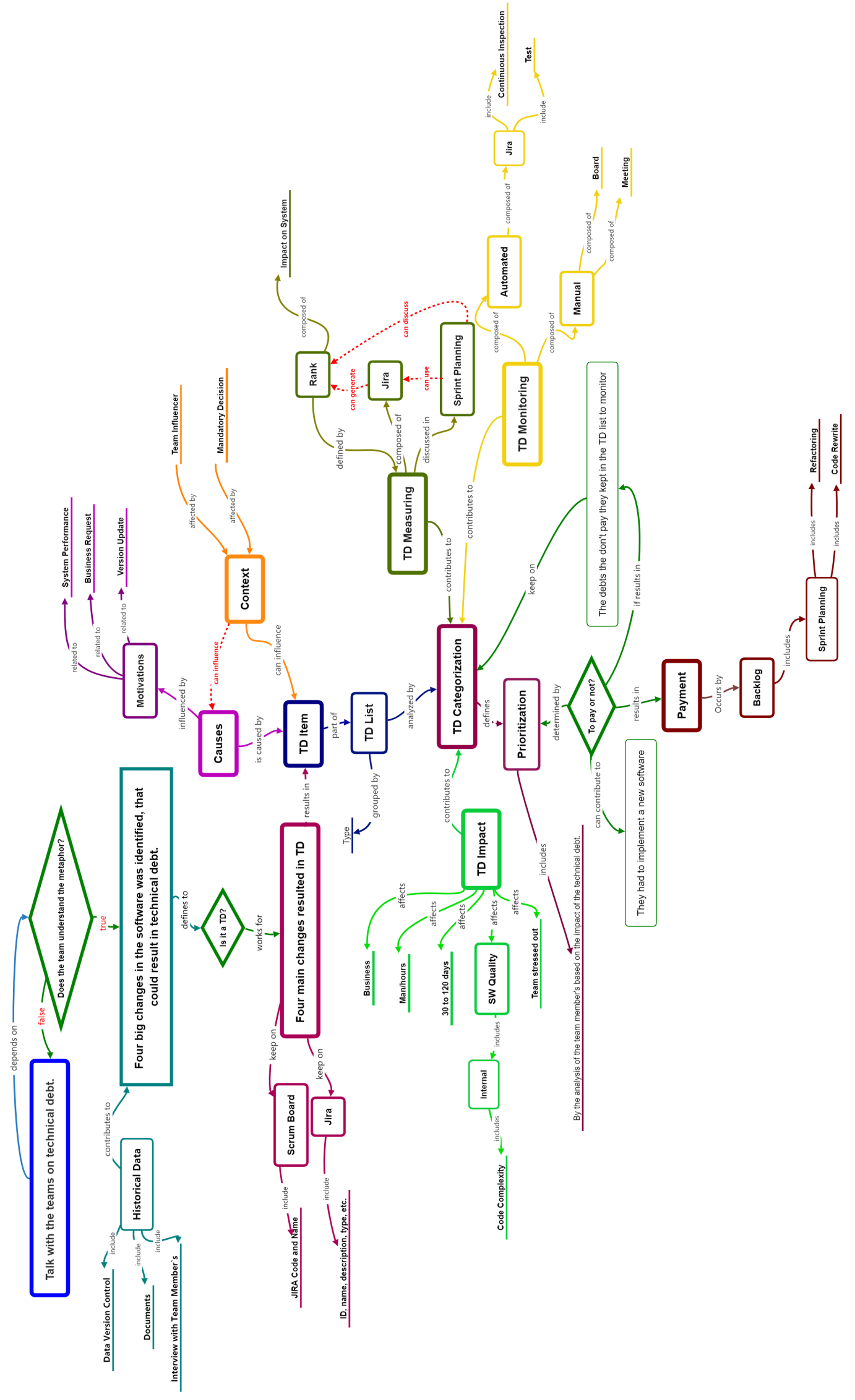


debt items to technical debt management. Each part of the model can be associated with the tools, methods, techniques, and processes already used by the teams. This model could improve the visibility of the software quality as well as it can help companies to identify weaknesses in the process of decision-making, showing improvement points in the team's technical skills, technology and tools.

We analyzed the literature and chose five main pieces of research that are directly related to a conceptual model for technical debt management. One of them is the Seaman et al. model [88, which we followed to develop this study. Another model is the Harun et al. [43], they created a framework to manage and reduce technical debt items based on cost-benefit analysis (CoBeTDM), focus in the activities: identification \& assessment, monitoring, quantification \& prioritization and repayment. The other is the study of Avgeriou et al. [4] that create a conceptual model to discuss some aspects of the technical debt management. Also we compare with the Huumo et al. [109] study, that investigated eight software development teams to understand how they deal with technical debt. In this study, they discuss each technical debt management activity. Finally we compare with the Besker et al. [9] model that created a conceptual model to manage architectural debt. In the next sections, we are going to compare and discuss these models.

\subsubsection{Comparison with the Models}

\subsubsection{Framework for Technical Debt Management}

As we previously said, the model created by Seaman [87] was the model we follow to start the studies aimed to create a complementary model to technical debt management. Since we are conducting this research many studies arrive about technical debt and some of them proposed a model to technical debt management.

The first model we compared is the model created by Seaman [87, in this model, it is suggested that to manage technical debt, the teams have to identify the debts, make them visible and identify their causes. We can see the model in Figure 7.7.

Figure 7.7: Framework to technical debt management.

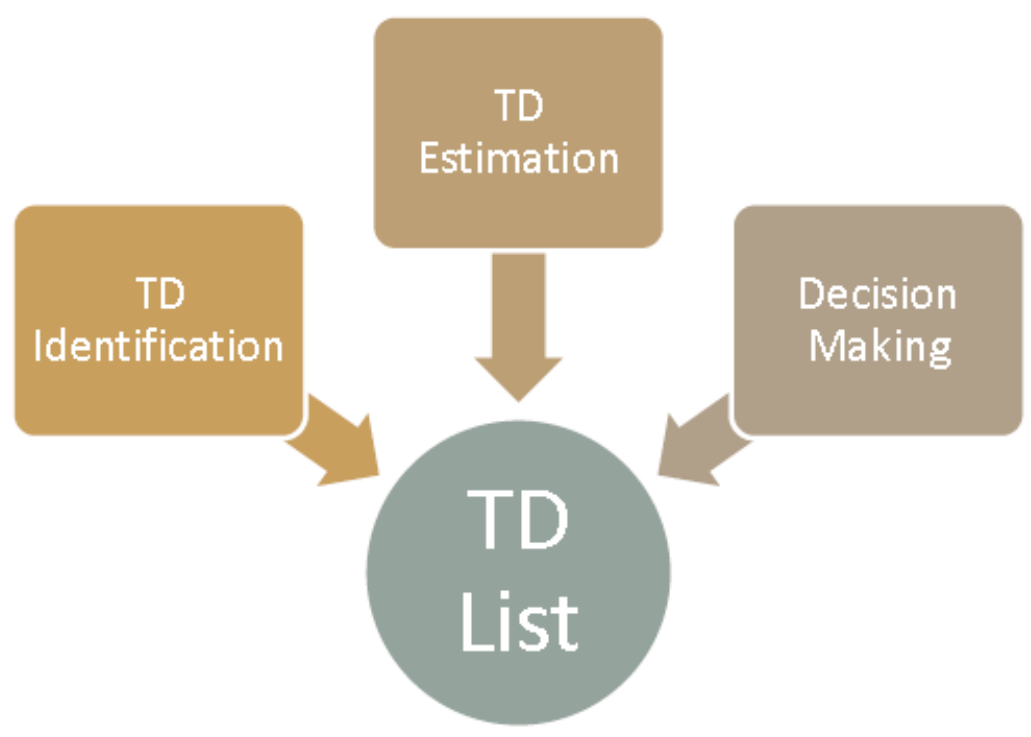

Source: [87]

The model also recommends estimating the effort required to pay the debt then make the decision to pay off it or not, or keep monitoring. In our model, we follow this activities of technical debt management and tested some techniques and approaches to doing that suggesting how to apply each of theses activities. In addition, we recommend complementary activities to technical 
debt management as defining the evidence, defining the characterization and to prioritize, etc.

\subsubsection{Framework to Manage and Reduce Technical Debt}

Harun et al. [43] created a framework to manage and reduce technical debt items based on the cost-benefit analysis (CoBeTDM), focus in the activities: the identification \& assessment, monitoring, quantification \& prioritization, and repayment, as we can see in Figure 7.8

Figure 7.8: Framework to Manage and Reduce Technical Debt

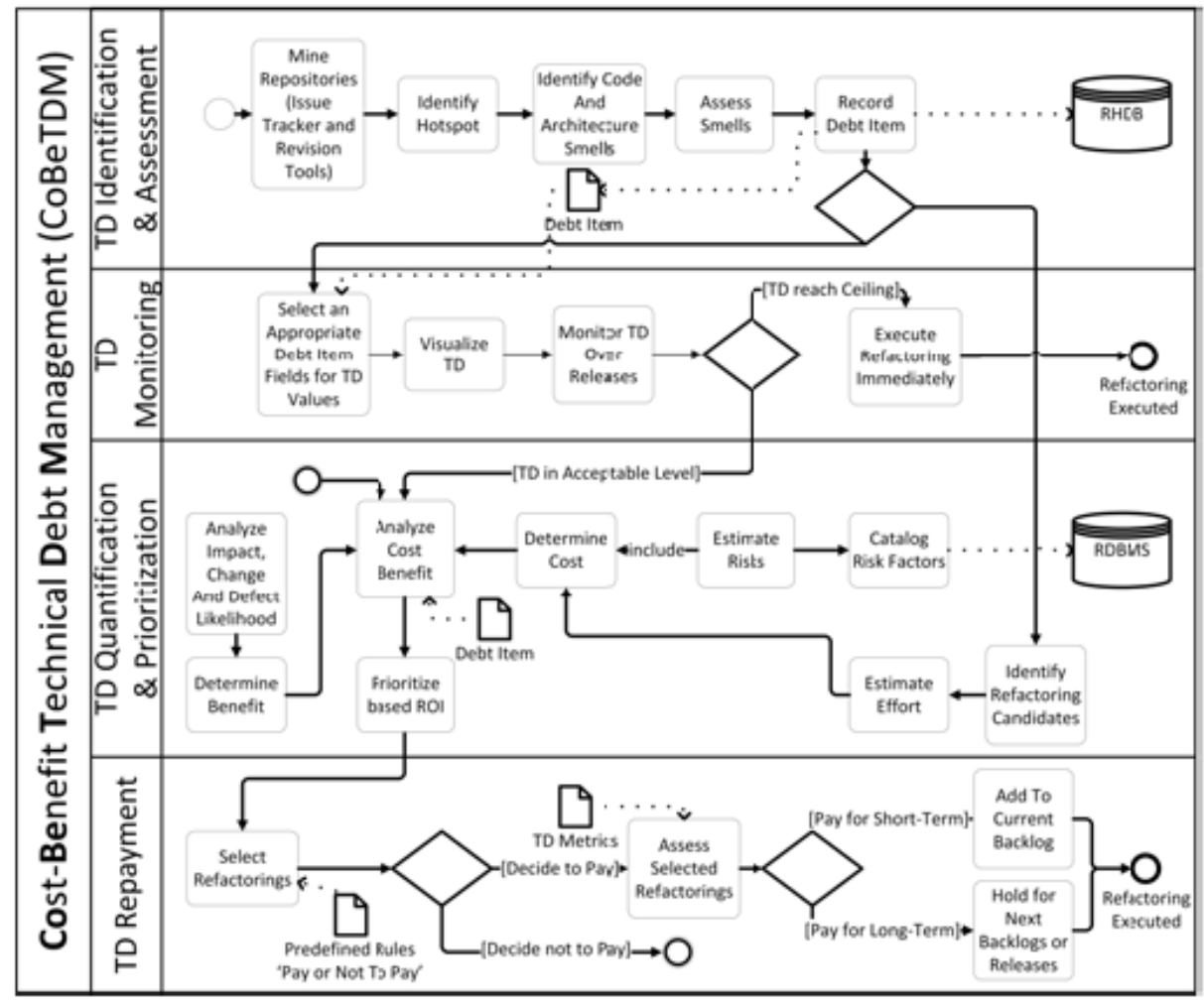

Source: [43]

In each of theses phases, they suggested to consider some quality metrics combining with the process decisions. They divided the model level into four parts, the first part is technical debt identification \& assessment where they suggested mine the repositories (Issue tracker and revision tool) identifying code and architecture smells and finding frequent changes in the software artifacts, then to record and save the data in the database. They used a specific form to collect the information on technical debt item. This form differs [43] of the form we used[41, mainly because of the form relates the debt with architectural and code smells, and in our model we did not consider $i t$. Comparing with our model we first suggest to discuss the technical debt metaphor, then the teams have to identify evidence of technical debt which includes code and architectural smells but it is not limited to. Subsequently, we recommend to analyze the historical data to collect the technical debt evidence. So, to identify the technical debt items we register the data on boards and tools. The second part in their model is Monitoring, where they select an appropriate debt item fields for technical debt values, the visualization of the debts, monitoring over releases and to make the decision whether the debt has to be paid - by refactoring - or the debt has an acceptable level and still be monitored. To visualize the debts they suggested a dedicated dashboard, to conduct the interview with the manager and to develop a risk mitigation strategy to be used if the technical debt reaches the ceiling. In our model, we consider to monitor as a part of the characterization activity, when the technical debt list is analyzed, each debt is measured - e.g., by rank - the debt impact is considered and these debts are being monitored and prioritized according to the metrics defined for each project. 
In the quantification \& prioritization part they quantified the debt to define a cost-benefit analysis. After the analysis of the technical debt impact, they determine the benefit and analyze the benefit-cost (e.g., less effort of refactoring), this cost is based on determining the cost, risk and estimate effort. As the cost is calculated, the debt is prioritized by the refactoring cost based on the ROI formula - ROI = (Saving Effort and Less Impact of Proposed Refactoring/Effort of Proposed Refactoring). In our model, we quantify the debt measuring them according to the important metrics for each team/project (e.g. risk, cost). Thus, we measure the items by tools and teams analysis to identify the cost and impact. After that, we discuss it considering the tools reports, prioritizing what debts can be paid. This prioritization is organized by the predefined template created by each team, once in our studies, we have identified that the relevance of each metric varies depending on the context of each team/project. The last part of their model is the repayment, since they have the items selected in the prioritize step, following the predefined rules, they decided to pay or not the debts. Once they choose to pay, they can pay off for short-term (Adding the items to current backlog) or pay off for long-term (Holding the items for next backlog/releases, to pay off by refactoring). In our model, when we have the technical debt list prioritized we suggested to the teams discuss and define whether technical debt will be paid off or not, or a new solution will be developed. After that, the items are put in the backlog to be paid by refactoring or as a Sprint task. When they consider putting the item in the backlog to be paid in a long-term, it is important to consider that new items can be inserted and identified in the code, and must be compared with this items to ensure that they still have the priority.

To summarize, they followed a different process to manage the debts but consider the most of activities we consider in our model, such as identification, monitoring, prioritization, and repayment. Another difference is that in our model we consider the debt cost during the characterization activity, where we also monitor the debts. They first monitor and then categorize by the cost of the refactoring. After that, they prioritize by ROI and then selected the refactoring can be paid.

\subsubsection{Conceptual Model of Technical Debt}

Avgeriou et al. [4, developed a recent conceptual model of technical debt. This model creates a map to show the relation with technical debt items and some software development aspects. We can observe that once the focus of our model is the activities of technical debt management in the Avgeriou et al. model they emphasize the properties, artifacts and the elements related to the technical debt item, as we can see in Figure 7.9

In the Izurieta et al. report [49] is highlighted that the Avegriou et al. [4] model summarized the elements related to technical debt but does not capture the activities to manage technical debt, being the focus of the model created in this research and therefore complementary to the model of Avegriou et al. Regard to the technical debt item, the Avgeriou et al. 44 model showed that once a technical debt item is inserted it is caused by some reasons such as decision, schedule, process. In our model, we also identify that the causes can be influenced by some motivations such as business decision, need for change, among others. Sometimes the motivation and the cause can be the same, but in other times the root of the cause is the result of some invisible motivations. Identifying this motivation can help the teams to organize some actions to mitigate the recurrence of this type of debt, such as training in specific technology.

The Avgeriou et al. model showed that a technical debt item is connected to some software attributes, such as code, test, etc. Using our model the teams can create a map with the location of the technical debt list, so, they can see what artifact of the software are the most impacted by the technical debt items. In fact, the technical debt we investigated directly impacted code, team and test. Additionally, our model helps the teams to show all the impacts of the debts, and not just the directs impacts as code. Because we emphasize that it is crucial considering the other aspects of technical debt management as motivations of the causes and the influence of the context.

The Avgeriou et al. model considered the consequences of the technical debt that affect the value of the software, as loss of quality. Complementary our model, in the impact branch they suggest considering the technical debt impact in the characterization activity to manage the debts. The 
Figure 7.9: Conceptual Model of Technical Debt

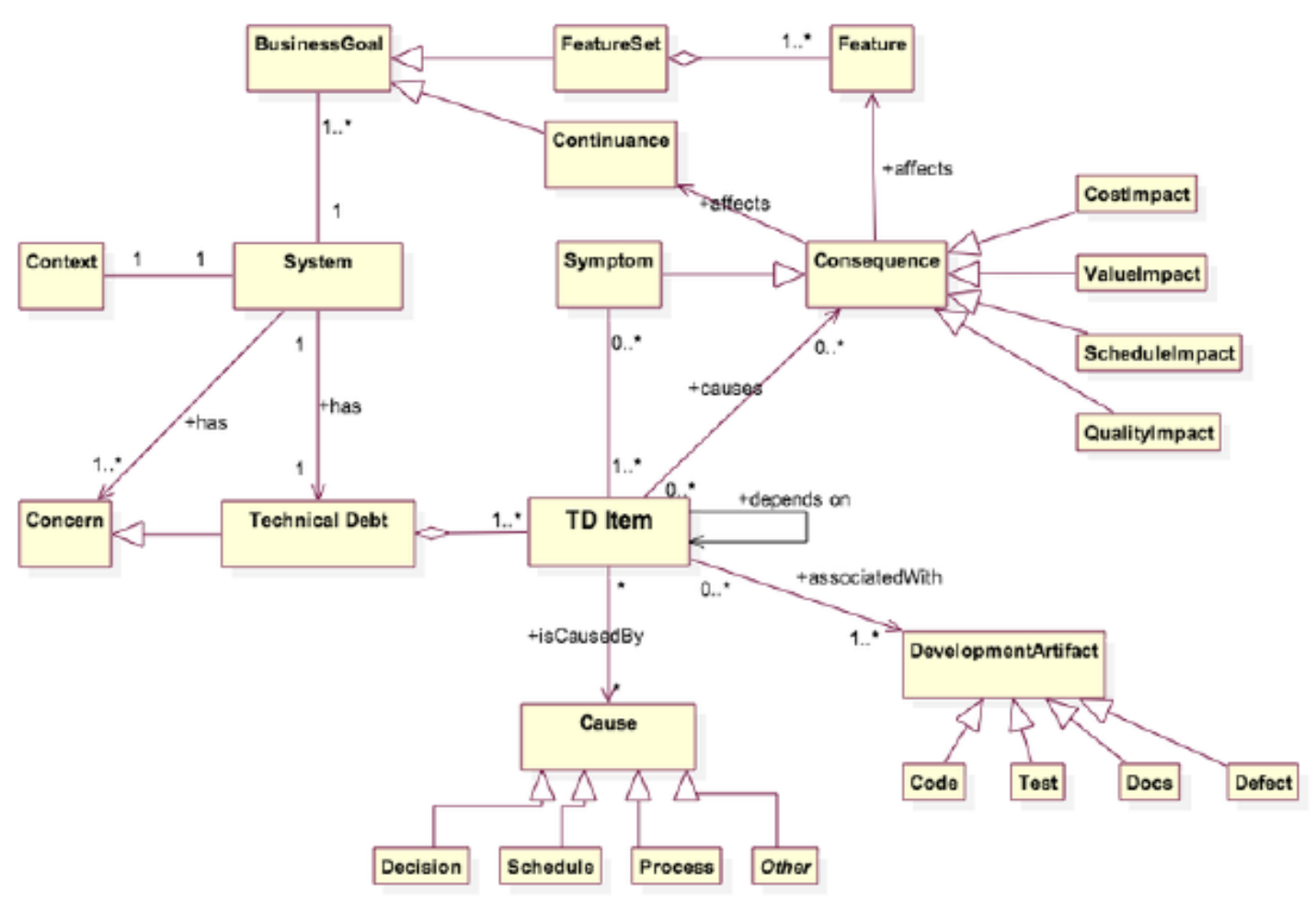

Source: [4]

Avgeriou et al. discuss the cost of technical debt composed of interest and principal. In our model, we defined the cost as one option to measure the debts and we defined as a metric to consider when the teams would create a rank to organize the technical debt list. They also consider that technical debt can have a symptom, which in our model we consider as a evidence and suggested some approaches, tools, and techniques to follow to identify and analyze the debt evidence. In both model we consider the context, but they connect the context with system and we precisely consider context to identify the influence on technical debt item and their causes. They created a cycle where the technical debt consequences affect a feature, then, this feature affects the business goal, that affects the system and the system has technical debt items. We consider that the technical debt impacts the business in a short-term because for example delivery delay, also in a long-term because of the effect in the feature. Besides that, many times a business decision was the reason that originated the technical debt, the root cause. Finally, as they said in the Avgeriou et al. [4] study, the model does not capture causes and the activities to manage technical debt. Considering the previous discussion our model is complementary to their model precisely focusing on the technical debt activities, causes and adding techniques, approaches, decision-making, activities and a process to consider for technical debt management.

\subsubsection{Technical Debt Management Framework}

Huumo et al. [109] investigated eight software development teams to understand how they deal with technical debt. In this study, they discuss each technical debt management activity. Although they did not create a conceptual model for technical debt management, they created a framework for technical debt management, because of that we compare this study with our model.

In the framework they considered eight activities for technical debt management: repayment, prevention, representation/documentation, identification, measurement, monitoring, communication and prioritization. For each of these activities, they created a level: practices/tools for activity, responsibility for activity, (Level 1) unorganized, (Level 2) received and organized, as we can observe 
in Figure 7.10

Figure 7.10: Technical Debt Management Framework

Table 5

TDM framework

\begin{tabular}{|c|c|c|c|c|c|c|c|c|}
\hline $\begin{array}{l}\text { TDM } \\
\text { activity/TDM } \\
\text { levels }\end{array}$ & TD repayment & TD prevention & $\begin{array}{l}\text { TD } \\
\text { representation/ } \\
\text { documentation }\end{array}$ & TD identification & TD measurement & TD monitoring & $\begin{array}{l}\text { TD } \\
\text { communication }\end{array}$ & TD prioritization \\
\hline $\begin{array}{l}\text { Organized (Level } \\
\text { 3) }\end{array}$ & $\begin{array}{l}\text { Continuous } \\
\text { repayment with } \\
\text { monthly } \\
\text { assigned } \\
\text { percentage of } \\
\text { the development } \\
\text { tasks. }\end{array}$ & $\begin{array}{l}\text { Mandatory } \\
\text { prevention } \\
\text { practices used } \\
\text { by the team. } \\
\text { Continuous } \\
\text { practice during } \\
\text { development. }\end{array}$ & $\begin{array}{l}\text { Documentation } \\
\text { is a mandatory } \\
\text { practice in } \\
\text { development, } \\
\text { Issues are } \\
\text { documented in a } \\
\text { separate TD } \\
\text { backlog. }\end{array}$ & $\begin{array}{l}\text { Continuous } \\
\text { identification } \\
\text { conducted } \\
\text { manually and/or } \\
\text { with tools } \\
\text { during } \\
\text { development. }\end{array}$ & $\begin{array}{l}\text { Continuous } \\
\text { measurement } \\
\text { during } \\
\text { development. } \\
\text { Data analysis } \\
\text { (various data } \\
\text { used (e.g. } \\
\text { quality. } \\
\text { performance)). } \\
\text { Assisted with } \\
\text { tools. }\end{array}$ & $\begin{array}{l}\text { Continuous } \\
\text { monitoring } \\
\text { during } \\
\text { development } \\
\text { with various } \\
\text { data (e.g. quality. } \\
\text { performance). } \\
\text { Tools used to } \\
\text { support. }\end{array}$ & $\begin{array}{l}\text { Continuous } \\
\text { discus- } \\
\text { sions/meetings } \\
\text { about TD issues } \\
\text { with all the } \\
\text { necessary } \\
\text { stakeholders } \\
\text { involved. }\end{array}$ & $\begin{array}{l}\text { Prioritization } \\
\text { conducted } \\
\text { continuously } \\
\text { during } \\
\text { development. } \\
\text { Prioritization } \\
\text { follows a specific } \\
\text { method or } \\
\text { model. }\end{array}$ \\
\hline $\begin{array}{l}\text { Received (Level } \\
\text { 2) }\end{array}$ & $\begin{array}{l}\text { Repayment } \\
\text { during normal } \\
\text { development } \\
\text { tasks and } \\
\text { previously } \\
\text { identified } \\
\text { repayment tasks, } \\
\text { Repayment } \\
\text { conducted based } \\
\text { on current } \\
\text { needs. }\end{array}$ & $\begin{array}{l}\text { Optional } \\
\text { prevention } \\
\text { practices, Not } \\
\text { mandatory to } \\
\text { use, but } \\
\text { recommended, } \\
\text { Conducted based } \\
\text { on current time } \\
\text { constraints. }\end{array}$ & $\begin{array}{l}\text { Documentation } \\
\text { an optional } \\
\text { practice, but } \\
\text { recommended. } \\
\text { Issues } \\
\text { documented in a } \\
\text { general } \\
\text { development } \\
\text { backlog without } \\
\text { TD id. }\end{array}$ & $\begin{array}{l}\text { Identification } \\
\text { optional during } \\
\text { normal } \\
\text { development, } \\
\text { Conducted based } \\
\text { on current time } \\
\text { constraints. }\end{array}$ & $\begin{array}{l}\text { Measurement an } \\
\text { optional } \\
\text { practice, } \\
\text { Measurement } \\
\text { done with } \\
\text { simple data } \\
\text { (number of TD } \\
\text { issues) from } \\
\text { development, } \\
\text { and the data nor } \\
\text { necessarily used } \\
\text { for other } \\
\text { activities. }\end{array}$ & $\begin{array}{l}\text { Monitoring } \\
\text { based on simple } \\
\text { data (number of } \\
\text { TD issues). } \\
\text { Conducted } \\
\text { occasionally. }\end{array}$ & $\begin{array}{l}\text { Discussions/ } \\
\text { meetings } \\
\text { organized only } \\
\text { with some } \\
\text { stakeholders. }\end{array}$ & $\begin{array}{l}\text { Prioritization } \\
\text { based on } \\
\text { hunches and } \\
\text { rough } \\
\text { estimations } \\
\text { based on } \\
\text { previous } \\
\text { experiences. } \\
\text { Prioritization } \\
\text { done in a simple } \\
\text { way without any } \\
\text { specific model. }\end{array}$ \\
\hline $\begin{array}{l}\text { Unorganized } \\
\text { (Level 1) }\end{array}$ & $\begin{array}{l}\text { Repayment not } \\
\text { conducted at all } \\
\text { or only when it } \\
\text { is not possible to } \\
\text { avoid the issue } \\
\text { any longer. }\end{array}$ & $\begin{array}{l}\text { Prevention not } \\
\text { assigned as part } \\
\text { of the } \\
\text { development } \\
\text { practices. } \\
\text { Conducted only } \\
\text { occasionally. }\end{array}$ & $\begin{array}{l}\text { Documentation } \\
\text { not part of } \\
\text { development, } \\
\text { Issues are left in } \\
\text { developers' own } \\
\text { minds and notes. }\end{array}$ & $\begin{array}{l}\text { Identification } \\
\text { practices not } \\
\text { assigned as part } \\
\text { of development, } \\
\text { Conducted only } \\
\text { when issues } \\
\text { occur. }\end{array}$ & $\begin{array}{l}\text { Measurement } \\
\text { not part of } \\
\text { development } \\
\text { practices. }\end{array}$ & $\begin{array}{l}\text { Monitoring not } \\
\text { part of } \\
\text { development } \\
\text { practices. }\end{array}$ & $\begin{array}{l}\text { TD not a topic in } \\
\text { discus- } \\
\text { sions/meetings } \\
\text { and of ten } \\
\text { handled only in } \\
\text { coffee table } \\
\text { discussions. }\end{array}$ & $\begin{array}{l}\text { Prioritization not } \\
\text { conducted, and } \\
\text { decisions done } \\
\text { without } \\
\text { reasoning or } \\
\text { discussions. }\end{array}$ \\
\hline $\begin{array}{l}\text { Responsibility } \\
\text { for activity }\end{array}$ & $\begin{array}{l}\text { Development } \\
\text { team, software } \\
\text { architect(s) }\end{array}$ & $\begin{array}{l}\text { Development } \\
\text { team, sof tware } \\
\text { architect(s) }\end{array}$ & $\begin{array}{l}\text { Development } \\
\text { team, software } \\
\text { architect(s) }\end{array}$ & $\begin{array}{l}\text { Development } \\
\text { team, software } \\
\text { architect(s) }\end{array}$ & $\begin{array}{l}\text { Software } \\
\text { architect(s). } \\
\text { team manager }\end{array}$ & $\begin{array}{l}\text { Software } \\
\text { architect(s). } \\
\text { team manager }\end{array}$ & $\begin{array}{l}\text { Development } \\
\text { team, software } \\
\text { archirect(s). } \\
\text { team manager }\end{array}$ & $\begin{array}{l}\text { Software } \\
\text { architect(s). } \\
\text { team manager }\end{array}$ \\
\hline $\begin{array}{l}\text { Practices / tools } \\
\text { for activity }\end{array}$ & $\begin{array}{l}\text { Refactoring. } \\
\text { redesigning. } \\
\text { rewriting }\end{array}$ & $\begin{array}{l}\text { Coding } \\
\text { standards, code } \\
\text { reviews, } \\
\text { Definition of } \\
\text { Done. }\end{array}$ & $\begin{array}{l}\text { Technical debt } \\
\text { backlog/list, } \\
\text { Documentation } \\
\text { practice, project } \\
\text { management } \\
\text { tool (JIRA, Wiki) }\end{array}$ & $\begin{array}{l}\text { Time reservation } \\
\text { for manual code } \\
\text { inspection, Use } \\
\text { of code analysis } \\
\text { tools } \\
\text { (SonarQube, } \\
\text { CheckStyle. } \\
\text { FindBugs). }\end{array}$ & $\begin{array}{l}\text { Data from } \\
\text { measurement } \\
\text { tools } \\
\text { (SonarQube) and } \\
\text { data from } \\
\text { project } \\
\text { management } \\
\text { tools (JIRA. } \\
\text { Wiki). } \\
\end{array}$ & $\begin{array}{l}\text { Monitoring tools } \\
\text { (SonarQube). } \\
\text { Project } \\
\text { management } \\
\text { tools (JIRA. Wiki) }\end{array}$ & $\begin{array}{l}\text { Specific TD } \\
\text { meetings, TD } \\
\text { included in } \\
\text { discussion } \\
\text { topics. }\end{array}$ & $\begin{array}{l}\text { Cost/Benefit } \\
\text { model, Issue } \\
\text { rating }\end{array}$ \\
\hline
\end{tabular}

Source: [109]

In the repayment, they listed that a team in an organized level do a continuous repayment and achieve the predefined percentage by the team. We consider this activity in our model as part of the characterization process. They set as the responsible for this activity the development team and software architect(s), as a practice they suggested refactoring, redesigning and rewrite.

In the prevention activity, they suggested that teams in the high level follow mandatory prevention practices and continuous practices during development. As a practice, they suggested coding standards, code reviews, and definition of done.

In the representation/documentation activity, they listed that teams in the organized level have it as a mandatory practice. They suggested for it a list of technical debt in the backlog, documentation practice and management tool (Jira, WIKI). In our study, the teams also used a tool to register and document the information on the technical debt item. We did not consider it a particular activity, instead of, we suggested that the information about the debts have to be collected from the moment of the identification to the moment of the payment, including if they decided to not pay the debt.

In the identification activity, they related that the practitioners with organized level, continuously identify manually or with the tools during the development. As a practice, they suggested that 
the teams can save some time for manual code inspection, and using code analysis tools(SonarQube, FindBugs and CheckStyle). In our model, we suggested also using manual, and automated form, including tools (Jira, Eclemma, FindBugs, CodeClimate and SonarQube), and boards (Scrum board and technical debt board).

To measure and monitor the debts, according to them, an organized team continuous measure considering and analyzing data from different metrics such as quality performance, including doing it by tools (Jira, Wiki, and SonarQube). Until this activity, they recommended as responsible the development team and the software architect(s). In the measure and monitoring activity they suggested the software architect(s) and the team manager. In our model, to measure the technical debt items we also recommend the use of tools (Jira, SonarQube) and we created a rank for the technical debt list and discussed it in the meetings, e.g., Sprint Planning meeting. We also suggested some metrics to organize the rank. To monitor the debts we also recommend manual and automated approaches with tools and boards, discussing in a meeting, adding graphics, plugins, alerts, TV Screen and practices as continuous inspection, pair programming and code review.

For the communication activity, they proposed in an organized level discussions/meetings about technical debt issues with all the stakeholders involved. The responsibility includes development team, software architect(s) and team manager, as a practice, they suggest discussion in meetings. In our model, we do not consider communication as a specific activity, since we suggested that teams must discuss the technical debt items during all the process for technical debt management.

Finally, in the prioritization activities, they recommend that a team in a high level continuously prioritize following a specific method or model and using practices such as cost/benefit model, issue rating. As the responsible, they suggested the software architect(s) and the team manager. In our model we consider that to prioritize the debts it needs to analyze his impact, to measure considering metrics as costs, then following a predefined template organizes the prioritization. This prioritization also depends on the metrics the teams decided that is a priority for each project. We consider repayment as a payment activity, where the practitioners must decide to pay and then consider the debt in a backlog to pay in a sprint as one more task of the sprint, or in a specific day turned to improve the software quality, such as refactoring day. The Huumo et al. [109] framework is complementary to our model, both cover some things that are not covered by the other.

\subsubsection{Managing Architectural Technical Debt: A Unified Model}

Besker et al. 9] created a descriptive model focus on architectural technical debt, as we can observe in Figure 7.11 .

This model has four main group: Importance of ATD (Architectural technical debt), ATD Checklist, ATD Impediments, ATD Management. In each of these groups, they listed the related aspects and how they are connected. In ATD importance they listed aspects as an impact on overall software life-cycle.

In ATD Checklist they list five aspects of categorization such as dependency violations. In ATD Impediments they divided in Challenges and Negative effects, for example as a challenge they list detection and as a negative effect of it, maintenance and evolvability.

In the ATD Management, they divided into ATDM Activities, methods and refactoring. In this part, they cited Identification, Measurement, Prioritization, Repayment, and Monitoring. For this activities they suggested as a methods/tools, measuring, tracking, evaluating and to do a refactoring they list four dimensions that have to be considered. In the ATD model, the focus is architectural technical debt and our model the focus is to manage all type of technical debt. However, the Besker et al. 9] model they also consider as management activities the activities that we used in our model: identification, measurement, prioritization and monitoring. They suggested using tools to management activities but also said that tools currently available cannot identify all types of architectural technical debt. As we identify in our studies other types of technical debt items mainly that connected and related to a business rule. One interest thing is that they emphasize that sometimes is a better option to pay the interest rather than do a refactoring in case of architectural technical debt. In the studies we conducted, many times the practitioners choose to do a refactoring 
Figure 7.11: Technical Debt Management Framework

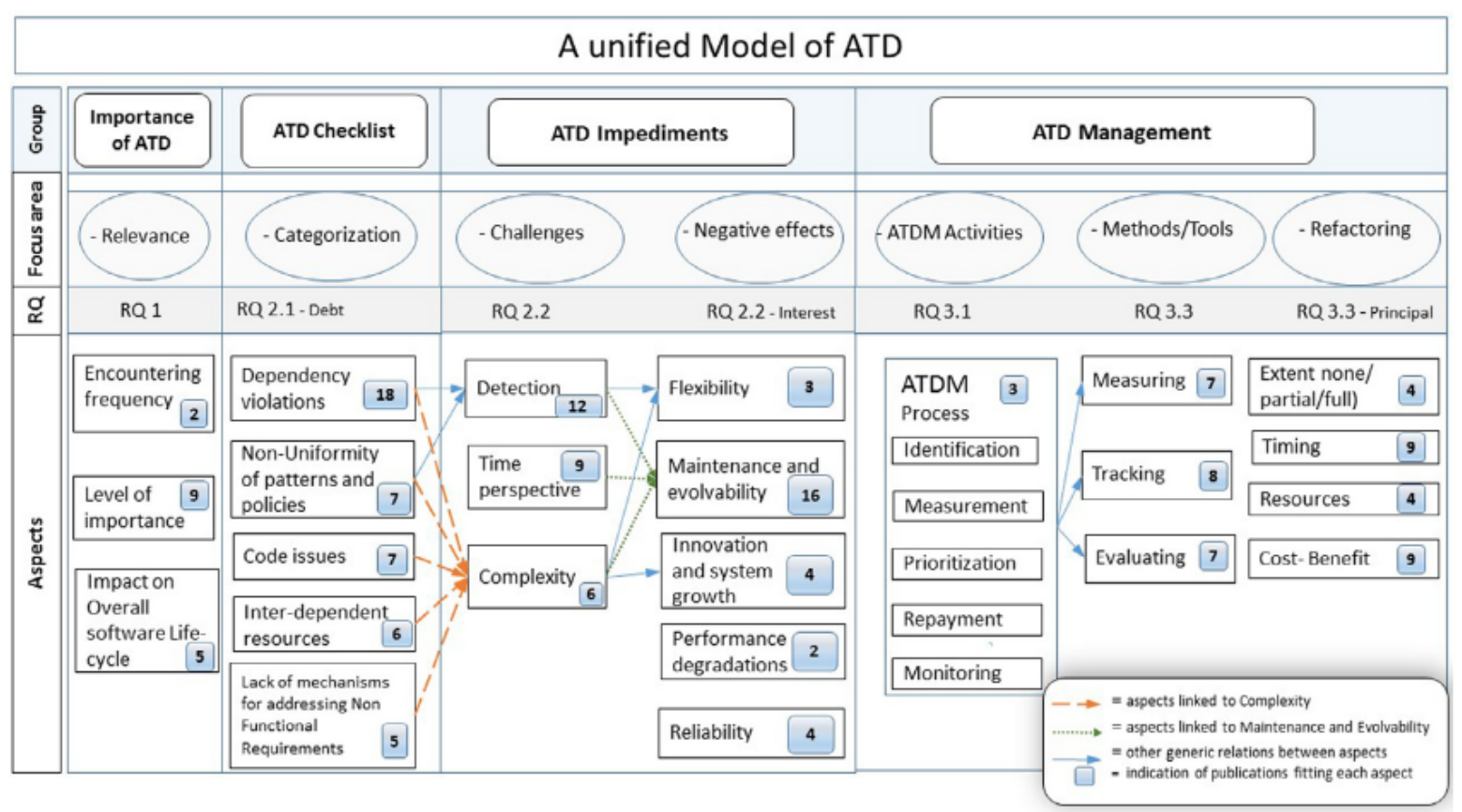

Source: [9]

as a solution to pay the debts. In summary, their model also suggested to consider other aspects to manage architectural technical debt rather than just the technical debt management activities, such as impact, negative effects, categorization, etc.

\subsubsection{Technical Debt Management Cycle}

Bill [21] suggested seven steps to manage technical debt in the Figure 7.12.

Figure 7.12: Technical Debt Management Cycle

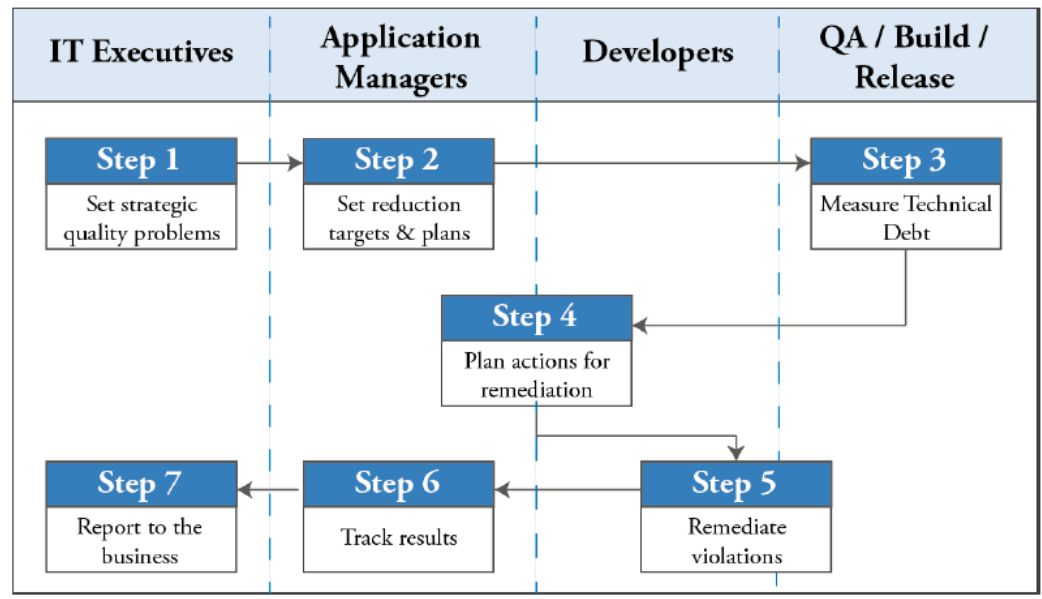

Source: 21]

The first step is the IT Executives to determine the quality priorities for each of the application services it offers to the business (Set strategic quality priorities). In our model, we suggested to the development team to determine which technical debt it is the most important to consider for each project/sprint. Besides, Bill suggests that the application managers will need to translate the structural (establish reduction targets and plans), defining which pattern represents the quality needs to the development team. After, he suggested to measure technical debt in many moments 
of the software life cycle, for instance, with static analysis tools (Measure Technical Debt), as we recommend and use in our studies. Next, in the plan remediation actions the application manager and development team can use the structural quality analysis from the previous release to prioritize a list of violations for paying some technical debt and achieving the main objectives of the quality. In to remediate violations step, they suggested that the development team have to pay some debt as a part of their 'normal' development or maintenance activities, these remediation activities can be things such as refactoring. To pay some debts by refactoring is one of our suggestion. As a next step they recommend to track results, should compare the results of technical debt remediation effort to established targets and share them with the developers. Also, the application manager and IT executives can review the progress periodically. Finally, they proposed report to the business, the status of technical debt. It can complement our model.

They divided this model by types of the teams and their responsibilities, such as the QA team - to measure the debts-. In our model, we did not categorize each activity for technical debt management by team. Because our focus is on how to manage technical debt independently of who will conduct each activity. However, we also recommend that the business teams and the customer need to be in line with the technical teams to achieve better results.

\subsubsection{Summary of the Comparison of the Models}

The models from the previous subsection are directly connected to our study because they are models or frameworks focused on the technical debt management activities. Several studies discuss technical debt management but are not models or frameworks. Most of them discuss specifically aspects, activities, techniques or suggestions for technical debt management. In particular, three studies are secondary studies and they summarized and categorized most of the studies in the area of technical debt.

Sánchez et al. [31 conducted a systematic mapping to identify the techniques and methods for technical debt management, with focus on the architectural debt. They organized a framework listing the elements for technical debt management, as we can see in Figure 7.13 .

Figure 7.13: Framework for the Elements for Technical Debt Management

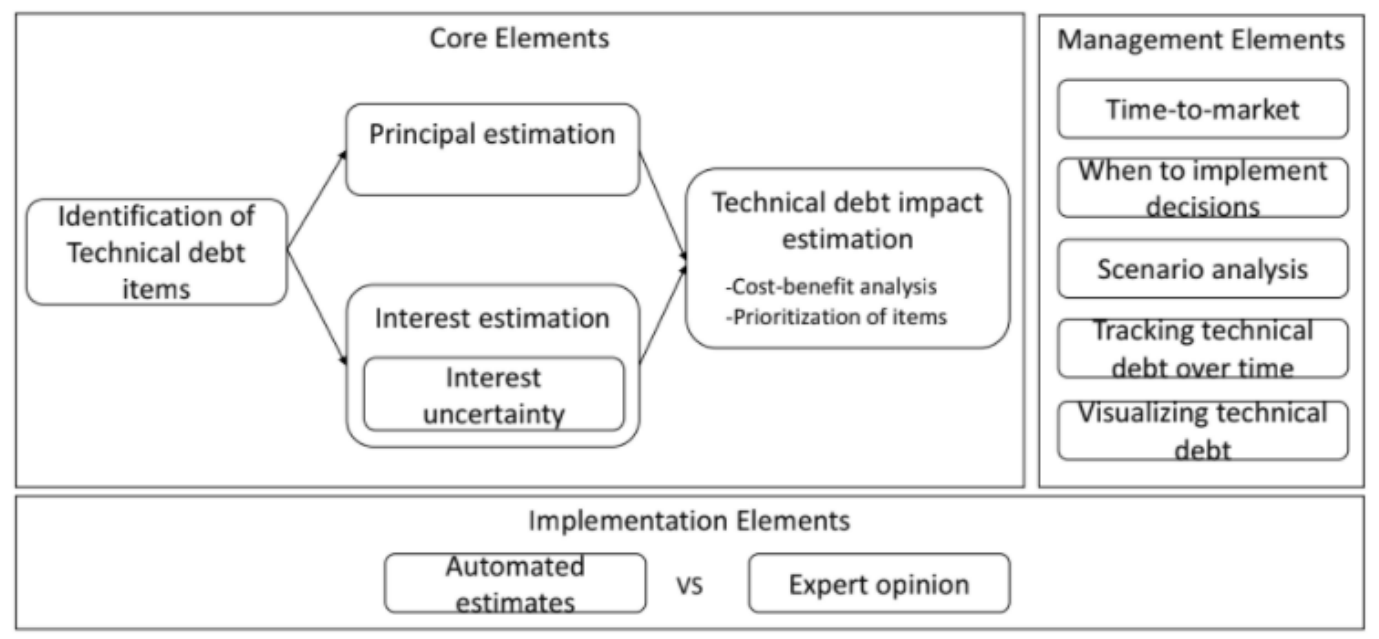

Source: [31]

They divided the framework into core elements, according to them these elements represent "...concrete requirements to achieve in order to be able to manage technical debt.". They divided the elements into three groups: implementation elements - focus on estimating the items of technical debt on the system (e.g. principal estimation)-, the management elements - to define what is necessary to identify the debts (e.g., visualizing technical debt) - and the implementation elements (e.g., expert opinion). In the core elements, they identified the technical debt and estimated the cost and impact. As a management elements they suggested to track the debts and make them visible. Also, 
they recommend considering aspects of the technical debt as time-to-market, when to implement the decisions and create the scenarios analyses of the debt and its implication. Furthermore, in the implementation elements group, they suggest balancing between automated estimates and expert opinion. All this item we consider in our model, but we categorize each item in a specific area of the technical debt management and organize them in the process of the debt management, for example, tracking and making the debts visible in the identification and monitoring activities.

Yin et al. [107] did a systematic mapping on technical debt and its management. The study was conducted in 2014 and published in 2015, so it covers most of the studies until there. They classify technical debt according to the metaphor, causes, effects, uncertainty, and properties. In addition, they identified as a technical debt management activity: identification, measurement, prioritization, prevention, monitoring, repayment, representation/documentation, and communication. In our model we consider as technical debt management activity: identification, measurement, prioritization, monitoring, characterization and payment. They gave many examples of tools the teams can use for technical debt management and classify these tools according to each activity the tools can attend. They also consider the tools we used in our study, such as SonarQube, PMD, Checkstyle, but they do not consider the tool Code Climate that the teams used in the Classroom studies. They suggested some approaches to prioritize and monitor and repay the debts. They consider as a repayment approach, refactoring and rewriting - we consider to payment - and they added other options as automation, bug fixing, etc.

Alves et al. 22 characterized the technical debt types, suggesting many indicators that can be used to identify technical debt items. Also, they recommend management strategies and visualization techniques that can be used for technical debt management and identification. They identified fifty types of technical debt, this list can help teams to start the discussion on technical debt identification to define which debt type the project could have, to choose the most critical. So, they can consider as a first step to identify the debts, also to create a list of categorization by type. In addition, they have a table with the list of indicators that teams could use to identify each type of technical debt. This list can complement parts of our model, where the teams collect evidence of technical debt item and monitor the type of items to consider in their projects. They list six techniques to technical debt visualization. In relation to technical debt management, they discuss five strategies but list twenty approaches for technical debt management.

Most of these researches about technical debt management were focused in a specific part of the process of technical debt management. Considering our model to understand the concept of technical debt we can used the studies of Cunningham [?] [20, Brown et al. [12] [11, Theodoropoulos et al. [100], Allman [1], McConnel [61], Kruchten et al. [51], a recent definition of technical debt from the Seminar on Technical Debt of Avgeriou et al. 4. Also, several times the teams could not understand what is not a technical debt, the studies of, Bavani [6], Curtis [22, Kruchten et al. [52, Sterling [95. give some examples on it. In addition the study of Tamburri et al. 98] gives an example of the other type of debt, that can influence the technical debt incurrence, but it is not a technical debt.

The studies of McConnel [60], Fowler [35], Avgeriou et al. [4, and Izurieta et al. [49] can help teams to know about the technical debt characteristics. But if the teams need to register the debts its important to consider the technical debt properties, then they can follow the studies of Brown et al. [12], Guo and Seaman [42], Falessi et al. [30] and Li et al. [55].

For technical debt identification the teams can follow the suggestions of the studies of Sterling [95, Zazworka et al. [111], McConnell [60, Rubin [79, Bellomo et al. [8], Brown[12, Bavani 6], Eisenberg [26] and Zazworka et al. [110]. To classify the debts by type we followed the studies of Guo and Seaman [41] and Li et al. [55].

According to our model, once we identify the debt we recommend to identify the root causes and motivations of the technical debt incurrence, the studies of Brown et al. [11, Mcconnell [60], Sterling [95], Buschmann [13, Lim et al [57], Codabux and Williams [15], Spínola [93], Falessi et al. [30], Siebra et al. [89], Kruchten et al. [51], Martini et al. [59] and Rubin [79] report some reasons for this.

Other studies present the technical debt impact, as in the studies of Brown et al. [12] [11], Sterling 
[95], Buschmann [13, Highsmith [44, Allman [1], Siebra et al. [89], Curtis et al. [22], Lim et al [57], Bavani [6] and Neil [27].

Other approaches cited are about applied technical debt management focus to manage a specific type of technical debt, or to suggest a specific approach for it in a specific case, discussing aspects like decision-making. We consider the studies of, Seaman et al. [88] [87, Allman [1], Nord et al. [67], Neil et al. [27], Brown et al. [12], McConnell [60], Schimd [84], Sterling [95], Bavani [6], Dos Santos et al. [24, Li et al. [55], Bill [21], Letouzey [54, Holvitie and Leppanew [47] 46], Falessi and Voegele [29], Sandberg et al. [81], Izurieta et al. [48] [49], Oliveira et al.[70], Ramasubbu and Kemerer [76] [75], Lim et al. [57, Guo's [42], Huumo et al. [109], Vathsavayi and Systã [105] and Chicote [14].

Once the teams have identified the debts they should measure them. So it is possible to define which debt it is crucial to pay. For technical debt measurement we suggested the studies of Guo and Seaman [42] [41], Brown et al [12], Eisenberg [26] and Letouzey [54]. As soon, as the debt items are estimated the next step is organized the technical debt prioritization, the studies of Einsenberg [26], Nugroho et al. [68] and Letouzey [54] suggest some approaches to do it.

After identifying, registering and measuring the debts it is important to monitor these debts over time, for it when we started our study we follow the suggestions from the studies of: Guo and Seaman [41], McConnell [60], [12, Sterling [95] and Letouzey [54]. Also, we have some tools that can help for technical debt monitoring and measure, the studies of: Eisenberg [26, Holvitie and Leppanew [47, Ernest [28] and Li et al. [55] and Sanchez et al. [32] explain some of these tools.

The debts sometimes have to be payed off, for it the studies of: Allman [1, Guo and Seaman [42, Sterling [95], Allman [1, McConnel [60], Holvitie and Leppanew [47], Letouzey [54] discuss some approaches to guide the repayment.

The studies related above served as a base for our study, but as we previously reported, these do not encompass all technical debt studies. In the recent years, several studies were published and were not considered in this research since our goal was not to do a systematic review of the area. In the next sections, we are going to answer each research question and summarize the findings.

\subsubsection{Threats to the Validity}

In this study, we took some actions to mitigate the bias, and these actions can be best described in the following points:

- Bias due to the analysis and interpretation of the data: data were interpreted following the steps of the action research method. In each cycle of the action research we analyze and improve the model by each team. Lastly, we compared and merged the findings that were relevant and presented plenty of evidence in the results of both studies. This model was based on the suggestion made bu the researchers but also the team members that participated of the meetings to discuss each model evolution. If we had any doubt about a specific point we went back to the data and re-analyzed them. Also, we have the some version of the tools reports saved. After that, the results were read by the advisor and co-advisor and if they indicated some points to be re-analyzed, the researcher re-analyzed the data. . We recommend that these studies be replicated in other cases and the results compared before they could be generalized.

- Bias due to the context: It should be emphasized that the studies conducted in companies are aimed at solving the problems of the company and are the best option for those very specific cases. Therefore, the studies cannot be generalized either. We sought to develop them with the highest possible scientific rigor and we also sought to report the studies, and the motivations of each decision in details so that they can be replicated and tested in other cases.

\subsection{Chapter Summary}

This chapter describes the model for technical debt management. Suggesting several ways, activities, techniques, steps and tools to manage technical debt items. Also, the results are compared 
with the literature related studies. Six main models were found in the literature and compared with our conceptual model for technical debt management. The models have some complementary approaches that are discussed and suggested in order to technical debt management. Additionally, we cited the studies related to technical debt management we considered to developed this study, these studies include techniques, approaches, discussions and examples on technical debt identification, measure, management, monitoring, types of technical debt, technical debt characteristics and concept. 


\section{Chapter 8}

\section{Conclusion}

This chapter summarizes the main findings of this research that involves a Classroom study and two big Brazilian IT Companies. These studies combine literature studies, action research and case studies in nine academic teams and five business teams, all of which used agile methods. Bellow, we summarize the findings, answer each question, describe the main contributions, and suggest future works.

\subsection{Summary of the Findings}

The growing number of studies in the area of technical debt show that this area has been receiving attention due to the perception of its relevance. In these studies, we mainly contribute in how to deal with the technical debt items and how it can impact the software development teams. The results of the studies in the XP Lab 2013 and 2014 editions shows the impact on the teams once they were aware of the technical debt items. We have also identified the influences on teams with graduate and postgraduate students. The team members were always very excited about making technical debt items visible. In these studies, we validate the relevance of considering technical debt items to the teams. Mainly the teams improved their communication and thought more about the software quality, creating metrics and using tools to monitor the debts rather than inserting them into the code and forgetting about them.

In the Companies studies, we created and tested some approaches for technical debt management. In the first study at Company A we identified the impact on the project once a technical debt item was inserted in the code but ignored in the long-term. In addition, in this study, it was possible to observe where some technical debt items were originated, the reasons and causes that had motivated incurring them. Considering that the first study identified some technical debt items in the project and the significant impact of ignoring it. In the second study in Company A, the teams started to identify, monitor and manage the technical debt items following the process they already used for software development and management. A first version of the conceptual model was created to help teams to start considering technical debt without substantial changes in the process, technologies, tools and ways they still used to develop the software.

Throughout the studies in Company B, we considered technical debt in two agile teams that had a different level of maturity. During three action research cycles, the conceptual model for technical debt management was tested and improved based on the first version of the model developed during the studies we did in Company A. Aiming to facilitate the understanding and application of this model by software development teams, we created two examples of how this model can be applied using some data from the XP Lab studies and Company A studies.

The conceptual model for technical debt management resulting from these studies contributes to reducing the gap in the technical debt management area. It is important to emphasize that this model is based on the results of the tests performed in the studies and the data analysis, as it is a data-based model. We can see the last version of the model in Figure 8.1.

As a first step, we identified and analyzed some major changes in the code in three different 
Figure 8.1: Conceptual Model for Technical Debt Management

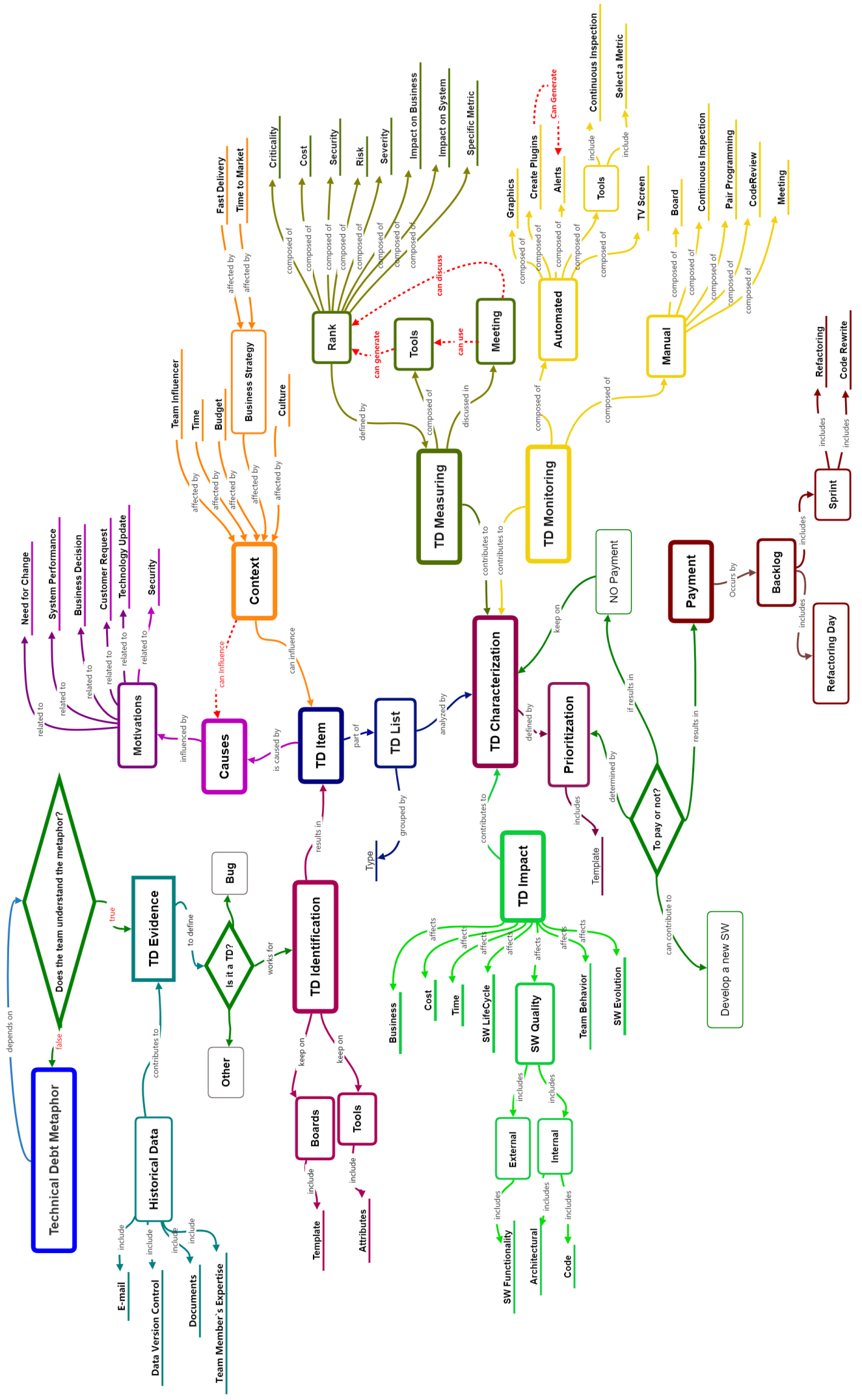

projects of Company A's teams. As a result, we identified technical debt items that impacted the projects. The main impact was on time, seeing as the project delivery was delayed more than 120 
days. The impact of the debts also included a high stress level of the team and losses for the business once the competing companies have delivered a more advanced, fast and efficient product to the market. After we identified the technical debt items and their impacts, we analyzed the reasons that motivated the incurrence of these debts. As a result, we created a model with the technical debt impact and motivations, highlighting the direct result of such technical debt items occurrences, as for example code rewriting.

In the second step, we started to consider technical debt in two agile software development teams at Company B, where we performed three action research cycles. The aim was to identify, monitor, and manage technical debt. For it, we started by doing a talk on technical debt and discussing with the teams strategies and ways to start considering technical debt. We defined as the main objective of this cycle to start activities aimed at identifying the technical debt items. We also started to discuss the second study at Company A, with the purpose of starting to identify, monitor and manage technical debt. In the study in Company A we started register the debts, monitor and define whether pay off or not the debts at the same time. The teams created a template to register the technical debt items in the Jira tool and listed them on Scrum board. They defined a technical debt as a type of story. The story technical debt had the fields: ID, name, description, responsible for the item's register, priority, related tests, stakeholder, who was assigned to pay the debt, criticality, technical debt interest, the probability of the technical debt resulting in interest, estimated time to implement it in hours, location in the source code, and the related task that generated the technical debt. The field interest could be filled with the number of points the team used to correct or maintain the technical debt item, while the probability could be filled with the measure: low, medium or high. They monitored the debts analyzing in the list of technical debt registered in the Sprint Planning meetings. According to the list and discussion, they decided whether pay off the technical debt and putt the item in the backlog or pay it during the sprint.

In the third step, we concluded the studies at Company A. As a result, we created the first version of the conceptual model for technical debt management. We also started to plan the second cycle at Company B. The aim was to focus on monitoring technical debt items. Since these two studies (Companies A and B) were being conducted at the same time, the forms of identification used in company B were suggested in Company A, and the monitoring and management techniques used in Company A were suggested and evolved in Company B. Moreover we started the Classroom studies in the XP Lab 2013 edition. The objective was to characterize the impact on the team when technical debt items are visible, based on team members' perceptions. The teams used the Jira tool to register the items.

In the fourth step, we finished the third cycle at Company B, where the aim was to begin the technical debt management and payment, which we monitored during previous cycles. In addition, we repeated the study at the XP Lab 2014 Edition, with the intention to characterize the impact on the team when technical debt items are visible, based on team members' perceptions.

As a final result, we created the conceptual model for technical debt management.

The model suggests techniques, approaches, and directions to help software development teams to manage the technical debt items. This model can also help teams to identify which decisions result in debts and what are their impact, thus contributing to the understanding of reasons that motivated the technical debt incurrence. This way the teams can strategically use the technical debt items to aggregate value to the business. Additionally, they can arrange talks, courses and training to mitigate the recurrence of those types of technical debt that have a greater impact on the project.

In the next sections we answer each research question. After, we present the contributions and future work.

\subsection{Discussion of findings by each research question.}

In the next sections, the findings are reported according to each research question. 


\subsubsection{RQ1 (Company A): Which decisions may motivate a team to incur tech- nical debt in agile software development projects?}

The analysis of the four cases in the first study at Company A shows that all the technical debt items analyzed were contracted motivated by a strategic decision. Sometimes because of a mandatory business decision - without discussion of the team. Another motivation was the technical changes that were based following an opinion of the one team member, an expert in a specific technology. And sometimes they used arguments as performance gain to do that change, but in fact, this was not the real reason for the change. The other reason was the team's knowledge deficiency in a certain technology. Where they believed that another option could be better, but did not know the new option very well. Another decision is related to fast delivery, where the teams choose to incur debt due to time. In one case, they decided due to data inconsistency, to centralize the recording of the data in a single system, but in fact, it generates more data inconsistency implying in many late deliveries. Finally, concerning the lack of analysis in a more detailed way for the market options versus the demand. But, for example, a lack of the understanding of the requirements could result in a technical debt. The teams highlighted that considering technical debt help the teams discuss more the tasks and the software quality. To summarize the decisions that resulted in technical debt were changes requested by the business team, time to market, fast delivery, software improvement needs such as performance and upgrading technologies versions based on the opinion of one team member.

\subsubsection{RQ2 \& RQ6 (Company A and B): What types of technical debt items did the agile software development team incur?}

In the study we did in Company B, the teams incurred mainly in test debts. But, they purposely chose to monitor only this type of debt, probably their projects had many other types. In the Company A, they also had architectural technical debt, technical debt related to performance and documentation. Also, the teams emphasize that tools with static analysis of the code quality could help them to identify the debts. But that technical debt such as business rule, design, usability, among others directly related to the specific business or technology is still hard to identify by tools.

\subsubsection{RQ3 (Company A): What is the impact on an agile software development team of the technical debt in their project?}

The team members related many impacts during the project because of the technical debt incurrence. One is the direct impact on business for instance because the competitor released on the market a more advanced product, time and cost. The studies related impacts affecting the team motivation and generate a high-stress level. Thus, the customer also was unsatisfied with the solution. The impact in the case of the jQuery version upgrade was more than $\mathbf{1 2 0}$ days late. In the other three cases also the technical debt items impacted the project delivery in over a month. Also, they related that the team had to work at ahigh-pressure level and because of that it was very stressful. They related that many other new features were developed and could not be placed in the production environment since they did not have a functional front-end due to the upgrade. Also, the code of a project became too complex to maintain that it became impossible in a determined moment that a new solution had to be developed. Other times they had to adapt the solution due to the code complexity and/or technology restrictions. In the case where technical debt resulted in data inconsistency, much information about infrastructure estimates were lost.

Technical debt in most of the cases will generate an impact, the team should monitor and manage the debts to mitigate it. 


\subsubsection{RQ4 \& RQ7 (Company A and B): Which techniques and tools can agile software development teams use to identify, monitor and manage techni- cal debt?}

Two agile teams were followed in Company B, once we did action research, each strategy defined in these cases was decided in joint with the team leaders. In both of cases, the teams started to consider technical debt following the same tools, technologies they still used, just with a small adjustment in the team process and in the form they still used to registered issues. For it, they put the technical debt items in the Scrum board near to the tasks, but in a specific place for the technical debt items. Also, a reminder was on the board listened to the fields that should be filled in each item registered in the Jira tool and on the Scrum board. Additionally, they used TV Screen graphs to show how many technical debt items were inserted in the project by time. In the Jira tool, they created one more type of issue called technical debt. They discussed the technical debt list during the sprint planning meetings and they decided whether to be paid. As a first strategy to pay the debt, they defined just one team member to do it. However, they changed for everyone should pay the debts, since when only one was paid it would be comfortable for the other team member's to insert more debts in the code. Also, the paying member could no longer afford only to pay debts. They used the SonarQube and PMD to monitor the technical debt items and they developed a plugin integrate to the Eclipse framework to monitor the code quality. The teams also used installed a Sonar plugin on the Jenkins ${ }^{1}$ server to trigger a parsing in the code after the daily building. They also created a plugin adapted to their needs. This plugin used other code quality analysis tools: Checkstyle 4, Coverage, and PMD.

In the Company A, the teams used the Jira tool to register the items and the Scrum board to make them visible. They also used the SonarQube tool to monitor the debts.

In the Classroom studies they used SonarQube, EclEmma, CodeClimate, JUnit framework and the framework RSpec to identify and monitor the debts.

The techniques they used to monitor and manage were: registered the debts in Jira tool and boards (e.g. Scrum board, Technical Debt board). Discussing the cost and payment in the meetings, as in the Sprint Planning meetings. Defining the way to pay it off, as in a Refactoring day, or putting in the backlog of the Sprint.

\subsubsection{RQ5 \& RQ8 (Company A and B): What is the impact on the software development process when the agile software development team considers technical debt items?}

The team members emphasized that the impact on the process was not relevant once the teams follow the same process the still used to manage and develop the software. They considered that the impact was small given that the benefits of considering technical debt. When we asked them if they have to spend to much time to identify the debts, they said that as was the same process to register the bugs, they did not see big changes adding one more type of item to consider. But, in all of the studies, we follow the same process they used to implement the tasks, manage the project and correct the bugs. Technical debt was just one more type to consider. The big challenge was to save time to pay the debts because it includes convincing managers and technical leaders to prioritize this in the sprint.

\subsubsection{RQ9 (XP Lab): What is the impact on the team when technical debt is explicitly considered?}

All the team members emphasized the importance of the project and the team to consider technical debt. Because the team communication was improved, all of them thought about quality, not just specific members. The culture of the team improved and in addition, the team believed that it was easier to show the level of the technical debt impact to the customers

\footnotetext{
${ }^{1}$ https://jenkins.io/
} 
business, showing that it is possible to invest some time improving the quality. Furthermore, the teams used the debt registration template and concluded that it was simple to use. In addition, they stated that listing debts in a chart had benefits such as quickly identifying the trend of code quality, giving visibility to all debts, being aware of software problems. Problems that were inserted and forgotten, in a simple way could be evidenced and treated strategically. Additionally, using the board was already a routine activity for agile teams, so there was no major change in the way they work.

In the Classroom studies the main results emphasize the Extreme Programming values and help the teams to promulgate values as communication with team members and with team members and the customer, respect with each other in the team, the courage to change and feedback to improve the software continually. All the team members strongly recommended considering technical debt in software projects because they considered it so important. They also highlighted that they will consider technical debt in the next projects for reasons such as: if they ignore it could be a future problem, to know how are the software quality and better understand what they have to do to improve it and because they could improve their skills as programmers and how to evaluate third-party code.

In the next section, we related the main contributions of this research.

\subsection{Contributions}

This section presents the summary of the contributions of this research. As explained before, the main goal of this study is to suggest a model to identify, monitor, and manage technical debt as well as exploring its impact in agile teams once they are aware of the technical debt concept. Thus, the agile software development teams can use it to deal with technical debt in their projects. The main contributions of this research can be summarized as:

- We present the state of art in the technical debt management area and we did an analysis of several studies on technical debt identification, monitoring, payment, prevention, measuring, prioritization, etc. In Chapter 2 we have related several studies on technical debt management, identification, monitoring, payment, prevention. We also provide evidence of the empirical studies on technical debt in different agile development environments.

- A conceptual model for technical debt management. In this model we consider aspects of technical debt such as the reasons, motivations and the impact of technical debt on agile software development projects and teams. In addition, we show steps, approaches, techniques, and tools that the agile software development project teams could choose to identify, monitor, measure, prioritize and manage technical debt in their projects. Additionally, we did a comparative analysis of our conceptual model with similar models from the literature. Furthermore, the model with its current level of abstraction can be used as a guide for companies to define strategies for identification, monitoring, measurement and technical debt management.

- During our empirical studies, we always tried to let the developers use their current tools, adding whenever possible the technical debt awareness to their usual environment.

- The insights and results presented after the application of the model can help the teams to understand the challenges of dealing with technical debt.

In the next section, we suggest the future works.

\subsection{Future Works}

Throughout the development of this research, some gaps and opportunities for new research became evident. Below we describe each one of them. 
- Technical debt impact: in future work it is important to verify the influence of considering technical debt on the team in the long term, especially concerning speed and code quality. It is also important to create ways to compare the feelings of the developers with the results of the tool reports. For instance, in these studies, the students believed that when they started considering technical debt, the project quality improved, but when we analyzed the code with the tools, we saw that the reports did not indicate significant changes in the code quality. Then, it is interesting to investigate why it happened, and possible future solutions (Are it because the tools identified different types of technical debts? Or because they just identified, but never paid the technical debts? Or because of the influence of others quality metrics? or Due to the team experience or because the comparison need to be done in the long-term, etc)

- Technical debt identification and monitoring: conducting a study to connect the technical debt that was contracting and the task that origin it. Thus, the teams could see what kind of tasks results in technical debt items. Then, they could organize some strategies to mitigate the occurrence of that items.

- Technical debt measuring: performing a study to test new metrics to measure technical debt, that can help teams to prioritize and organize the technical debt rank. In addition, the teams can test tools to do that, including they can develop a tool using machine learning techniques and methods. Moreover, we can improve how to measure technical debt, dealing with metrics as risk and impact on business.

- Technical debt prioritization: currently to prioritize the debts we have to consider a manual analysis and discussion of the team, we need more automated tools to support it.

- Technical debt management: the models created in this study suggested some ways to manage technical debt, it is important to test the model in other cases plus creating new templates, process, forms and combining with others tools, to generalize the model. Further, it is interesting to test new metrics and graphics to show the technical debt list. Also, it is crucial to do more analysis to understand how agile teams deal with technical debt, and whether the agile practices can influence or prevent the technical debt incurrence.

- Software quality map to provide a mapping to connect the technical debt items with the parts of the software that contains the technical debt. Also, showing which part of the business is affected by that part of the software. This research can help teams to create a technical debt rank where they can prioritize to pay that debts are affecting the most crucial part of the software.

- A comparison with the recent models for technical debt management can be done testing in software development teams. Aiming to create a unique version of the model for technical debt management.

\subsection{Chapter Summary}

This chapter summarizes the main results of this research. After, we answered each research question summarizing it according to the interpretation of the findings. Finally, we suggested some future researches in technical debt management. 
Appendix A

Paper published at XP Conference, 2017 


\title{
Effects of Technical Debt Awareness: A Classroom Study
}

\author{
Graziela Simone Tonin $\left.{ }^{1(}\right)$, Alfredo Goldman ${ }^{1}$, Carolyn Seaman $^{2}$, and Diogo Pina ${ }^{1}$ \\ ${ }^{1}$ Institute of Mathematics, Statistics and Computer Science, University of Sao Paulo, \\ São Paulo, Brazil \\ \{grazzi,gold, diogojp\}@ime.usp.br \\ 2 Department of Information Systems, University of Maryland Baltimore County, \\ Baltimore, USA \\ cseaman@umbc . edu
}

\begin{abstract}
Technical Debt is a metaphor that has, in recent years, helped developers to think about and to monitor software quality. The metaphor refers to flaws in software (usually caused by shortcuts to save time) that may affect future maintenance and evolution. We conducted an empirical study in an academic environment, with nine teams of graduate and undergraduate students during two offerings of a laboratory course on Extreme Programming (XP Lab). The teams had a comprehensive lecture about several alternative ways to identify and manage Technical Debt. We monitored the teams, performed interviews, did close observations and collected feedback. The results show that the awareness of Technical Debt influences team behavior. Team members report thinking and discussing more about software quality after becoming aware of Technical Debt in their projects.
\end{abstract}

Keywords: Technical debt - Technical debt awareness - Technical debt impact · Extreme programming

\section{Introduction}

Several studies have shown that agile methods have provided significant gains in software projects [19]. However, it is also known that when prioritizing delivery speed, as may happen in agile projects, Technical Debt may be incurred. Much of this debt is not even identified, monitored or managed. Technical Debt that is not well managed runs the risk of high maintenance costs.

The term Technical Debt was introduced by Cunningham, who explained it in the following way [4], "...Although the immature code may work fine and be completely acceptable to the customer, excess quantities will make a program unmasterable, leading to extreme specialization of programmers and finally an inflexible product. Shipping first time code is like going into debt. A little debt speeds development so long as it is paid back promptly with a rewrite [...]. The danger occurs when the debt is not repaid. Every minute spent on not-quite-right code counts as interest on that debt...". Technical Debt is recognized as a critical problem for software companies [2] and has received a lot of attention in the recent years from both practitioners and researchers $[16,17]$. 
Lim et al. [18] emphasize that: “...most project teams now recognize that Technical Debt is unavoidable and necessary within business realities. So managing Technical Debt involves finding the best compromise for the project team...", but a project team cannot do this if they are not aware of Technical Debt. Also, Lim et al. highlighted that when the development team is not aware of Technical Debt, it will probably result in challenges for maintenance and evolution tasks. Given this scenario, our motivation was to observe the effects of Technical Debt awareness in teams in an academic setting.

The Extreme Programming Laboratory (XP Lab) is a course that has Undergraduate and Graduate students at the University of São Paulo since 2001. The aim of this course is to provide the experience of a real software development scenario using the Extreme Programming values and practices [1].

Extreme Programming emphasizes teamwork; managers, customers and developers are all equal partners in a collaborative team. The main values of Extreme Programming are communication, simplicity, feedback, respect and courage [3].

The objective of our research is to characterize the impact on the team when Technical Debt items are visible, based on team members' perceptions. This study aims to answer the following research question (RQ):

\section{- What is the impact on the team when Technical Debt is explicitly considered?}

The study was applied in two editions of XP Lab. Four teams were followed in the 2013 edition and five teams in the 2014 edition. We conducted the study and collected data through questionnaires and interviews, and analyzed the source code of the projects with Sonar Qube and Code Climate tools to identify the impact on the teams that explicitly considered Technical Debt (TD).

In the next section, related work is described. In Sect. 3, we describe the context of the Extreme Programming Laboratory. After, in Sect. 4, we provide a description of the research steps, data collection and analysis. Section 5 describes the results. In Sect. 6, we discuss the findings and present the threats to validity. Finally, in Sect. 7, we present the final considerations and future work.

\section{Related Work}

Few studies deal directly with the technical debt awareness. The study of Kruchten [22] showed that agile teams believe that they are immune to TD, because they use an interactive development process. Therefore, he explains that in these teams, TD items could be contracted rapidly and massively, because code is often developed and delivered very rapidly, without time to devote to good design or to think about longer term issues. This could result in contracting TD items such as a lack of rigor or systematic tests. To deal with TD and to avoid accumulating too much TD, he suggests: "The first step is awareness: identifying debt and its causes. The next step is to manage this debt explicitly, which involves listing debt-related tasks in a common backlog during release and iteration planning, along with other "things to do.". Bavani [21] shows that if teams are unaware of the context of meaning of the term TD, they can consider trivial issues or technical tasks as a TD. These teams have to improve the awareness of it, so he proposes 
a quadrant to help teams to better recognize and understand the TD concept. The study of Martini [23] listed some causes of architecture technical debt and one of the reasons he found was the lack of awareness about the dependencies between the specific architectural TD and the other parts of the software. Furthermore, there are many related studies on not managing TD and how this affects software quality, such as in the studies of Guo [20], Sterling [24], Li [16], Lim [18], and Curtis [25]. McConnell [26] emphasizes that when a team makes the decision to contract a debt or not, they are really deciding between two ways to complete the current development task, one faster and the other resulting in better quality. Bavani [21] talks about management of TD items in distributed agile teams, and he emphasizes that the management of TD items directly affects the economics of software maintenance and according to him, the key for success in the current global economy is building and maintaining software under optimal costs. Sterling [24] said that TD exists and is detrimental to the maintenance of software quality. Buschmann [27] suggests that teams doing a refactoring in the code should also pay the TD items and improve internal quality. A recent report showed that one of the consequences of incurring TD is the impact on quality [28].

\section{Context: Extreme Programming Laboratory}

The XP Lab is a regular course offered at the University of São Paulo, to graduate and undergraduate Computer Science students. The motivation is to provide them an opportunity to learn agile software development methods on real projects. In the 2013 offering, there were four teams, with five or six students each. In the 2014 offering, five teams with six students each attended the course. XP Lab students have the support of metacoaches who are experts in agile methods. They provide agile mentoring for all the teams with the professor's help. Each team also has a coach, who is a student that has more experience in agile methods. The teams develop real projects with on-site customers. The teams have to follow some agile practices, for instance; pair programming, automated tests, continuous improvement, continuous integration, etc. In both studies, the teams worked in pairs and in threes, and the groupings changed many times during the course, sometimes according to the tasks they needed to develop. The course requires a minimum attendance of at least $8 \mathrm{~h}$ a week of dedication (four hours in the laboratory and four hours of extra classes), and there is a lunch once a week, to encourage the students' presence in the lab and to allow the students to share experiences. On some weeks, there are short presentations about some difficulties that the teams are facing, where a specialist explains and discusses specific topics. A complete description of the course settings can be found in [1].

\subsection{Projects}

In Table 1, we briefly describe each of the projects involved in our study: 
Table 1. Extreme programming projects

\begin{tabular}{|c|c|}
\hline Project & Description \\
\hline Arquigrafia & $\begin{array}{l}\text { Arquigrafia is a public digital collaborative environment, } \\
\text { nonprofit, dedicated to the dissemination of architectural images, } \\
\text { with particular attention to Brazilian architecture [6]. }\end{array}$ \\
\hline Games-VidaGeek & $\begin{array}{l}\text { A platform for games that support the teaching of programming } \\
\text { (with games for Scala, Java, Html, CSS, SQL and other languages } \\
\text { being produced) [7]. }\end{array}$ \\
\hline TikTak & $\begin{array}{l}\text { A project focused on collecting feedback data from users and } \\
\text { display it in a web dashboard [5]. }\end{array}$ \\
\hline Mezuro & A framework for monitoring source code metrics [8]. \\
\hline Monitoring system & $\begin{array}{l}\text { An online system where students can apply to be a teacher assistant } \\
\text { of a regular courses [14]. }\end{array}$ \\
\hline System specialist in sport & $\begin{array}{l}\text { An application to enable researchers working with physiological } \\
\text { data to apply metabolic mathematical models [15]. }\end{array}$ \\
\hline Social networking startups & $\begin{array}{l}\text { A social network for Startup, with the goal of creating a community } \\
\text { of highly connected and committed entrepreneurs [5]. }\end{array}$ \\
\hline Family tree & $\begin{array}{l}\text { A genealogy community where each individual can create a family } \\
\text { tree and from time to time the system attempts to "link" the trees } \\
\text { [5]. }\end{array}$ \\
\hline CoGroo & Portuguese grammar corrector used by LibreOffice [9]. \\
\hline
\end{tabular}

\subsection{The Informative Workspace}

Each team had its private informative workspace ${ }^{1}[10,11]$, where they physically displayed TD items. In the XP Lab 2013 offering, all teams had a TD board (Figs. 1 and 2). In the XP Lab 2014 offering, each team decided by themselves how to manage the TD items in their informative workspaces. Some teams decided to have the TD board and other teams kept the TD items list on a Kanban board.

\subsubsection{Boards}

In the TD board (Fig. 1) a team placed the TD items that were incurred and/or identified. On the top of the board, there is a supply of blank cards called 'Fichas'. These cards were used to document the TD items.

Figure 2 shows another team's board where they kept the list of TD items that were incurred and identified. On the right side, they have a reserve of blank cards.

\footnotetext{
${ }^{1}$ The informative workspace is the place where the teams put all the physical boards and graphics, with the metrics they used to manage the project development also the list of the task they will develop in each sprint.
} 


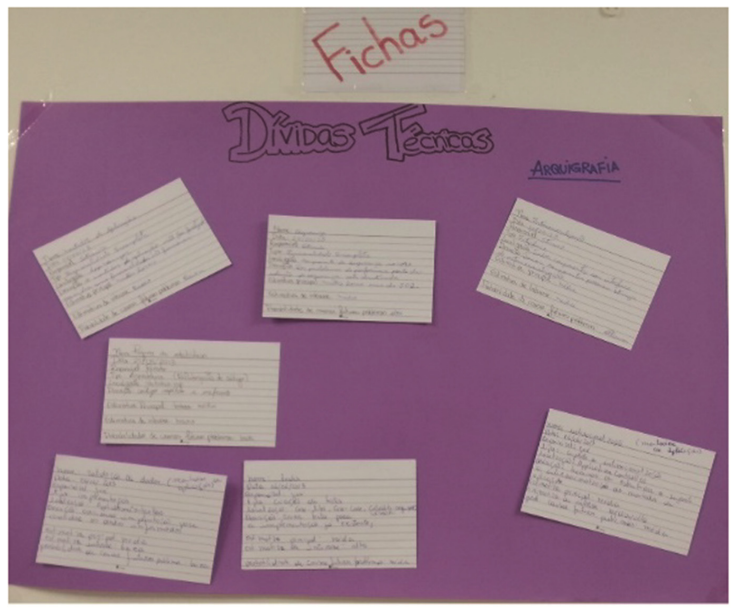

Fig. 1. Technical debt board

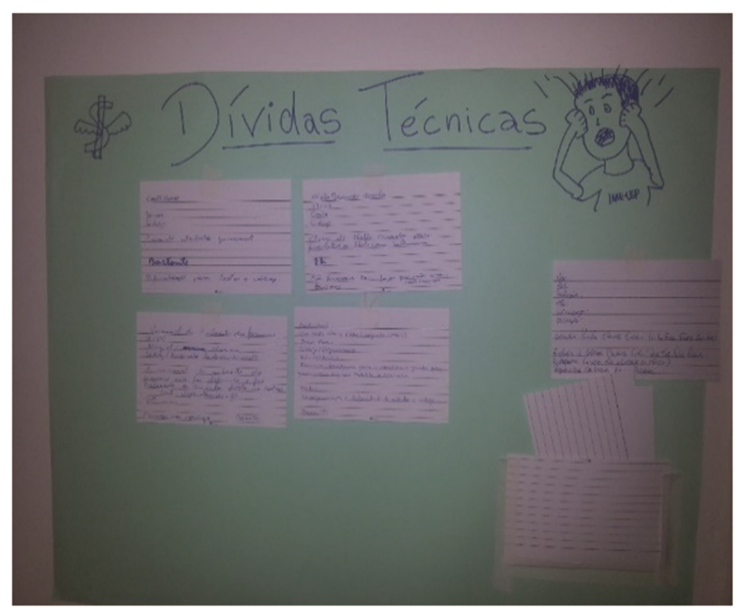

Fig. 2. Board of the technical debt list.

Figure 3 shows one TD item about duplicated code. Each card had nine categories to fill out. Below we transcribe the data contained in Fig. 3.

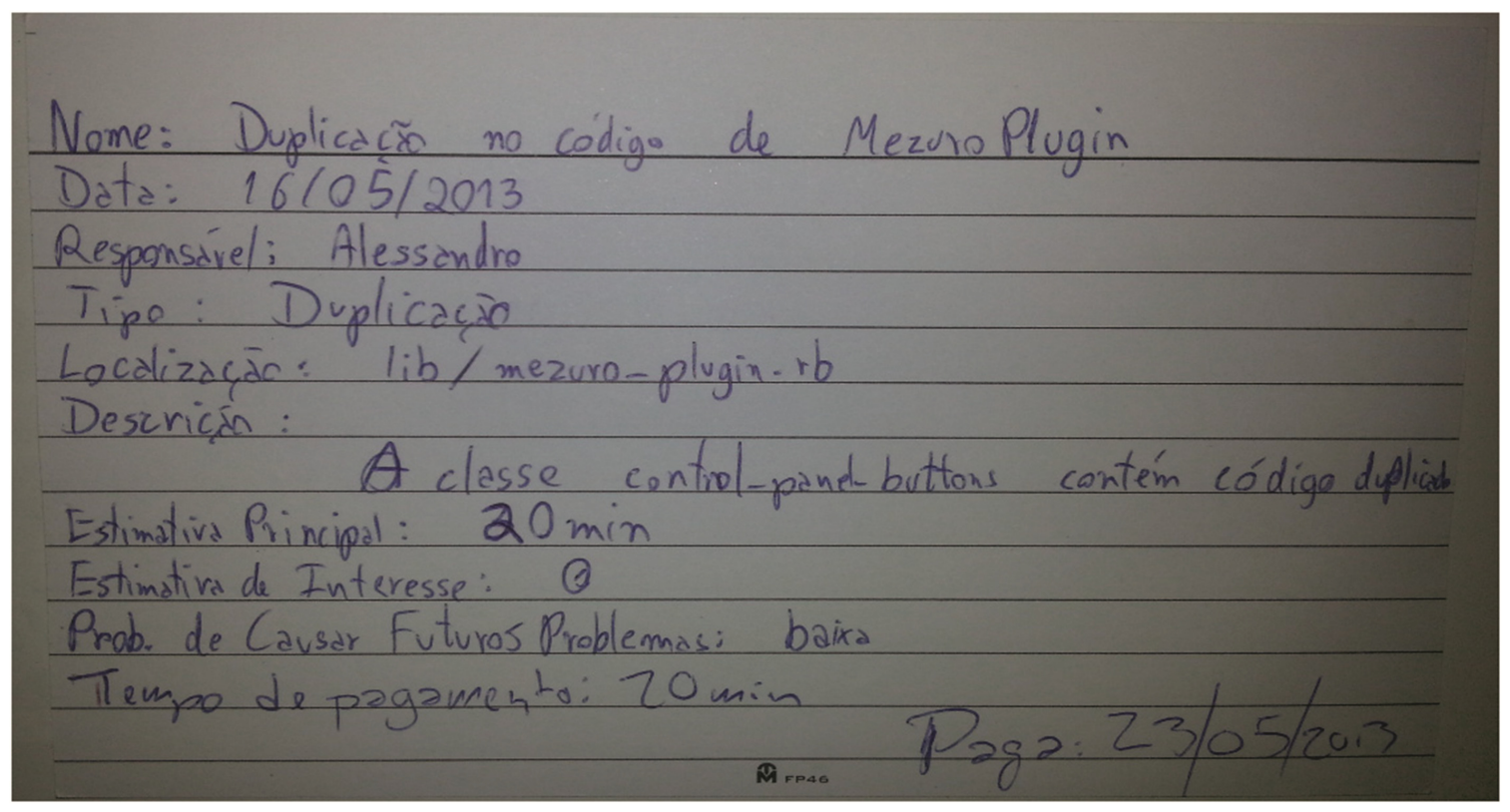

Fig. 3. Technical debt item

In this case the Name of TD item was Duplicated code in the Mezuro plugin, the Date (when the item was identified), was 05/16/2013, the Responsible (the person that incurred or found the TD item, in this case Alessandro), the Type was duplication, (could be test, documentation, design, etc.), the Location (which part of the code the items was related), was in lib/mezuro-puglin-rb. The Description (a brief description of the TD item), the class control-panel-buttons has duplicated code. The Estimated Principal, was twenty minutes (how much expected time they need to spend now if they implemented that task in the correct way, if they did not know how much time, then they could use a scale of high - if they probably will spend a large amount of time -, medium - if they probably will not spend much time - and low - if they probably will solve it quickly). 
The Estimated Interest Amount should be filled out when they pay the TD item. The Probability of being a future problem (i.e. the interest probability) in this case was low. In this case, they also added in the card the Date when they paid the TD item, 05/23/2013 and how long it took them to pay off the item, also twenty minutes.

These boards represent some of the boards used in the team's informative workspace. Some teams used a specific board to manage TD, as shown in Figs. 1 and 2, other teams used the Kanban board and put the TD items together with the tasks of the sprint. Furthermore, some teams also placed the list of TD items in the tool used to manage the project.

\subsubsection{Tools}

The teams had two code quality analysis tools:

- Code Climate: a tool for quality analysis of code repositories (https://codeclimate.com/).

- Sonar Qube: a code quality analysis platform that has a plugin that identifies TD (http://www.sonarqube.org/).

\section{Research Methods}

Data was collected and analyzed for this study through interviews and questionnaires. Before data collection, the teams spent some time identifying TD items in their projects. Below, we first describe how TD items were identified, and then we describe our data collection and analysis methods.

\subsection{Technical Debt Identification}

In the two offerings of the XP Lab, we followed slightly different steps to help the teams to identify TD.

\subsubsection{XP Lab 2013}

Four weeks after the students started working on their projects, we gave a presentation about TD, as some students were familiar with the term, but others were not. After the presentation, we had a discussion and we encouraged the students to talk about their views of TD. We also talked about some concrete examples they had in their projects. The discussion lasted for about one hour and after that, each team had to prepare a TD board, where they would have to document their TD items (Fig. 3 shows an example of a TD item). Each TD item was written on a card, and in this card they had to fill out a list of topics, after that it was then pinned to the board. The card structure was based on the template developed in [12]. Each team had a board with a set of cards representing TD items. Every team that identified or incurred a debt put the information on the board. 


\subsubsection{XP Lab 2014}

In the XP Lab 2014 offering, we made a presentation about TD for all the students together for $30 \mathrm{~min}$, about two months after the course began. After that, we discussed it for another $20 \mathrm{~min}$, during this time the students could clarify their doubts about TD. The students already had the code quality analysis tools available, Sonar Qube and Code Climate, since the beginning of the course. We did not impose the use of either the boards or the tools to identify TD. We showed them the meanings of TD items and some examples in each project. We also presented examples of a TD board and of the categories they could use to identify TD items. Then each team decided whether they would monitor TD on their project or not.

\subsection{Second Step - Interviews and Questionnaires}

Data collection was done differently in the two XP Lab offerings. In both cases, similar data was collected both at the beginning and at the end of the course.

\subsubsection{XP Lab 2013}

Eight weeks after the teams started to identify TD items and fill the boards with cards, we carried out a face-to-face interview with the pairs in each team. The interview motivation was to verify the influence on the team of the TD visibility. The interview was composed of twenty questions, with open-ended and multiple-choice questions, separated into the following topics ${ }^{2}$ :

- The concept of TD.

- Were there any changes in the software development process?

- Negotiation with clients.

- About the experience of identifying TD.

- What is the relevance of identifying TD?

- What is the impact on software quality?

- Do the teams pay off TD?

- Will the teams pay off some TD?

Four weeks after the first interview, at the end of the course, we did the last interview with an open format and we performed it for each team. In the last interview, each team was invited to talk about the experience of making TD explicit. Each interview took about twenty minutes.

\subsubsection{XP Lab 2014}

In this edition of the course, we decided to apply a questionnaire on what each team member thinks about TD (the questionnaire was answered by the students individually; this approach was taken to try decrease a possible bias). This questionnaire was applied

\footnotetext{
2 It is possible to access all the questions in the following link https://www.dropbox.com/sh/ gen3dr97xxofs21/AACo11oqbBsaCprOCQtSYv5Ja?dl=0.
} 
one week before the class received a talk about TD. We sent a link to the questionnaire by email and then the students had one week to answer it.

The questionnaire was composed of seventeen open-ended and multiple-choice questions, separated into the following topics ${ }^{3}$ :

- Software quality

- What does the team do about quality?

- Familiarity with TD.

- Do you know about and use the TD concept?

- How TD is used in the project.

- Are you using the Sonar Qube or Code Climate report?

- Are you using a TD list?

- Have you paid any TD item?

- Is there any evidence that having the TD items visible has an influence on the team?

- Did you identify any TD item that was not identified by the tools?

- Are you going to consider TD in future projects?

- Do you think the TD concept is relevant?

The same questionnaire was applied a second time at the end of the course. The aim was to see if there was any change in the team members' behavior.

\subsection{Third Step - Data Analysis}

For the data analysis, we used coding techniques from the grounded theory approach [13]. Grounded theory methods are aimed at building or discovering a theory. In this approach, the data analysis proceeds in three interdependent steps: open coding, axial coding, and selective coding. In the open coding step, the researcher interprets the data to identify patterns and define codes, “...event/action/interaction, are compared against others for similarities and differences; they are also conceptually labeled [...] conceptually similar ones are grouped together to form categories and their subcategories..." [13]. In axial coding “...categories are related to their subcategories, and these relationships tested against the data..." [13]. Then in selective coding "...all categories are unified around a central 'core' category and categories that need further explanation are filled-in with descriptive details..." [13].

For data analysis, we used the $\mathrm{NVivo}^{4}$ tool, which is widely used for analysis of qualitative data. In this case, the goal was not to use grounded theory to develop a new theory but only use its coding steps to answer our research questions.

We also analyzed the source code of the projects with the Sonar Qube and Code Climate tools, to try to identify relationships between team's beliefs and the reports from these tools.

\footnotetext{
${ }^{3}$ It is possible to access all the questions in the following link https://www.dropbox.com/sh/ gen3dr97xxofs21/AACo11oqbBsaCprOCQtSYv5Ja?dl=0.

$4 \mathrm{http}: / /$ www.qsinternational.com/support/downloads/nvivo-9.
} 


\section{Results}

In this section, we describe our findings organized by the coding steps. As the main question in the both editions of XP Lab was the same and the obtained results were similar, we analyzed the results of both editions together.

\subsection{Open Coding}

In this step, the data analysis was conducted by reading the transcripts of the interviews and also the answers from the questionnaires. We applied the coding process to this material, line by line. In this phase, we discovered the open codes. In Table 2, we list three code samples. It is possible to access the list of the open codes that emerged from this first codification in an appendix ${ }^{5}$.

Table 2. Example of codes resulting from open coding

\begin{tabular}{l|l}
\hline Open codes & What they talked about \\
$\begin{array}{l}\text { Changed attitude of the } \\
\text { teams }\end{array}$ & $\begin{array}{l}\text { The team discussed more the tasks they have to do before incurring } \\
\text { a TD and they thought more before taking the decisions. }\end{array}$ \\
\hline Communication & After the identification of TD items, the team had more discussions. \\
\hline Maintainability & $\begin{array}{l}\text { The identification of TD items helps the teams to know that there will } \\
\text { be some changes in the software in the future. }\end{array}$ \\
\hline
\end{tabular}

\subsection{Axial Coding}

The open codes were reassembled in new ways during axial coding to form categories. The goal was to create a higher abstraction level. Thus, codes were grouped to form subcategories, and in turn, they were organized into categories. This process was highly iterative, with codes and categories forming and re-forming as more data were incorporated into the evolving understanding [13].

In Fig. 4, it is possible to observe the list of categories and subcategories resulting from axial coding analysis. The first level is the main category resulting, this category emerged from the subcategories of the second level, the subcategories are resulting from the codes emerged in the third and fourth level.

One of the most important influences when we make a list of TD items is the attitude of the team (team behavior), "...registers by not forgetting, there was a change in the attitude of the team..." and "...increased people's concern regarding the Technical Debt...". The team had less 'untouchable' expert professionals and behaved more as a whole team. They talked more about the TDs “...we discussed these debts. Otherwise, the project would not have advanced..." and thought about the necessity of incurring it. It helped them to have the same understanding of the concept of TD because they discuss it (TD concept). In addition, if the team members were not sure whether to incur

\footnotetext{
${ }^{5}$ It is possible to access all the codes in the following link https://www.dropbox.com/sh/ gen3dr97xxofs21/AACo11oqbBsaCprOCQtSYv5Ja?dl=0.
} 


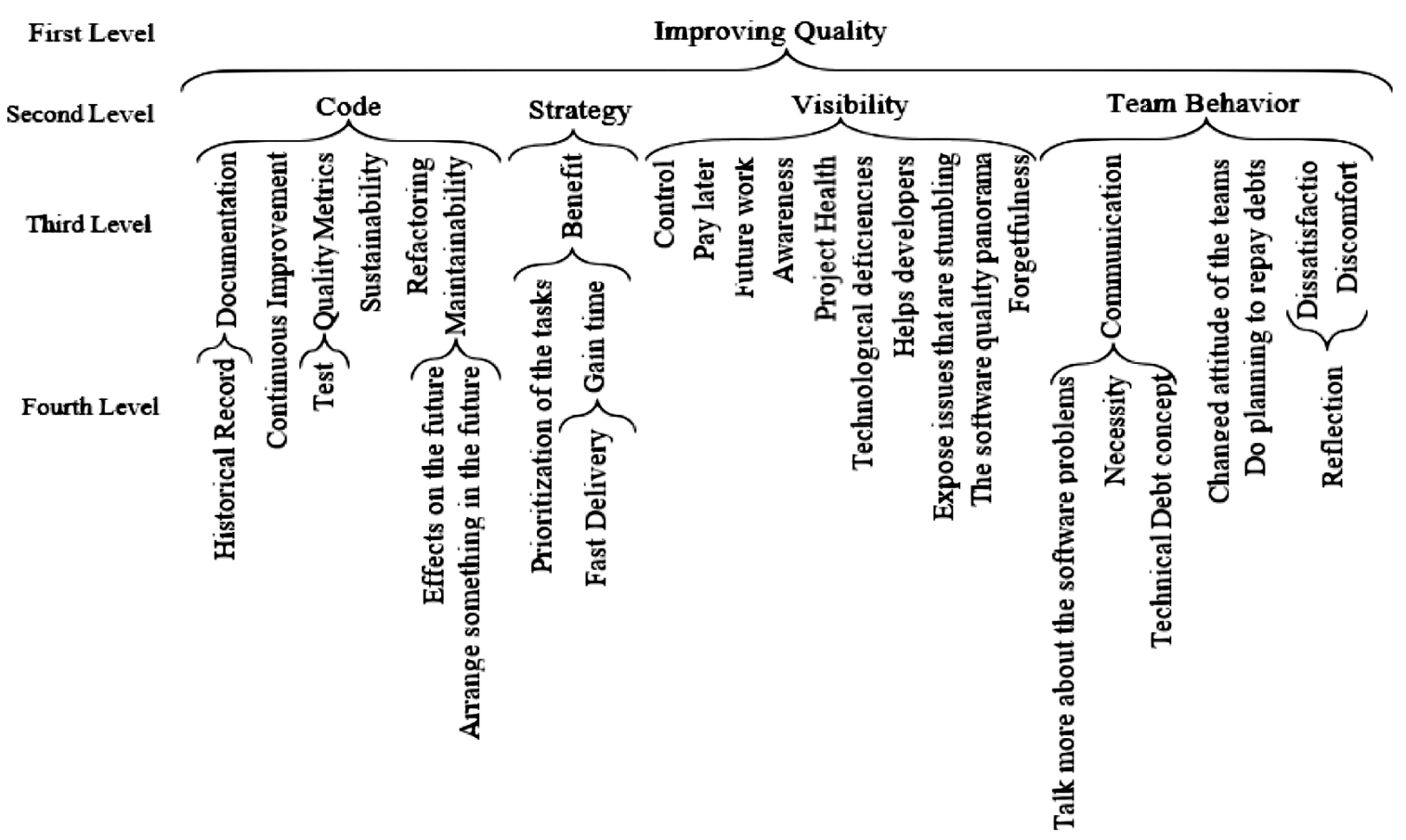

Fig. 4. Categories and subcategories of the XP Lab 2013 and 2014 edition

a TD item or not, the team member debated with another team member to help him to take this decision, thus improving the team's communication. Team members started talking more with each other and because of it, they knew what part of the project was being modified and the problems of the software, "... It was easier to remember that we have to fix things, debts...". Furthermore, if team communication was good, they were more comfortable to share with each other their difficulties. After the team began to identify TD, developers discussed their decisions rather than just doing something and moving on, now that all the software problems were more clear to the team members, "...usually only the person thought or knew about it (...), now with the TD it becomes clearer as well...". They began to argue among themselves, before incurring a debt, “... As evidenced here we even got everyone talking about the debts, instead of just looking to give a quick solution and move along...".

They started to think more about if it was necessary or not to incur some TDs. Several times they concluded that it was not required, a team member says: "[...] From the moment I started to think about that item, which was debt, I asked myself about what is the current cost, compared to the future cost. Because if the cost of doing now is less, then it's better to fix it now...", other student says: “...we think twice before making a TD...".

When TD items were visible, the team had more control, over whether they will pay off the TD item, whether they will incur more debts or not, or whether they will incur and pay later. Moreover, this facilitates planning to repay the TD items, “... we analyzed some of the debts, now we will plan, what we might kill, to kill some of those debts, then it becomes easier to make this analysis...". Therefore, if they incur a TD item, they would make it visible, would monitor it, and sometimes they would look back at this TD item. This way, they always thought about continuous improvement. Thus, the team could be defining a strategy to pay off or not some TD items to improve the code quality. 
However, if the team member incurred a TD item, and never paid, the project would probably lose quality. Nevertheless, they might incur TD to prioritize other tasks or as a business decision to deliver fast and then used it strategically.

The TD item list presented indications on whether the code quality was improving or not, and helped them to understand the current development state It also indicated if they would have a lot of future work and future refactoring to do in that code. Indeed, if the software had TD items, the team would probably need to perform some refactoring, and it directly affected the sustainability of the project. Furthermore, the team could do an analysis of the TD items and they could be defining a software's quality metrics to help them monitor the software quality, for instance, test. They also could identify technological deficiencies and define possible directions to improve their technical skills and not repeat the same mistakes, in order to mitigate the occurrence of those TD items that were recurrent.

The teams used the TD item list as documentation providing a historical record of the immature parts of the project. Therefore, each team knew that some parts of the project should be improved; it was possible to see if there was a TD item to pay off. This documentation helped the teams to maintain the code and it reflected on the health of the project. In addition, it affected the teams by sometimes causing dissatisfaction with the quality of the software. Many team members saw that it was very uncomfortable to arrive at work and see that the health of the project was not so good. If the source code health of other teams was good, it was even more uncomfortable. Therefore, this process of the team having discussions, and having dissatisfaction impacted in the following reflection: when a team member decided to incur or not $\mathrm{TD}$, thought and discussed it, he better understood the problem that he had to solve. This often resulted in the noninsertion of a TD item in the code, since it only lacked the understanding of what should be done.

Considering TD implied generating a culture focused on quality. It affected factors related to project continuity. It has an influence on the cost and the viability of maintainability and evolution of the project. A developer said that, if they did not have the TD items visible it was so difficult to identify the software quality landscape, "... it was difficult for you to identify the whole landscape...". Therefore, if in the future the team needed to make some changes in the legacy code, they already knew what they were and where the problems were located. It provided a general awareness to the team about the problems of the software. It also might help a new team member that did not know about the code to have a notion on the quality of the code and the location of the code problems.

\subsection{Selective Coding}

Selective coding constituted the third stage of data analysis, with the objective to refine and integrate categories, unveiling a category deemed as central, encompassing all the others. The full potential of abstraction was employed to incorporate the full scope of the data investigated and coded [13].

In our case, the objective was not to generate a theory, but rather to identify the main categories. The aim was to describe the impact of TD awareness. 
The categories, Strategy, Team Behavior, Code, and Visibility represent the main influences on teams due to making TD items explicit. Each of these categories captures part of our results, although none of them describes the phenomenon entirely. For this reason, another abstract category is required, a conceptual idea on which all categories are included. As such, we concluded that the resulting core category might be a perceived notion on "Improving Quality".

All teams progressed towards creating a culture of Quality of the code, team, and project. This arose primarily because each team member started to think more about the need to incur TD items. Many times they decided that incurring TD was not necessary in a given situation. When a team member was not sure about the necessity or not to incur the debt, they spoke with other members to make a decision. Therefore, the team improved their communication and then it was clearer what each member was doing. So, it was easier to understand the objective of the project. Then, making TD explicit has a direct implication on the Team Behavior. Most of the team members said that after they started to identify TD, they talked more with each other, thought more about the real need to incur debt or not, discussed more about code quality, refactored more frequently some parts of the code, knew where the code problems were, and where each team member was working at any time on the project. In addition, when communication among the team was good, people felt more comfortable to expose and discuss their problems with the team. The team then became more a group that works together, rather than a group of experts on different parts of the system.

In some situations incurring some TD items was a Strategy to gain some time, due to the time to market. Moreover, if there was a list of TD items it might be possible in the future to correct them, by refactoring. The list of TD items was used as documentation, this enabled the historical record of the TD items list that the code contains. When the need to change a particular part of the system appeared, it was possible to verify if it had some TD item and if this debt would affect such functionality. Therefore, the team members had the option of paying it off or not. The documentation helped in the Visibility of the project's health. If the software had any TD, probably it would have more deficiencies.

Finally, when a team had the TD items list they automatically became Aware of the TDs of the project, consequently about the software quality. So, the team could think about it, before possibly incurring in another TD item. The team could decide when and where they would improve the software quality.

\subsection{The Code Analysis with the Tools}

In XP Lab 2013 edition, we analyzed one project with Code Climate tool (supported the language used in the project, in this case, Ruby). Further, we analyzed two projects with the Sonar Qube tool. In the XP Lab 2014 edition, we also analyzed two projects with Code Climate tool and four projects with the Sonar Qube. We considered the Code Climate metric, grade point average ${ }^{6}$ (GPA) and in the Sonar Qube the following metrics: code smells, security, reliability, maintainability, duplications, documentation,

\footnotetext{
${ }^{6} \mathrm{https}: / /$ docs.codeclimate.com/docs/code-climate-glossary\#gpa.
} 
issues, technical debt rating, complexity, size, duplicated blocks, bugs \& vulnerability and duplications. The GPA of the Mezuro's code and the Monitoria project increase after the teams considered TD. Then the quality of project increased, it means that the remediation (the amount of effort required to improve a software issue), was 0 to $2 \mathrm{M}$ (too short). In the analysis with Sonar Qube of the projects: Game VidaGeek, Tiktak, Arquigrafia, Family Tree, Social Networking Startups and Specialist in Sport we did not identify large variations in the measured metrics comparing the two different versions of each project (Before and after they consider TD). However, in 5 of the 6 projects, the rate of duplication and the duplicated blocks decreased after the team started to consider TD, indicating an improvement in code quality.

It is important to highlight that the students said that most of TD items were not identified by the tools, which explains the modest size of the changes in these metrics. For instance, one team was using Handsontable ${ }^{7}$ for data entry, and they had a problem with the validation of the data. The presence of this type of TD item, nor the impact of paying it off, could not be identified with Sonar Qube or Code Climate reports. In general, many types of TD items (e.g. those related business rules) cannot be identified through static metrics.

\section{Discussion of the Findings}

The teams had some similar views on the importance and benefits of making TD explicit. A significant finding is that the teams considered it very helpful because they could see the whole landscape of the software quality (they knew which part of the software had immature code). They also emphasized that it was very useful to have a board where every day they could see the health of the code. Before becoming aware of TD, the team members reported that they sometimes incurred TD but never remembered to go back and correct it. But after considering TD, they thought about the necessity of incurring TD and often decided against it. Also, they could see the TD list and so they did not forget the TD items that needed to be addressed. They discussed more about how to implement the tasks, also they talked more about the problems of the software because they had the list of the TDs visible. This process of thinking about incurring or not TD, discussing about it and reviewing the TD during the project can create a culture focused on improving the software quality.

In addition, in this study we explored some ways of identifying and monitoring TD. Our subjects found some form of a TD board very useful for documenting TD, making it visible, and adjusting both the TD board and their behavior accordingly. By using the TD board, they always know the list of software deficiencies so have a constant reminder of how to organize their work and improve the software. As a complementary aid, they may use tools to help them to identify and monitor TD occurrence. However, it is important to highlight that tool reports provide a static analysis of the software quality and some TD item could not be identified using static metrics.

\footnotetext{
${ }^{7}$ https://handsontable.com/.
} 
The results of this study could motivate teams to consider TD further, to help developers convince leaders and directors, the decision makers, to start considering TD. These approaches used by the XP Lab teams, such as boards, cards and tools can help teams in companies to deal with TD. In addition, they could define the list of TD items that are crucial to the project but hard to identify with the tools. As a result they can define a strategy to deal with the TD over time.

\subsection{Threats to the Validity}

In this study, we took some actions to mitigate possible biases, we describe these in the following points:

- Construct validity (credibility): We used multiple data collection approaches with the aim to reduce possible bias. When planning the interviews and the questionnaire we discussed the best way to formulate the questions. We did a first interview and questionnaire with one member of the group as a pilot test. Based on this test we reviewed the questions. We did not include these data in the final analysis. One thing that it is important to highlight is that the students might not have understood the main meaning of the questions correctly, in the interviews and questionnaires. Because of this, in the interviews, if the student did not understand the question the interviewer explained the question for them. The researcher was available throughout both studies if the students had any questions.

- External Validity: This study can be replicated in other academic courses, also in companies. In both cases, the study can be separated into two parts and can be applied in these situations: one in teams that do not consider technical debt yet, to verify if awareness of TD influences something in the team, such as communication. Furthermore, this study can be analyzed with teams that already consider technical debt, by identifying, monitoring and managing if it is possible to identify some changes in the team behavior and in the software quality. If they have a historical record, we could also measure the software quality with tools. Finally, to carry out this study is not necessary to make significant changes in the team's environment, which makes feasible to replicate in companies.

- Internal Validity: We analyzed the data separately, first the interview transcriptions, then the questionnaire responses, and then compared and merged the findings that were relevant and had a lot of evidence in the results of both studies. In the case of any doubt about a specific point, we went back to the data and re-analyzed them. After that, the advisor and co-advisor read the results and if they indicated some points to be re-analyzed, the researcher re-analyzed the data. We did analysis and reanalysis many times until we were sure of the conclusions.

- Reliability: To interpret the data we followed the coding techniques from the grounded theory steps. Also, the data analysis was made by a single researcher, however, the results of the analysis were discussed by the two researchers and with the advisor and co-advisor, every time a doubt arose the data were re-analyzed. Also, this paper is a result of an analysis of the data that lasted two years, where the researcher compared the data many times. Furthermore, the preliminary results were 
presented and discussed at seminar ${ }^{8}$ attended by top researchers in this field. It is important to observe that when we infer that awareness of TD could impact software quality, we are describing the perceived quality by the team.

- Objectivity: The results show the information derived from the data, the codes and categories emerged were related with the data quotes.

\section{Final Considerations and Future Works}

This work describes results about the influences of making TD explicit in an academic setting. Our results show the importance of making TD visible and how that influences teams. It is important to point out that no negative influences were identified. The team members were always very excited about the results of making TD items visible. As communication in the team was improved, all team members thought more about quality, not just specific members. The "agile" culture of the teams improved and in addition, the team believed that it was easier to show the impact of the TD level to clients, showing that it is possible to invest some time to improve the quality. The main results emphasize the Extreme Programming values and helped the teams to support values such as communication at all levels, courage to change and feedback to continuously improve the software.

In future work it is important to verify the influence on the team in the long term, especially concerning speed and code quality. It is also important to create ways to compare the perceptions of the developers with the results of the tool reports. For instance, in these studies the students believed that when they started considering TD, the project quality improved, but when we analyzed the code with the tools, we saw that the reports did not indicate significant changes in source code quality. Then, it is interesting to investigate why this happened, and possible future solutions.

Acknowledgments. We are grateful to all the students and TAs of the XP Lab course (2013 and 2014 offerings) for providing valuable data for this research. We would like to thank IBM, CNPQ, CAPES and FAPESP, too, for funding this work.

\section{References}

1. Santos, V. et al.: Uncovering steady advances for an extreme programming course. CLEI Electron. J. 15(1) (2012). paper 1

2. Edith, T., Aybuke, A., Richard, V.: An exploration of technical debt. J. Syst. Softw. 86, 1498$1516(2013)$

3. Kent, B.: Extreme Programming Explained: Embrace Change. Person Education Inc, United States (2005)

4. Ward, C.: The WyCash portfolio management system. In: Addendum to the Proceedings on Object-Oriented Programming Systems, Languages, and Applications, pp. 29-30 (1992)

5. Extreme Programming Projects - CCSL. (http://www.ccsl.org.br/oldwiki/index.php)

6. Arquigrafia. http://www.arquigrafia.org.br/. Accessed May 2016

${ }^{8}$ http://www.dagstuhl.de/de/programm/kalender/semhp/?semnr=16162. 
7. VidaGeek. http://aprenda.vidageek.net/. Accessed May 2016

8. Mezuro. http://mezuro.org/pt. Accessed May 2016

9. CoGroo. http://ccsl.ime.usp.br/cogroo/. Accessed May 2016

10. Oliveira, R.M., Goldman, A., Mello, C.: Designing and managing agile informative workspaces: discovering and exploring patterns. In: Proceedings of the 46th Hawaii International Conference on System Sciences (2013)

11. Oliveira, R., Goldman, A.: How to build an Informative workspace? an experience using data collection and feedback. In: Agile Conference (2011)

12. Seaman, C.: Technical Deb Minicourse. At the University of São Paulo (2013)

13. Corbin, J., Strauss, A.: Grounded theory research: procedures, canons and evaluative criteria. Zeitschrift fur Soziologie 19(6), 418-427 (1990)

14. Monitoria. www.monitoria.ime.usp.br. Accessed May 2016

15. System Specialist in Sport. http://journals.plos.org/plosone/article?id=10.1371/journal.pone. 0145733. Accessed May 2016

16. Li, Z.G., Avgeriou, P., Liang, P.: A systematic mapping study on technical debt and its management. J. Syst. Softw. 101, 193-220 (2014)

17. Poliakov, D.: A systematic mapping study on technical debt definition. Lappeenranta University of Technology School of Industrial Engineering and Management Degree Program in Computer Science (2015)

18. Lim, E., Taksande, N., Seaman, C.: A balancing act: what software practitioners have to say about technical debt. IEEE Comput. Soc. Softw. 29(6), 22-27 (2012)

19. VersionOne.: State of Agile Report (2015). http://info.versionone.com/state-of-agile-reportthank-you.html

20. Guo, Y., Seaman, C.: A portfolio approach to technical debt management. In: Proceeding of the 2nd Workshop on Managing Technical Debt (2011)

21. Bavani, R.: Distributed agile, agile testing, and technical debt. IEEE Softw. 29, 28-33 (2012)

22. Kruchten, P., Nord, R.L., Ozkaya, I.: Technical debt: from metaphor to theory and practice. IEEE Softw. 29, 18-21 (2012)

23. Martini, A., Bosch, J., Chaudron, M.: Architecture technical debt: understanding causes and a qualitative model. In: Proceedings of the 40th Euromicro Conference on Software Engineering and Advanced Applications (2014)

24. Sterling, C.: Managing Software Debt: Building for Inevitable Change. Addison-Wesley Professional, Boston (2010)

25. Curtis, B., Sappidi, J., Szynkarsky, A.: Estimating the size, cost, and types of technical debt. In: Proceedings of the IEEE 3rd International Workshop on Managing Technical Debt (MTD 2012) (2012)

26. McConnel, S.: Managing Technical Debt. http://www.construx.com/File.ashx?cid=2797. Accessed April 2008

27. Buschmann, F.: To pay or not to pay technical debt. IEEE Softw. 28(6), 29-31 (2011)

28. Avgeriou, P., Kruchten, P., Ozkaya, I., Seaman, C.: Managing technical debt in software engineering. Dagstuhl Rep. 6, 110-138 (2016) 
Appendix B

Paper published at Brazilian Workshop of Agile Methods, 2014 


\title{
The effects of a model for identifying and managing Technical Debt in Scrum project
}

\author{
Rogerio C. Pires ${ }^{1}$, Graziela Tonin ${ }^{2}$, Alfredo Goldman², Renan Oliveira ${ }^{2}$ \\ ${ }^{1}$ Technological Research Institute of the State of São Paulo (IPT) \\ ${ }^{2}$ Instititute of Mathematics, Statistics and Computer Science - University of São Paulo \\ (USP) \\ Rua do Matão, 1010 Cidade Universitária - São Paulo - SP - Brazil \\ \{grazzi, gold\}@ime.usp.br, \{rogerio.chaves, renandemelo\}@gmail.com
}

\begin{abstract}
Technical Debt is a metaphor in the software engineering process [Cunningham 1992] that compares the negligence of quality to as acquiring a debt. In this context, we performed a multiple case study conducted on three software projects in a large Internet Company trying address the following question: "How to effectively manage Technical Debt in an agile project?". We conducted the study by proposing a model for managing Technical Debt and applying a qualitative survey to collect team member's perceptions - further coded and analyzed. As an outcome of this study, a model for managing Technical Debt in agile teams emerged from the experience, which we provide for agile practitioners interested on additional guidance in this topic.
\end{abstract}

\section{Introduction}

Events in which quality in software engineering is neglected often occur in an ordinary software development project. Such events might happen for several reasons, such as a tight budget, lack of time and/or resources, and the need to deliver features rapidly in order to maximize the return of the investment (Return of Investment - ROI). In this sort of scenario, immature artifacts are often inserted into the software developed by team members. This phenomena is informally known by software practitioners as Technical Debt - a metaphor presented for the first time by Ward Cunningham [Cunningham 1992] while describing his experience in the conference Object-Oriented Programming, Systems, Languages \& Applications (OOPSLA) 1992:

"...Shipping first time code is like going into debt. A little debt speeds development so long as it is paid back promptly with a rewrite... The danger occurs when the debt is not repaid. Every minute spent on not-quite-right code counts as interest on that debt. Entire engineering organizations can be brought to a stand-still under the debt load of an unconsolidated implementation, object-oriented or otherwise..."

The metaphor of Technical Debt is an analogy for financial debt. It claims that when the quality of the software is compromised in detriment of any other benefit, it is the same as if you were in debt. Interests are added to the debt in a way of implementing future changes in the system. If the debt is not controlled, part of future development of the team might be compromised in paying interests. 
Recently, the concept of Technical Debt has gained popularity among both in the industry and the academia. Sometimes, due to a strategic decision during the process of development of a system, the quality of the code is mainly compromised given constraints imposed on the process such as restrictions of time or other resources. Moreover, developers usually have the option to compromise the quality of the software in order to meet urgent demands in another dimension of the system - time to market, for example. Such an event is sometimes compared to acquiring a debt that needs to be paid in the future. In this analogy, this event implies on interests that can go up in terms of time, efforts or extra costs of future changes [Guo 2009].

Technical Debt has been a topic of many discussions in blogs, forums, lectures and events. On the one hand, there are many professionals in the field of the development trying to figure an effective way out to apply this concept in their companies. The academia is also interested in the topic. Some research has been done in the area intending to formalize a theory on Technical Debt [Brown et al 2010] while others aim to discover helpful ways to identify and manage it [Guo 2009] [Guo and Seaman 2011] [Guo at all 2012] [Tonin 2011]. However, there is still a paucity of empirical evidence on how to use, effectively, the concept of Technical Debt, while it has been responsible for great losses in the economy. For example, a recent study points out that approximately U\$500 billion were spent in 2010 with Technical Debt and it is estimated that until 2015 the expenses will reach US\$1 trillion [Gartner 2012].

In turn, some works in the software community report that companies have adopted Agile Methods as a way to manage software development in the last ten years. The benefits of the agile development paradigm have been proved meaningful, while there is some evidence of improvements in important areas such as productivity, the stress and customer satisfaction [VersionOne 2011] [Williams 2010]. Research have pointed out that agility is a valuable paradigm for organizational success, while studies from MIT show that Agile companies increase the income $30 \%$ faster and generate $37 \%$ more profit than the NonAgile [Highsmith 2011]. However, according to Highsmith [2011], quality, or the lack of it, is still the main point for the effective agile.

In this paper, we describe a study applied in a few agile projects in a large Brazilian Internet company aiming to explore the possibilities for managing Technical Debt in an agile environment. It is organized as follows. In Section 2, we describe the design of our research. In Section 3 we describe the data collected during the study, while we report our results in Section 4 and present our conclusions in Section 5. Additionally, we describe threats to the validity of this study in Section 6 and work to be further addressed in this topic in Section 7.

\section{Research Design}

Given the paucity of empirical studies addressing the topic of managing Technical Debt, specifically in agile software projects, we performed a study in order to better understand the effects of applying a Technical Debt management model for agile teams. After performing an initial literature review, we could not find any empirical study addressing the following research question, which we propose to help in answering:

RQ. How to effectively manage Technical Debt in an agile project? 


\subsection{Research Method}

Given the contextual nature of both topics of agile development and Technical Debt management, we decided to address the research question by performing a multiple case study. As described by Yin, case study research is known by allowing the investigation of "a contemporary phenomenon within its real-life context, especially when the boundaries between phenomenon and context are not clearly evident" [Yin, 1989].

In this multiple case study, we selected three different agile projects in the same department of a Internet Software Engineering company in order to apply the same research design protocol. We followed the same steps for all projects in order to deeply understand their specific contextual characteristics and how they affect management of Technical Debt. Another advantage of multiple case studies is providing to the researcher a higher level of generality on the study results. Moreover, both similarities and differences of each case become evident as one compares collected data from different cases.

We divided the research protocol performed in each case as a series of steps, which included acknowledging each project context characteristics, unifying the concept of Technical Debt among the team members, identifying specificities each process by participating in some traditional ceremonies in agile development, and proposing a framework for managing Technical Debt for each environment. At end, we collected qualitative data throughout a survey applied to all team members. The overall research design is available in Figure 1 and is described in the following subsections.

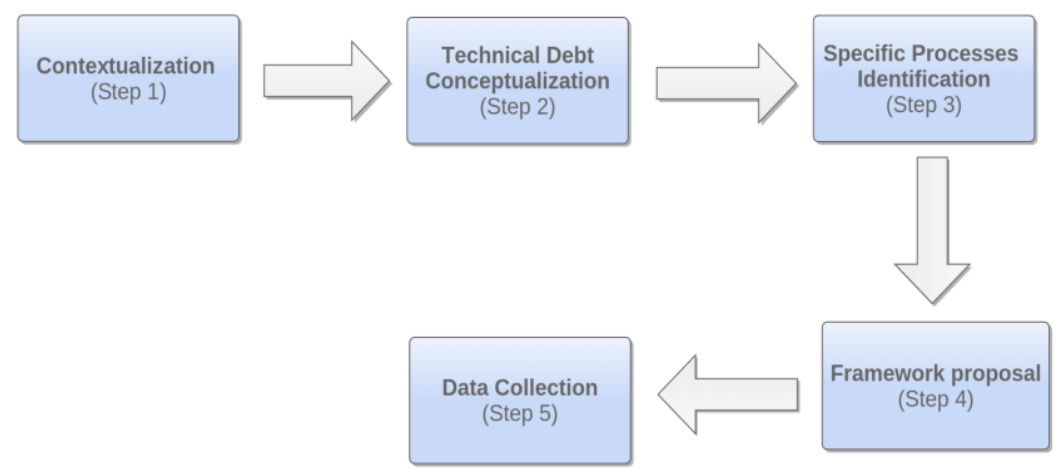

Figure 1. Research Design

\subsection{Context}

This research was carried in a large IT company with many products focused in the Internet industry. The company was founded in 1996, originally as an Internet service provider focused in dialed service content. Thereafter, it started to diversify its range of products and services while began to evolve its technologies and practices usually adopted in the Internet industry. The company software engineering staff is currently composed by more than 150 developers, divided into approximately 30 teams. We conducted the study on three selected projects from the same department - internally called as Research and Development (R\&D) - which is responsible for the conception, development, and maintenance of several software products and services provided by the company's Internet portal. All projects were performed by software teams that claimed to base their development practices on agile development - having a higher focus on Scrum. 


\section{a) Monitoring and Automation System project}

This project has started in 2008 , with the purpose of creating a product to monitor and automatize the products developed by the company. It works as an integrated platform of all company products. The product development tools are basically Java, Perl, Puppet, Tomcat, Webservice Jet, System of Queue control ActiveMQueue and Nagios. They informed that it is not a system, but a software platform that could be integrated in the all company systems.

b) Host platform project

The project has also started in 2008. In the occasion, a plan of the system was done. The system should be developed in three or four months, due to the company strategies in launching in the market of host sales. The current project technical leader begun in the company in 2010 , and has taken a product in production with the modules in the initial version, totally developed and based on a monolithic system with a large technical dependence of other systems. The development process, until 2010, was not integrated to the process based on the Scrum defined by the company. Because of this, they followed the development without adopting the patterns and process existing so far. The product was created in a nonstandard way, without the operation process and the software development good practices. From 2010, it begun to standardize the process adopted for the company operation, unifying the operational team and standardizing the code and components of the system. In this process, it has generated a huge rework to rebuild the components of the system. "I don't have doubts that all this work was a payment of Technical Debt", says the responsible for the product. And to him, these Technical Debts might be necessary, because if the company has not had a fast launching of the product in the occasion, maybe it could not have got positioned in the market in such a solid format as it has been now.

c) Domain platform project

The project started in April 2008 by the company initiative to enter in the market to sell web domains. A third party company conducted operations and the original product development took 6 months until the first deployment. After the delivery of the first software release, the company created a development team for product evolution. It is based on Java, PHP and Perl technologies. Since the first release, changes in the software consisted only in adaptations and layout settings improvements. These adaptations were being added to the product without planning, making the software maintenance quite complex.

\subsection{Step 1. Contextualization interviews}

In order to collect contextual characteristics of the project, we performed a series of semistructured interviews, one for each for project team leader, aiming to take some project characteristics into account such as the history of each project and previous knowledge/decisions related to Technical Debt already taken by each the team. In this step, we used the following interview protocol:

1. How many professionals work in your team and how many years of experience each one has?

2. Could you describe the professional profile of each team member?

3. How much time is this team working together? How much time has your process being applied in the company? 
4. When did the project start?

5. Is it a project for the development of a product not yet released or it is a maintenance project?

6. What is the average time of each Sprint?

7. Describe the involvement of the Product Owner in the software development process.

8. How the team prioritizes tasks/histories?

9. How is quality handled throughout the product development life cycle?

10. Do you consider your team has previous knowledge in the concept of Technical Debt? If it does, how it is managed throughout the project life cycle?

\subsection{Step 2. Concept alignment}

For a second step, we performed interventions on the software development teams in order to align the concept of Technical Debt and explain the purposes of the study. For each reason, we performed a specific presentation to the teams. First, in order to align the concept of Technical Debt, we performed a 45 minutes presentation based on a material [Seaman 2013] provided by a specialist in Technical Debt - Dr. Carolyn Seaman. Second, we performed a 30 minutes presentation about the study in order to answer questions and promote research collaboration with the team members of each project.

\subsection{Step 3. Identifying Specific Processes}

In order to acknowledge differences in the process of each project, we participated in planning meetings of each team in each project. We participated for three Sprints in each project. We decided to use the Spring planning meeting since it is traditionally an environment in which all team members discuss next steps of evolution. During these meetings, we could perceive aspects such as tools used by the team throughout the Sprints and how software quality is addressed or not by team members. By participating in such meetings, we were able to collect different opinions from team members about the process used by the team. At the same time, in order to extend the conceptualization of Technical Debt, we promoted discussions during the meetings on how this concept might be related to tasks to perform during the next Sprint.

\subsection{Step 4. Framework proposal}

As a forth step, we proposed a framework for managing Technical Debt based on the feedback received by all three projects. This proposal was also based on works by Seaman [Seaman 2012] which argues that Technical Debt management should embrace three major steps: 1. Identifying Technical Debt; 2. Estimating Technical Debt; and 3. Taking a decision how to act in each case.

\subsection{Step 5. Data collection}

In order to help in answering the research question, we collected data from each team, further analyzed using qualitative coding procedures. We collected this data by applying a survey to team members after a few Sprints using the proposed model to manage Technical Debt. We designed the survey trying to collect the perception of team members on concept 
of Technical Debt, the effectiveness of the proposed model and possible effects regarding quality and productivity. The survey protocol is available as follows:

1. Did the concept of Technical Debt become clear for you? Do you think that your team may be able to produce software with fewer defects with it?

2. By identifying Technical Debt, did the team manage to put more effort to develop in new features instead of putting in paying debts?

3. Do you believe that using tools (Jira - in this case) makes it easier to manage Technical Debt?

4. Having the proposed model in hands, do you believe that from now on your team will produce software with less defects and higher quality?

By applying the survey, we managed to gather data in order to understand the effects of deploying this model for managing Technical Debt. To analyze it, we used open and axial coding procedures described by Strauss \& Corbin [1998]. Their proposal for these two qualitative coding procedures can be briefly summarized as:

- Open coding: The researcher passes by all data, identifying the core meaning of each part of data and applies a name - a code - to each part. Thereafter, the researcher groups codes into concepts and raise up larger groups - categories to represent the data in a higher level of abstraction.

- Axial coding: After identifying categories, the researcher finds relationships among them by returning to raw data and identifying such links.

- Selective coding: A central category is elected while related categories are emphasized in the study.

We chose NVivo

(http://www.qsrinternational.com/products_previous_products_nvivo8.aspx), as a tool for helping in the coding procedures while it is often used by social researchers for coding and analyzing qualitative data.

\section{Collected Data}

After the step of collecting information on how each team works, we made suggestions to the teams on how to highlight and control Technical Debt in the sprints.

Carolyn Seaman [2012] set up a framework for Technical Debt management in which the identification of the debt should be performed in three steps: identification of the debt, estimation debt and decision making on what to do.

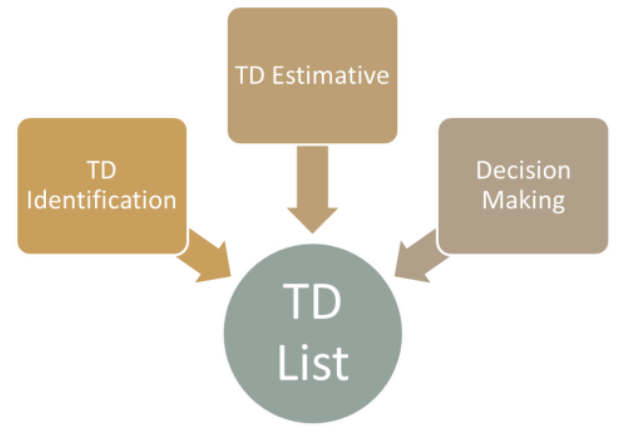

Figure 2. Technical Debt management framework 
Based on this framework, a proposal was made for the teams. This proposal was presented in a meeting held with the responsible of the three teams and we proposed some modifications in the Jira tool and some forms of control. This proposal was suggested to highlight and control the Technical Debt in the product backlog. Some parameterizations proposed, in the Jira, are fundamental to the proposal effectiveness:

- Story type: Every story created in a tool has type identification. Existing types, so far, were improvement and bug fix. One more type was added: Technical Debt.

- When a Technical Debt story is selected, some options appear in the type of debt (architecture, bug, testing and documentation).

- Also, some fields have to be completed to insert the value of the interest of the Technical Debt, in case it is not paid and another to define the probability of occurrence (high, medium and low).

Below there is the model defined in Jira for the Technical Debt control:

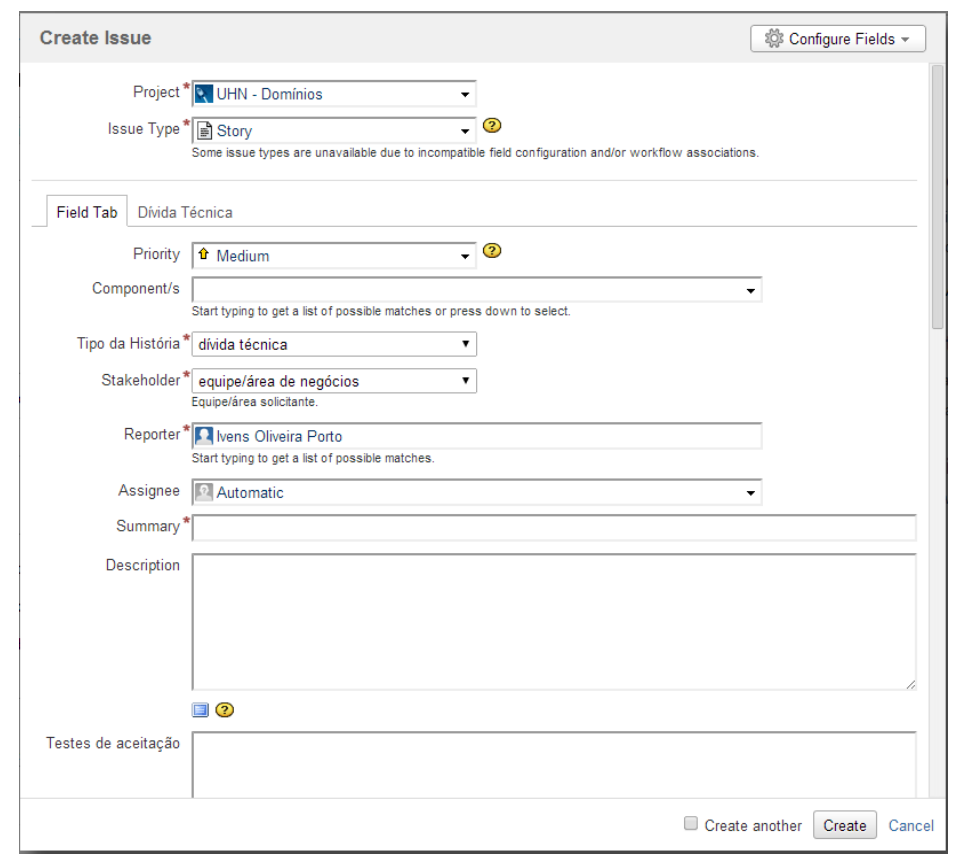

Figure 3. Creating a Story using Issue Tracking Tool

- Technical Debt Story: When we have a story in the sprint planning specifically about Technical Debt, include also its category;

- Technical Debt Type: When selected Technical Debt, include the type of debt (Architecture, bug, tests and documentation);

- The amount of Interest to each debt: how much it would cost to pay it now.

- The probability of Interest (High, Medium and Low): this metric is whether the team or developer believes it can happen again.

In the moment you have a Technical Debt mapped/identified, it can be estimated both in the sprint planning's and estimation meetings, along with other team stories, and properly prioritized by the Product Owner, according to their criticality and project development impact. 
After this identification, it will be possible to collect metrics regarding Technical Debt incidence in the team development process, how it affects them and also with relation to development costs for the company (based on the allocation of the team in the development process or Technical Debt work).

Another point would be included in a whiteboard, where the teams controlling the daily meetings stories, an area for the team members include Technical Debts found during the sprint. In team meetings with the PO, the Scrum Master takes these suggestions to be inserted into Jira and evaluated by the team and PO.

These controls aims to highlight for the team and the Product Owner, the occurrence of Technical Debt, show it at sprint planning meetings and let clear their incidence and impact in the development process.

\section{Results}

The resulting model is shown in Figure 4.

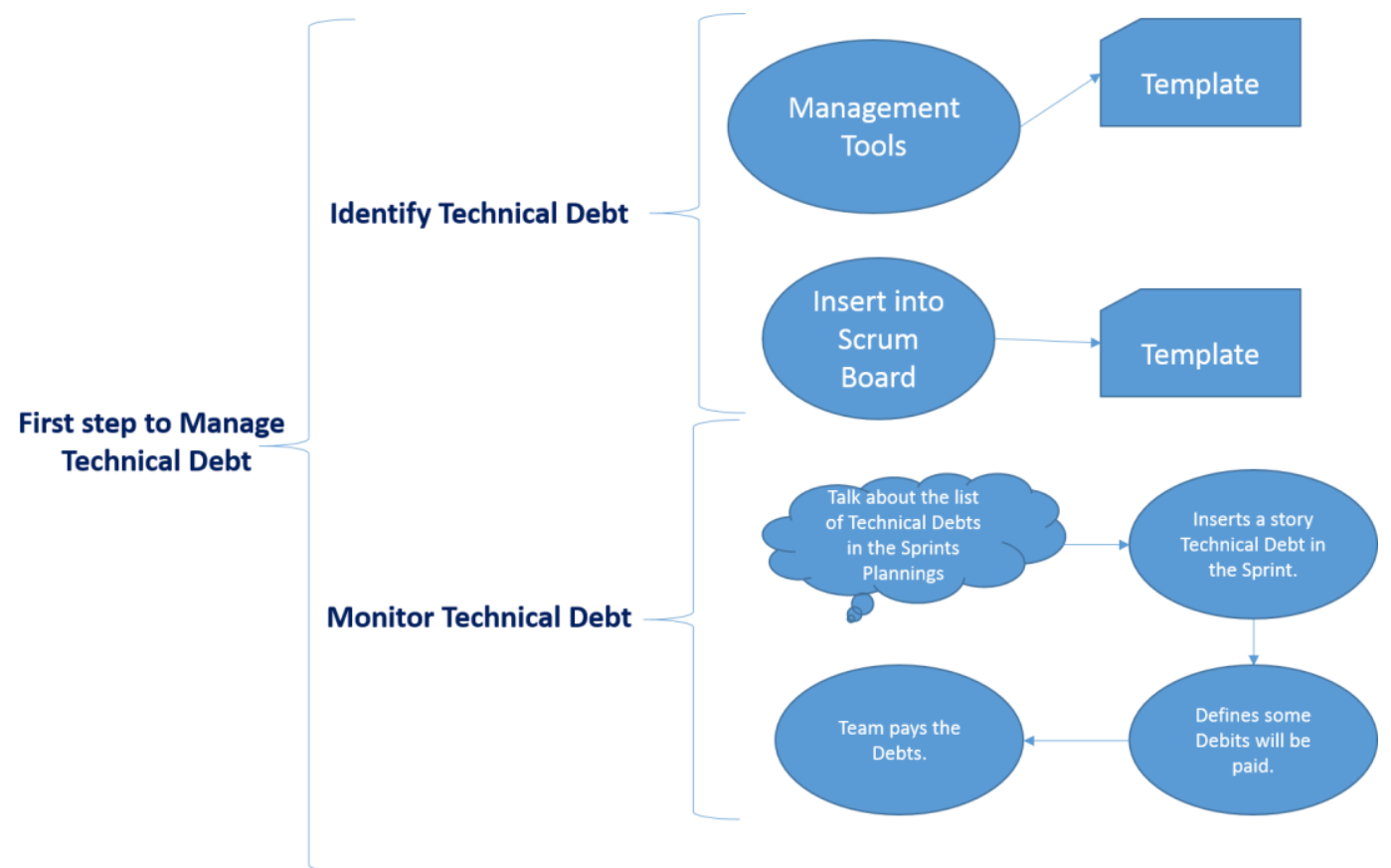

Figure 4. Model to Identify and Monitor Technical Debt

When the team start to identify Technical Debt, it is possible to use the tools of project management to register the list of Technical Debt, given it is only necessary to create a template with fields that must be filled on a first in which the team will register the debt. Then we suggested that in some Sprints the team talk about the list of Technical Debts and decide whether it is necessary to insert a story to pay off some debts techniques. If the team decide paid, then select the debts to be paid and place them on the Scrum board so that any team member to pay. 


\subsection{Benefits for the team}

The study identified some benefits generated for the team after the implementation of the model. Below we describe the results after each step of the data analysis.

In the open coding analysis, the codes were defined and some examples of these codes are listed in Table 1.

\begin{tabular}{|l|r|}
\hline \multicolumn{1}{|c|}{ Conceptual category } & Ocurrences \\
\hline Thecnical debt management & 11 \\
\hline quality enhancement & 10 \\
\hline Techical debt definition & 10 \\
\hline history documentation & 10 \\
\hline Positive points with techcnical debt & 9 \\
management & 8 \\
\hline Techinical debt visibility & 8 \\
\hline Negative points with techcnical debt & 8 \\
management & \\
\hline Technical debt payment & \\
\hline
\end{tabular}

\section{Table 1. Preliminary codes}

The code Technical Debt management had more evidence in the survey answered by team members, highlighting the relevance of the topic: "...Once recognized and analyzed the Technical Debt stories, it is possible to make explicit software problems and identify critical points in the system quality process...".

Others codes very mentioned were quality enhancement and Technical Debt definition. As reported by team members, managing Technical Debt makes the teams produce software with best quality and team members assimilated the concept and benefits of managing Technical Debt.

In the second step, the codes were rearranged in a level of greater abstraction. Thus, new combinations are again set to form the categories that, in turn, are organized. Below in Figure 5 we represent the categories:

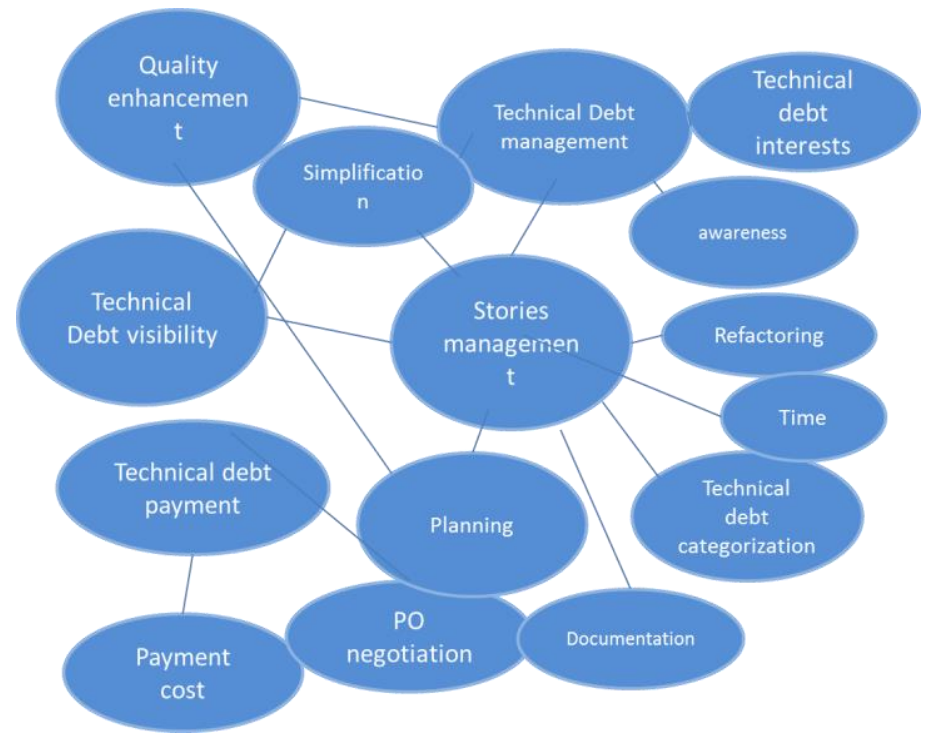

Figure 5. Relationship among categories 
The model implemented, was based on the tools and processes existing in the company. After implementation, the team members now have greater awareness to incur new Technical Debts and greater visibility over it: "... Now the team exposes to PO the Technical Debt existing in the software and these stories are performed by team with the stories of improvement and evolution of the product...". The model facilitates the management of the stories with others that were already treated by the team, providing the members measured the cost of payment of Technical Debt and interest payments associated Technical Debt.

The stories management model led teams to better planning, a proper documentation with a better categorization of stories that are Technical Debt and estimate the time and priority in payment ".... Stories for Technical Debt solutions were incurred and prioritized according to the cost of your payment, with the stories of product improvements...". This documentation allows better control and better negotiation with the Product Owner to prioritize Technical Debt stories "... Now the team exposes to Product Owner the Technical Debt that exist in the software and these stories are performed by the team along with the stories of improvement and product evolution...".

With Technical Debt control the teams can better plan for future refactoring of the product without acquiring new debt. Managing Technical Debt brings an improvement in software quality: "... You can make explicit points of software problem and identify critical points in the quality of an informed way system, helping to prioritize and correct problems, which optimizes search for a better quality in the system..."

In the last data analysis the categories were refined and integrated unveiling a category deemed as central, pervading all the others in this case, the objective is not to generate a theory, but instead to identify the main categories, the principal influence to identify Technical Debt. The mains result is describe in Figure 6:

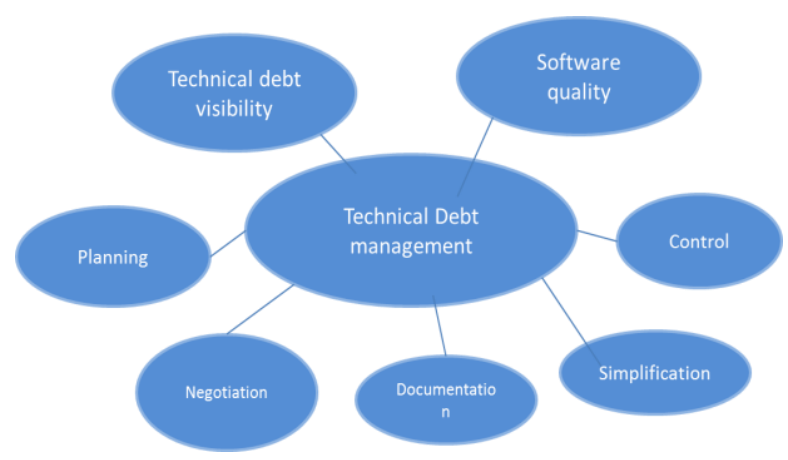

Figure 6. Selected categories

The categories of Technical Debt Management, visibility of Technical Debt, planning, negotiation, documentation, simplicity, control and software quality are the major influences on the teams in the model implemented. Each category is part of the results, although none of them shows completely. For this reason, abstract categories, a conceptual idea under which all categories are included are necessary. For this reason, we conclude that the resulting main category is "Technical Debt Management". It was clear to all teams that identify and manage Technical Debt brings most visibility in the development process. 
These Technical Debts documented in Jira tool, are transformed into stories and prioritized along with other product enhancements stories in a negotiation process with the Product Owner, making the product more quality. All this management model and stories control, was defined based on a simple way in which the processes and systems preexists were maintained without impact to the team and without significantly changing the way of working.

The entire model created and implemented, intends to implement a control Technical Debt and interest associated with the product, and thus, increasingly improve the quality of software delivered by development teams.

\section{Conclusion}

It was clear to team members about the importance and benefits of identifying and managing Technical Debt. All participants agreed that Technical Debt identification and management directly affect the quality of the product. Another point to consider is that most members of the teams had never heard about the concept of Technical Debt or just knew intuitively. Now they always think about the necessity of acquiring Technical Debt, and often decide not to do it. They always think and discuss about Technical Debt contraction in the stories to be implemented. And this implies the creation of a culture focused on quality.

Another point well considered, was the simplicity of the process implemented with minor modifications on the existing tool. Technical Debt stories are registered in the tool with other stories, even though this increase the team work to document the stories.

According to Eisenberg (2012), Technical Debt management is very important for software developers, especially in agile methods projects. This can be attributed to the changing nature of the code in agile development, the expectation for continuous refactoring, and the need for continuous integration [Eisenberg, 2012] and this research was conducted in a company that uses agile in their development process.

It is also worth mentioning that the main idea of this model is to verify that each iteration is working in the model, and what is not, and then evolve/change whatever it takes.

\section{Threats to validity}

The research data were collected through interviews and recordings, so, for a future version, would be interesting to perform data triangulation seeking to confront the evidence with data from project documents.

\section{Future work}

We aim to continue this research, replicating the proposal to other company projects. We also intend to replicate interviews and monitoring the records of Technical Debt in Jira tool to gather more information about the pros and cons of identifying and monitoring Technical Debt.

Additionally, we will suggest and insert new ways and management tools for Technical Debt management and replicate to other teams in the company. Also, other studies with similar objectives are being developed in other companies in São Paulo. 
Regarding the Technical Debt, there are still many gaps in how to identify, manage and use it, strategically throughout the project. And, also there is not a complete theory about Technical Debt.

\section{References}

Cunningham, W. (1992) "The WyCash Portfolio Management System". Conference on Object-Oriented Programming Systems, Languages, and Applications (OOPSLA'92). Vancouver: [s.n.].

Brown, N. et al. (2010). Managing technical debt in software-reliant systems. The FSE/SDP workshop on Future of software engineering research. New Mexico: [s.n.]. p. 47-52.

Gartner. (2012). "Gartner Estimates Global 'IT Debt' to Be \$500 Billion This Year, with Potential to Grow to $\$ 1$ Trillion by 2015." http://www.gartner.com/it/page.jsp?id=1439513 . Last access April/2012.

Glaser, B and Strauss, A, L.(1967). The Discovery of Grounded Theory: Strategies for Qualitative Research. Aldine Publishing Company, Chicago, IL, USA.

Guo, Y. (2009) "Measuring and Monitoring Technical Debt." 4th International Doctoral Symposium on Empirical Software Engineering. [S.l.]: [s.n.]. p. 256.

Guo, Y. and Seaman, C. (2011) "A portfolio approach to technical debt management." Proceeding of the 2nd working on Managing technical debt. [S.l.]: [s.n.].

Guo, Y, Seaman, C. Gomes, R. Cavalcanti, A. Tonin, G. et.al (2011) "Tracking Technical Debt - An Exploratory Case Study". Proceedings of the 27th IEEE International Conference on Software Maintenance (ICSM' 11), 528-531, 2011.

Highsmith, J. (2011). “Accelerating Enterprise Agility Adaptive Leadership." Agile Conference 2011.Salt Lake City, Utah, August.

Seaman, C., et.al. (2012) "Using Technical Debt Data in Decision Making: Potential Decision Approaches." MTD2012: 3rd Workshop on Managing Technical Debt, at ICSE 2012, Zurich, Switzerland.

Seaman, C. (2013) Model to Identify and Management Technical Debt. Available in: http://www.technicaldebt.umbc.edu/. Access: 02/08/2014.

Strauss, A. and Corbin, J (1998). Basics of Qualitative Research: Techniques and Procedures for Developing Grounded Theory. ISBN: 9780803959408

VersionOne. (2011). "State of Agile Development Survey Results" http://www.versionone.com/state_of_agile_development_survey/11/. Last access April/2012.

Williams, L. (2010) "Agile Software Development Methodologies and Practices". Advances in Computers, vol. 80, pp. 1-44.

Yin, R. K. (1989) “Case Study Research: Design and Methods.” 3. ed. [S.1.]: Sage Publications. 
Appendix C

Short Paper published at Agile

Conference, 2014 


\section{Technical debt management: Where to start from?}

\section{Preliminary experiences from an action research study}

\author{
Graziela Tonin, Alfredo Goldman \\ Department of Computer Science \\ University of São Paulo \\ São Paulo, SP, Brazil \\ \{grazzi, gold\} @ime.usp.br
}

\author{
Vinícius Suyama, Nara Sakamoto, Danilo Saita, Enderson \\ Bazetti, Danilo Possarle, Viviane Santos \\ MAPS Solutions and Services SA \\ São Paulo, SP, Brazil \\ \{vinicius.suyama, nara.sakamoto, danilo.saita, enderson.bazetti, \\ danilo.possarle, viviane.santos\}@MAPS.com.br
}

\begin{abstract}
Technical Debt (TD) involves bad design and implementation decisions that negatively impact code quality and maintenance. Despite our best intentions, TD is often a major concern in our systems. Because of that, this paper presents an action research study on technical debt from two agile teams of a software development company. These teams aimed at applying approaches to deal with TD identification, monitoring, and management. These three aspects were conducted in three action research cycles respectively for both teams. By getting more visibility about the problems on their code, the teams became more aware of the importance of this topic. Likewise, they started to build a sense of responsibility for coping with TD.
\end{abstract}

Keywords-Technical debt; informative workspace; visibility; monitoring, tools, management; pay back; action research.

\section{INTRODUCTION}

Technical Debt is a metaphor developed by Cunningham [1], which makes an inference to a financial debt. Every time an immature code is inserted in the project, a debt is contracted and should be paid in the form of code rewriting.

Some studies are being conducted in this area to formalize a theory about this [2][3]. Moreover, other studies try to find ways that can help practitioners to identify and manage TD [4][5][6][7], however, we still lack formal evidence on how to effectively use this concept.

We conducted an action research (AR) with two teams (termed as Team A and Team B) of a software development company of the financial sector. The mentioned company is a medium-sized Brazilian company with about 200 employees, being 150 of software and products' development teams.

Team A is responsible for the company own framework developed by their software developers to leverage the company products. Because of that, Team A is also concerned about managing the framework TD. Immature code, such as coupled code, high complexity, duplicate code and missing tests are the common problems they identify in the submitted pull requests. At Company context, some pull requests are merged to the framework even containing bad code, usually because teams have other priorities that prevent them to fix bad code.

Team B is responsible for some of Company's products. They usually work with tight schedules and have a high rate of product version delivery. The team often prioritizes feature delivery over code quality, which often means poorly developed code, lack of automated tests and overall dissatisfaction with the product's quality. On top of that, Team B has difficulty coping with well-defined development process.

Thus, the main aim of this study is to answer the following research question: "How to deal with technical debts in a software development company?" The research method used in this study is action research [8], because of mutual interest on the research topic. Thus, members of the organization under study are actively engaged in the quest for information and ideas to guide their future actions.

This paper describes preliminary results from three AR cycles for both teams. These teams applied specific approaches to dealing with technical debts and experienced the benefits of this endeavor. Both teams strived to identify, monitor and manage technical debts.

\section{EXPERIENCE FROM BOTH TEAMS}

Team A aimed at increasing the visibility of the framework's TD, monitoring $\mathrm{TD}$, and to make them part of their team product development process. Table 1 presents a summary of each AR step for the three cycles of Team A. Team B's main concern is the actual decrease in product's quality through recurring bugs. This team began to look for ways to know their TD. Table 2 presents a summary of each AR step for the three cycles of Team B.

TABLE I. SOURCES OF EVIDENCE COLLECTED IN THIS STUDY

\begin{tabular}{|c|c|c|c|}
\hline AR Step & $\begin{array}{l}\text { 1st Cycle } \\
\text { Provide visibility of TD in the framework }\end{array}$ & $\begin{array}{l}\text { 2nd Cycle } \\
\text { Monitor and anticipate TD }\end{array}$ & $\begin{array}{l}\text { 3rd Cycle } \\
\text { Management and systematization of TD payment }\end{array}$ \\
\hline Diagnosing & $\begin{array}{l}\text { Several technical debts being incorporated } \\
\text { to the company framework. }\end{array}$ & $\begin{array}{l}\text { The need to monitor and anticipate } \\
\text { TD. }\end{array}$ & $\begin{array}{l}\text { The need to systematize TD pay off and motivate other } \\
\text { teams to care about their TD, as a way to improve overall } \\
\text { code quality. }\end{array}$ \\
\hline $\begin{array}{l}\text { Action } \\
\text { Planning }\end{array}$ & $\begin{array}{l}\text { Provide visibility of the framework } \\
\text { technical debts through tools. }\end{array}$ & $\begin{array}{l}\text { Develop a tool to analyze pull requests } \\
\text { and automate the pull requests analysis } \\
\text { process. }\end{array}$ & $\begin{array}{l}\text { Team A selected relevant metrics from the SONAR tools, } \\
\text { such as unit tests coverage and TD in days. }\end{array}$ \\
\hline $\begin{array}{l}\text { Action } \\
\text { Taking }\end{array}$ & $\begin{array}{l}\text { A new field was created in the issue tracker } \\
\text { tool to indicate that it was a technical debt. } \\
\text { SonarQube server was installed at a Team } \\
\text { A's member computer for a testing stage. }\end{array}$ & $\begin{array}{l}\text { The issue tracker was configured to } \\
\text { trigger a parameterized Jenkins job to } \\
\text { execute the Pull Request Analyzer } \\
\text { when a pull request is submitted to } \\
\text { Team A analysis. }\end{array}$ & $\begin{array}{l}\text { SONAR metrics allowed to identify the recurring issues } \\
\text { and take actions to prevent them through pair } \\
\text { programming (PP), code review and test driven } \\
\text { development (TDD). Team A considered incorporating TD } \\
\text { to their software process. We also made short presentations } \\
\text { to other teams in the company regarding Team A } \\
\text { experience coping with TD. }\end{array}$ \\
\hline Evaluation & $\begin{array}{l}\text { TD list presented to the team in daily } \\
\text { meetings. In a team retrospective, the team } \\
\text { members discussed the visibility of the TD } \\
\text { and approved the installation of SonarQube } \\
\text { tool on a production server. }\end{array}$ & $\begin{array}{l}\text { With this tool, Team A could establish } \\
\text { a reliable level metric to show } \\
\text { reliability of the pull requests. This } \\
\text { metric helped Team A in deciding the } \\
\text { prioritization of analysis of pull } \\
\text { requests. }\end{array}$ & $\begin{array}{l}\text { After applying the techniques, Team A verified significant } \\
\text { improvements to their quality metrics. With short } \\
\text { presentations, they got valuable feedback from the other } \\
\text { teams regarding TD, such as the need to identify our own } \\
\text { patterns for dealing with TD in the context of the company } \\
\text { and document them. }\end{array}$ \\
\hline
\end{tabular}




\begin{tabular}{|l|l|l|l|}
\hline & $\begin{array}{l}\text { Visibility allowed them to understand the } \\
\text { location of TD, to define points of }\end{array}$ & $\begin{array}{l}\text { By observing the TV monitor, Team A } \\
\text { members were able to monitor critical } \\
\text { LD and take proper actions, such as }\end{array}$ & $\begin{array}{l}\text { Automating pull requests analysis process through a proper } \\
\text { requests and convince developers to worry about code } \\
\text { attention, to prioritize TD by severity, }\end{array}$ \\
$\begin{array}{l}\text { present TD to the responsible team(s) as a } \\
\text { way to become aware of relevant TD. }\end{array}$ & $\begin{array}{l}\text { requesting responsible teams to pay } \\
\text { down their TD or to provide guidance } \\
\text { and training on recurring problems. }\end{array}$ & $\begin{array}{l}\text { other teams the importance of paying down their TD are } \\
\text { crucial concerns to provide product health. }\end{array}$ \\
\hline
\end{tabular}

\begin{tabular}{|c|c|c|c|}
\hline AR Step & $\begin{array}{c}1 \text { st Cycle } \\
\text { Provide visibility of TD regarding tests }\end{array}$ & $\begin{array}{c}\text { 2nd Cycle } \\
\text { Categorize TD }\end{array}$ & $\begin{array}{c}\text { 3rd Cycle } \\
\text { Encourage to register and pay off TD }\end{array}$ \\
\hline Diagnosing & $\begin{array}{l}\text { This team diagnosed that their code was being } \\
\text { produced lacking automated tests and proper time to } \\
\text { take actions to prevent TD. }\end{array}$ & $\begin{array}{l}\text { The need to collect more information } \\
\text { about each TD item to facilitate when } \\
\text { they would pay them off. }\end{array}$ & $\begin{array}{l}\text { They realized the team members started to } \\
\text { submit fewer TD than in previous cycles. }\end{array}$ \\
\hline $\begin{array}{l}\text { Action } \\
\text { Planning }\end{array}$ & $\begin{array}{l}\text { Two team representatives planned to disseminate } \\
\text { the concept of TD to their members. Together, they } \\
\text { defined what types of TD could be identified and } \\
\text { how. }\end{array}$ & $\begin{array}{l}\text { Team B planned to define forms to show } \\
\text { the real importance of identifying } \\
\text { technical debt. They added information, } \\
\text { such as context and initial analysis. They } \\
\text { also decided to temporarily allocate a } \\
\text { team member to pay their TD items. }\end{array}$ & $\begin{array}{l}\text { They planned to select metrics, analyze the } \\
\text { code with SONAR tools, encourage all team } \\
\text { members to pay down TD, and adopt PP. } \\
\text { Finally, they also planned to make short } \\
\text { presentations along with Team A and R\&I } \\
\text { department about their experience. }\end{array}$ \\
\hline $\begin{array}{l}\text { Action } \\
\text { Taking }\end{array}$ & $\begin{array}{l}\text { Because of time constraints, Team B members } \\
\text { decided to focus on one single type of TD to track } \\
\text { for, which was TD regarding tests. A board was } \\
\text { created to stick TD notes and corresponding TD } \\
\text { issues in their issue tracker tool. }\end{array}$ & $\begin{array}{l}\text { Team B started to compare estimate time } \\
\text { for TD related to tests and consider them } \\
\text { in the project planning. One team } \\
\text { member was concerned in paying their } \\
\text { TD items. }\end{array}$ & $\begin{array}{l}\text { They paid down TD items and adopted PP. } \\
\text { They started the analysis of the issue tracker } \\
\text { reports and SONAR tools as a way to } \\
\text { identifying the recurring issues. }\end{array}$ \\
\hline Evaluation & $\begin{array}{l}\text { The board provided additional reminders for the } \\
\text { developers about what information must beg filled } \\
\text { when registering technical debt items. }\end{array}$ & $\begin{array}{l}\text { Team B understood the weaknesses of } \\
\text { their application, which improved their } \\
\text { awareness on the TD subject. }\end{array}$ & $\begin{array}{l}\text { They were more aware that getting TD is not } \\
\text { necessarily bad, but coping with TD in the } \\
\text { software development process is a challenge. }\end{array}$ \\
\hline Learning & $\begin{array}{l}\text { With the TD board, it was possible to get visibility } \\
\text { and preliminarily evaluate the health of the code. } \\
\text { Team members are more aware of code quality. }\end{array}$ & $\begin{array}{l}\text { Detailed TD information helped in } \\
\text { paying them down. }\end{array}$ & $\begin{array}{l}\text { They realized that collaboratively monitoring } \\
\text { TD through tools and adopting PP periodically } \\
\text { improved their behavior on this issue. Team } \\
\text { members, leader and project manager are now } \\
\text { more supportive of TD. }\end{array}$ \\
\hline
\end{tabular}

\section{RECOMMENDATIONS}

With the approaches adopted by both teams, they experienced a decrease in TD items and understood the importance of dealing with TD. It is expected that TD becomes manageable over time. This research suggests ways to make TD explicit, as well as monitoring and managing TD. Below we provide a summary of recommendations extracted from this AR study.

\section{1) TD Visibility}

Getting technical debt visibility is the first step on its management. There are several static code analysis tools, such as SonarQube that can provide code quality information. This type of tool allows the team to identify possible bugs and points of attention in the code that are sensitive to changes.

Also, TD must be registered. The best way to do it is using an issue tracker such as JIRA. The register must have the debt description and who created the debt. A created vs resolved debts chart can be created and incorporated to the team monitor TV. This approach allows the teams to identify and prioritize critical debts to be paid.

\section{2) TD Monitoring}

The next step is monitoring a group of metrics the team consider important, such as unit tests coverage and complexity. These information must be visible to the team in order to allow its monitoring by the team. Displaying relevant information on a large TV monitor is one way to accomplish this.

\section{3) TD Management}

Both teams fostered the adoption of programming techniques to pay down TD, such as PP, code review and TDD. They also influenced other teams in the company through short presentations about their experience.

4) Incorporating technical debt concerns in teams' software development processes

After consolidating the TD management, it is necessary that TD is incorporated into team's software development process. Technical debts must be incorporated to the team's backlog and their payment should be considered as a story on the team's process. The team may separate TD according to their criticality and choose which of them will be paid on the next sprint. TD also should be part of teams' goals.

\section{ACKNOWLEDGMENTS}

We are grateful to the company for providing access to its environment, and also to the employees participating in this study who readily volunteered their time to contribute to this research.

\section{REFERENCES}

[1] W. Cunningham, "The WyCash Portfolio Management System," in Addendum to the proceedings on Object-oriented programming systems, languages, and applications, 1992, pp. 29-30.

[2] G, Rebeka. "Characterization and Theoretical Conceptualization of the Technical Debt Metaphor Through an Exploratory Study.” Master's Dissertation in the Federal University of the Pernambuco, September, 2011.

[3] B. N. et al. "Managing technical debt in software-reliant systems." The FSE/SDP workshop on Future of software engineering research. New Mexico: [s.n.]. p. 47-52.

[4] G.Yepu. and S. Carolyn . "Measuring and Monitoring Technical Debt." 4th International Doctoral Symposium on Empirical Software Engineering. [S.1.]: [s.n.]. p. 25-46. 2009.

[5] G.Yepu. and S. Carolyn. "A portfolio approach to technical debt management." Proceeding of the 2nd working on Managing technical debt. [S.1.]: [s.n.]. 2010.

[6] G, Yepu, S, Carolyn. G, Rebeka. C. Antonio. T. Graziela. et.al "Tracking Technical Debt - An Exploratory Case Study". Proceedings of the 27th IEEE International Conference on Software Maintenance (ICSM' 11), 528-531, 2011.

[7] T. Graziela. "Decisions in Projects Software Development and Technical Debt Metaphor: An Exploratory Case Study". Master's Dissertation in the Federal University of the Pernambuco, September, 2011.

[8] Baskerville, "Investigating information systems with action research", Communications of the Association for Information System, Volume 2, Article 19, October 1999. 
Appendix D

Paper published at Brazilian Workshop of Agile Methods, 2012. 


\title{
Uma Análise de Dívida Técnica em uma Empresa de Tecnologia com Desenvolvimento baseado em Scrum
}

\author{
Graziela S. Tonin ${ }^{1}$, Rogério Chaves ${ }^{2}$, Alfredo Goldman'1, Viviane Santos ${ }^{1}$. \\ ${ }^{1}$ Instituto de Matemática Estatística e Ciência da Computação - Universidade de São \\ Paulo (USP) \\ Rua do Matão, 1010 Cidade Universitária - São Paulo - SP - Brazil \\ ${ }^{2}$ Instituto de Pesquisas Tecnológicas do Estado de São Paulo (IPT) \\ \{grazzi, gold, vsantos\}@ime.usp.br, rcpires@uoldiveo.com
}

Resumo. Até o momento existem muitas lacunas nas pesquisas científicas sobre Dívida Técnica. Assim como sobre o seu comportamento ao longo dos ciclos de vida dos projetos de software. O que as pesquisas vêm destacando é que Dívida Técnica existe e precisa ser considerada no momento de tomada de decisão. Por outro lado, tem ficado claro a eficiência e eficácia dos princípios e valores ágeis se aplicados e seguidos no gerenciamento de desenvolvimento de software. Por isso, o objetivo deste trabalho é verificar como se dá o comportamento de Dívida Técnica e seu impacto no decorrer de um projeto, em uma empresa com alto grau de maturidade em Métodos Ágeis, no caso Scrum. Para isso, realizou-se um estudo de caso em uma empresa líder de mercado no setor de tecnologia e algumas Dívidas Técnicas de impacto em determinado projeto foram identificadas e analisadas. Como resultado, ficam claras as razões pelas quais Dívidas Técnicas são contraídas, suas influências, motivações e impactos. Um modelo foi gerado e pode ser utilizado em outros projetos como base no momento de tomada de decisão para que a área de negócio não tenha que dispensar recursos com problemas recorrentes, podendo assim redirecionar os investimentos para a evolução do sistema.

Palavras Chaves: Dívida Técnica; Tomada de Decisão Estratégica; Scrum.

\begin{abstract}
So far there are few scientific studies about Technical Debt and their behavior over the software project life cycles. However, some works are outlining that Technical Debt exists and needs to be considered at the time to business on decision making. Moreover, it has become clear the efficiency and effectiveness of agile principles and values when applied and followed in managing software development. Therefore, the goal of this paper is to verify how is the behavior of Technical Debt and its impact over a project lifecycle in a company leader in the technology sector with a high degree of maturity in Agile Methods, Scrum in this specific case. For this, we performed a case study in a market leader in the technology sector and some Technical Debt impact on a project were identified and analyzed. As a result there are clear reasons why Technical Debts are contracted, influences, motivations and impacts. An important model that can be used in other projects based at the time of decision making so that the business does not have to dispense resources with recurring problems, so you can redirect investment to the evolution of the system.
\end{abstract}

Keywords: Technical Debt; Strategic Decision Making; Scrum. 


\section{Introdução}

Dívida Técnica é uma metáfora criada por Cunningham (1992) que em seu relato de experiência do OOPSLA 1992 criou a primeira definição para o termo:

“... A primeira vez que a qualidade do código é comprometida é como se estivesse incorrendo em Dívida Técnica. Uma pequena Dívida Técnica acelera o desenvolvimento até que seja paga através da reescrita do código. O perigo ocorre quando a Dívida Técnica não é paga. Cada minuto em que o código é mantido em inconformidade, juros são acrescidos na forma de reimplementação..."

Esta metáfora faz inferência a dívida financeira e vem ganhando força nos últimos anos. Onde, por uma decisão estratégica durante o processo de evolução de um sistema a qualidade do código é potencialmente comprometida devido a restrições impostas ao processo, como por exemplo, tempo/recursos. Também muitas vezes os desenvolvedores optam por comprometer a qualidade do software, para atender alguma demanda urgente em outra dimensão do sistema, como por exemplo, time to market. É como se estivessem contraindo uma Dívida, que precisará ser paga no futuro. $\mathrm{Na}$ analogia, isso implica em juros que podem crescer na forma de tempo, esforço ou custo extra de futuras alterações [Guo 2009].

A mesma tem sido tema de muitas discussões em blogs, fóruns, palestras e eventos. Por um lado, há vários profissionais da área de desenvolvimento de software tentando descobrir uma forma de trabalhar este conceito em suas empresas. Por outro lado, algumas pesquisas estão sendo realizadas na área, visando formalizar uma teoria a respeito [Brown 2010 e Gomes 2011]. Além disso, outras pesquisas tentam encontrar formas que possam auxiliar a identificá-la e geri-la [Guo 2009; Guo 2011; Guo 2012 e Tonin 2011], porém, ainda faltam evidências formais sobre como usar de forma eficaz o conceito de Dívida Técnica. O que pesquisas recentes mostram, é que se gastou com Dívida Técnica em 2010 aproximadamente U\$500 bilhões e estima-se que até em 2015 o gasto chegará a U\$1 trilhão [Gartner 2012].

Em contrapartida, pesquisas relatam que cada vez mais, nos últimos 10 anos, empresas têm adotado Metodologias Ágeis, como forma de gerir projetos de desenvolvimento de software. E que sua eficácia e benefícios têm sido significativos, onde os resultados apresentados relatam equipes mais produtivas, menos estressadas e clientes mais satisfeitos com os produtos entregues [VersionOne 2012 e Williams 2010]. Outras pesquisas evidenciam que agilidade é chave para o sucesso organizacional, onde estudos do MIT revelam que empresas Ágeis aumentam a receita 30\% mais rápido e geram 37\% mais lucros do que as não ágeis [HighSmith 2011]. Mas segundo HighSmith (2011), qualidade ou a falta dela continua ainda sendo a questão central para a agilidade eficaz.

Nas próximas seções será discorrido sobre os objetivos da pesquisa (Seção 2). $\mathrm{Na}$ seção 3, é relatado sobre a metodologia utilizada e como a mesma foi aplicada na coleta e análise dos dados. Na seção 4 os resultados são explanados onde um modelo foi criado para auxiliar a identificação e monitoração de Dívida Técnica. Na quinta seção os resultados são discutidos e comparados com os estudos existentes, coletados até o momento da escrita do artigo. 


\section{Objetivos da Pesquisa}

Esta pesquisa foi realizada em uma empresa de grande porte que adota Scrum como Metodologia de Gerenciamento de Projetos há mais de quatro anos. É o maior portal de internet do Brasil e provê uma gama de produtos e serviços que atendem o mercado de internet. A empresa foi fundada em 1996 como provedor de serviços em internet focando em conteúdo e serviços discados de internet. A partir daí foi diversificando a sua gama de produtos e serviços e evoluindo juntamente com as tecnologias e práticas adotadas no mercado de internet. Possui uma área de Pesquisa e Desenvolvimento (P\&D) que é responsável pela concepção, desenvolvimento e manutenção de todos os produtos e serviços providos pelo portal. Todo o desenvolvimento de software é baseado em Métodos Ágeis, com foco principal em Scrum.

Levando em conta que a empresa investigada segue esses princípios, buscou-se investigar como o conceito de Dívida Técnica é considerado no desenvolvimento de software. Sendo estes conceitos utilizados, procurou-se evidências para responder as seguintes questões:

(1) A empresa em estudo conhece o conceito de Dívida Técnica? Caso sim, como é considerado na gestão do projeto?

(2) Decisões Estratégicas implicaram na contração de Dívida Técnica?

(3) Qual foi o impacto ao longo do tempo?

\section{Metodologia Utilizada}

Realizou-se um estudo de caso exploratório, pois, segundo Merriam (2009) e Robson (2002) pesquisas qualitativas tem como foco principal entender o significado e/ou natureza de um fenômeno.

\subsection{Seleção da Amostra e Caso Estudado}

O caso estudado foi selecionado com base no método de amostragem intencional [Yin, 2003]. Esta abordagem é comumente utilizada, quando o objetivo da pesquisa é explorar, entender ou encontrar evidências sobre algo. Nesse método deve-se buscar por casos que possuam informações relevantes e abundantes para o problema de pesquisa. $\mathrm{O}$ mesmo tipo de amostragem foi utilizado para a seleção de pessoas que foram convidadas a realizar a entrevista. Logo, primeiro foi necessário encontrar um caso onde acreditava-se que haviam evidências de Dívidas Técnicas e que fosse passível de acesso devido a restrições dos negócios da empresa. Para isso, realizou-se conversas informais com o cliente de alguns projetos e com base em relatos do mesmo, definiu-se o caso a ser estudado.

\subsection{Entrevistas}

Optou-se pela realização de entrevistas, visto que em pesquisas qualitativas é um dos principais métodos utilizados na coleta de dados. Também não era permitido o acesso aos documentos do projeto, por se tratar de um projeto confidencial. Como foram investigados eventos passados, não seria possível observá-los. Por isso, foram realizadas 
entrevistas informais com um membro do time que participou do projeto desde o início. Nestas entrevistas foram identificados momentos de solicitações de grandes mudanças e/ou mudanças de grande impacto, ao longo do ciclo de vida do projeto. De posse desses casos, foi criado um roteiro de entrevista com perguntas fechadas e abertas, seguindo os passos sugeridos por Merriam (2009), realizando assim uma entrevista semiestruturada. Essa mesma metodologia tem sido utilizada em outros trabalhos [Guo 2012; Gomes 2011 e Tonin 2011].

$\mathrm{O}$ roteiro foi dividido em duas partes. Em um primeiro momento explicava-se para o entrevistado o motivo da pesquisa. Em seguida, uma breve explanação era feita sobre um determinado momento do projeto para verificar se a pessoa recordava do mesmo. Em um segundo momento, eram apresentadas as questões que foram divididas em três contextos:

- Caracterizar a função/papel da pessoa naquele dado momento do projeto.

- Identificar o que a pessoa recordava sobre aquele dado momento no projeto.

- Perguntas específicas sobre decisões tomadas, problemas enfrentados e impactos gerados, naquele momento e ao longo da evolução do sistema na visão do entrevistado.

Algumas perguntas eram adicionadas ao longo das entrevistas caso o pesquisador achasse necessário para deixar clara alguma informação adicional.

\subsubsection{Análise dos Dados}

Segundo [Strauss 2008], basicamente há três componentes principais na pesquisa qualitativa. Primeiro há os dados, que podem vir de várias fontes, tais como entrevistas, observações, documentos, registros e filmes. Depois, há os procedimentos, que os pesquisadores podem usar para interpretar e organizar os dados. Eles geralmente consistem em conceitualizar e reduzir os dados, elaborar categorias em termos de suas propriedades e dimensões e relacioná-las por meio de uma série de declarações preposicionais. Conceitualizar, reduzir, elaborar e relacionar sempre são referidos como codificação. Esses passos e princípios citados por Strauss foram seguidos para a análise e sumarização dos resultados. As entrevistas foram transcritas e conceitualizadas segundo suas propriedades e dimensões.

\subsection{Casos Estudados}

Os casos selecionados para serem investigados foram os seguintes:

CASO 1: Upgrade da versão do JQuery.

JQuery é uma biblioteca JavaScript cross-browser desenvolvida para simplificar os scripts client side que interagem com o HTML. JQuery é a mais popular das bibliotecas JavaScript utilizadas no mundo (http://trends.builtwith.com/javascript/JQuery). No início do projeto era utilizada a versão 1.1 do JQuery e com a evolução no desenvolvimento e implementação de novas funcionalidades, foram encontrados diversos bugs e limitações na biblioteca para atender a todos os requisitos. Os técnicos em interface do time fizeram algumas análises e verificaram que migrando para a versão 1.3 do JQuery seriam sanadas 
a grande maioria dos bugs e seria possível implementar novas funcionalidades para aprimoramento do sistema.

CASO 2: Definição da forma de persistência de eventos de monitoração na base de dados.

O sistema de monitoração desenvolvido baseava-se no framework Nagios como plataforma de backend (http://www.nagios.org/). Porém, o Nagios tem limitações arquiteturais que limitavam a escalabilidade da ferramenta. Havia a necessidade de monitorar uma escala de milhares de dispositivos e nesses valores, não era possível utilizar somente um backend da ferramenta. Como forma de otimizar o desempenho do Nagios, optou-se por somente persistir as mudanças de status da monitoração e também não persistir as métricas coletadas nos agentes de monitoração.

CASO 3: Utilização do $M O N$ (https://mon.wiki.kernel.org/) como agente de monitoração na ferramenta.

"MON" é uma ferramenta que trabalha como um agente para a monitoração de disponibilidade de serviços e envio de alertas exclusivamente em servidores. Tomou-se a decisão de utilizar esse agente, pois, os principais entregáveis do projeto diziam respeito à monitoração de servidores e serviços ao qual o agente é compatível. É um componente pronto (oriundo do projeto OpenSource), de fácil manutenção e configuração e a curva de aprendizado para desenvolvimento de novos plug-ins de monitoração é pequena.

CASO 4: Ter o CMDB (Configuration Management Database) como mandatório na inserção de informações na ferramenta.

O desenvolvimento da ferramenta de monitoração e automação foi realizado paralelamente à implementação do ITIL (Information Technology Infrastructure Library) na empresa [Bon 2000]. Implementou-se uma ferramenta de gestão de problemas, mudanças e gestão de configuração (CMDB). A ferramenta de monitoração necessita de toda uma base de CI (Componentes de Configuração) e relacionamentos. Esses CI's são basicamente servidores, racks, switches, portas de switches, roteadores entre outros. A forma de se alimentar a ferramenta era exclusivamente via integração do CMDB.

\section{Resultados}

Em um primeiro momento com o auxílio de um profissional que participou do projeto, desde o seu início, identificou-se que a empresa, embora tenha noção do conceito de Dívida Técnica, não o considera na prática no momento de tomada de decisão estratégica e não o utiliza na gestão dos projetos. Logo, o passo seguinte foi a realização das entrevistas com cinco pessoas da equipe do projeto, das quais, apenas três estavam no projeto desde o início. Outras três pessoas consideradas chaves no momento de tomada de decisão, não participam mais do projeto e por isso, não foi possível realizar entrevistas com as mesmas. O resultado da análise dos dados e categorização dos mesmos será apresentado abaixo.

Após a conceitualização e caracterização de cada caso chegou-se ao diagrama abaixo que apresenta de forma generalista os conceitos e categorias em comum nos quatro casos estudados. 


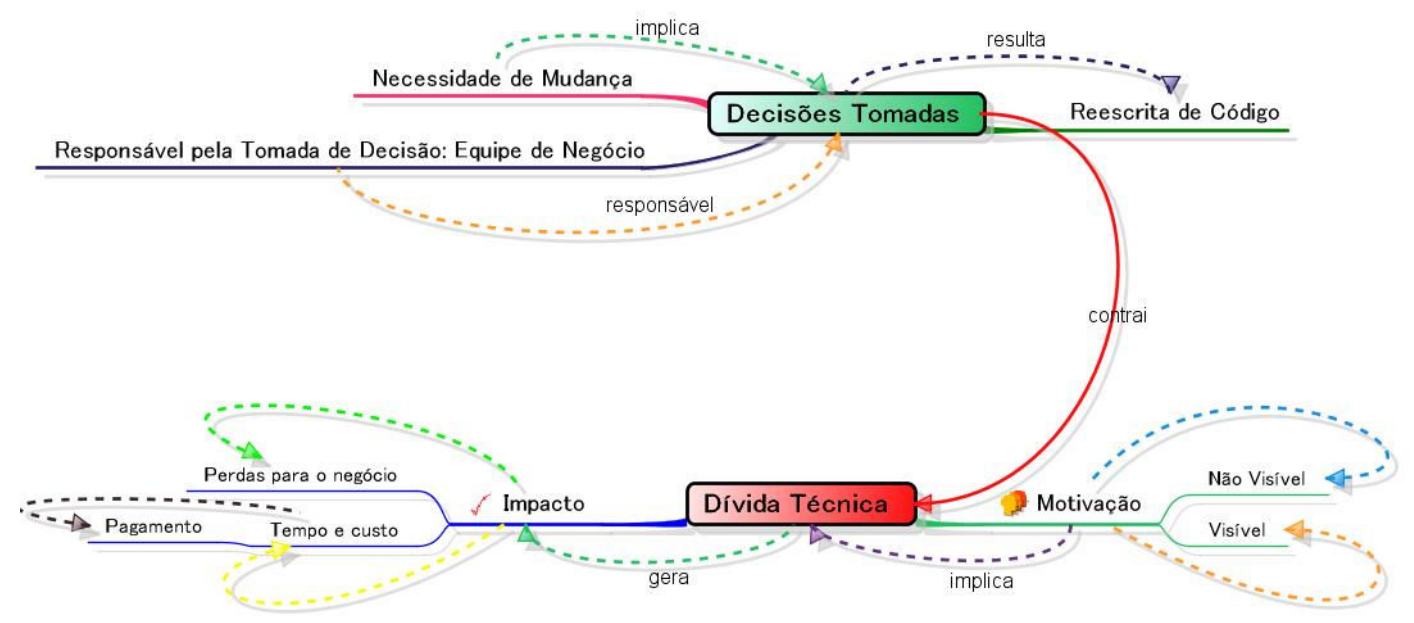

Figura 1 - Modelo resultante da caracterização e conceitualização dos casos.

Como se pode observar na Figura 1, todas as Dívidas estudadas foram originadas pelo surgimento de uma necessidade de mudança, seja ela oriunda por problemas de desempenho do sistema, ou por restrições do próprio negócio. Onde após essa necessidade de mudança, poucos estudos foram realizados para identificar qual seria a melhor solução. Comumente um membro da equipe com maior poder de arguição e/ou por dominar determinada tecnologia, convencia tomadores de decisão a seguir por determinado caminho. Esse processo implicou nas Dívidas Técnicas estudadas sem a percepção das pessoas envolvidas no projeto e que ao longo do tempo foram sofrendo impactos por isso, os primeiros impactos foram os atrasos na entrega como pode ser observado na Figura 2.

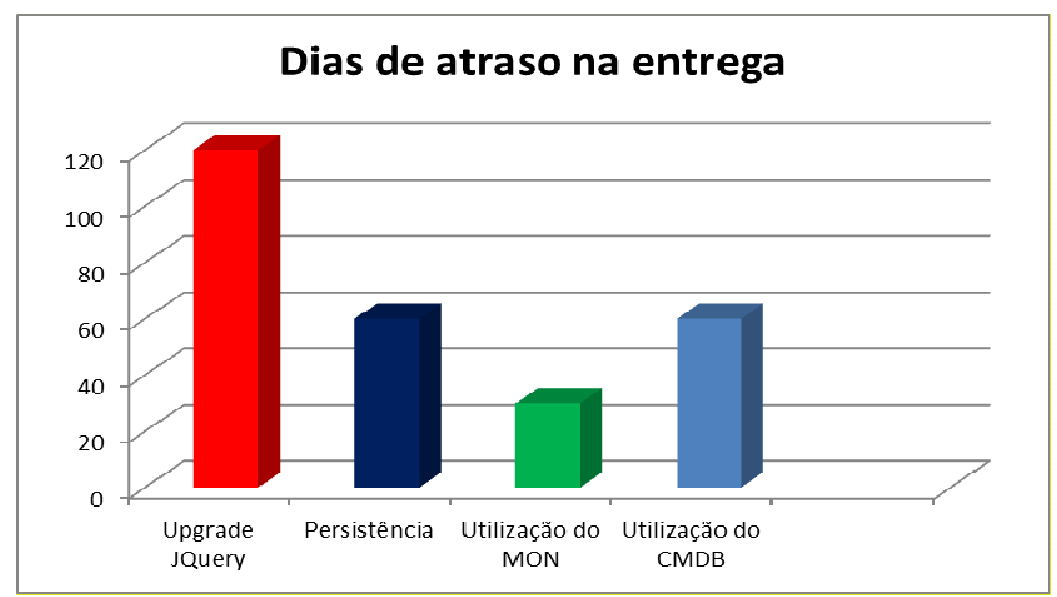

Figura 2 - Impacto em dias de atraso na entrega de cada caso.

Fica claro na Figura 2, que a entrega foi atrasada em muitos dias. Além disso, observou-se também, que impactos menos visíveis vêm sendo pagos até hoje na forma de reescrita de código e readequação de processo, onde muitas vezes o negócio precisa ser adaptado à ferramenta/tecnologia.

A partir do modelo criado na Figura 1, foi realizada a classificação de cada caso:

\section{Caso 1:}

Através das evidências coletadas, identificou-se que no momento da Tomada de Decisão do Caso 1 contraiu-se uma Dívida Técnica que ficou categorizada da seguinte forma: 
- Decisão Tomada: Fazer upgrade da Versão do JQuery.

- Influências dessa Decisão: Colaborador com grande conhecimento técnico convenceu a todos de que seria algo rápido de se fazer e o ganho seria grande.

- Motivação:

oVisivel

- Poderiam corrigir alguns bugs e ganhar em desempenho. ○Não Visível

-Técnicos convenceram que aquela era a melhor forma de fazer;

- Primeiro, fizeram uma análise baseada apenas no documento de benchmark divulgado no site da ferramenta.

-Segundo, resolveram mudar a arquitetura de todo o frontend do projeto, porém, não deixaram isso claro aos outros membros da equipe e para o cliente.

\section{- Impactos:}

oDireto:

-Um planejamento inicialmente estimado para ser executado em cerca de 2 meses, demorou mais de 6 meses.

-Equipe com alto grau de pressão, estresse e desgaste.

oIndireto:

- Cliente cobrando resultados.

- Muitas outras novas funcionalidades eram desenvolvidas e não podiam ser colocadas no ambiente de produção visto que ainda não se tinha um frontend funcional por causa do upgrade.

-Cliente final insatisfeito.

\section{Caso 2:}

Identificou-se que no momento da Tomada de Decisão, contraiu-se uma Dívida Técnica que ficou categorizada da seguinte forma:

- Decisão Tomada: Definição da forma de persistência de eventos de monitoração na base de dados.

- Influências dessa decisão: Membro da equipe aponta como melhor solução no momento, pois detinha conhecimento de tal tecnologia.

- Motivação:

oVisivel: Necessidade de mudança da forma de persistência de eventos de monitoração.

- O uso desta ferramenta atendia o único requisito solicitado no momento.

oNão Visível: Era de conhecimento do profissional que sugeriu usá-la e pouco ou nenhuma busca foi realizada para encontrar outra alternativa.

\section{- Impactos:}

oDireto: Tempo de entrega de novos componentes demoravam mais do que esperado, pois foi preciso se adaptar a tecnologia implantada.

oIndireto: A falta de um conhecimento mais detalhado do negócio, como: onde se pretendia chegar? Pode levar a equipe a cometer erros no momento da escolha de determinada tecnologia, implicando assim na necessidade de 
mudança da mesma em um futuro breve e/ou na limitação de possíveis soluções que podem ser oferecidas ao cliente.

\section{Caso 3:}

Identificou-se que no momento da Tomada de Decisão contraiu-se uma Dívida Técnica que ficou categorizada da seguinte forma:

- Decisão Tomada: Utilização do $M O N$ (https://mon.wiki.kernel.org/) como agente de monitoração na ferramenta.

- Influências dessa decisão: Cliente externo, coloca como restrição mandatória não utilizar outra alternativa cogitada, que no caso era SNMP (http://www.snmplink.org/).

- Motivação:

oVisivel

- Restrição do time de segurança. Provável falta de conhecimento oNão Visível técnico dos mesmos por impor esta limitação.

- Falta de questionamento e também provável conhecimento técnico para fazer valer tal alternativa por parte da equipe do projeto.

- Falta de análise mais profunda levando em conta o que produtos da concorrência já estavam disponibilizando no mercado.

\section{- Impactos:}

oDireto: Reescrita de código para que novas funcionalidades se adaptassem a solução existente, até chegar ao momento em que uma nova solução foi adquirida e adicionada a solução anterior.

oIndireto: Muitas estimativas sobre dados de infraestrutura foram perdidos.

\section{Caso 4:}

Identificou-se que no momento da Tomada de Decisão, contraiu-se uma Dívida Técnica que ficou categorizada da seguinte forma:

- Decisão Tomada: Ter o CMDB (Configuration Management Database) como mandatório na inserção de informações na ferramenta.

- Influências dessa decisão: Mandatória da diretoria da empresa.

- Motivação: Dados espalhados em vários lugares e muitas vezes inconsistentes.

- Impactos:

oDireto: Muitas funcionalidades ficaram atrasadas pois dependiam de dados advindos desse sistema e não estavam atualizadas.

oIndireto: Às vezes o que é apontado como um bug do sistema, na verdade é apenas inconsistência nos dados advindos do CMDB e dispende-se certo tempo até se chegar a esta conclusão e/ou provar isso para o relator do bug.

\section{Discussão dos resultados}

Em todos os casos aqui estudados pode-se dizer que as Dívidas Técnicas contraídas foram de curto prazo e inseridas de forma Não Intencional, como classificam as pesquisas de [McConnel 2007; McConnel 2008 e Fowler 2009]. 
Em relação ao estudo de Kaiser (2011), observou-se que a aderência dos desenvolvedores pode ser uma barreira para se pagar as Dívidas Técnicas, bem como o volume de negócios, onde novos requisitos surgem a todo instante.

As pesquisas de McConnel (2008) destacam que o primeiro passo a ser dado é aumentar a visibilidade da Dívida Técnica. Nos casos 1, 2 e 3 ficou claro que a Dívida Técnica foi contraída devido à falta de visibilidade da equipe sobre o real problema do negócio.

Mar (2010) ressalta que para saber se um sistema possui Dívida Técnica ou está em 'design death' ' é preciso prestar atenção em 3 indicadores: (1) se os componentes do sistema legado estão interligados de tal forma que não é possível isola-los; (2) quando não há nenhum indício de testes no código - sem testes unitários abrangentes é impossível refatorar o código para um estado mais gerenciável e (3) se o conhecimento do código do sistema está segmentado em apenas uma ou duas pessoas da empresa. Podemos observar que se olhássemos para o sistema analisando apenas esses três fatores, já identificaríamos que o mesmo possuía Dívida Técnica, pois, nos $\operatorname{Casos} 1,2$ e 3 a decisão foi tomada devido ao poder de convencimento de um membro da equipe com maior conhecimento técnico em determinada tecnologia, no caso 4 não se aplica essa classificação pois o mesmo foi oriundo de uma decisão externa mandatória. No Caso 3 os componentes do sistema estão completamente dependentes um do outro.

Nos estudos de Guo (2009) e Guo (2011) são propostos mecanismos para o gerenciamento da Dívida Técnica e sugerem que Dívida Técnica deve ser considerada como um tipo particular de risco em manutenção de software. Não se aplicam a este estudo, pois, ficou claro que o conceito de Dívida Técnica era desconhecido pela equipe de desenvolvimento, logo a mesma não foi gerida, apenas inserida no sistema. O mesmo vale para a pesquisa de Izurieta (2012) que propõe um modelo embasado nas abordagens existentes que visa facilitar a organização, visualização e identificação de Dívida Técnica.

Em um estudo recente publicado por [Allman 2012] destaca que Dívida Técnica é inevitável, onde o objetivo não deve ser eliminá-la, mas sim administrá-la. E comunicação, compreensão e gestão da Dívida Técnica podem fazer uma enorme diferença a longo e curto prazo para o sucesso do projeto. O estudo realizado enfatiza tal importância, deixando claro que se a Dívida Técnica for inevitável é preciso ter ciência dessa contração para que o seu impacto possa ser administrado e utilizado em benefício do projeto.

Seaman (2012) propõe formas de como tomar decisões considerando Dívida Técnica, uma vez que a Dívida Técnica já tenha sido identificada e medida. Como supracitado, nos casos estudados as Dívidas Técnicas foram ignoradas, não sendo possível utilizar tais abordagens sugeridas por Seaman. Porém, cabe ressaltar que o alto impacto gerado pela contração da Dívida Técnica, enfatiza a necessidade de considerá-la no momento de tomada de decisão como sugere Seaman.

Os casos também foram classificados seguindo as propriedades de Dívida Técnica: decisão, benefícios, dívida, juros e pagamento, criadas por Cunningham (1992), como pode ser observado na Figura 3.

\footnotetext{
${ }^{1}$ Quando um código possui tantas Dívidas Técnicas que o esforço necessário para alterar qualquer parte dele é muito oneroso, dizemos que o mesmo está com 'design morto'. - Informação retirada do artigo “Technical Debt and Design Death", Kane Mar e Michael James.
} 


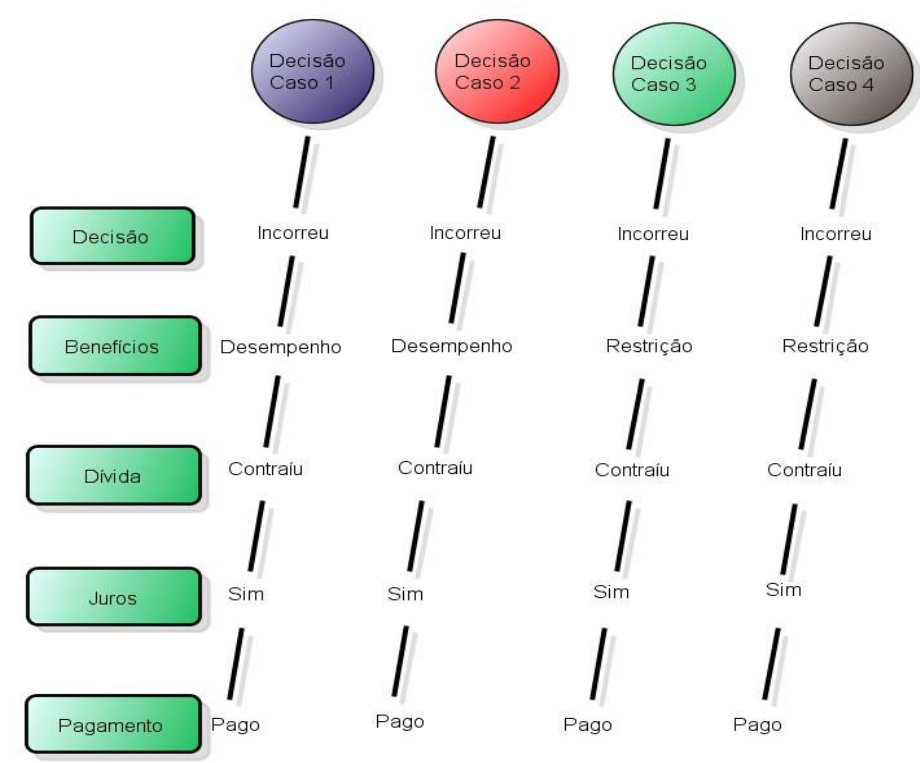

Figura 3 - Classificação dos casos segundo as propriedades criadas por Cunningham (1992).

Na Figura 3 é possível identificar que as decisões tomadas incorreram em Dívida Técnica em prol de alguns benefícios e que as mesmas foram pagas ao longo do tempo a custos altos como: clientes insatisfeitos, concorrência oferecendo soluções mais completas no mercado; altos custos para fazer refatoração, negócio tendo que ser adaptado a tecnologia, pois a mesma não atendia às necessidades, equipe com alto grau de estresse e sem motivação para dar continuidade ao projeto.

Por fim, este estudo não só corrobora a importância de se considerar Dívida Técnica como deixa claro que Dívidas não geridas se transformam em grandes problemas que podem perdurar por toda a vida útil do sistema. E que quanto mais o tempo passa maior o custo que se paga por ignorá-las. Destaca também a importância de se haver comunicação clara entre membros de equipe e também entre equipe técnica e área de negócio. Pois, se a equipe não sabe aonde se pretende chegar com o negócio, determinada solução pode sim atender aquele requisito em dado momento, mas se tornar em uma grande Dívida no momento seguinte.

Acredita-se que foi dado um importante passo, identificando, monitorando e caracterizando as Dívidas Técnicas e a partir disso criando um modelo que de forma generalista mostra onde elas surgem, quais suas influências, motivações e para onde tem seguido. Assim hoje é possível passar a monitorá-las e ir pagando as mesmas ou frações das mesmas ao longo do tempo de forma estratégica ou até mesmo é possível se chegar ao entendimento de que é melhor não pagá-las. Bem como este exemplo pode ser replicado em outros projetos em andamento buscando outras Dívidas Técnicas que podem estar causando impactos negativos no negócio. Este mesmo conceito pode ser replicado para outros projetos que não somente de tecnologia. E as motivações e influências de inserção de Dívida aqui encontradas podem ser consideradas em novos projetos no momento de tomada de decisão para que os mesmos erros e consequentes impactos não se repitam.

\section{Ameaças a Validade}

Os dados da pesquisa foram coletados através de entrevistas, logo, para uma versão futura seria interessante realizar triangulação de dados buscando confrontar as evidências com 
dados de documentos do projeto. Não foi possível fazer um cálculo detalhado sobre custo, pois, não foi possível ter acesso a variáveis como: valor homem/hora, custo da manutenção do sistema enquanto a nova versão não era disponibilizada e impacto/perdas para o negócio pelo atraso das entregas. Por isso, o gráfico de impacto, que pode ser visualizado na Figura 2 possui apenas os dias de atraso da entrega do projeto.

\section{Trabalhos Futuros}

Objetiva-se dar continuidade a esta pesquisa, replicando o estudo para outros projetos da empresa, bem como buscar acesso a documentos para corroborar as evidências encontradas.

Em relação à Dívida Técnica, muitas ainda são as lacunas existentes em como identificar, gerir e utilizá-la de forma estratégica ao longo do projeto. Cabe ressaltar que até o momento as pesquisas realizadas vêm sendo embasadas na metáfora criada por Cunningham (1992), pois não existe uma teoria formada sobre Dívida Técnica.

\section{Agradecimentos}

Agradecemos em especial à empresa que nos propiciou acesso a seu ambiente, dados e colaboradores. E a todos os colaboradores que prontamente dispuseram seu tempo para contribuir com a pesquisa. Agradecemos também a Fapesp agência financiadora dos projetos número; 2011/23470-7 e 2009/16354-0.

\section{Referências}

Allman, E. (2012) "Managing Technical Debt". Publications of the ACM, Volume 10, Issue 3, Março, 2012.

Bon, V.J., et.al (2000) "Introduction to ITIL". The Stationery Office. U.K.ISBN 0113309732.

Brown, N. et al. (2010). Managing technical debt in software-reliant systems. The FSE/SDP workshop on Future of software engineering research. New Mexico: [s.n.]. p. 47-52.

Cunningham, W. (1992) "The WyCash Portfolio Management System". Conference on Object-Oriented Programming Systems, Languages, and Applications (OOPSLA'92). Vancouver: [s.n.].

Fowler, M. (2009) “Technical Debt Quadrant.” Disponível em $<$ http://www.martinfowler.com/bliki/TechnicalDebtQuadrant.html $>$. Acesso em: 26 maio. 2012, 10:37.

Gartner. (2012). "Gartner Estimates Global 'IT Debt' to Be $\$ 500$ Billion This Year, with Potential to Grow to $\$ 1$ Trillion by 2015." http://www.gartner.com/it/page.jsp?id=1439513 . Último acesso, em abril/2012.

Gomes, R. (2011) “Caracterização e Conceituação Teórica da Metáfora de Débito Técnico Através de um Estudo Exploratório." Dissertação de Mestrado Universidade Federal de Pernambuco, Setembro, 2011. 
Gomes, R. Cavalcanti, A. Tonin, G. et.al. (2011) "An Extraction Method to Collect Data on Defects and Effort Evolution in a Constantly Modified System." MTD '11 Proceedings of the 2nd Workshop on Managing Technical Debt. ACM, New York, USA, 2011.

Guo, Y. (2009) "Measuring and Monitoring Technical Debt." 4th International Doctoral Symposium on Empirical Software Engineering. [S.1.]: [s.n.]. p. 25-46.

Guo, Y. and Seaman, C. (2011) "A portfolio approach to technical debt management." Proceeding of the 2nd working on Managing technical debt. [S.1.]: [s.n.].

Guo, Y, Seaman, C. Gomes, R. Cavalcanti, A. Tonin, G. et.al (2012) “Tracking Technical Debt - An Exploratory Case Study". Proceedings of the 27th IEEE International Conference on Software Maintenance (ICSM' 11), 528-531, 2011.

Highsmith, J. (2011). "Accelerating Enterprise Agility Adaptive Leadership." Agile Conference 2011.Salt Lake City, Utah, Agosto.

Izurieta, C. Vetro, A. Zazworka, N. Cai, Y. Seaman, C and Shull, F. (2012) "Organizing the Technical Debt Landscape." Third International Workshop on Managing Technical Debt in conjunction with ICSE 2012.

Mar, K e James, M. (2010) “The Technical Debt and Design Death.” CollabNet.

McConnel, S. (2008) "Managing Technical Debt." Construx Software, Version 1, 2008. Disponível em <http://www.construx.com/File.ashx?cid=2797>. Acesso em: 26 maio.2012, 10:28.

McConnel, S. (2007) “Technical Debt.” Construx Software,Version 1. Disponível em

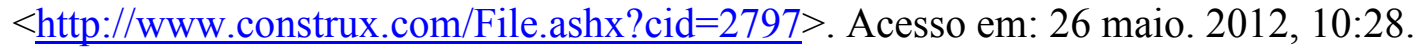

Merriam, S. B. (2009) "Qualitative Research: A Guide to Design and Implementation." 3. ed. [S.1.]: Jossey-Bass.

Robson, C. (2002) "Real World Research.” Blackwell, (2nd edition).

Seaman, C., et.al. (2012) "Using Technical Debt Data in Decision Making: Potential Decision Approaches." MTD2012: 3rd Workshop on Managing Technical Debt, at ICSE 2012, Zurich, Switzerland.

Strauss, A. and Corbin, J. (2008) "Pesquisa qualitativa: Técnicas e procedimentos para o desenvolvimento de teoria fundamentada nos dados." Tradução de Luciane de Oliveira da Rocha. 2. ed. Porto Alegre: Artmed.

Tonin, G.S (2011). "Decisões em Projetos de Desenvolvimento de Software e a Metáfora do Débito Técnico: Um Estudo De Caso Exploratório". Dissertação de Mestrado Universidade Federal de Pernambuco, Setembro, 2011.

VersionOne. (2011). "State of Agile Development Survey Results" http://www.versionone.com/state of agile development survey/11/. Último acesso, $\mathrm{abri1} / 2012$.

Williams, L. (2010) "Agile Software Development Methodologies and Practices". Advances in Computers, vol. 80, pp. 1-44.Yin, R. K. (2003) "Case Study Research: Design and Methods.” 3. ed. [S.1.]: Sage. 
Appendix E

First Interview applied in Extreme Programming Laboratory, 2013 Edition 


\section{Roteiro de Entrevista Experimento Laboratório XP}

Este roteiro tem como objetivo guiar a entrevista com as equipes dos projetos da disciplina Laboratório de Programação Extrema.

\section{Primeiros Passos}

- Pesquisador se apresenta.

- Pesquisador solicita permissão para usar 30 min do tempo do respondente.

- Pesquisador informa que entrevista será gravada.

- Pesquisador apresenta sua pesquisa e deixa claro o objetivo da entrevista.

- Se todos estão de acordo pesquisador inicia entrevista.

\section{Objetivo}

Identificar a influencia de deixar explicita Dívida Técnica para o time e investigar quais os efeitos causados no time.

\section{Questões abordadas}

\section{// verificar se estava o coach no par}

1) O fato de estar consciente sobre Dívida Técnica afetou de alguma forma como vocês desenvolvem software? Como?

//afetou a forma como você desenvolve software? A forma como você codifica? Como //você pensa?

//fazer o quadro teve overhead...

//no planejamento ter que olhar para a lista de dívidas foi melhor? Ou apenas gerou //mais trabalho? Trouxe algum benefício real? Caso sim qual?

2) Mudou a forma como vocês planejam as atividades do desenvolvimento do software?

3) Mudou a forma como vocês codificam?

4) Mudou a forma de fazer teste?

5) Afetou de alguma forma a negociação com cliente?

6) Alguma parte do processo mudou por vocês evidenciarem Dívida Técnica?

7) Em algum momento vocês olham para essas Dívidas Técnicas identificadas? //Em qual momento? //O que fazem

8) Ouve alguma parte do processo que passou a demorar mais tempo porque você estava olhando para Dívida Técnica?

9) Alguma parte do processo (desenvolvimento/planejamento) ficou mais fácil por causa disso? 
10) De todo o processo de tornar Dívida Técnica visível qual a parte mais difícil?

11) Qual parte do modelo/ficha foi mais difícil deixar evidente?

//Porque?

12) Qual (s) dado (s) do modelo vocês consideram mais importante coletar?

13) Você adicionaria alguma informação na coleta de dados sobre Dívida Técnica?

14) Na sua visão quais os pontos positivos de manter visível a Dívida Técnica?

15) Na sua visão quais os pontos negativos de manter visível a Dívida Técnica?

16) Você acredita que é importante identificar Dívida Técnica?

17) Por que você acredita que é/não é importante identificar Dívida Técnica?

18) Vocês pagaram as Dívidas Técnicas?

19) Por que vocês pagaram/não as Dívidas Técnicas?

20) Você acredita que Dívida Técnica impacta a qualidade do software?

- Pesquisador agradece aos entrevistados. 
Appendix F

First Questionnaire Applied in Extreme Programming Laboratory, 2014 Edition 


\section{Dívida Técnica Lab XP}

Este questionário tem como objetivo contribuir para uma pesquisa de doutorado voltada para a área de qualidade de software. Desde já agradecemos seu empenho e dedicação.

Obrigada! :)

*Obrigatório

1. 1.0 Sua equipe se preocupa com qualidade? *

2. 1.1Caso sim o que vocês fazem voltado a qualidade? *

3. 1.2 Vocês utilizam ferramentas de controle de qualidade? Caso sim quais?

4. 1.2.1 Caso utilizem alguma ferramenta, os itens apontados por estas ferramentas são relevantes? Porque? *

5. 2.0 Você conhece o termo Dívida Técnica? *

6. 2.0.1 Os controles de qualidade utilizados por vocês indicam dívidas técnicas? *

7. 3.0 Vocês tem considerado os relatórios resultantes da ferramenta SONAR/Code Climate? *

8. 3.0.1 Caso sim, quais? * 
9. 3.2 Vocês tem considerado o item Dívida Técnica? Sim ou Não e Porque? *

10. 3.2.1 Vocês veem alguma relevância neste item? *

11. 3.4 Ter as dívidas técnicas evidênciadas fez com que vocês tomassem alguma atitude diferente? *

12. 3.4.1 Caso sim, qual? *

13. 3.4.2 Por elas estarem evidenciadas existiu algum esforçou para pagá-las? *

14. 3.4.4 Elas estarem evidênciadas influenciou em algo na equipe? *

15. 3.4.5 Em algum momento estas listas de possíveis dívidas técnicas foram consideradas? *

16. 3.4.6 O esforço para o pagamento das dívidas foi indiferente de tê-las ou não evidenciadas.

Marcar apenas uma oval.

$\operatorname{Sim}$

Não

Não sei 
Appendix G

Second Questionnaire Applied in Extreme Programming Laboratory, 2014 Edition 


\section{Dívida Técnica}

*Obrigatório

1. 1.0 Sua equipe se preocupa com qualidade? *

2. 1.1 Caso sim o que vocês fazem voltado a qualidade? *

3. 1.2 Vocês utilizam ferramentas de controle de qualidade? Caso sim quais?

4. 1.2.1 Caso utilizem alguma ferramenta, os itens apontados por estas ferramentas são relevantes? Porque? *

5. 2.0 Após o bate papo sobre Dívida Técnica, você passou a entender o que é Dívida Técnica? *

6. 2.0.1 Os controles de qualidade utilizados por vocês indicam dívidas técnicas? *

7. 3.0 Vocês tem considerado os relatórios resultantes da ferramenta SONAR/Code Climate? *

8. 3.0.1 Caso sim, quais? *

9. 3.2 Vocês tem considerado o item Dívida Técnica? Sim ou Não e Porque? * 
10. 3.2.1 Vocês veem alguma relevância neste item? *

11. 3.4 Ter as dívidas técnicas evidênciadas fez com que vocês tomassem alguma atitude diferente? *

12. 3.4.1 Caso sim, qual? *

13. 3.4.2 Por elas estarem evidenciadas existiu algum esforçou para pagá-las? *

14. 3.4.4 Elas estarem evidênciadas influenciou em algo na equipe? *

15. 3.4.5 Em algum momento estas listas de possíveis dívidas técnicas foram consideradas? *

16. 3.4.6 O esforço para o pagamento das dívidas foi indiferente de tê-las ou não evidenciadas.

Marcar apenas uma oval.

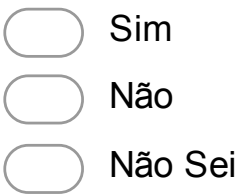

17. 3.5 Nos próximos projetos que irão atuar pretendem considerar o termo Dívida Técnica? Explique.

18. 3.6 Vocês consideravam os itens de dívida técnica evidenciados pelas ferramentas? *

19. 3.7 Vocês consideraram alguma dívida técnica que não foi apontada pelas ferramentas? * 
20. 3.7.1 Caso sim, qual (is)? *

21. 3.7.2 Que dívidas foram consideradas?

Um exemplo? *

Powered by

国 Google Forms 
Appendix $\mathrm{H}$

Questionnaire applied in the Company A 


\section{Pesquisa de avaliação sobre identificação e gererenciamento da dívida técnica no Jira}

Essas perguntas tem o objetivo de ter uma primeira avaliaçào para que consiga finalizar a minha dissertação de mestrado. Conto com a sua colaboraçăo no preenchimento. Obrigado.

* Required

1. A identificação e gerenciamento da divida técnica tem algum efeito na qualidade do software? 'Responda Sim ou Não e justifique

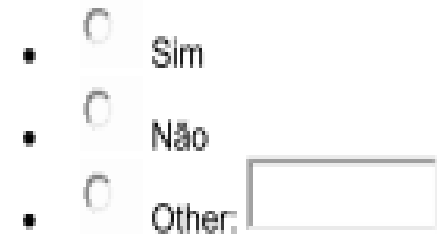

Justifique a sua resposta *

2. Quais os efeitos para o desenvolvimento do produto ao se identificar e gerenciar a divida técnica? * 
3- Ao se explicitar a dívida técnica, o que mudou no gerenciamento do backlog do produto? *

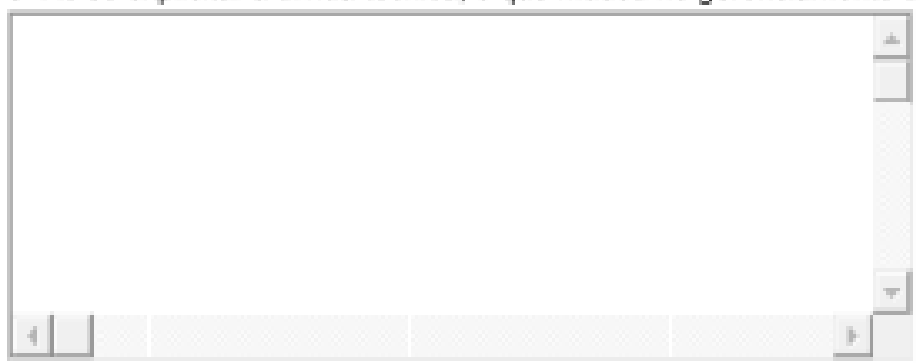

4- Poderia elencar pontos positivos e negativos do gerenciamento de divida técnica através do Jira? *

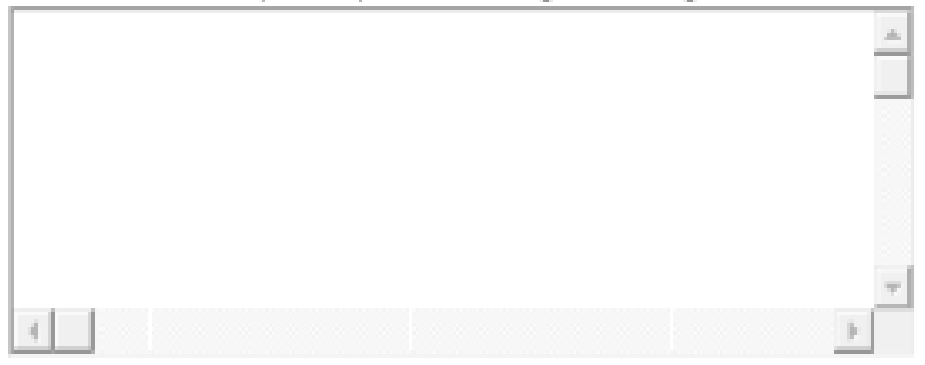

5- Qual o impacto do modelo de gerenciamento de divida técnica no processo de desenvolvimento do time?*

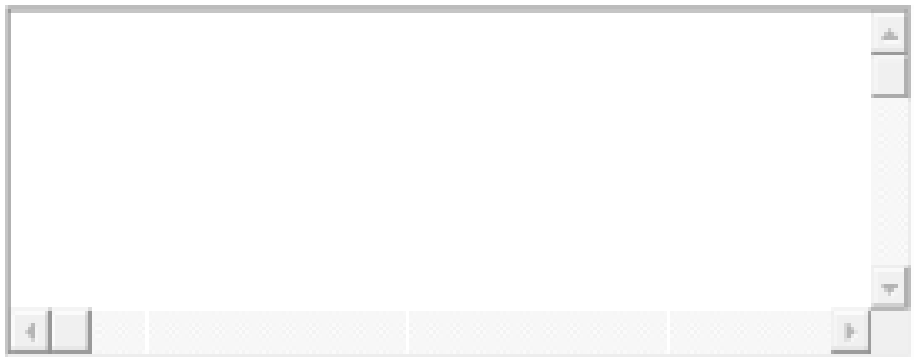

6- Qual o impacto do modelo de gerenciamento criado, na qualidade do software desenvolvido? *

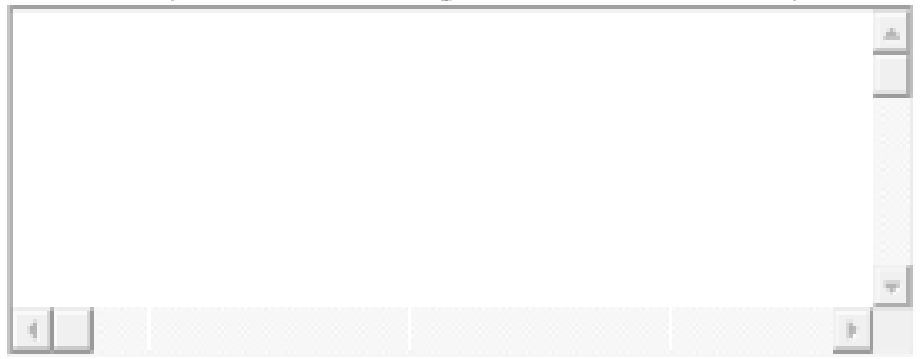


7- O que e dívida técnica para vocé? "Descreva de forma clara e simples o seu entendimento sobre divida técnica?

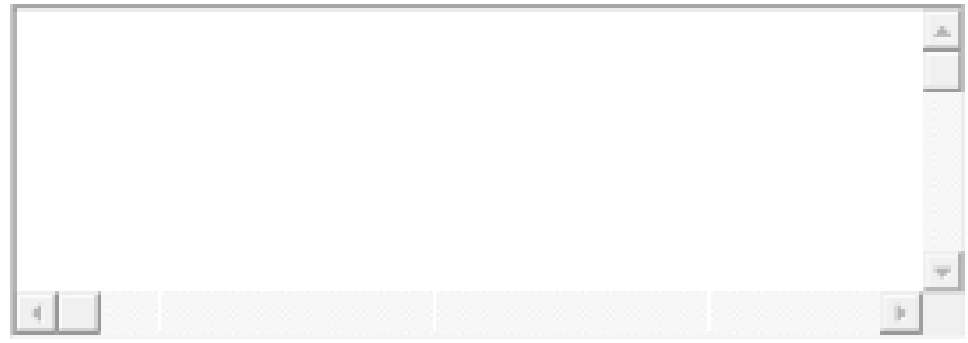

Qual time de desenvolvimento voce faz parte *

- $r$ Quebec

- $r$ Plataforma de dominios

- $\mathrm{C}$ Plataforma de Host

Back submt

Never submit passwords through Google Forms.

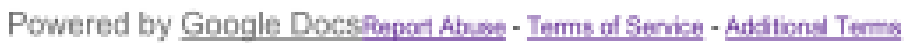




\section{Bibliography}

[1] Allman, E. Managing technical debt. Cutter IT Journal - The Journal of Information Technology Management. 5, 10 (Oct 2012), 50-55.

[2] Alves, N.S.R, M. T. D. M. M. S. R. F. S. S. C. Identification and management of technical debt: A systematic mapping study. vol. 70, Journal of Information and Software Technology, pp. $100-121$.

[3] Arquigrafia. Arquigrafia project. Online at http://www.arquigrafia.org.br/, May 2016.

[4] Avgeriou, P. Kruchten, P. O. I. S. C. Managing technical debt in software engineering. vol. 6, Dagstuhl Reports, pp. 110-138.

[5] Baskerville, R. Investigating information systems with action research. Communications of AIS. 2, 19 (1999).

[6] BAvani, R. Distributed agile, agile testing, and technical debt. vol. 29, IEEE Software,, pp. 28-33.

[7] Beck, K. Extreme Programming Explained: Embrace Change. Person Education Inc, 2005.

[8] Bellomo, S. Nord, R. O. I. P. M. Got technical debt: surfacing elusive technical debt in issue trackers. MSR '16 Proceedings of the 13th International Conference on Mining Software Repositories, pp. 327-338.

[9] Besker, T. Martini, A. B. J. Managing architectural technical debt: A unified model and systematic literature review. vol. 135, Journal of Systems and Software, pp. 1-16.

[10] Bon, V. E. Introduction to ITIL. The Stationery Office, 2000.

[11] Brown, N., N. R. O. I. K. P. L. E. Hard choice: A game for balancing strategy for agility. In Software Engineering Education and Training (CSEET) - Software Engineering Education and Training (CSEET)., 2011 24th IEEE-CS Conference on, Hon-olulu, HI, USA (2011).

[12] Brown, N., Y. Y. G. Y. K. R. K. M. K. P. L. E. M. A. N. R. O. Y. S. R. C. S. K. Z. N. Managing technical debt in software-reliant systems, proceedings of the fse/sdp workshop on future of software engineering research. ACM, pp. 47-52.

[13] Buschmann, F. To pay or not to pay technical debt. vol. 28, IEEE Software, pp. 29-31.

[14] Chicote, M. Startups and technical debt managing technical debt with visual thinking. IEEE/ACM 1st International Workshop on Software Engineering for Startups (SoftStart).

[15] Codabux, Z. Williams, B. Managing technical debt: an industrial case study. Proceedings of the 4th International Workshop on Managing Technical Debt (MTD'13), IEEE, pp. 8-15.

[16] Codabux, Z. Williams, B. N. N. A quality assurance approach to technical debt. Proceedings of Software Engineering Research and Practice - SERP.

[17] CoGro. Cogroo project. Online at http://ccsl.ime.usp.br/cogroo/, May 2016. 
[18] Conte, T. Cabra, R. T. G. Applying grounded theory in a qualitative analysis in a observation study about software engineering.

[19] Corbin, J. Strauss, A. Grounded theory research: Procedures, canons and evaluative criteria. Zeitschrift fur Soziologie. 1990, 6 (Dec 2001), 3-30.

[20] Cunningham, W. Experience report-the why cash portfolio management system.

[21] CurTis, B. Paying down the interest on your applications a guide to measuring and managing technical debt.

[22] Curtis, B. Sappidi, J. S. A. Estimating the size,cost, and types of technical debt. Proceedings of the 3rd International Workshop on Managing Technical Debt - MTD, IEEE, pp. 49-53.

[23] De Assunção, T.R. Rodrigues, I. V. E. F. R. D. S. T. Technical debt management in the brazilian federal administration. 6th Brazilian Workshop on Agile Methods.

[24] Dos Santos, P.S. Varella, A. D. C. B. D. Visualizing and managing technical debt in agile development: An experience report. vol. LNBIP 149, XP, Springer-Verlag Berlin Heidelberg, p. 121-134.

[25] Easterbrook, S. Singer, J. S. M. D. Selecting empirical methods for software engineering research. International conference on Automated Software Engineering. (2007).

[26] Einsenberg, R. A threshold based approach to technical debt. International conference on Automated Software Engineering. 37, 2 (March 2012), 1-6.

[27] Ernst, A.N, B. S. O. I. N. R. G. I. Measure it? manage it? ignore it? software practitioners and technical debt. ESEC/FSE 2015 Proceedings of the 2015 10th Joint Meeting on Foundations of Software Engineering, ACM, pp. 50-60.

[28] ERnst, N. On the role of requirements in understanding and managing technical debt. MTD.

[29] Falessi, D. Voegele, A. Validating and prioritizing quality rules for managing technical debt: An industrial case study. MTD, Bremen, Germany, IEEE.

[30] Falessi, D. Shaw, M. S. F. M. K. S. M. Practical considerations, challenges, and requirements of tool-support for managing technical debt. Proceedings of the 4th International Workshop on Managing Technical Debt (MTD'13), IEEE,, pp. 16-19.

[31] Fernandez-Sanchez, C., G. J. V. C., And Yague, A. An analysis of techniques and methods for technical debt management: a reflection from the architecture perspective. IEEE.

[32] Fernandez-SÁnchez, C. Humanes, H. G. J. D. J. An open tool for assisting in technical management. 43rd Euromicro Conference on Software Engineering and Advanced Applications.

[33] Flyvbjerg, B. Five misunderstandings about case-study research. Qualitative Inquiry. 12, 2 (Apr 2006).

[34] Fowlen, M. Technical debt. Online at https://martinfowler.com/bliki/ContinuousDelivery. html, May 2009.

[35] Fowler, M. Technical debt quadrant. Online at http://www.martinfowler.com/bliki/ TechnicalDebtQuadrant.html, May 2009.

[36] Fraser, S. Bishop, J. B. B. K. P. K. P. O. I. S. A. Technical debt: Past, present, and future. Proceedings of the 2013 International Conference on Software Engineering. (2013), 861-862. 
[37] Gartner. Gartner estimates global 'it debt' to be $\$ 500$ billion this year, with potential to grow to $\$ 1$ trillion by 2015 . Online at http://www.gartner.com/it/page.jsp?id=1439513, 2012. Accessed 30 Jun 2012.

[38] Glaser, B. Strauss, A. The discovery of grounded theory: Strategies for qualitative research. Aldine Transaction. (jan 1967).

[39] Goldman, A. Kon, F. S. P. Y. J. Being extreme in the classroom: Experiences teaching xp. Journal of the Brazilian Computer Society. 10, 2 (2004), 5-21.

[40] Gomes, R. Characterization and theoretical conceptualization of the technical debt metaphor through an exploratory study. Federal University of Pernambuco., 2 (2011).

[41] Guo, Y., and Seaman, C. Measuring and monitoring technical debt. IV International Doctoral Symposium on Software Engineering. Florida, USA.

[42] Guo, Y., And Seaman, C. A portfolio approach to technical debt management - mtd. 2nd International Workshop on Managing Technical Debt.

[43] Harun, F.M. Lichter, H. Towards a technical debt management framework based on costbenefit analysis. 10th International Conference on Software Engineering Advances (ICSEA 2015 , pp. $15-20$.

[44] Highsmith, J. The financial implicatitons of technical debt., 2010. Ú.

[45] Highsmith, J. Accelerating enterprise agility adaptive leadership. In Agile Conference 2011. Salt Lake City, Utah, USA. (2011).

[46] Holvitie, J. Software implementation knowledge management with technical debt and network analysis. IEEE.

[47] Holvittie, J., And Leppanew, V. Debtflag: Technical debt management with a development environment integrated tool. MTD, San Francisco, CA, USA, IEEE.

[48] Izurieta, C. Rojas.G. Griffith, I. Preemptive management of model driven technical debt for improving software quality. Quality of Software Architectures (QoSA), 11th International ACM SIGSOFT Conference on.

[49] Izurieta, C. Ozkaya, I. S. C. S. W. Perspectives on managing technical debt a transition point and roadmap from dagstuhl. Seminar on Technical Debt - Dagstuhl.

[50] Kitchenham, B.A. Dyba, T. J. M. Evidence-based software engineering. Proceedings of the 26th International Conference on Software Engineering - ICSE). (2004).

[51] Kruchten, P. Nord, R. O. I. Technical debt: From metaphor to theory and practice. vol. 29, IEEE Software, pp. 18-21.

[52] Kruchten, P. Nord, R. O. I. F. D. Technical debt:towards a crisper definition report. No. 51-54, 4th International Workshop on Managing Technical Debt, SIGSOFT Software Engineering.

[53] Lehman, M. On understanding laws, evolution and conservation in the large program life cycle. 213-221.

[54] Letouzey, J. The sqale method for evaluating technical debt. 2012 Third International Workshop on Managing Technical Debt (MTD), pp. 31-36.

[55] Li, Z. Avgerious, P. L. P. A systematic mapping study on technical debt and its management. vol. 101, The Journal of Systems and Software, Science Direct. 
[56] LI, J. Tor, S. K. J. R. C. Cost drivers of software corrective maintenance: An empirical study in two companies.

[57] Lim, E. TAKSAnde, N. S. C. A balancing act: what software practitioners have to say about technical debt. $22-27$.

[58] M., B. R. M. Special issue on action research in information systems: Making is research relevant to practice. MIS Quarterly 28, 3 (Set 2004).

[59] Martini, A. Bosch, J. C. M. Architecture technical debt: Understanding causes and a qualitative model. 40th Euromicro Conference on Software Engineering and Advanced Applications.

[60] MCConnelt, S. Managing technical debt. Online at http://www.construx.com/File.ashx? cid=2797, Nov 2008 .

[61] McConnell, S. Managing technical debt (slides)., Nov 2009.

[62] Mens, T. Demeyer, S. Software evolution.

[63] Merriam, S. Introduction to qualitative research.

[64] Merriam, S. Qualitative research. a guide to design and implementation.

[65] Mezuro. Mezuro project. Online at http://mezuro.org/pt, May 2016.

[66] Monitoria. Monitoria project. Online at www.monitoria.ime.usp.br, May 2016.

[67] Nord, L. OzkAyA, I. K. P. G. M. In search of a metric for managing architectural technical debt. Proceedings of the 10th Working IEEE/IFIP Conference on Software Architecture (WICSA'12),IEEE,.

[68] Nugroho, A.J. Visser, J. K. T. An empirical model of technical debt and interest. MTD, pp. 22-28.

[69] Oliveira, R. Goldman, A. M. C. Designing and managing agile informative workspaces: Discovering and exploring patterns. 4790-4799.

[70] Oliveira, F. Goldman, A. S. V. Managing technical debt in software projects using scrum: An action research. Agile Conference.

[71] Oliveira, R.G. Queda, F. Characterization and theoretical conceptualization of the technical debt metaphor through an exploratory case study. Master's thesis, 2011.

[72] Pires, R. Tonin, G. O. R. G. A. The effects of a model for identifying and managing technical debt in scrum project. 4790-4799.

[73] Pires.R. A systematic study on identifying and managing technical debt in a scrum development technology company. Master's thesis, 2014.

[74] Projects., X. L. Extreme programming projects - ccsl. Online at http://www.ccsl.org.br/ oldwiki/index.php, May 2016.

[75] Ramasubbu, N. Kemerer, C. Integrating technical debt management and software quality management processes: A normative framework and field tests. IEEE TRANSACTIONS ON SOFTWARE ENGINEERING.

[76] Ramasubbu, N. Kemerer, C. W. J. Managing technical debt: Insights from recent empirical evidence. vol. 32, IEEE Software, pp. 22-25. 
[77] Ries, E. Embrace technical debt. in: Lessons learned, available. Online at http://www. startuplessonslearned.com/2009/07/embrace-technical-debt.html, 2009. Accessed 04 Nov 2014.

[78] Robson, C. Real world research. a resource for social scientists and practitioner-researchers.

[79] Rubin, K. Essential Scrum. A Practical Guide To The Most Popular Agile Process. AddisonWesley, 2013.

[80] Runeson, M. Guidelines for conducting and reporting case study research in software engineering.

[81] Sandberg, A. Staron, M. A. V. Towards proactive management of technical debt by software metrics. 14th Symposium on Programming Languages and Software Tools, pp. 1-15.

[82] Santos, V. Goldman, A. S. C. Uncovering steady advances for an extreme programming course. 1.

[83] Schimd, K. A formal approach to technical debt decision making. 1.

[84] Schmid, K. On the limits of the technical debt metaphor: Some guidance on going beyond. In Fourth Workshop on Managing Technical Debt, Workshop at the International Conference on Software Engineering. (2013), IEEE, p. 4.

[85] Schwaber, K. Beedle, M. Agile software development with scrum.

[86] Seaman, C. Qualitative methods in empirical studies of software engineering. 557-572.

[87] Seaman, C., And Guo, Y. A model to technical debt management and identification. Accessed 08 Feb 2014.

[88] Seaman, C. Guo, Y. I. C. Y. Z. N. S. F. V. A. Using technical debt data in decision making: Potential decision approaches.

[89] Siebra, C.A. Tonin, G. D. O. R. C. A. M. R. S. A. Managing technical debt in practice: an industrial report. Proceedings of the 6th ACM - IEEE International Symposium on Empirical Software Engineering and Measurement - ESEM, pp. 247-250.

[90] Smith, R. Computer system design. vol. 13, Journal of GXP Compliance, pp. 44-48.

[91] Sommerville, I. Software engineering, 6th edition., 2000.

[92] SPORT. System specialist in sport. Online at http://journals.plos.org/plosone/article?id=10. 1371/journal.pone.0145733, May 2016.

[93] Spínola, R. Zazworka, N. V. A. S. C. S. F. Investigating technical debt folklore shedding some light on technical debt opinion.

[94] Stake, R. The art of case study research. Sage, 1995.

[95] Sterling, C. Managing Software Debt: Building for Inevitable Change. Addison-Wesley Professional, 2010, 2010.

[96] Strauss, A. Qualitative analysis for social scientists. New York, Cambridge University Press, 1987.

[97] Strauss, A. Corbin, J. Basics of Qualitative Research: Techniques and Procedures for Developing Grounded Theory., 2 ed ed. London, SAGE Publications, 1998. 
[98] Tamburri, D.A., K. P. L. P., And van Vliet, H. What is social debt in software engineering? CHASE, IEEE.

[99] Tassey, G. The economic impacts of inadequate infrastructure for software testing final report.

[100] Theodoropoulos, T. Hofberg, M. K. D. Technical debt from the stakeholder perspective. Proceedings of the 2nd International Workshop on Managing Technical Debt (MTD'11), ACM, pp. 43-46.

[101] Tom, E. Aurum, A. V. R. An exploration of technical debt. 1498-1516.

[102] Tonin, G. Decisions in software development projects and the technical debt metaphor: An exploratory case study. Master's thesis, 2011.

[103] Tonin, G.S. Chaves, R. G. A. S. V. A technical debt analysis in a technology company with the development based in scrum. Best paper.

[104] Tonin, G.S. Suiyama, V. S. N. S. D. B. D. P. D. S. V. G. A. Technical debt management: Where to start from? preliminary experiences from an action research study.

[105] Vathsavayi, S.H. Systã, K. Technical debt management with genetic algorithms. 42th Euromicro Conference on Software Engineering and Advanced Applications.

[106] VidaGeEK. Vidageek project. Online at http://aprenda.vidageek.net/, May 2016.

[107] Yin, R. Case study research: Design and methods. 1-44.

[108] Yli-Huumo, J. Maglyas, A. S. K. The sources and approaches to management of technical debt: A case study of two product lines in a middle-size finnish software company. Springer International Publishing Switzerland.

[109] Yli-Huumo, J. Maglyas, A. S. K. How do software development teams manage technical debt? - an empirical study. vol. 120, Journal of Systems and Software, pp. 195-218.

[110] Zazworka, N. Vetro, A. I. C. W. S. C. Y. S. C. S. F. Comparing four approaches for technical debt identification. 1-24.

[111] Zazworka, N. Shaw, M. S. F. S. C. Investigating the impact of design debt on software quality - mtd. 2nd International Workshop on Managing Technical Debt Waikiki, Honolulu, HI, USA.

[112] Zazworka, N. Spínola, R. V. A. S. F. S. C. A case study on effectively identifying technical debt. $42-43$. 